Supporting Information for:

Small Molecule Investigation of Diels-Alder Complexes for Thermoreversible Crosslinking in Polymeric Applications

Jarrett R. Rowlett ${ }^{1}$, Peter Deglmann ${ }^{2}$, Johannes Sprafke ${ }^{2}$, Nabarun Roy ${ }^{3}$, Rolf Mülhaupt ${ }^{4}$, Bernd Bruchmann ${ }^{1,2}$

1 Joint Research Network on Advanced Materials and Systems (JONAS), Freiburg Materials Research Center, University of Freiburg, Stefan-Meier-Str. 21, D-79104 Freiburg, Germany

2 Advanced Materials and Systems Research, BASF SE, Carl-Bosch-Strasse 38, D-67056 Ludwigshafen, Germany

${ }^{3}$ BASF Polyurethanes GmbH, Elastogranstr 60, D-49448 Lemfoerde, Germany

${ }^{4}$ Institute for Macromolecular Chemistry, University of Freiburg, Stefan-Meier-Str. 31, D-79104 Freiburg, Germany

*Jarrett.rowlett@gmail.com

Table of Contents:

1. ${ }^{1} \mathrm{H}$ NMR, ${ }^{13} \mathrm{C}$ NMR, GC/HRMS - Compound I S2

2. ${ }^{1} \mathrm{H}$ NMR, ${ }^{13} \mathrm{C}$ NMR, GC/HRMS - Compound II $\quad$ S5

3. ${ }^{1} \mathrm{H}$ NMR, ${ }^{13} \mathrm{C}$ NMR, GC/HRMS - Compound III $\quad$ S8

4. ${ }^{1} \mathrm{H}$ NMR, ${ }^{13} \mathrm{C}$ NMR, GC/HRMS - Compound IV S11

5. ${ }^{1}$ H NMR, ${ }^{13}$ C NMR, GC/HRMS - Compound V S14

6. ${ }^{1} \mathrm{H}$ NMR, ${ }^{13} \mathrm{C}$ NMR, GC/HRMS - Compound VI S17

7. ${ }^{1} \mathrm{H}$ NMR, ${ }^{13} \mathrm{C}$ NMR, GC/HRMS - Compound VII S20

8. ${ }^{1} \mathrm{H}$ NMR, ${ }^{13} \mathrm{C}$ NMR, GC/HRMS - Compound VIII S23

9. Confirmation of thermal reversibility of VIII S26

10. Compound V Exchange Reaction Kinetics $\quad$ S27

11. Compound VIII Exchange Reaction Kinetics S31

12. Computational Results Calculation Information $\quad$ S35 


\section{Compound I}
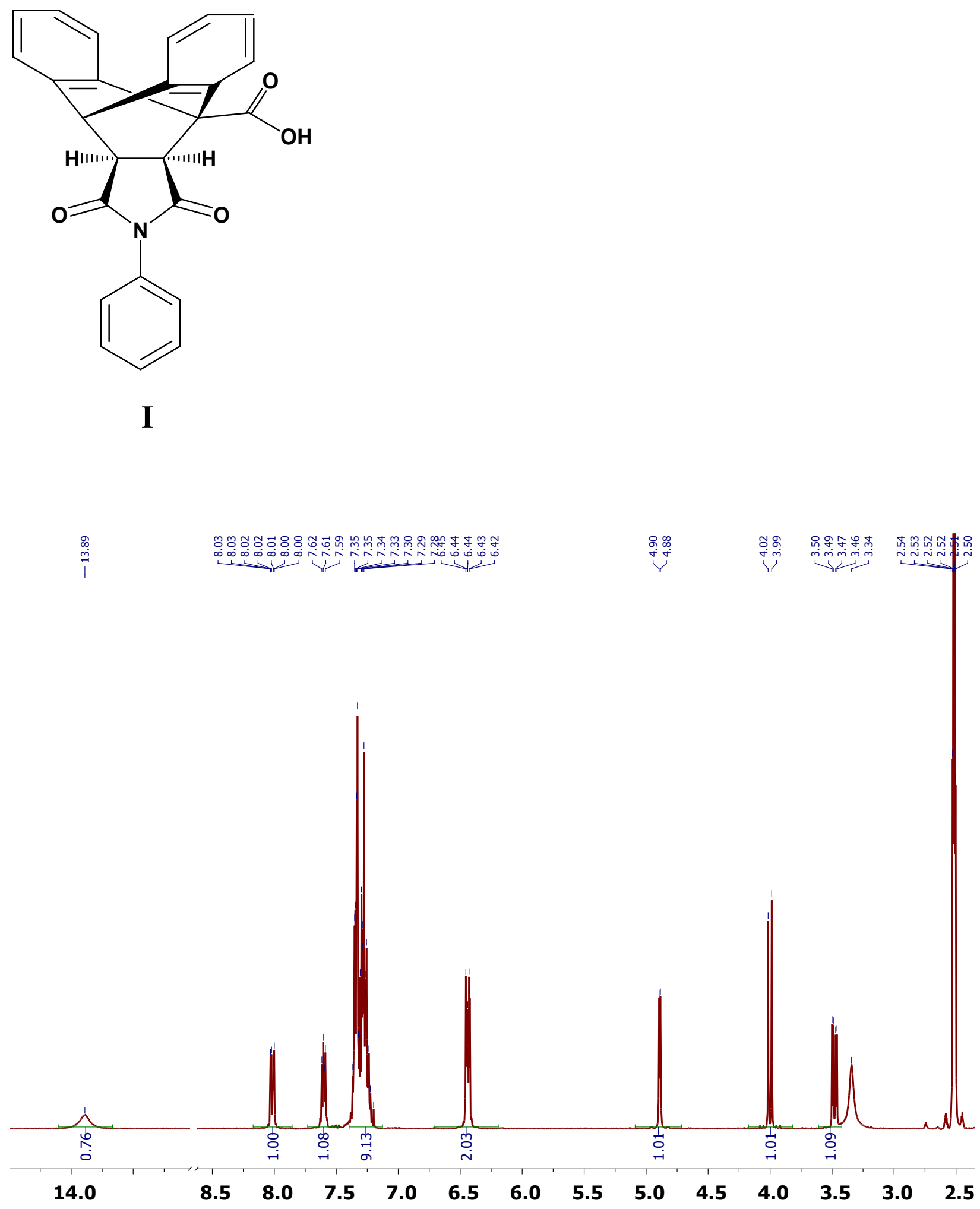

Figure S1: ${ }^{1} \mathrm{H}\left(500 \mathrm{MHz}, \mathrm{DMSO}-d_{6}\right) \mathrm{NMR}$ spectrum of Compound I 


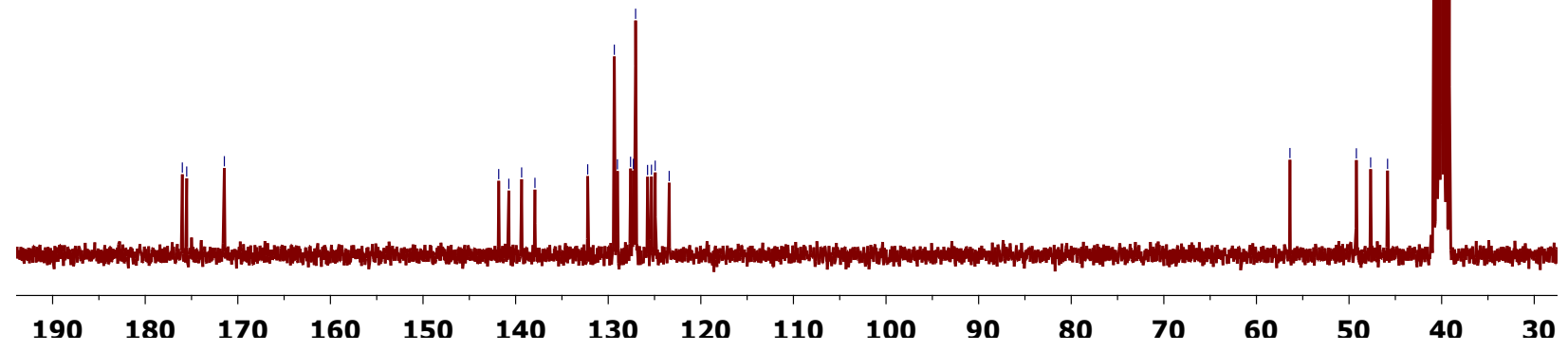

Figure S2: ${ }^{13} \mathrm{C}\left\{{ }^{1} \mathrm{H}\right\}\left(125 \mathrm{MHz}, \mathrm{DMSO}-d_{6}\right)$ NMR spectrum of Compound I 


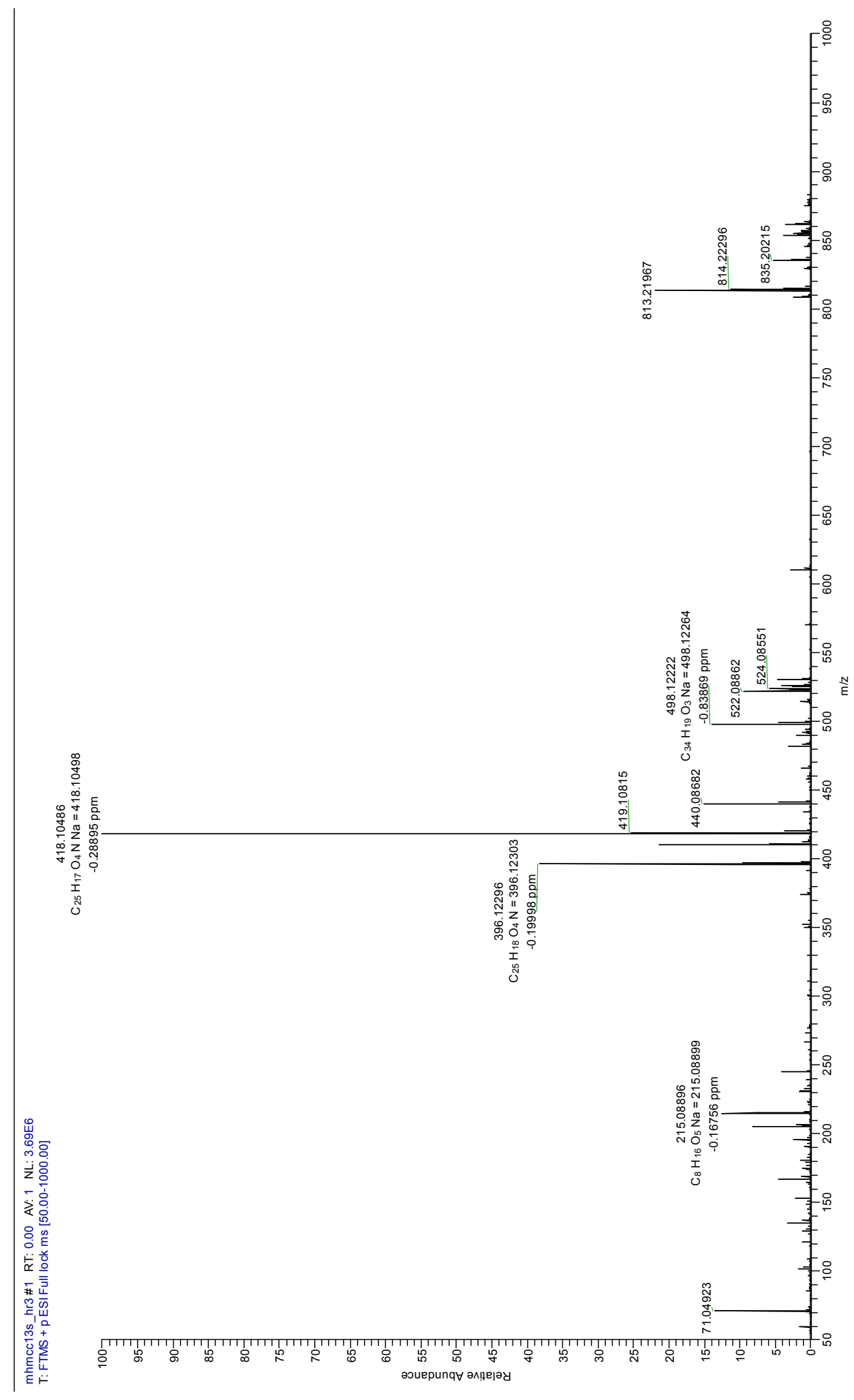




\section{Compound II}

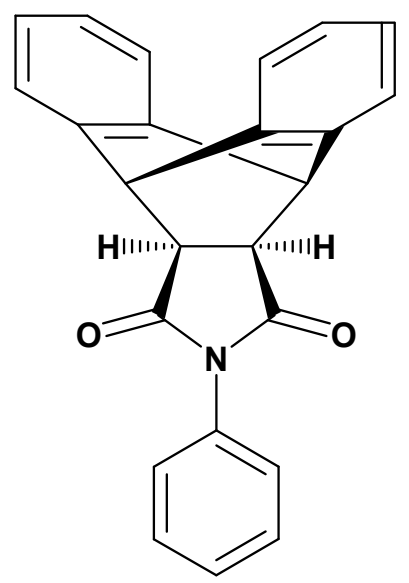

II
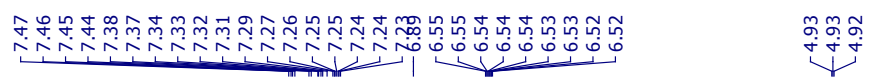

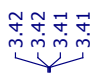
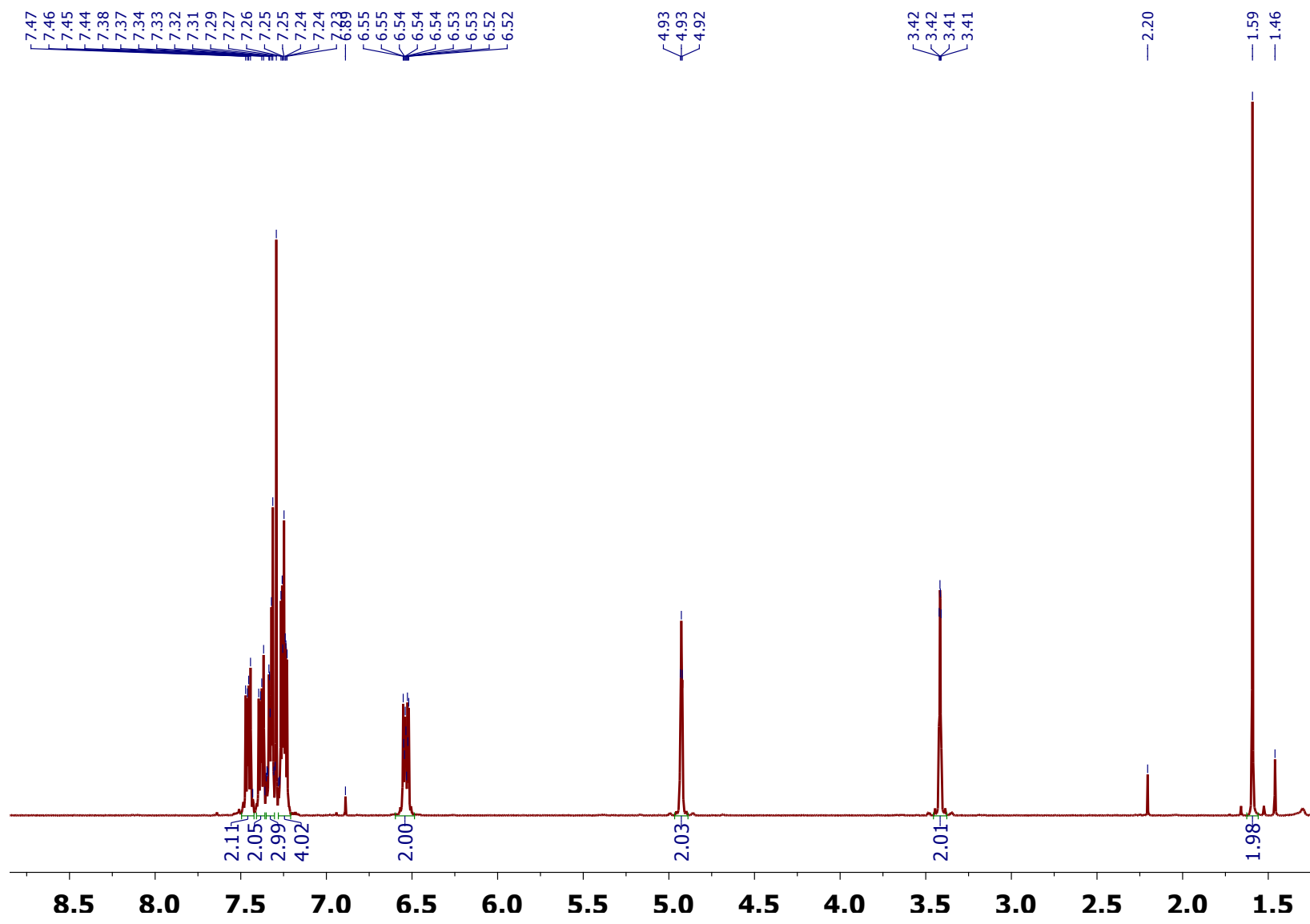

Figure S3: ${ }^{1} \mathrm{H}\left(500 \mathrm{MHz}, \mathrm{CDCl}_{3}\right) \mathrm{NMR}$ spectrum of Compound II 

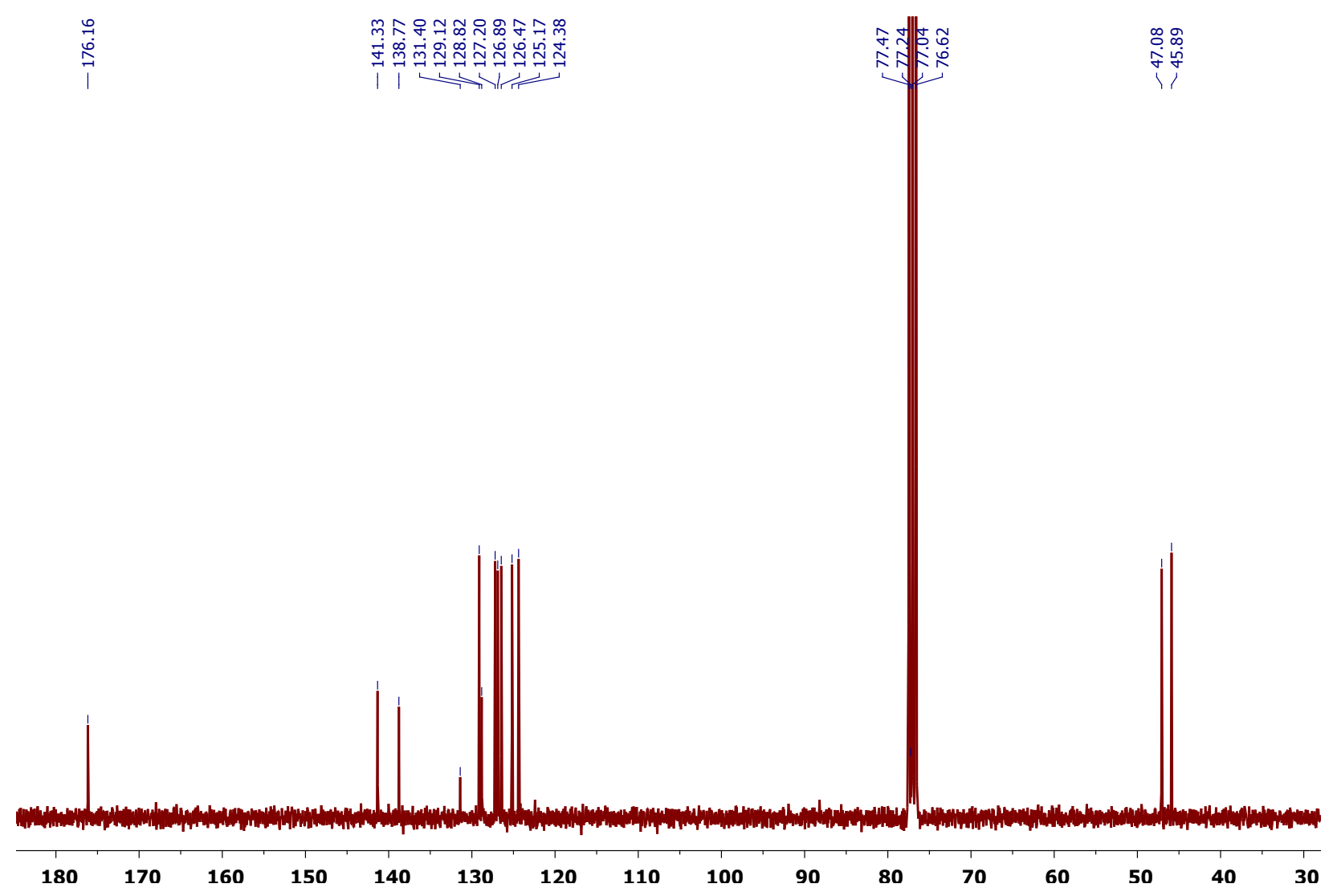

Figure S4: ${ }^{13} \mathrm{C}\left\{{ }^{1} \mathrm{H}\right\}\left(125 \mathrm{MHz}, \mathrm{CDCl}_{3}\right) \mathrm{NMR}$ spectrum of Compound II 


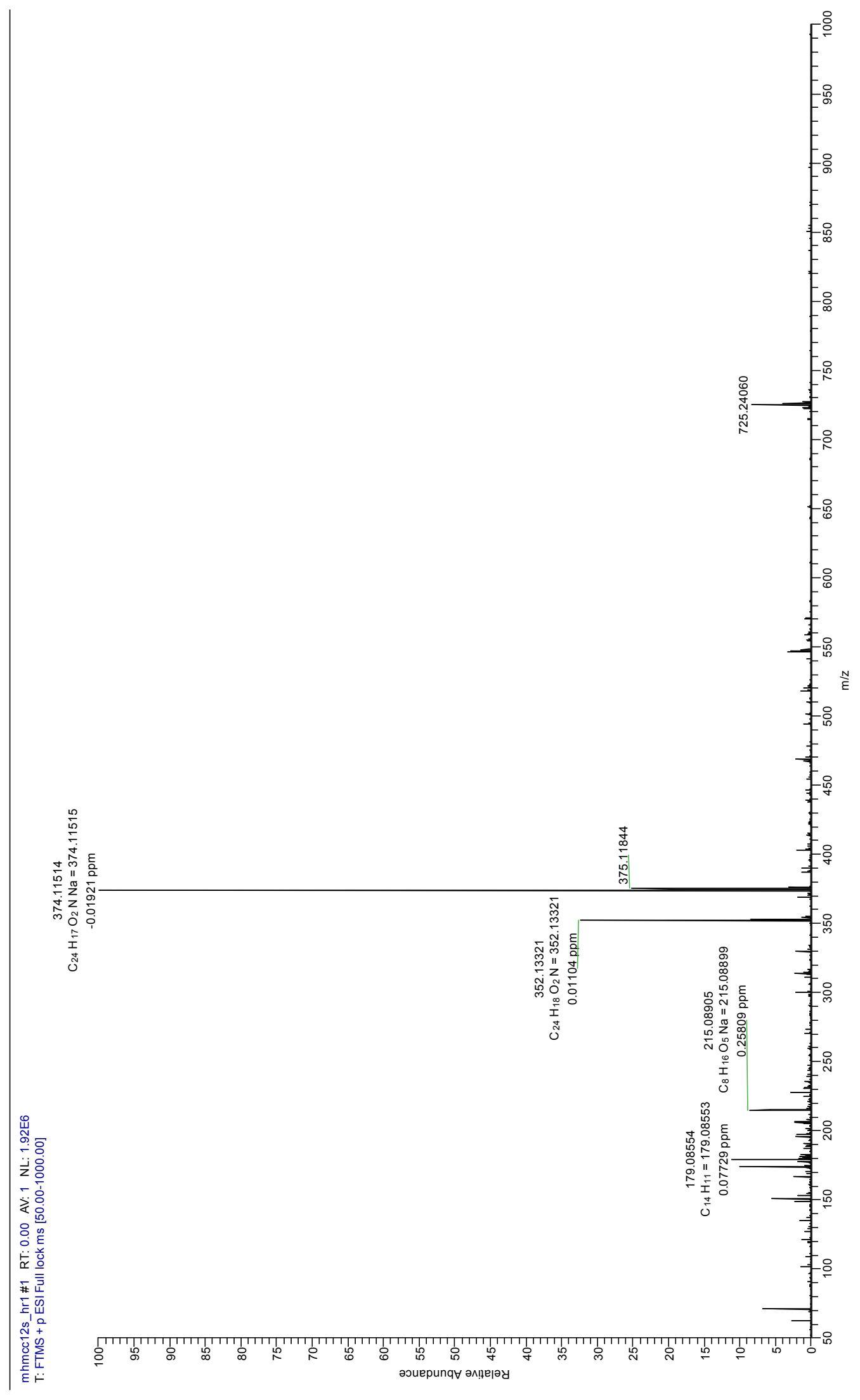




\section{Compound III}

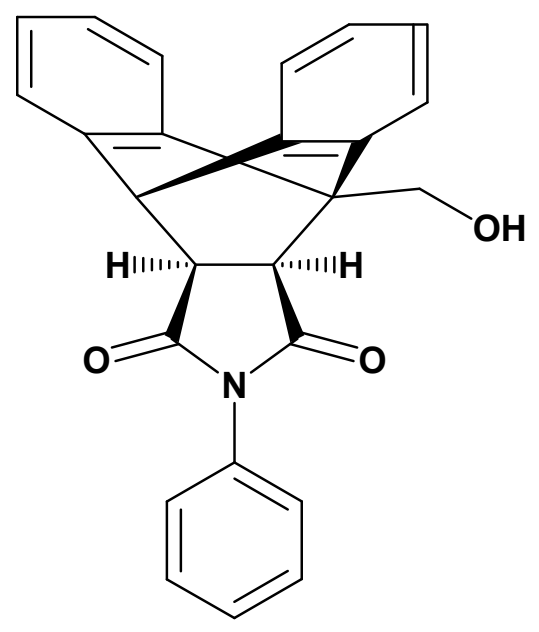

\section{III}
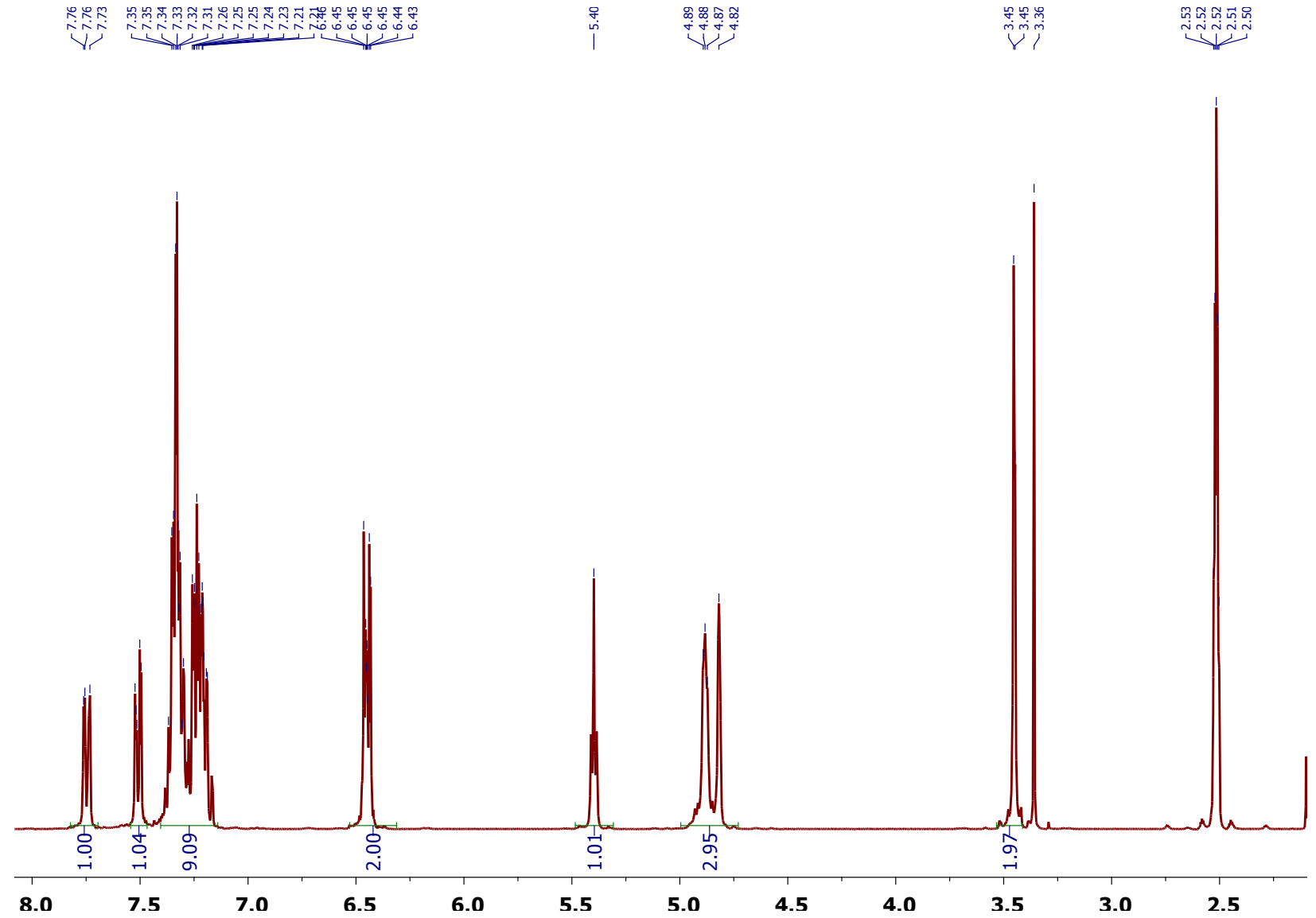

Figure S5: ${ }^{1} \mathrm{H}\left(500 \mathrm{MHz}, \mathrm{DMSO}-d_{6}\right)$ NMR spectrum of Compound III 


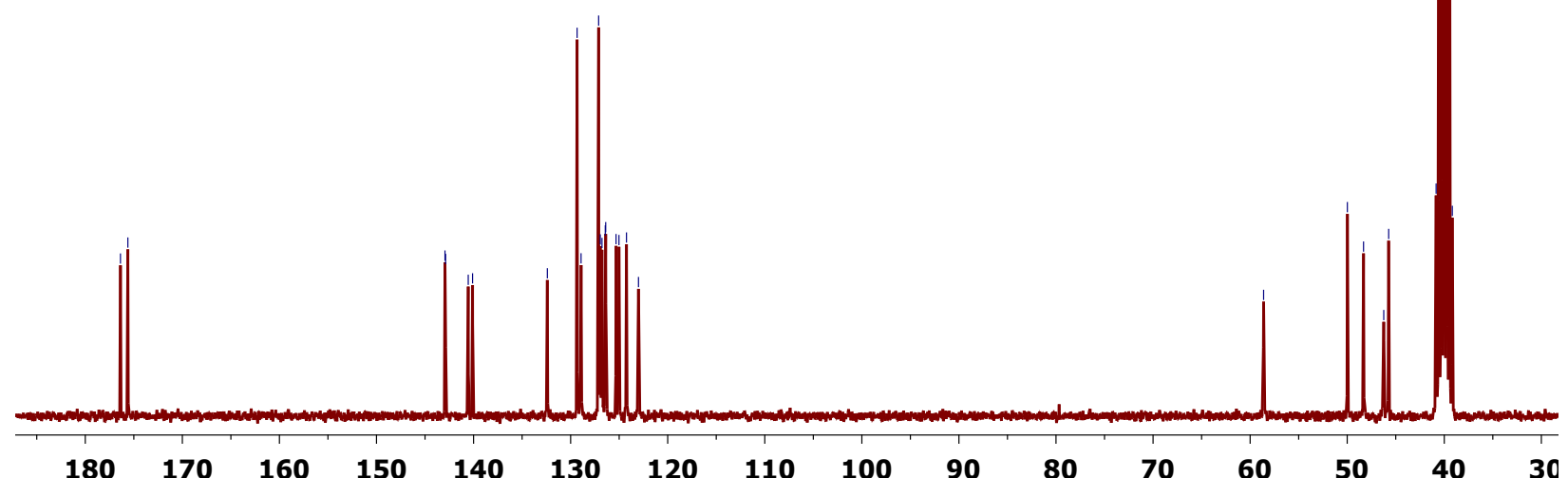

Figure S6: ${ }^{13} \mathrm{C}\left\{{ }^{1} \mathrm{H}\right\}\left(125 \mathrm{MHz}, \mathrm{DMSO}-d_{6}\right)$ NMR spectrum of Compound III 


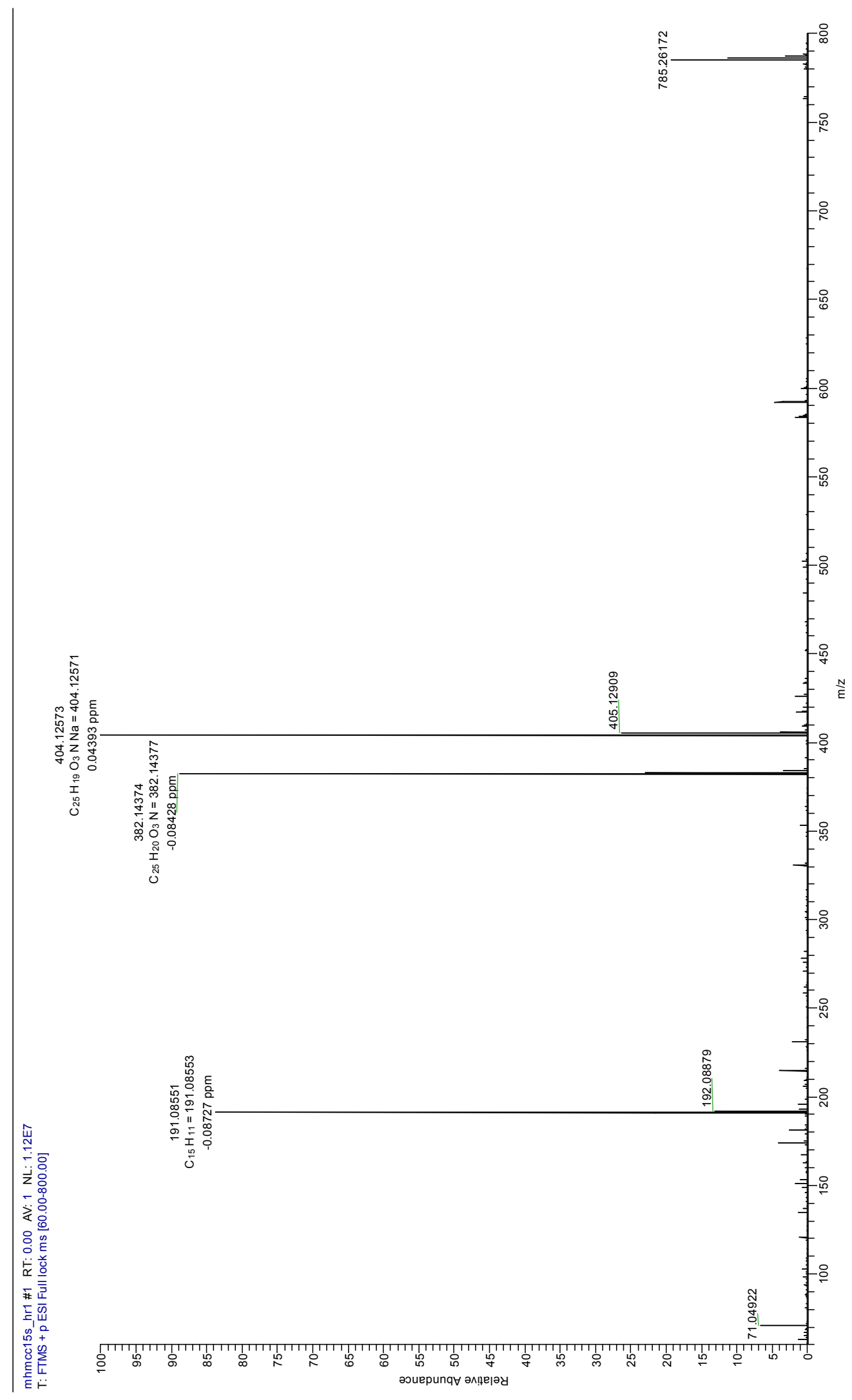




\section{Compound IV}

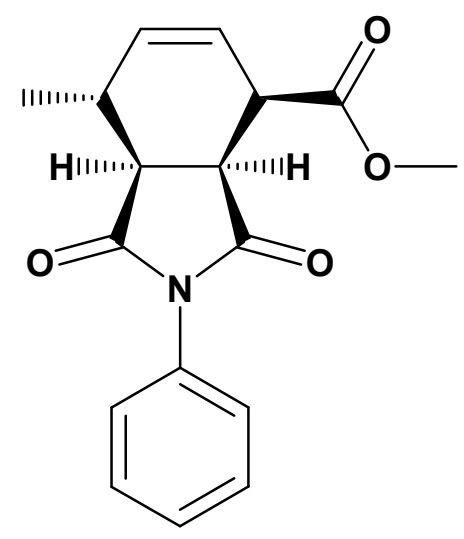

IV
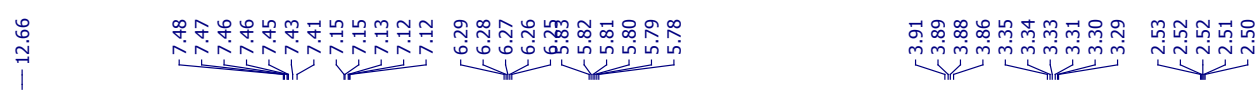

लं

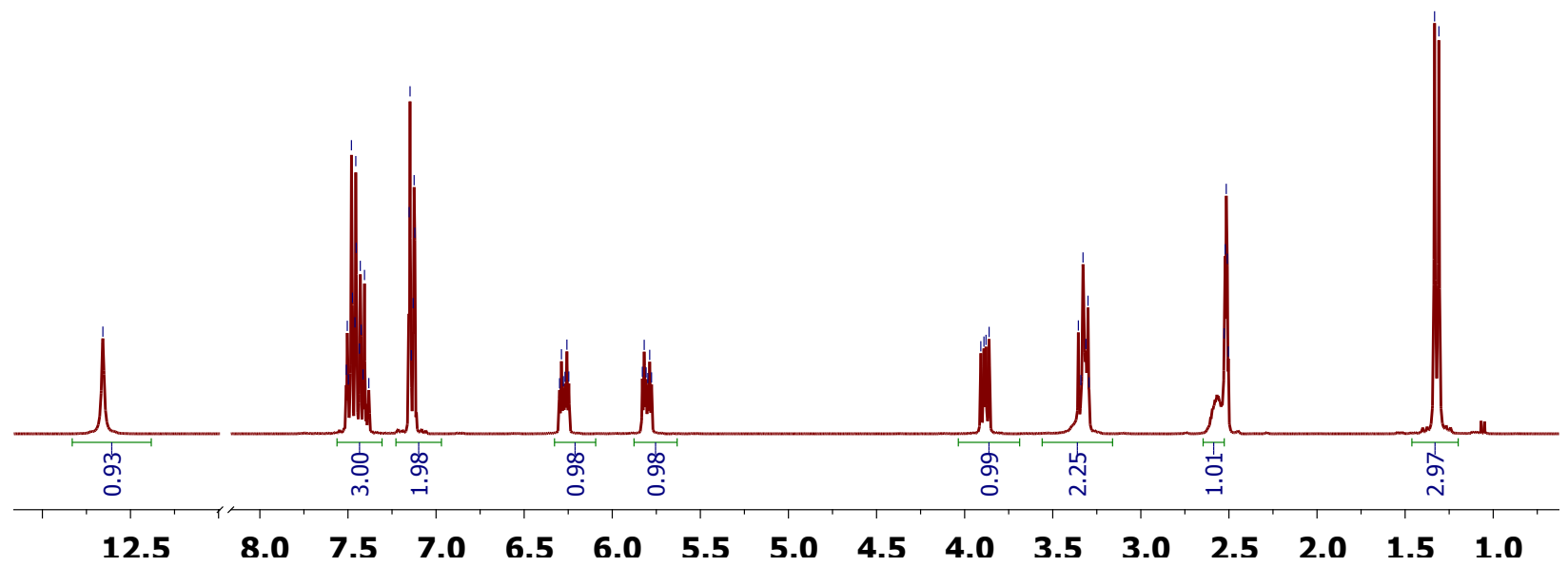

Figure S7: ${ }^{1} \mathrm{H}\left(500 \mathrm{MHz}, \mathrm{DMSO}-d_{6}\right) \mathrm{NMR}$ spectrum of Compound IV 


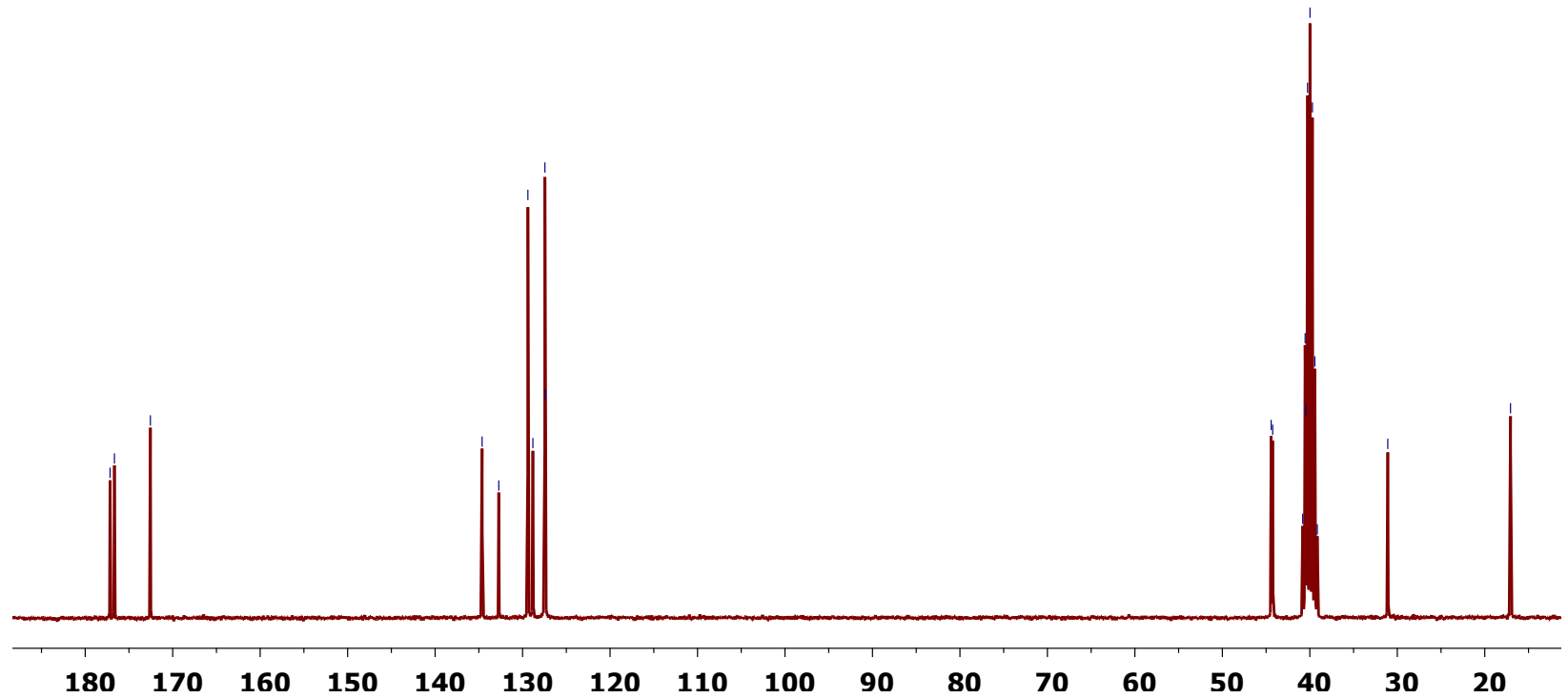

Figure S8: ${ }^{13} \mathrm{C}\left\{{ }^{1} \mathrm{H}\right\}\left(125 \mathrm{MHz}, \mathrm{DMSO}-d_{6}\right)$ NMR spectrum of Compound IV 


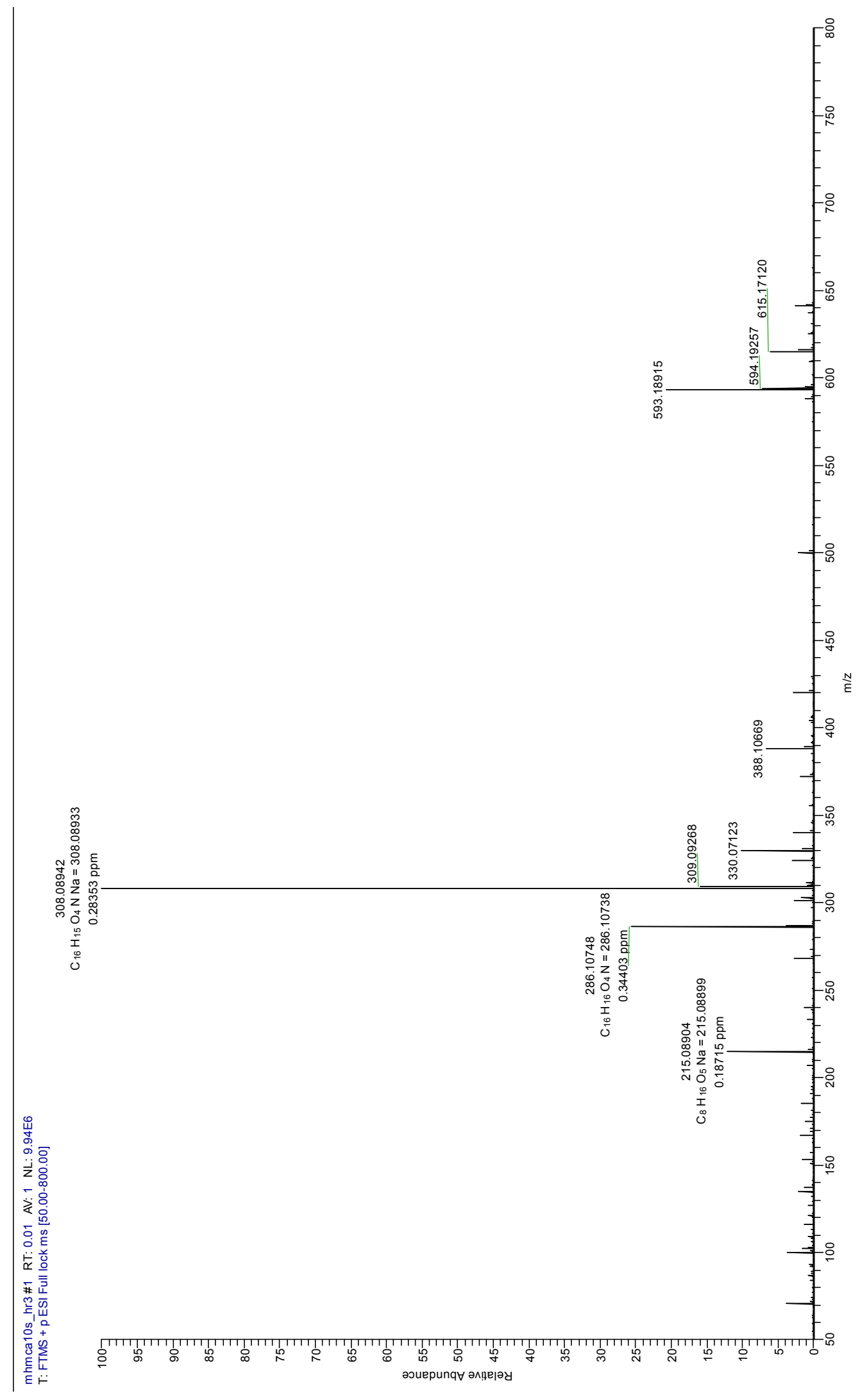




\section{Compound V}
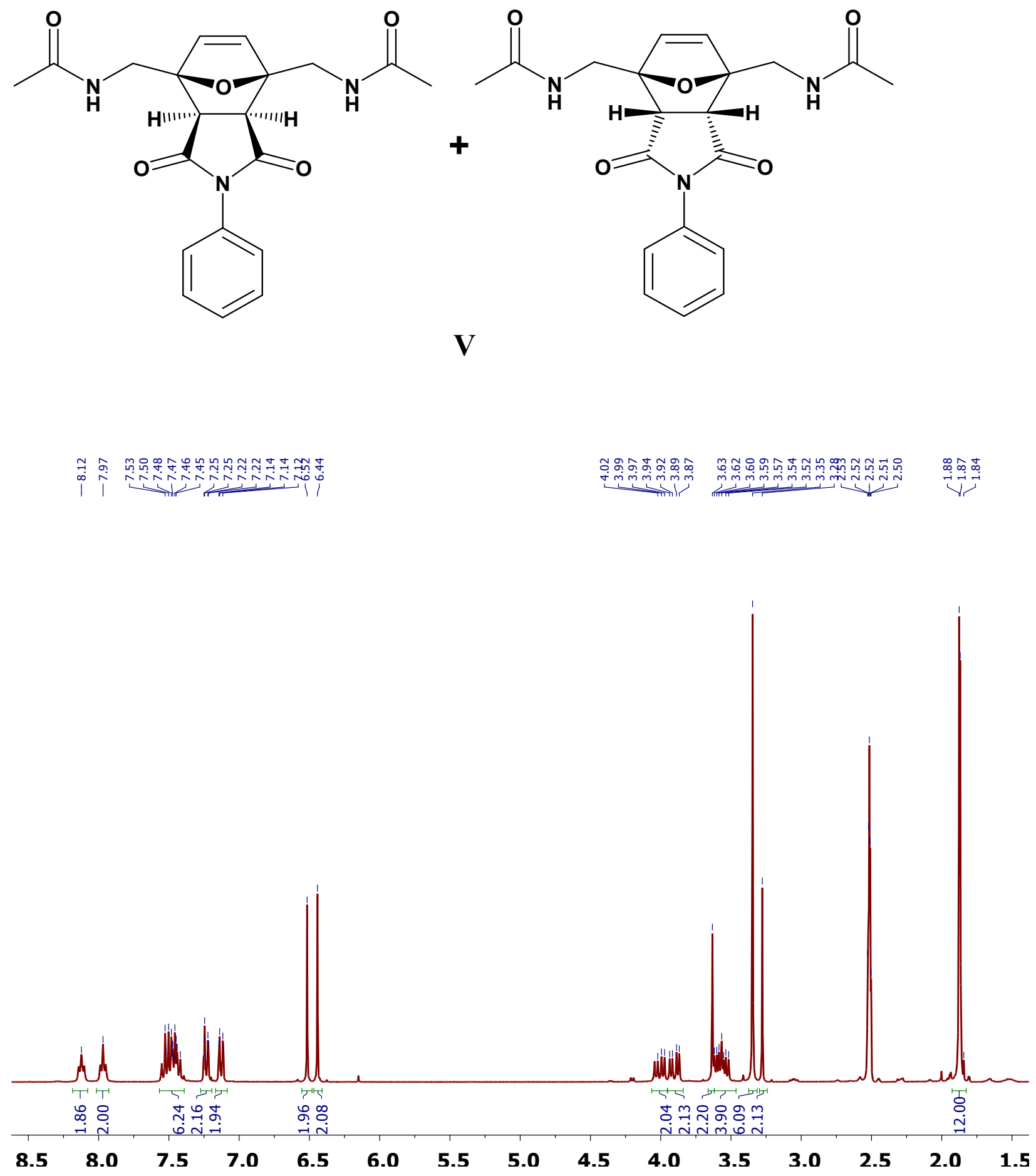

Figure S9: ${ }^{1} \mathrm{H}\left(500 \mathrm{MHz}, \mathrm{DMSO}-d_{6}\right) \mathrm{NMR}$ spectrum of Compound V (both isomers) 


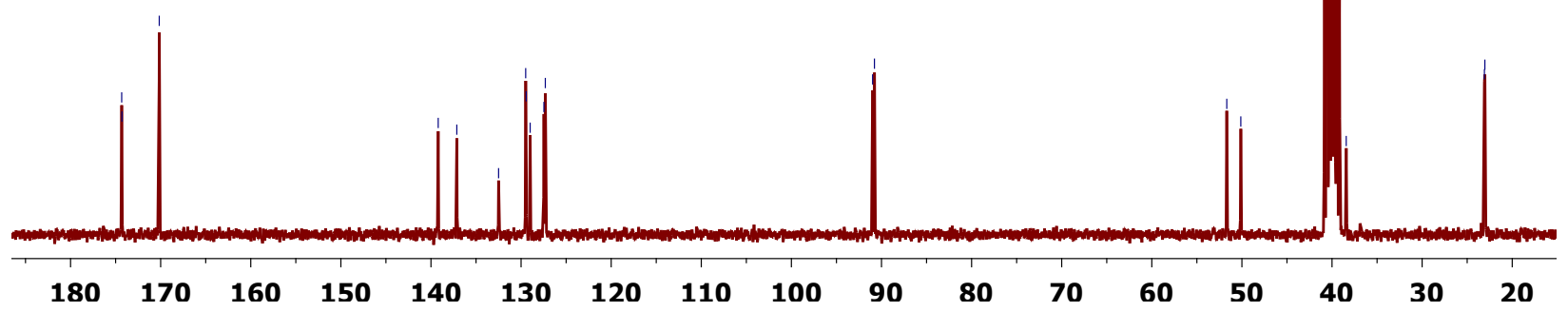

Figure S10: ${ }^{13} \mathrm{C}\left\{{ }^{1} \mathrm{H}\right\}\left(125 \mathrm{MHz}, \mathrm{DMSO}-d_{6}\right)$ NMR spectrum of Compound V (both isomers) 


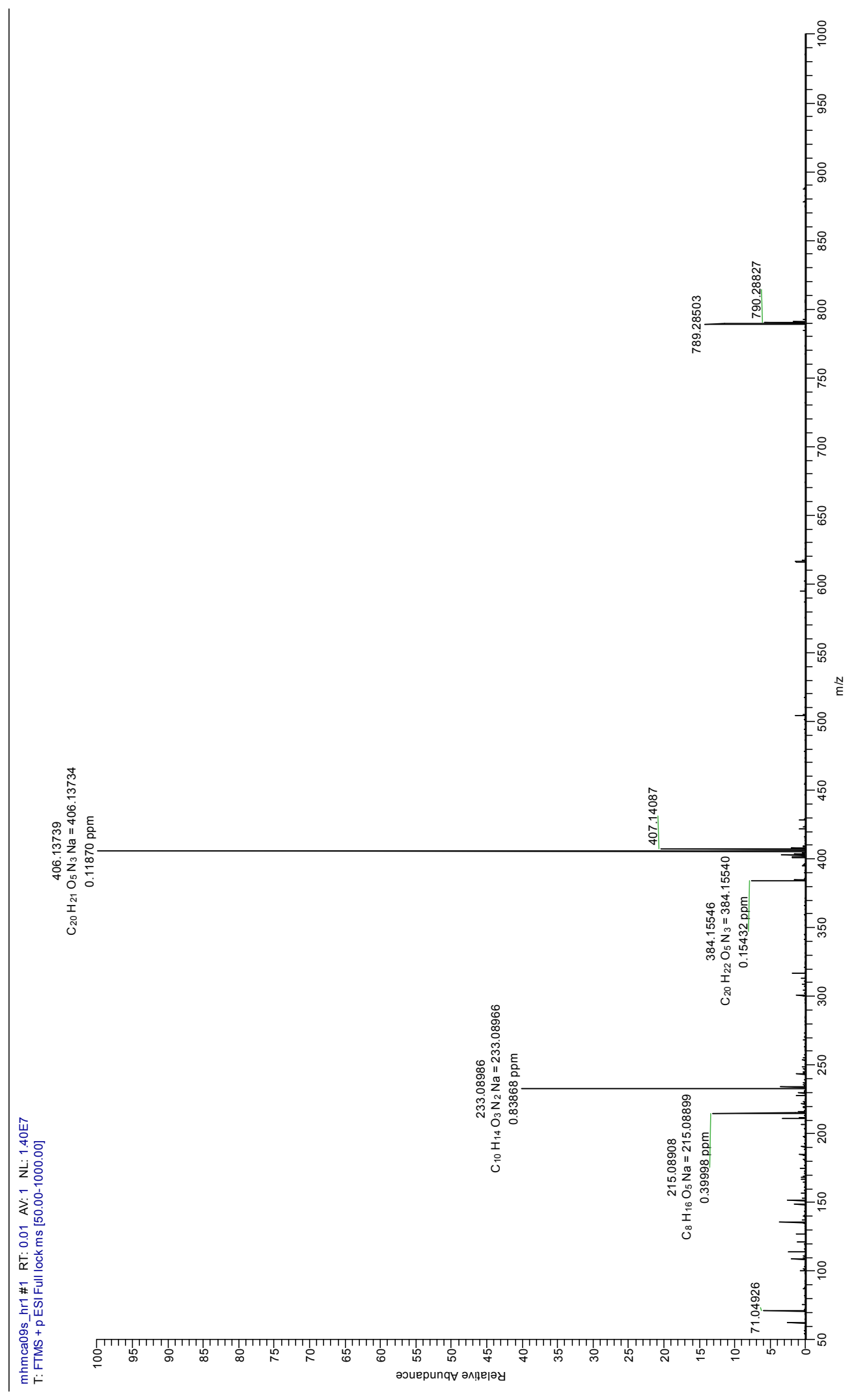




\section{Compound VI}

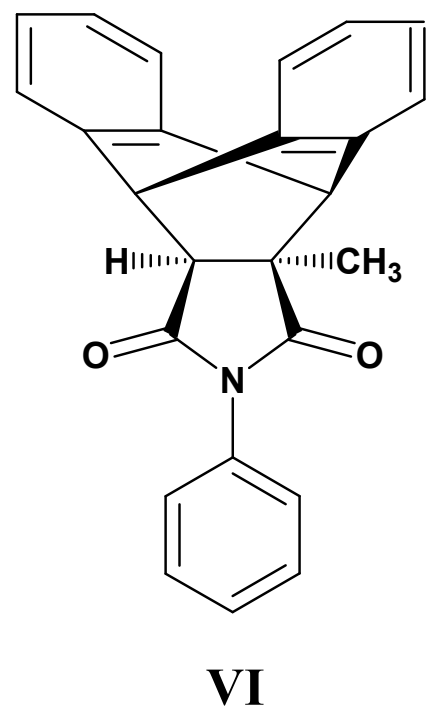

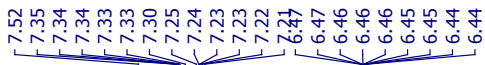

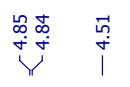

怂

$\stackrel{9}{\rightarrow}$

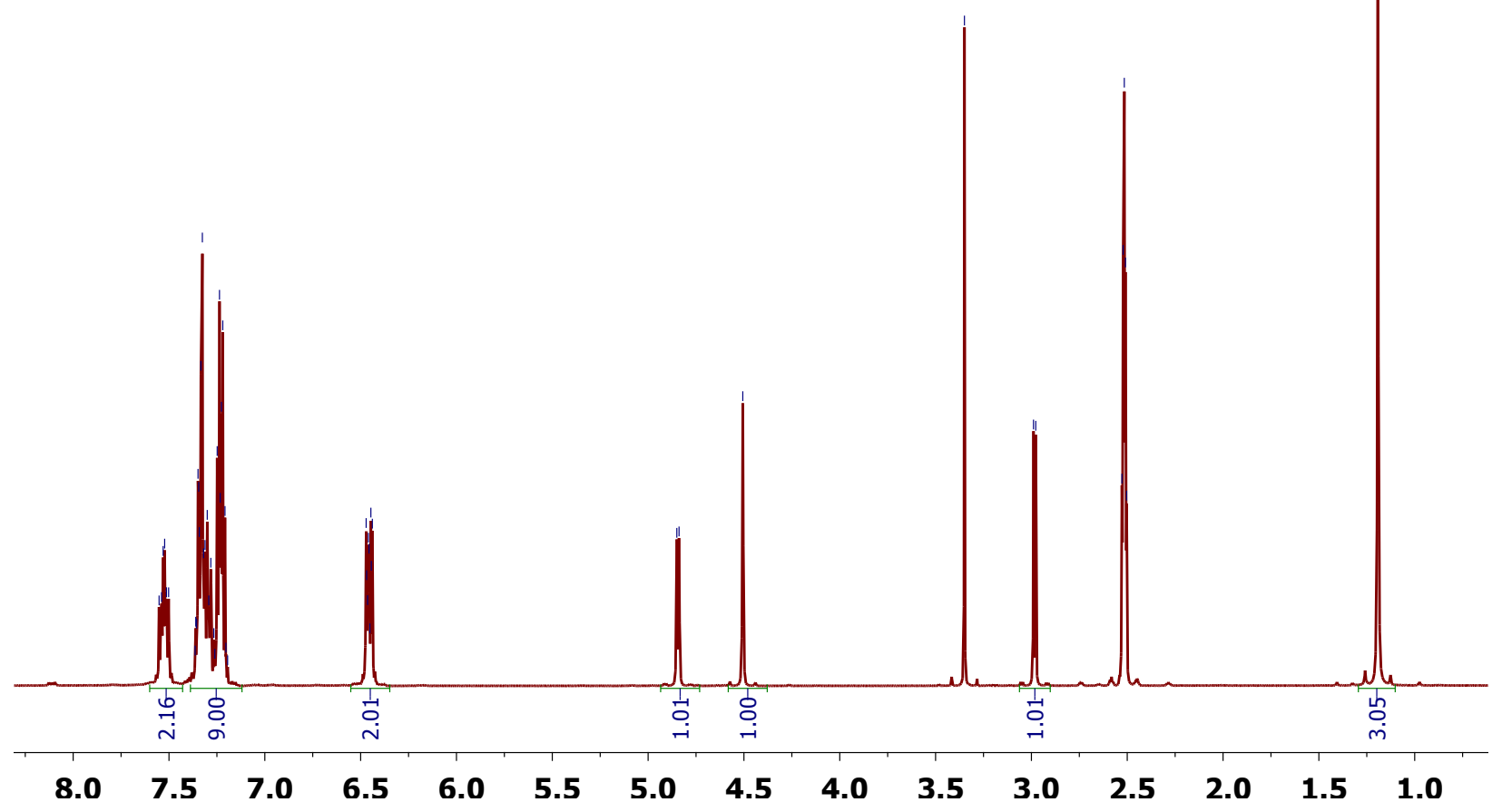

Figure S11: ${ }^{1} \mathrm{H}\left(500 \mathrm{MHz}, \mathrm{DMSO}-d_{6}\right)$ NMR spectrum of Compound VI 


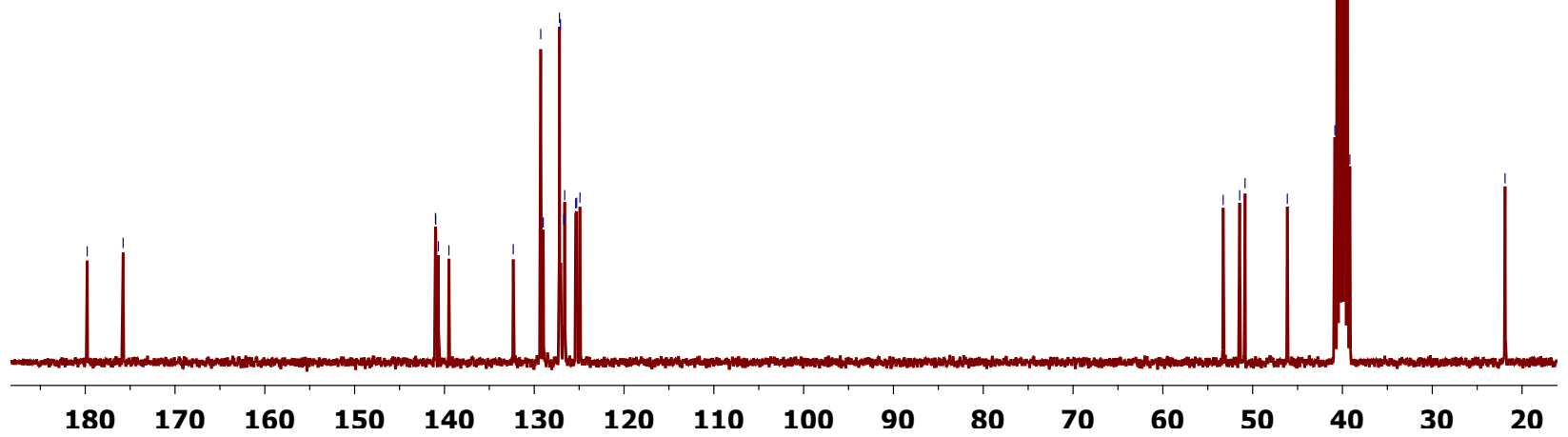

Figure S12: ${ }^{13} \mathrm{C}\left\{{ }^{1} \mathrm{H}\right\}\left(125 \mathrm{MHz}, \mathrm{DMSO}-d_{6}\right)$ NMR spectrum of Compound VI 


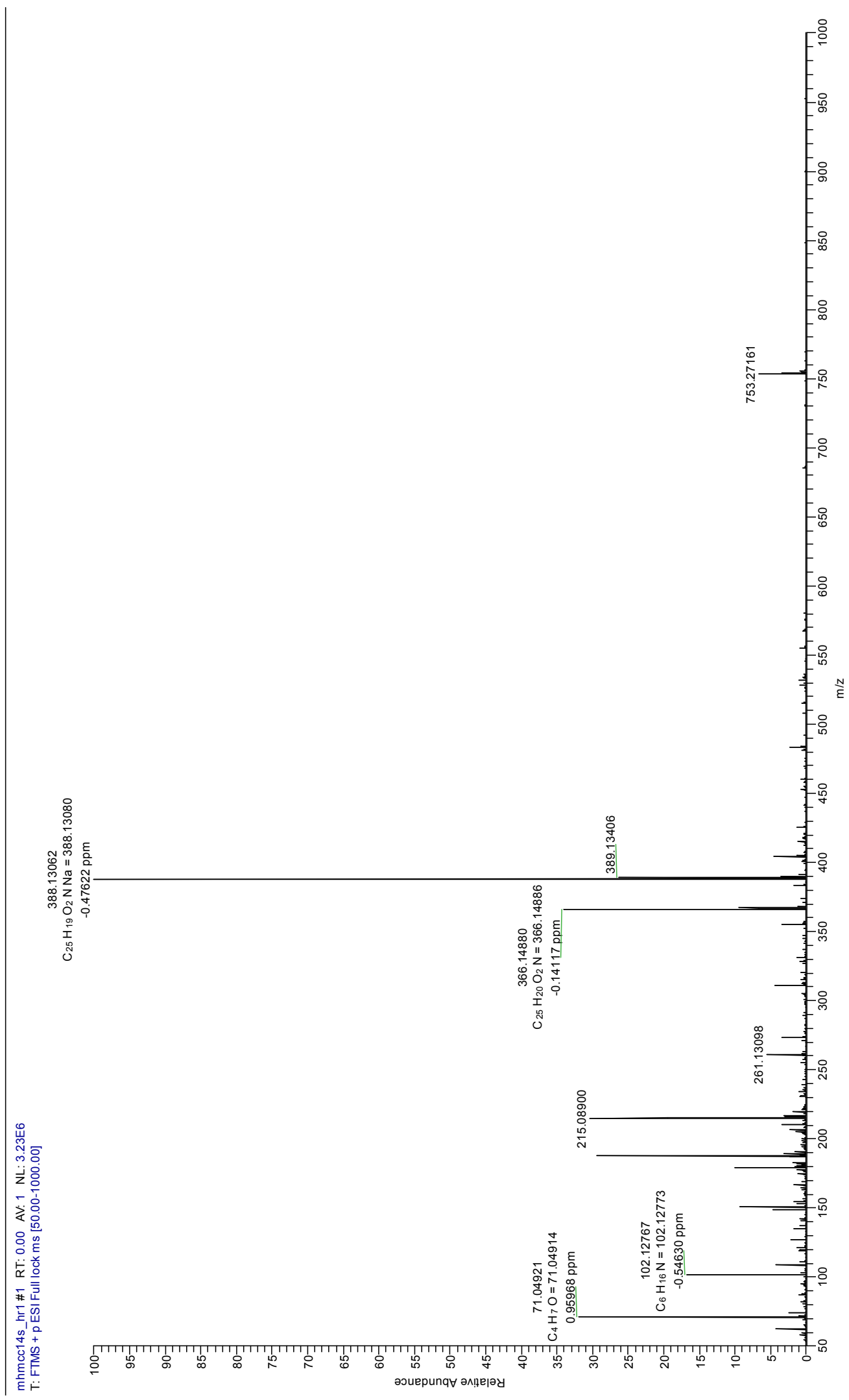




\section{Compound VII}
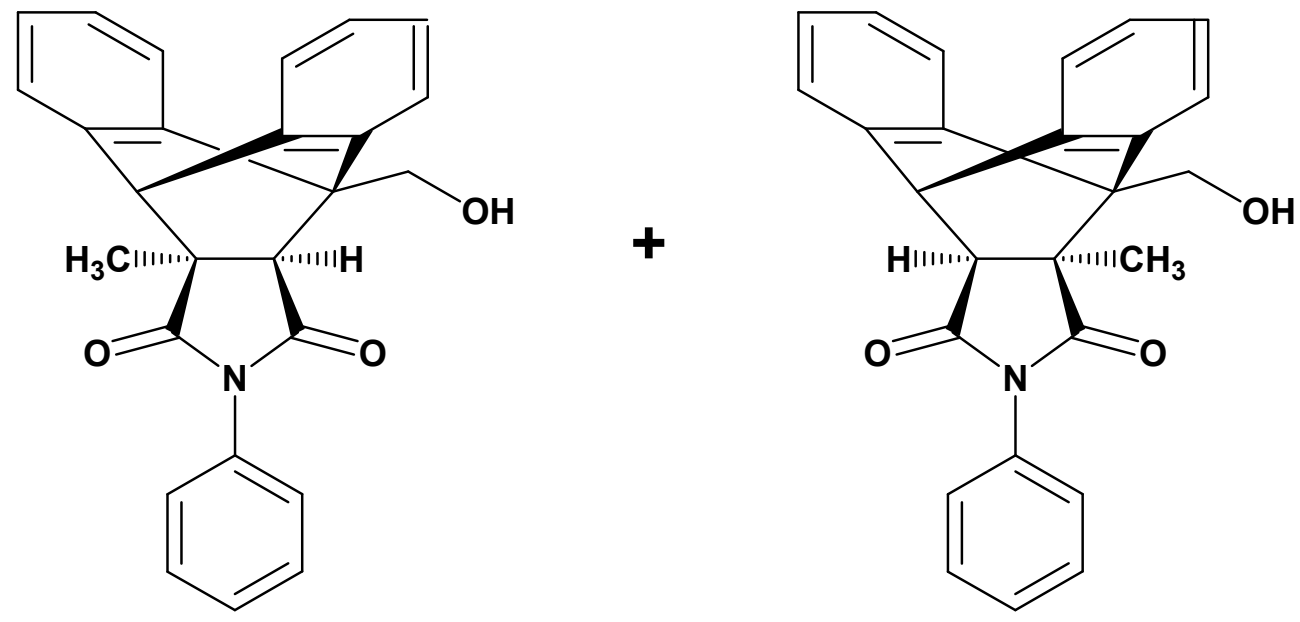

\section{VII}

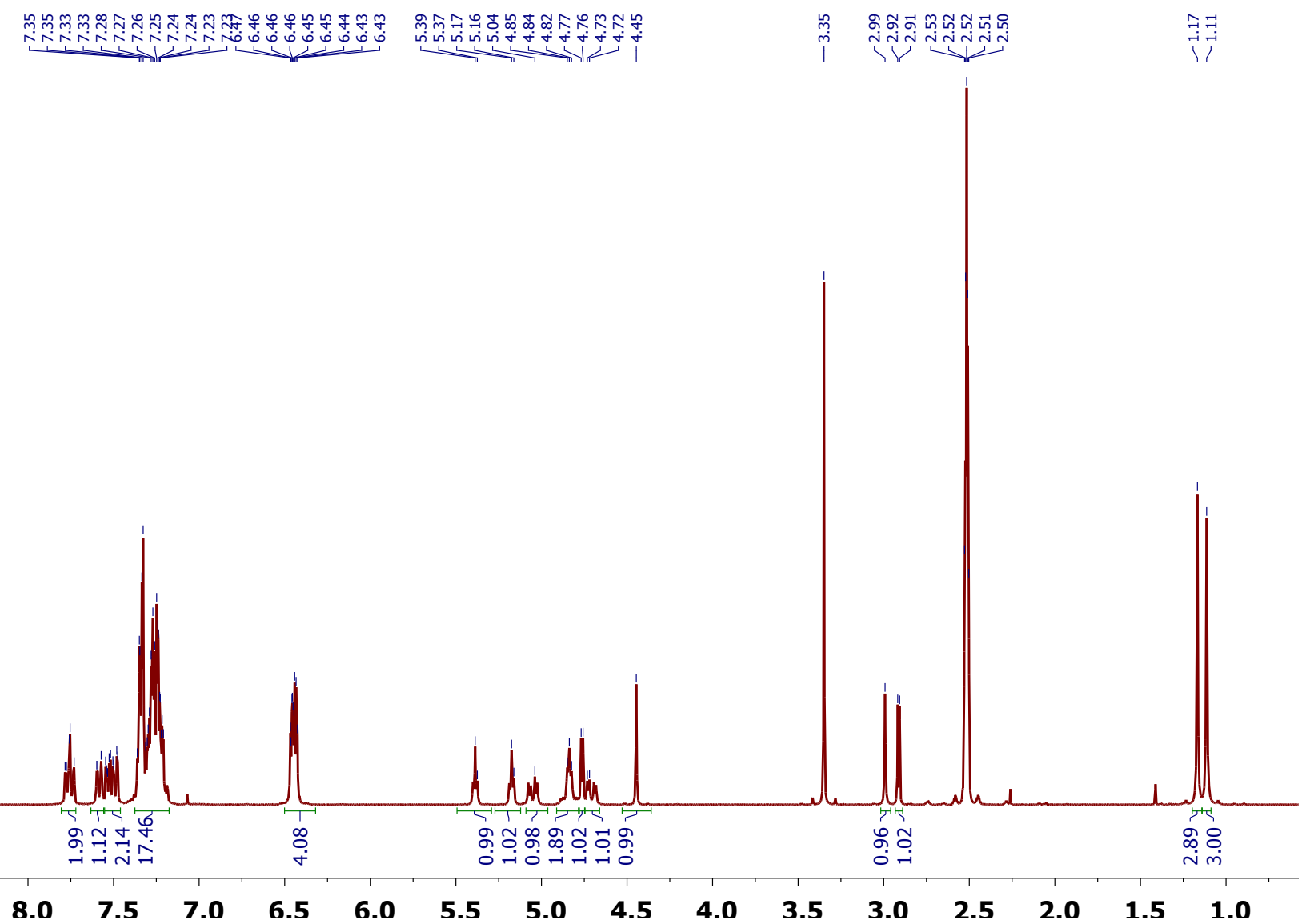

Figure S13: ${ }^{1} \mathrm{H}\left(500 \mathrm{MHz}, \mathrm{DMSO}-d_{6}\right)$ NMR spectrum of Compound VII (both isomers) 


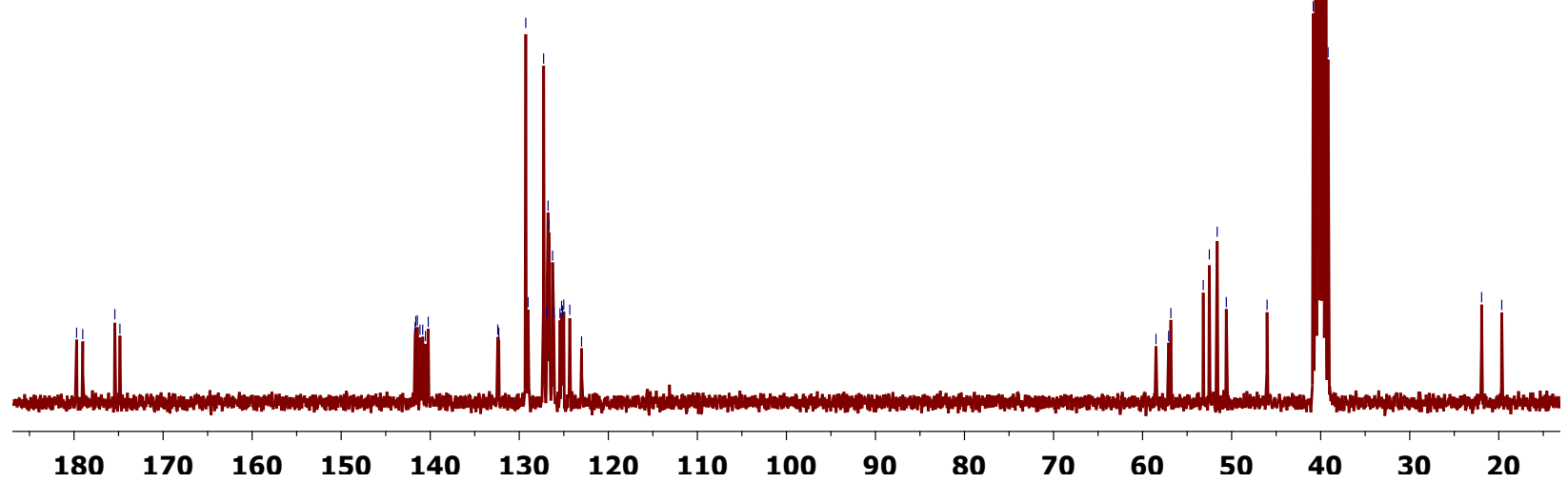

Figure S14: ${ }^{13} \mathrm{C}\left\{{ }^{1} \mathrm{H}\right\}\left(125 \mathrm{MHz}, \mathrm{DMSO}-d_{6}\right)$ NMR spectrum of Compound VII (both isomers) 


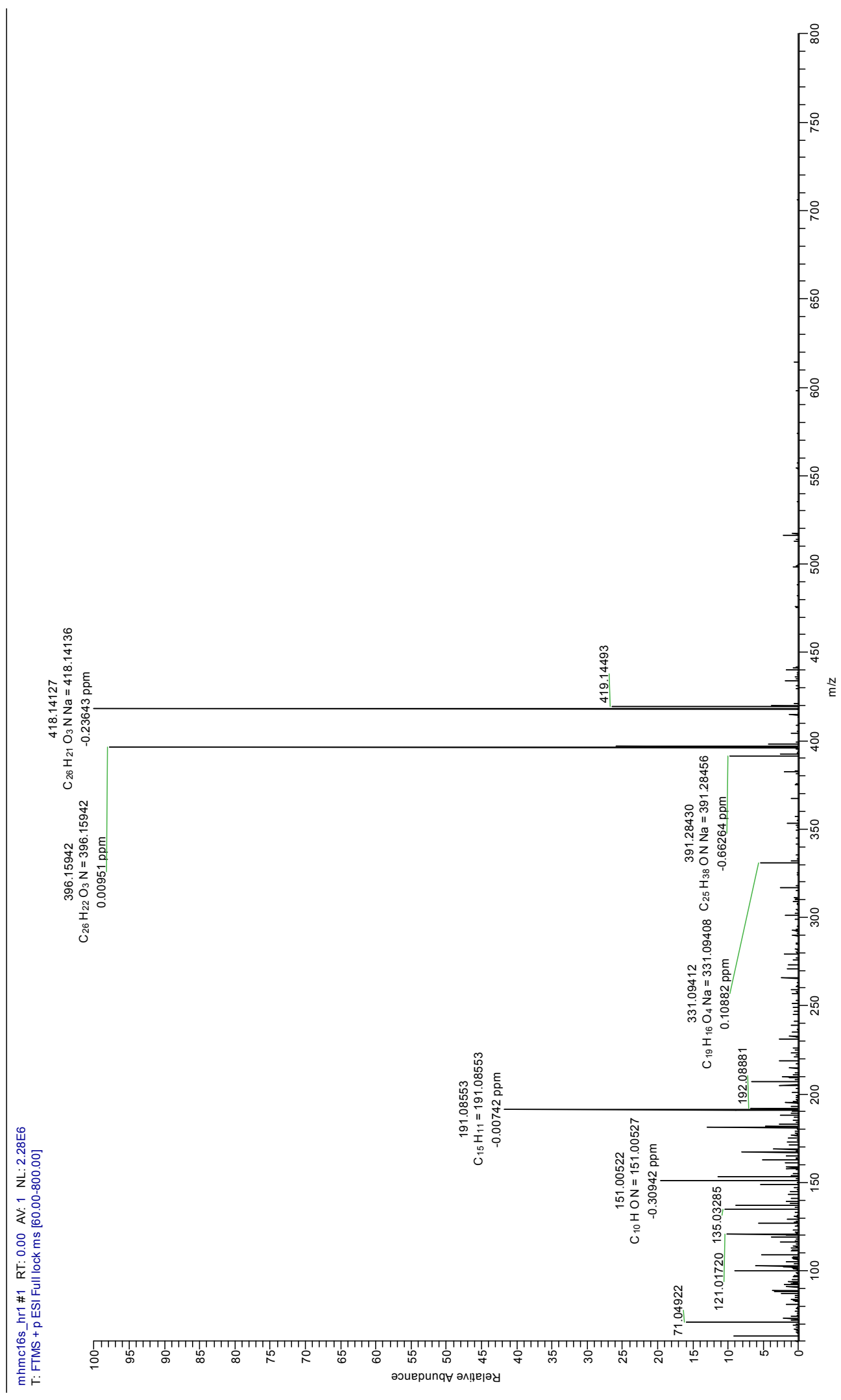




\section{Compound VIII}

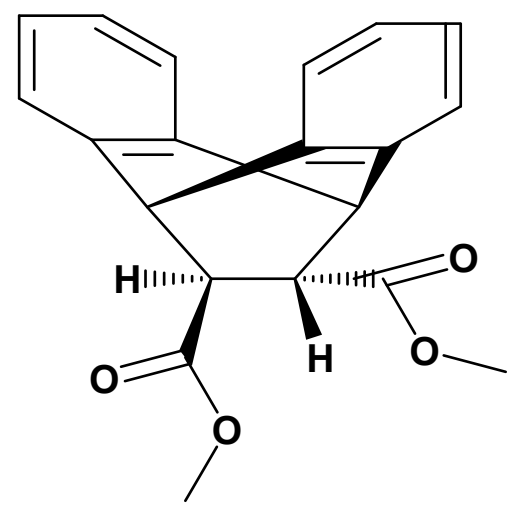

VIII

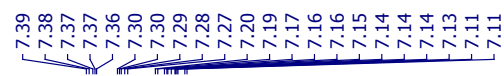
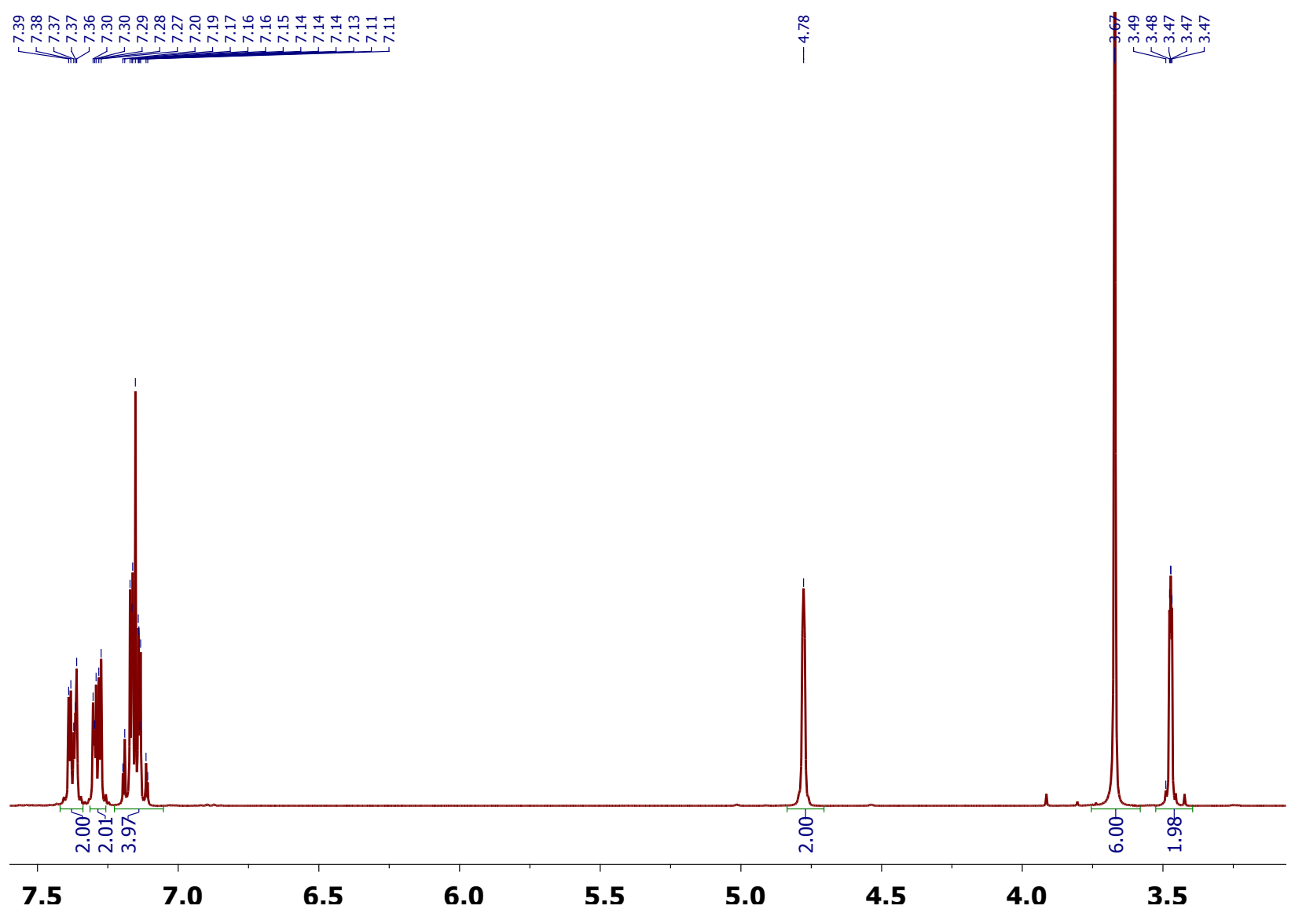

Figure S15: ${ }^{1} \mathrm{H}\left(500 \mathrm{MHz}, \mathrm{CDCl}_{3}\right)$ NMR spectrum of Compound VIII 


\begin{tabular}{|c|c|c|c|c|}
\hline 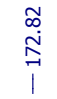 & 总令 & 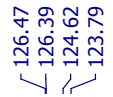 & 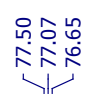 & 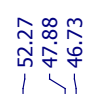 \\
\hline
\end{tabular}

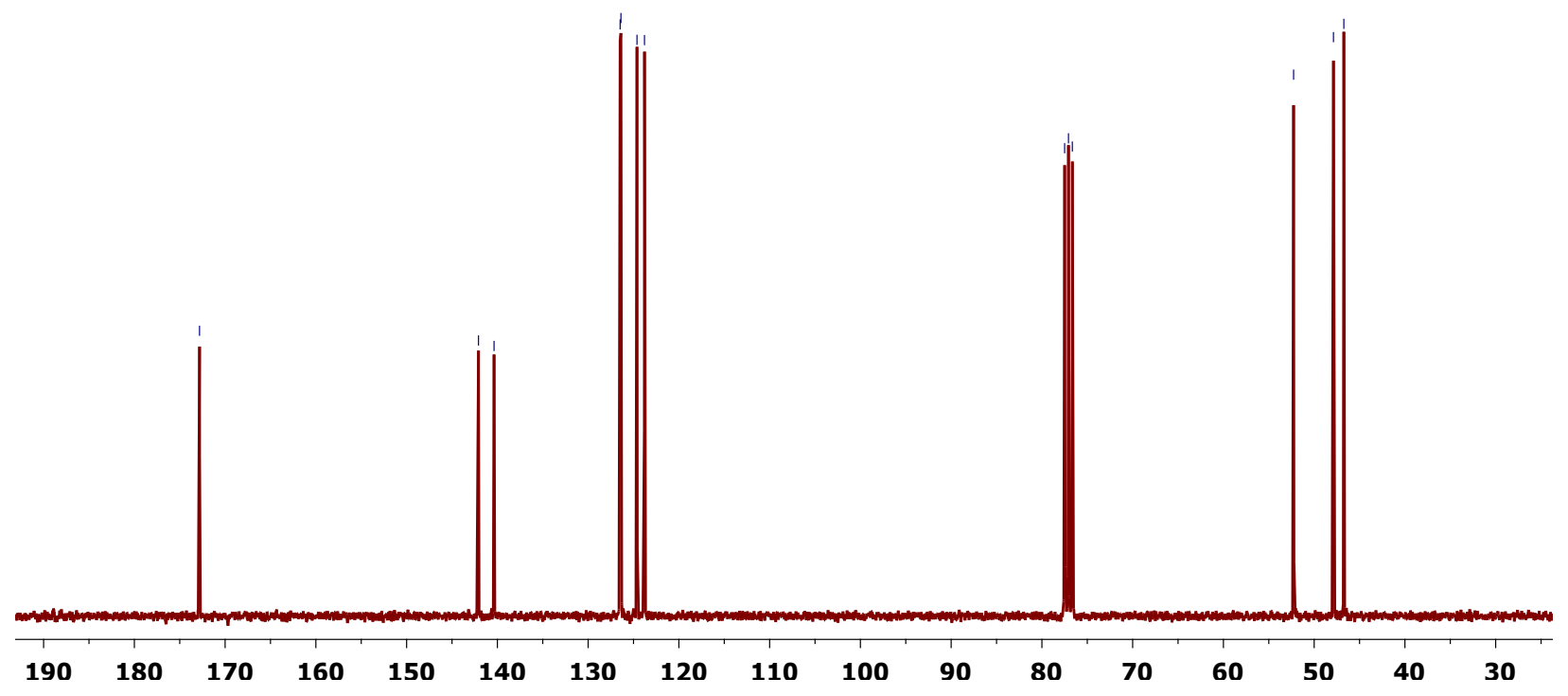

Figure S16: ${ }^{13} \mathrm{C}\left\{{ }^{1} \mathrm{H}\right\}\left(125 \mathrm{MHz}, \mathrm{DMSO}-d_{6}\right)$ NMR spectrum of Compound VIII 

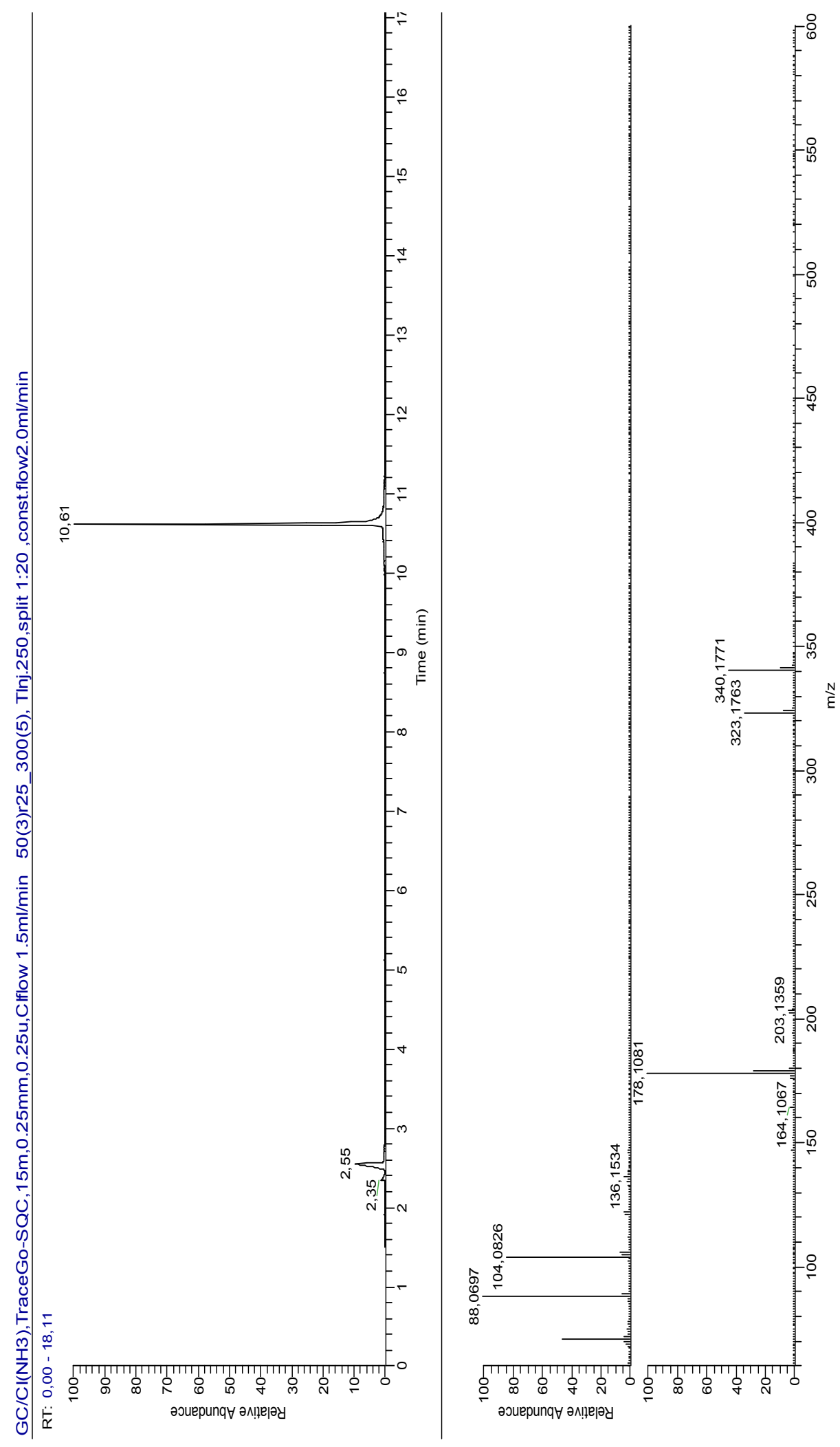


\section{Confirmation of thermal reversibility of VIII}

For this test the compound was heated at $225^{\circ} \mathrm{C}$ for the indicated time and then half of the mixture was quenched and the other half was allowed to slowly return to room temperature. After sitting overnight the NMRs were taken and compared. It was clear in the mixture that was quenched that there was a higher amount of free anthracene present, indicating reversible of the system.

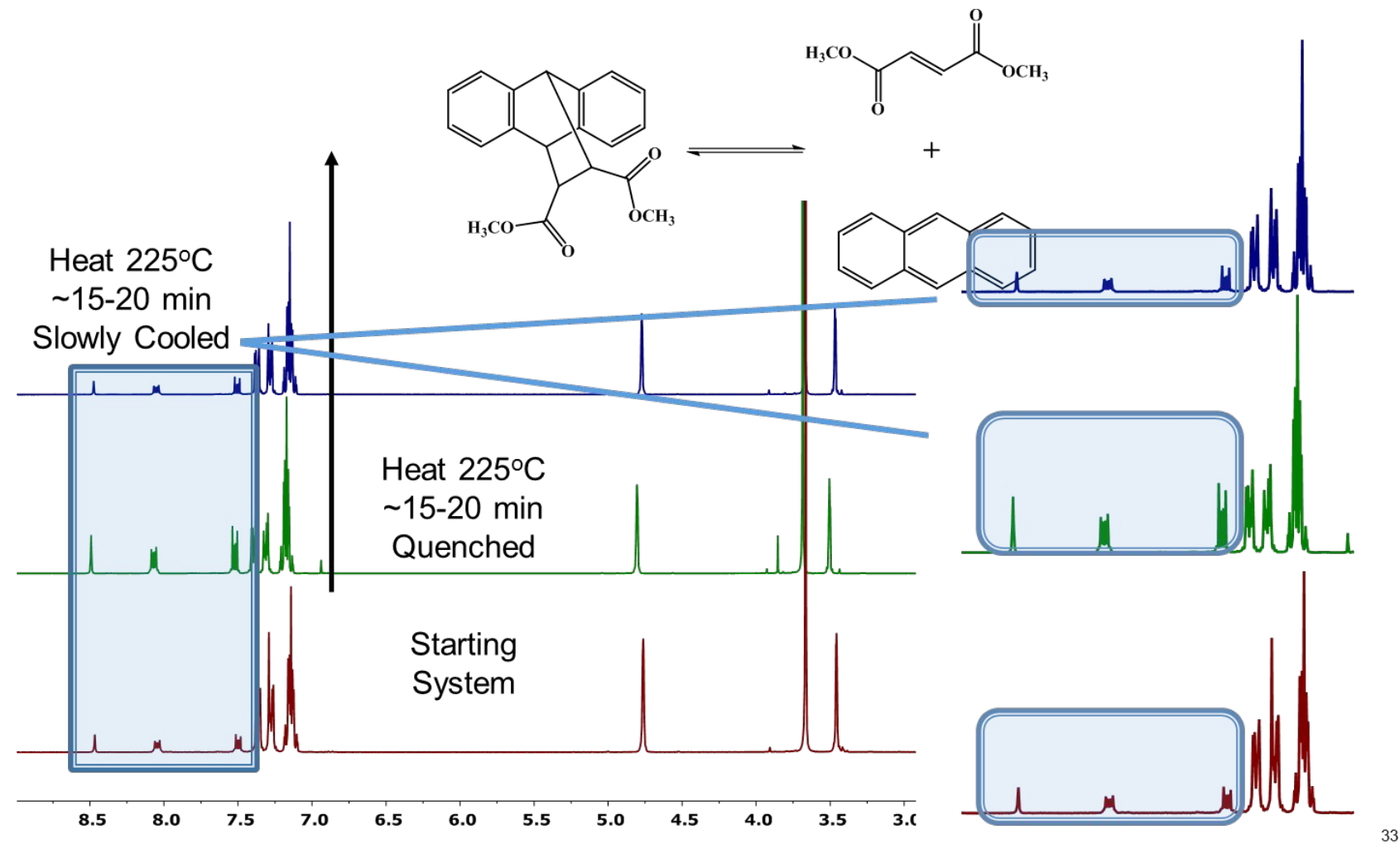


10. Compound V Exchange Reaction Kinetics

\begin{tabular}{|c|c|c|c|}
\hline Compound & Temperature & Time (min) & Exchange \% \\
\hline V & 65 & 60 & 14.5 \\
\hline V & 65 & 150 & 26.5 \\
\hline V & 65 & 330 & 42 \\
\hline $\mathrm{V}$ & 65 & 780 & 63 \\
\hline V & 65 & 1440 & 79 \\
\hline V & 65 & 2970 & 95 \\
\hline V & 85 & 60 & 39 \\
\hline V & 85 & 120 & 63 \\
\hline V & 85 & 180 & 79 \\
\hline V & 85 & 240 & 88 \\
\hline V & 85 & 300 & 92 \\
\hline V & 105 & 5 & 20 \\
\hline V & 105 & 10 & 50 \\
\hline V & 105 & 15 & 65 \\
\hline V & 105 & 25 & 80 \\
\hline V & 105 & 50 & 92 \\
\hline V & 105 & 70 & 97 \\
\hline
\end{tabular}


${ }^{1} \mathrm{H}$ NMR of in-situ exchange reaction for Compound $\mathbf{V}$ at $65^{\circ} \mathrm{C}$. Time increases from the bottom to the top of the figure. The exchange percentage of the reaction was measure by the appearance of the peak at $\sim 6.2 \mathrm{ppm}$, which belongs to the unsaturated hydrogens of Diene 5 , to the methoxy hydrogens at $\sim 1.9 \mathrm{ppm}$, which do not shift during the reverse reaction.

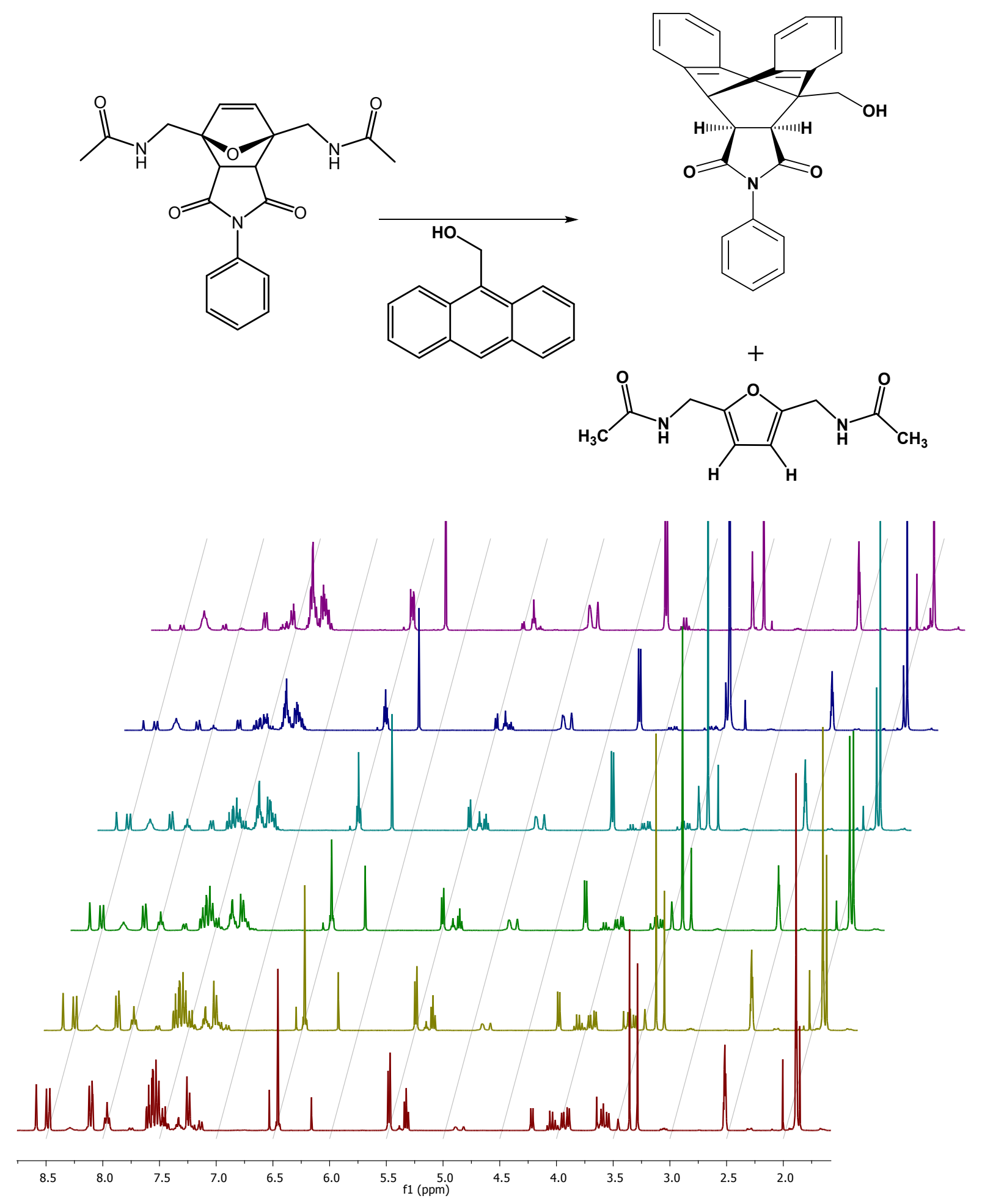


${ }^{1} \mathrm{H}$ NMR of in-situ exchange reaction for Compound $\mathbf{V}$ at $85^{\circ} \mathrm{C}$. Time increases from the bottom to the top of the figure.

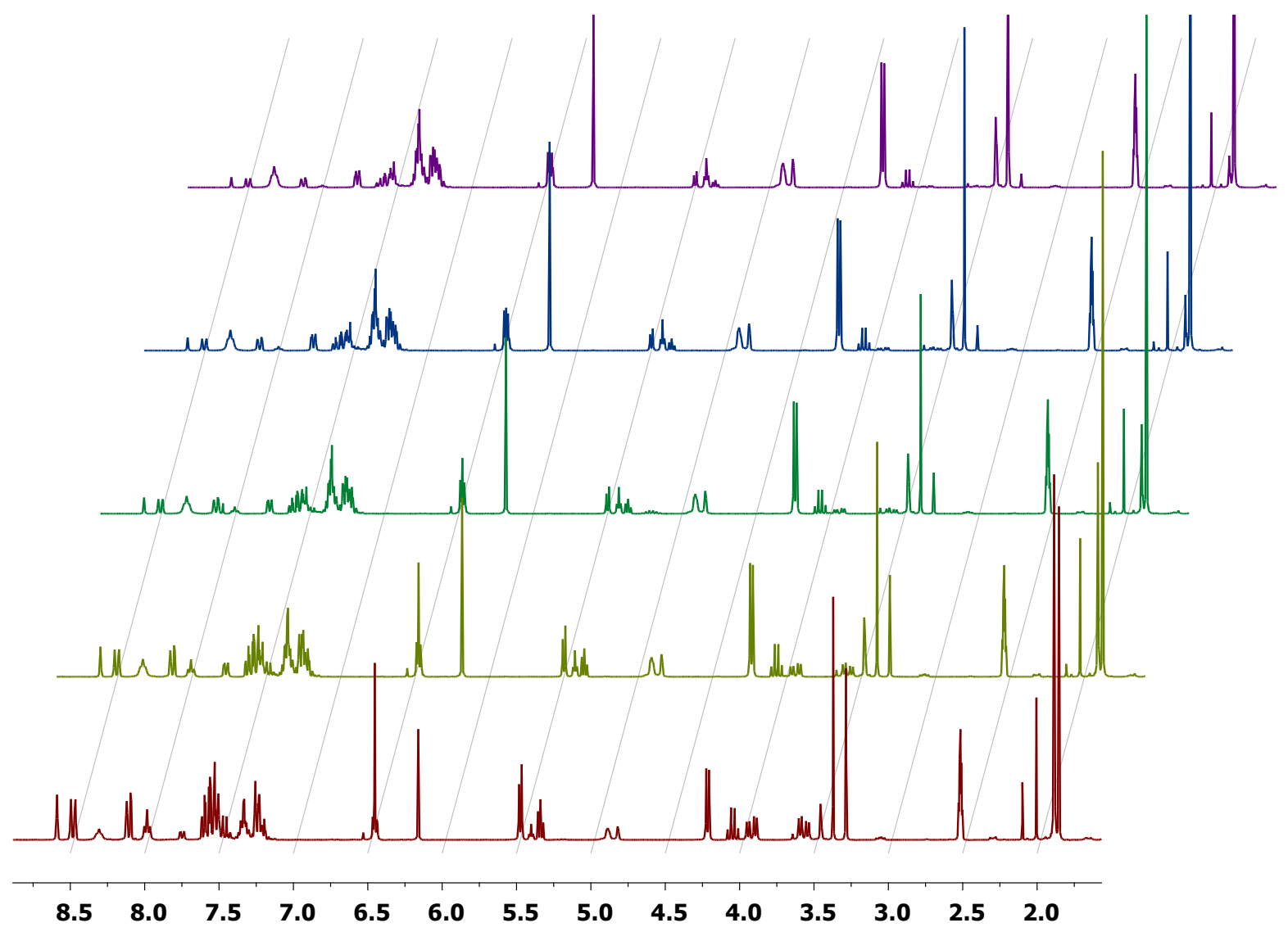


${ }^{1} \mathrm{H}$ NMR of $i n$-situ exchange reaction for Compound $\mathbf{V}$ at $105^{\circ} \mathrm{C}$. Time increases from the bottom to the top of the figure.

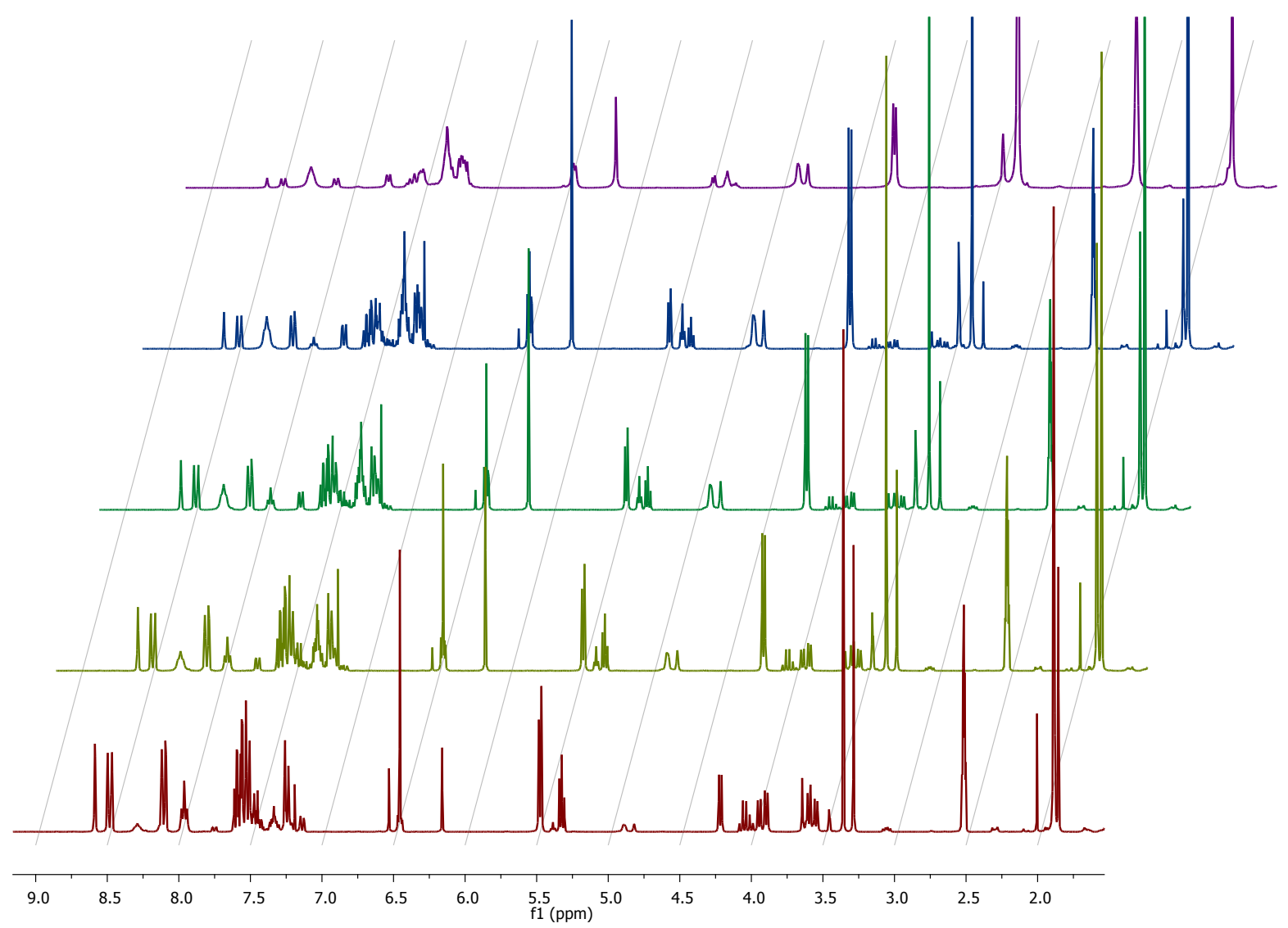


11. Compound VIII Exchange Reaction Kinetics

\begin{tabular}{cccc}
\hline Compound & Temperature & Time (min) & Exchange \% \\
\hline VIII & 190 & 30 & 8.3 \\
VIII & 190 & 90 & 26.3 \\
VIII & 190 & 360 & 71 \\
\hline VIII & 210 & 10 & 9.5 \\
VIII & 210 & 30 & 21.9 \\
VIII & 210 & 60 & 52.1 \\
VIII & 210 & 100 & 80.6 \\
VIII & 210 & 150 & 92.8 \\
VIII & 230 & 5 & 25.4 \\
VIII & 230 & 10 & 47.4 \\
VIII & 230 & 15 & 63.2 \\
VIII & 230 & 25 & 81.7 \\
VIII & 230 & 40 & 95.2 \\
\hline
\end{tabular}


${ }^{1} \mathrm{H}$ NMR of in-situ exchange reaction for Compound VIII at $190^{\circ} \mathrm{C}$. Time increases from the bottom to the top of the figure. Exchange percentage was measure by comparing the peak at 4.9 ppm which belong to the hydrogens of the carbons between the two benzene rings of Compound VIII, and the same peaks that shift slightly to $5.0 \mathrm{ppm}$ when the anthracene reacts with the Nphenylmaleimide.
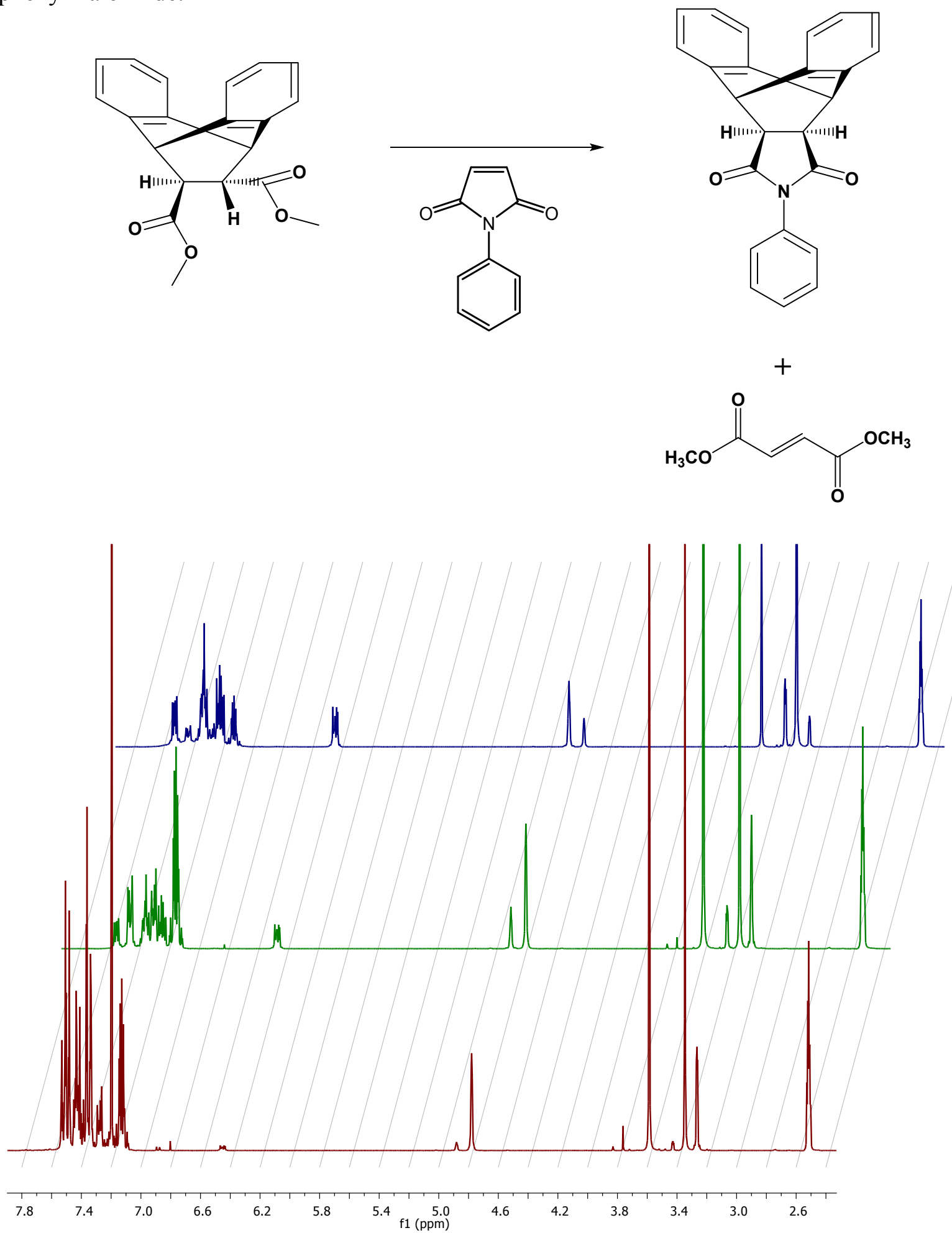
${ }^{1} \mathrm{H}$ NMR of in-situ exchange reaction for Compound VIII at $210^{\circ} \mathrm{C}$. Time increases from the bottom to the top of the figure.

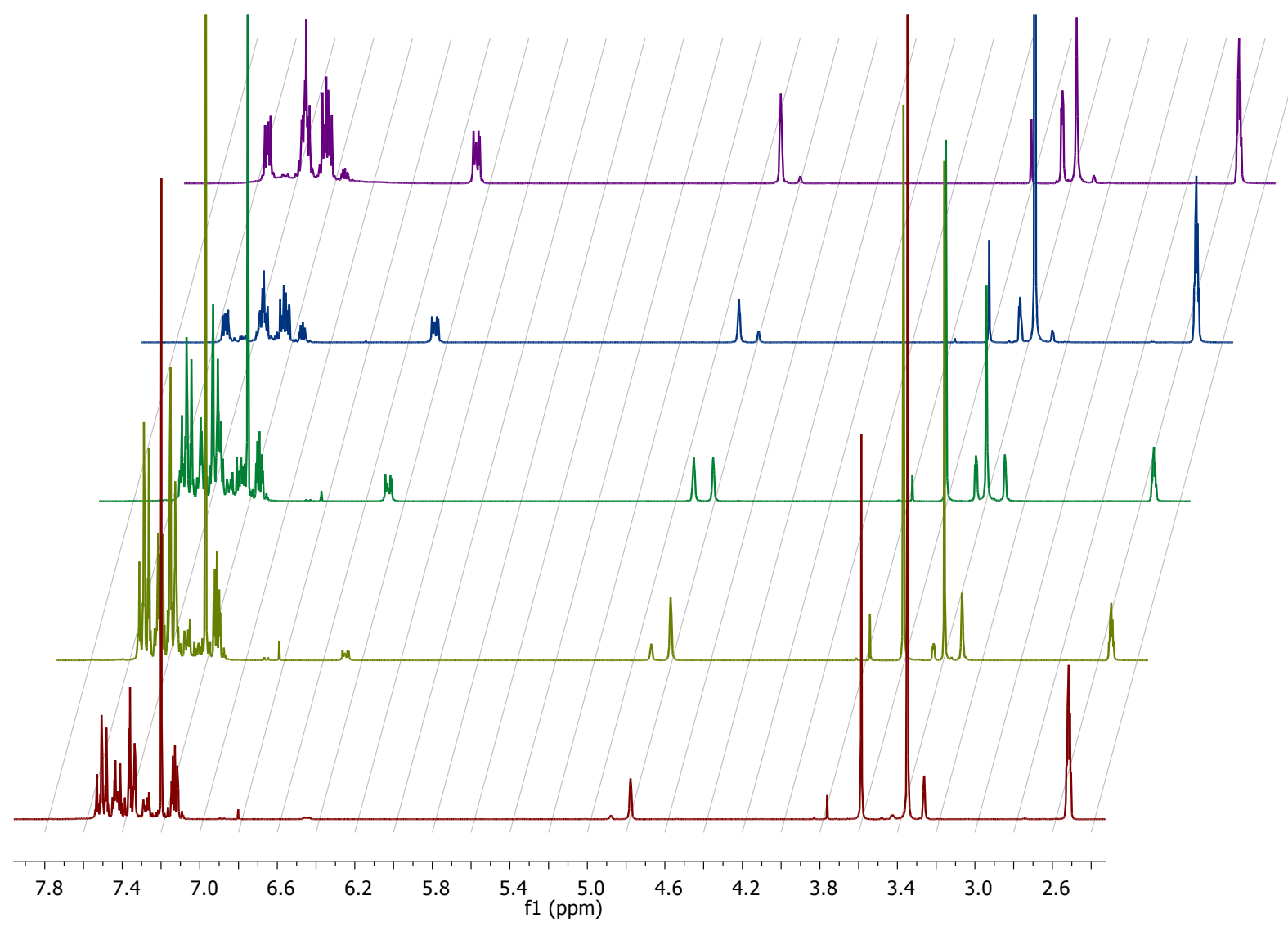


${ }^{1} \mathrm{H}$ NMR of in-situ exchange reaction for Compound VIII at $230^{\circ} \mathrm{C}$. Time increases from the bottom to the top of the figure.

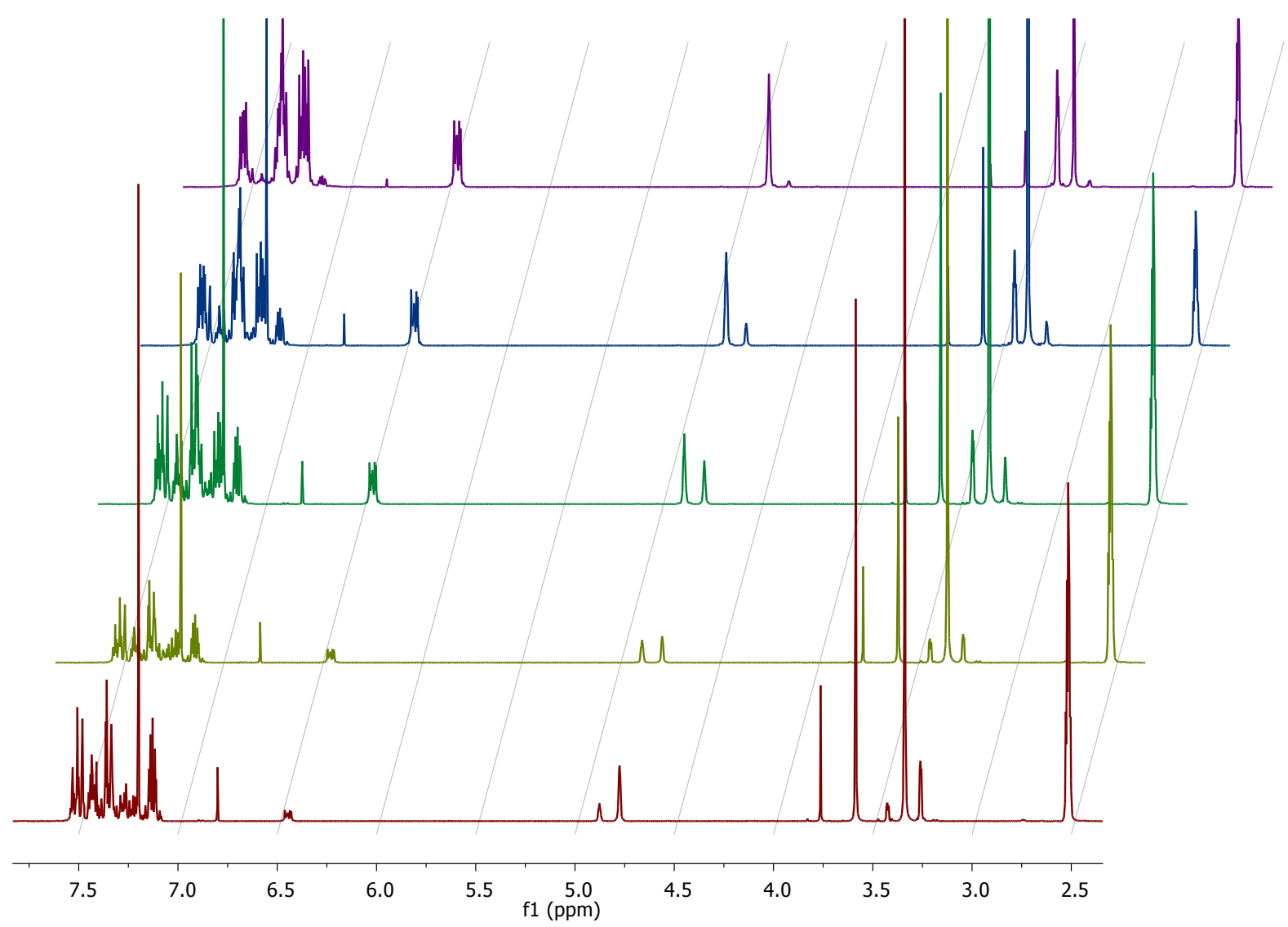




\section{Computational Data}

\begin{tabular}{|c|c|c|c|}
\hline \multicolumn{4}{|c|}{ Imaginary Frequencies $=0$} \\
\hline \multicolumn{4}{|c|}{ Energy (BP86/def-TZVP, level of optimization) $=-728.3891880349 \mathrm{H}$} \\
\hline \multicolumn{4}{|c|}{$\begin{array}{l}\text { Energy (M06-2X/def2-TZVP) }=-728.0676687366 \mathrm{H} \\
\mathrm{C}-3.5079452 \quad 0.3790706 \quad 0.1309716\end{array}$} \\
\hline $\mathrm{C}$ & -3.5079452 & 0.3790706 & 0.1309716 \\
\hline $\mathrm{C}$ & -2.7730622 & -0.8384714 & 0.0505269 \\
\hline C & -1.4007385 & -0.8076556 & -0.0174209 \\
\hline $\mathrm{C}$ & -0.6912018 & 0.4350633 & -0.0119025 \\
\hline $\mathrm{C}$ & -1.4368148 & 1.6782036 & 0.0486713 \\
\hline $\mathrm{C}$ & -2.8643885 & 1.5958875 & 0.1327980 \\
\hline C & -0.7216472 & 2.9027091 & 0.0687144 \\
\hline C & 0.6961220 & 2.9330694 & 0.0371422 \\
\hline C & 1.4197029 & 1.6787705 & -0.0432434 \\
\hline C & 0.7089255 & 0.4706826 & -0.0630618 \\
\hline C & 2.8491324 & 1.6980055 & -0.1018967 \\
\hline C & 3.5411242 & 2.8855356 & -0.0912898 \\
\hline C & 2.8337344 & 4.1199510 & -0.0234746 \\
\hline C & 1.4585154 & 4.1456467 & 0.0359963 \\
\hline $\mathrm{H}$ & -4.5968360 & 0.3448587 & 0.1962548 \\
\hline $\mathrm{H}$ & -3.3046349 & -1.7914550 & 0.0478296 \\
\hline $\mathrm{H}$ & -0.8235430 & -1.7334723 & -0.0694232 \\
\hline $\mathrm{H}$ & -3.4525713 & 2.5108628 & 0.1954763 \\
\hline C & -1.4646802 & 4.2014238 & 0.1611777 \\
\hline $\mathrm{H}$ & 1.2620936 & -0.4703210 & -0.1180984 \\
\hline $\mathrm{H}$ & 3.3779490 & 0.7442605 & -0.1601515 \\
\hline $\mathrm{H}$ & 4.6312509 & 2.8891690 & -0.1377443 \\
\hline $\mathrm{H}$ & 3.3914443 & 5.0581562 & -0.0219830 \\
\hline $\mathrm{H}$ & 0.9422849 & 5.1040155 & 0.0899257 \\
\hline 0 & -2.2966408 & 4.4047878 & -0.8894840 \\
\hline 0 & -1.3402728 & 5.0132959 & 1.0693208 \\
\hline $\mathrm{H}$ & -2.7626423 & 5.2590398 & -0.7466715 \\
\hline
\end{tabular}

\section{Diene 2}

Imaginary Frequencies $=0$

Energy (BP86/def-TZVP, level of optimization) $=-539.7245088013 \mathrm{H}$ Energy (M0 6-2X/def2-TZVP) $=-539.4848492069 \mathrm{H}$

$\begin{array}{lrrr}\text { C } & -2.0638941 & -3.8016336 & 0.1145255 \\ \text { C } & -1.3597709 & -5.0405141 & 0.0377399 \\ \text { C } & 0.0133334 & -5.0584533 & -0.0542849 \\ \text { C } & 0.7640281 & -3.8397527 & -0.0740470 \\ \text { C } & 0.0477711 & -2.5798597 & 0.0039388 \\ \text { C } & -1.3801108 & -2.6072746 & 0.0975898 \\ \text { C } & 0.7738478 & -1.3773779 & -0.0125573 \\ \text { C } & 2.1754998 & -1.3633561 & -0.1035381 \\ \text { C } & 2.8917831 & -2.6231924 & -0.1821180 \\ \text { C } & 2.1657899 & -3.8258771 & -0.1653019 \\ \text { C } & 4.3198494 & -2.5959787 & -0.2746935 \\ \text { C } & 5.0038271 & -1.4017324 & -0.2890093 \\ \text { C } & 4.2996356 & -0.1629275 & -0.2115961 \\ \text { C } & 2.9263965 & -0.1448724 & -0.1213026\end{array}$




$\begin{array}{rrrr}\text { H } & -3.1529600 & -3.8075981 & 0.1871302 \\ \mathrm{H} & -1.9198090 & -5.9773378 & 0.0523349 \\ \mathrm{H} & 0.5535615 & -6.0063747 & -0.1130965 \\ \mathrm{H} & -1.9191420 & -1.6588978 & 0.1562793 \\ \mathrm{H} & 0.2345834 & -0.4282360 & 0.0469683 \\ \mathrm{H} & 2.7052808 & -4.7750652 & -0.2241479 \\ \mathrm{H} & 4.8581115 & -3.5448078 & -0.3340451 \\ \mathrm{H} & 6.0929807 & -1.3955978 & -0.3600763 \\ \mathrm{H} & 4.8596958 & 0.7739285 & -0.2242896 \\ \mathrm{H} & 2.3868713 & 0.8033393 & -0.0617824\end{array}$

\section{Diene 3}

Imaginary Frequencies $=0$ Energy (BP86/def-TZVP, level of optimization) $=-654.3032248670 \mathrm{H}$ Energy (M06-2X/def2-TZVP) $=-654.0108607013 \mathrm{H}$

$\begin{array}{rrrr}\text { C } & -2.0592640 & -3.8350494 & 0.1340553 \\ \mathrm{C} & -1.3428941 & -5.0632184 & 0.0479371 \\ \mathrm{C} & 0.0271279 & -5.0486101 & -0.0589427 \\ \mathrm{C} & 0.7530588 & -3.8149540 & -0.0881355 \\ \mathrm{C} & 0.0301704 & -2.5563555 & -0.0130217 \\ \mathrm{C} & -1.3984007 & -2.6278133 & 0.1004347 \\ \mathrm{C} & 0.7483626 & -1.3311956 & -0.0565117 \\ \mathrm{C} & 2.1675851 & -1.3417859 & -0.1204807 \\ \mathrm{C} & 2.8768380 & -2.6051578 & -0.1943760 \\ \mathrm{C} & 2.1509404 & -3.8045233 & -0.1809334 \\ \mathrm{C} & 4.3056119 & -2.6070588 & -0.2769738 \\ \mathrm{C} & 5.0161635 & -1.4300893 & -0.2790554 \\ \mathrm{C} & 4.3280824 & -0.1857693 & -0.1961113 \\ \mathrm{C} & 2.9535348 & -0.1430125 & -0.1207397 \\ \mathrm{H} & -3.1466890 & -3.8530486 & 0.2267313 \\ \mathrm{H} & -1.8857284 & -6.0097272 & 0.0707865 \\ \mathrm{H} & 0.5909960 & -5.9823585 & -0.1205791 \\ \mathrm{H} & -1.9825122 & -1.7109405 & 0.1747185 \\ \mathrm{C} & 0.0152349 & -0.0087342 & 0.0021527 \\ \mathrm{H} & 2.6883944 & -4.7546493 & -0.2398175 \\ \mathrm{H} & 4.8204350 & -3.5687653 & -0.3360428 \\ \mathrm{H} & 6.1056556 & -1.4431018 & -0.3408591 \\ \mathrm{H} & 4.8996253 & 0.7442519 & -0.1909321 \\ \mathrm{H} & 2.4581782 & 0.8243773 & -0.0422190 \\ \mathrm{O} & 0.0128147 & 0.5866760 & 1.3264878 \\ \mathrm{H} & -0.4242975 & -0.0436110 & 1.9283890 \\ \mathrm{H} & -1.0151540 & -0.1132453 & -0.3657640 \\ \mathrm{H} & 0.5044701 & 0.7323294 & -0.6412873 \\ & & & \end{array}$

\section{Diene 4}

Imaginary Frequencies $=0$

Energy (BP86/def-TZVP, level of optimization) $=-384.0686460031 \mathrm{H}$ Energy (M0 6-2X/def2-TZVP) $=-383.8740318619 \mathrm{H}$

$\begin{array}{llll}\text { H } & -6.3235635 & -1.4117612 & 0.1105558 \\ \mathrm{C} & -5.8433106 & -2.3955534 & 0.0238846 \\ \mathrm{H} & -6.1629628 & -3.0221984 & 0.8735049 \\ \mathrm{H} & -6.2247885 & -2.8978391 & -0.8808633 \\ \mathrm{C} & -4.3570807 & -2.2929334 & -0.0204392\end{array}$




$\begin{array}{lrrr}\text { C } & -3.6532310 & -1.1367849 & 0.0410916 \\ \text { C } & -2.2144152 & -1.0948263 & -0.0037894 \\ \text { C } & -1.4762741 & 0.0437550 & 0.0559611 \\ \text { C } & -0.0134128 & 0.0149909 & 0.0072047 \\ \text { O } & 0.5148293 & 1.2747218 & 0.0768054 \\ \text { O } & 0.7016325 & -0.9837648 & -0.0836567 \\ \text { H } & -3.8022147 & -3.2344534 & -0.1094038 \\ \text { H } & -4.1864711 & -0.1837123 & 0.1301120 \\ \text { H } & -1.6865023 & -2.0511702 & -0.0918167 \\ \text { H } & -1.9535153 & 1.0217668 & 0.1430550 \\ \text { H } & 1.4915207 & 1.1881230 & 0.0387339\end{array}$

\section{Diene 5}

Imaginary Frequencies $=0$

Energy (BP86/def-TZVP, level of optimization) $=-724.9882064958 \mathrm{H}$ Energy (M06-2X/def2-TZVP) $=-724.6652025170 \mathrm{H}$

$-0.7285825$

$-1.7536641$

0.0840075

C $\quad 0.7036228$

$-2.9688151$

$-0.3969667$

1.0800874

$-2.9370443$

$-0.4256135$

$-0.0409356$

$-1.7093180$

0.0522669

$-1.3745045$

1. 3726747

$-0.9715491$

0.3670153

$-3.7865773$

$-0.7023448$

$-2.4850450$

$-3.7276181$

$-0.7520543$

$-1.1534815$

0.3089446

$-3.2417120$

$-1.8638345$

$-2.6093409$

$-0.2132506$

$-0.0619778$

2.4073510

$-1.0807988$

$-0.2516760$

0.3028787

2. 5207554

$-0.7732704$

1.3549250

3.1856678

$-1.8321466$

0.0918113

$-2.7367791$

$-0.8037814$

1.7054066

2. 6266822

0.1343031

$-0.4788272$

0.4568456

2.0790681

3. 0164508

1. 3165709

0.1067603

3.1011355

2.5095467

$-0.8322739$

2. 8804758

2. 2681955

$-1.8811107$

2.3933850

3. 2753913

$-0.4868196$

4.1093286

2. 9393894

$-0.7668686$

3. 2822542

1. 4053246

1. 3050480

2. 3740667

0.1261358

$-1.4622212$

$-3.2599894$

0.6830188

3. 5779659

$-2.5431604$

1. 4626351

3.8702180

$-4.2665270$

1.0610583

3. 7996381

$-3.0726393$

$-0.2161252$

4.1808997

$-3.3930196$

1.3408071

1.2607439

$-2.4969636$

$-1.4816745$

2.4223416

\section{Dienophile A}

Imaginary Frequencies $=0$

Energy (BP86/def-TZVP, level of optimization) $=-307.7936350432 \mathrm{H}$

Energy (M06-2X/def2-TZVP) $=-307.6328165222 \mathrm{H}$

$\begin{array}{llll}\mathrm{C} & 0.6264745 & -1.1715956 & -0.6923692 \\ \mathrm{C} & 1.6339454 & -1.9405660 & -0.2430673 \\ \mathrm{H} & 0.2236675 & -1.3702917 & -1.6918598\end{array}$




$\begin{array}{rrrr}\text { H } & 2.0137479 & -2.7364301 & -0.8936996 \\ \mathrm{C} & -0.0341407 & -0.0293487 & 0.0224070 \\ \mathrm{H} & 0.2973285 & 0.0468071 & 1.0710963 \\ \mathrm{H} & 0.2162479 & 0.9221312 & -0.4717684 \\ \mathrm{C} & 2.3361133 & -1.8369399 & 1.0792231 \\ \mathrm{H} & 3.3685783 & -1.4839428 & 0.9330200 \\ \mathrm{H} & 1.8365964 & -1.1235122 & 1.7554985 \\ \mathrm{O} & 2.4706631 & -3.1304162 & 1.7166208 \\ \mathrm{H} & 1.5695507 & -3.4712401 & 1.8667002 \\ \mathrm{O} & -1.4779540 & -0.1177548 & -0.0482487 \\ \mathrm{H} & -1.7364761 & -0.9509708 & 0.3873554\end{array}$

\section{Dienophile B}

Imaginary Frequencies $=0$

Energy (BP86/def-TZVP, level of optimization) $=-590.7312852639 \mathrm{H}$

Energy (M06-2X/def2-TZVP) $=-590.46555743432 \mathrm{H}$

$3.3510712-0.1834849$

$-0.6427738$

3.3508913

0.1874603

$\mathrm{H} \quad 1.3040061$

4.1940767

$-0.3762457$

1.1082215

1.9334225

$-0.3246124$

$-1.3015189$

4.1937076

0.3823240

$-1.1066328$

1.9331330

0.3251089

$-2.2106956$

1.5318238

0.6611283

2. 2120271

1.5324898

$-0.6616205$

1.1178193

$-0.0007761$

0.0003153

$-0.3115741$

$-0.0017409$

$-1.0054947$

0.7980206

0.9177033

$-2.4034657$

0.7871846

$-0.0006089$

$-3.1035736$

$-0.0029478$

$-2.4021593$

$-0.7925308$

$-1.0041750$

$-0.8021869$

$-0.4551733$

1.4256238

$-2.9457730$

1.4061139

1.6354121

$-2.9434049$

$-1.4118720$

$-1.6184474$

$-0.4528270$

$-1.4292461$

$-0.0010760$

$-4.1948047$

$-0.0034204$

\section{Dienophile C}

Imaginary Frequencies $=0$

Energy (BP86/def-TZVP, level of optimization) $=-630.0677957663 \mathrm{H}$

Energy (M06-2X/def2-TZVP) $=-629.7822522142 \mathrm{H}$

$\begin{array}{lrrr}\text { C } & 0.6445539 & 3.3706736 & -0.1514550 \\ \mathrm{C} & -0.6682182 & 3.3482237 & 0.1467970 \\ \mathrm{C} & 1.1151574 & 1.9359659 & -0.2591517 \\ \mathrm{H} & -1.3426364 & 4.1874513 & 0.3018721 \\ \mathrm{C} & -1.1296319 & 1.9394971 & 0.2614449 \\ \mathrm{O} & -2.2479334 & 1.5267301 & 0.5356351 \\ \mathrm{O} & 2.2401258 & 1.5438862 & -0.5311303 \\ \mathrm{~N} & -0.0012381 & 1.1197713 & 0.0016273 \\ \mathrm{C} & 0.0012788 & -0.3086902 & 0.0077211 \\ \mathrm{C} & 0.8841038 & -0.9975202 & 0.8502095 \\ \mathrm{C} & 0.8894102 & -2.3954723 & 0.8458969 \\ \mathrm{C} & 0.0072882 & -3.1018103 & 0.0193435\end{array}$




$\begin{array}{lrrr}\text { C } & -0.8775830 & -2.4061326 & -0.8132629 \\ \mathrm{C} & -0.8782039 & -1.0081935 & -0.8294269 \\ \mathrm{H} & 1.5556779 & -0.4426428 & 1.5057144 \\ \mathrm{H} & 1.5791820 & -2.9331032 & 1.4983625 \\ \mathrm{H} & -1.5649122 & -2.9521151 & -1.4613621 \\ \mathrm{H} & -1.5518655 & -0.4615993 & -1.4896641 \\ \mathrm{H} & 0.0095457 & -4.1930516 & 0.0239463 \\ \mathrm{C} & 1.5734197 & 4.5078523 & -0.3666696 \\ \mathrm{H} & 2.0344283 & 4.4464042 & -1.3646721 \\ \mathrm{H} & 1.0538718 & 5.4683094 & -0.2708859 \\ \mathrm{H} & 2.3992290 & 4.4734161 & 0.3614799\end{array}$

\section{Dienophile D}

Imaginary Frequencies $=0$

Energy (BP86/def-TZVP, level of optimization) $=-746.8226071259 \mathrm{H}$

Energy (M06-2X/def2-TZVP) $=-746.5101692140 \mathrm{H}$

$\begin{array}{rrrr}\mathrm{H} & -1.1579254 & 1.2839611 & 1.6049231 \\ \mathrm{H} & 1.6415745 & 1.2908263 & 0.9975783 \\ \mathrm{C} & -1.0811165 & 2.3326333 & 1.9433083 \\ \mathrm{C} & 1.6998242 & 2.3426801 & 1.3291733 \\ \mathrm{C} & 1.2437542 & 2.4495024 & 2.7614313 \\ \mathrm{H} & -2.0843155 & 2.6287355 & 2.2792686 \\ \mathrm{C} & -0.0653609 & 2.4406887 & 3.0512910 \\ \mathrm{H} & 2.7475968 & 2.6508875 & 1.2097572 \\ \mathrm{C} & 0.8185938 & 3.2000559 & 0.3843834 \\ \mathrm{H} & 0.9894770 & 2.8676700 & -0.6512262 \\ \mathrm{C} & 1.2533279 & 4.6670565 & 0.4362312 \\ \mathrm{C} & -0.6847379 & 3.2098441 & 0.7298743 \\ \mathrm{C} & -1.0269171 & 4.6788434 & 0.9965272 \\ \mathrm{H} & -1.2950118 & 2.9097235 & -0.1362451 \\ \mathrm{O} & 2.3728765 & 5.0956746 & 0.2294519 \\ \mathrm{O} & -2.1090513 & 5.1165234 & 1.3390608 \\ \mathrm{~N} & 0.1299008 & 5.4568672 & 0.7680409 \\ \mathrm{C} & 0.1704529 & 6.8839321 & 0.8896070 \\ \mathrm{C} & -0.3894690 & 7.5049769 & 2.0127885 \\ \mathrm{C} & -0.3502548 & 8.8968406 & 2.1189104 \\ \mathrm{C} & 0.2502903 & 9.6660941 & 1.1180507 \\ \mathrm{C} & 0.8119890 & 9.0375150 & 0.0026613 \\ \mathrm{C} & 0.7714147 & 7.6473171 & -0.1189599 \\ \mathrm{H} & -0.8588249 & 6.9028719 & 2.7887158 \\ \mathrm{H} & -0.7911832 & 9.3802331 & 2.9920398 \\ \mathrm{H} & 0.2813396 & 10.7531050 & 1.2071764 \\ \mathrm{H} & 1.2845093 & 9.6309669 & -0.7815249 \\ \mathrm{H} & 1.2116811 & 7.1546268 & -0.9844801 \\ \mathrm{H} & 1.9948743 & 2.5232377 & 3.5507800 \\ \mathrm{H} & -0.4138686 & 2.5064494 & 4.0842056\end{array}$

\section{Dienophile E}

Imaginary Frequencies $=0$

Energy (BP86/def-TZVP, level of optimization) $=-534.5920396032 \mathrm{H}$

Energy (M0 6-2X/def2-TZVP) $=-534.3420636703 \mathrm{H}$

C $\quad 0.4347708 \quad-1.4254150 \quad-0.0201017$

$\mathrm{H} \quad-0.3445492 \quad-2.1875577 \quad-0.0743362$ 


$\begin{array}{rrrr}\mathrm{C} & 1.7329291 & -1.7637120 & 0.0201789 \\ \mathrm{H} & 2.5122108 & -1.0016019 & 0.0755035 \\ \mathrm{C} & 0.0076469 & -0.0042804 & 0.0096340 \\ \mathrm{O} & -1.3426451 & 0.0812240 & -0.0274294 \\ \mathrm{C} & 2.1600632 & -3.1849029 & -0.0080410 \\ \mathrm{O} & 3.5096807 & -3.2707916 & 0.0485204 \\ \mathrm{O} & 0.7541482 & 0.9658385 & 0.0604841 \\ \mathrm{O} & 1.4141720 & -4.1546453 & -0.0729131 \\ \mathrm{C} & -1.8947284 & 1.4279261 & 0.0000100 \\ \mathrm{H} & -2.9789522 & 1.2935158 & -0.0379372 \\ \mathrm{H} & -1.5985345 & 1.9379500 & 0.9252452 \\ \mathrm{H} & -1.5429482 & 1.9976883 & -0.8691714 \\ \mathrm{C} & 4.0620660 & -4.6174508 & 0.0254639 \\ \mathrm{H} & 5.1452901 & -4.4834573 & 0.0865425 \\ \mathrm{H} & 3.6922768 & -5.1919270 & 0.8839549 \\ \mathrm{H} & 3.7851332 & -5.1222609 & -0.9085872\end{array}$

\section{TS $1+\mathrm{A}$}

Imaginary Frequencies $=1(-366.82 \mathrm{~cm}-1)$

Energy(BP86/def-TZVP, level of optimization) $=-1035.0429694654 \mathrm{H}$

Energy (M06-2X/def2-TZVP) $=-1035.666749329 \mathrm{H}$

$\begin{array}{lrrr}\text { C } & -1.0758770 & -0.2413406 & 0.5788357 \\ \mathrm{C} & -0.1540597 & -0.3095392 & 1.7048824 \\ \mathrm{C} & 1.2300364 & -0.5325753 & 1.3966406 \\ \mathrm{C} & 1.6077331 & -0.5478287 & -0.0076017 \\ \mathrm{C} & -2.5158511 & 0.1112563 & 0.7676084 \\ \mathrm{H} & 2.6751005 & -0.7178648 & -0.2261395 \\ \mathrm{C} & 0.2478485 & 1.8791966 & -0.4383205 \\ \mathrm{C} & 1.5803428 & 1.3880903 & -0.4372750 \\ \mathrm{C} & -0.5092474 & 2.0848070 & -1.7151027 \\ \mathrm{H} & -0.1561749 & 2.3637172 & 0.4637039 \\ \mathrm{H} & 2.1760029 & 1.6920858 & 0.4421497 \\ \mathrm{C} & 2.3908258 & 1.4379999 & -1.7361647 \\ \mathrm{O} & 3.6944042 & 0.8879172 & -1.6253002 \\ \mathrm{C} & -0.7327820 & -0.9054334 & -0.6672166 \\ \mathrm{C} & 0.6591570 & -1.1268390 & -0.9414180 \\ \mathrm{C} & 2.1987216 & -0.5888274 & 2.4298006 \\ \mathrm{C} & 1.8243110 & -0.4382879 & 3.7647866 \\ \mathrm{C} & 0.4668443 & -0.1858482 & 4.0795974 \\ \mathrm{C} & -0.5000693 & -0.1107305 & 3.0770989 \\ \mathrm{C} & -1.6844595 & -1.3492347 & -1.6343282 \\ \mathrm{C} & -1.2722509 & -2.0206297 & -2.7864408 \\ \mathrm{C} & 0.1016427 & -2.2513317 & -3.0394342 \\ \mathrm{C} & 1.0559892 & -1.7878351 & -2.1315327 \\ \mathrm{H} & 3.2541520 & -0.7562089 & 2.1613275 \\ \mathrm{H} & 2.5771217 & -0.5030782 & 4.5652149 \\ \mathrm{H} & 0.1652547 & -0.0477491 & 5.1295912 \\ \mathrm{H} & -1.5428790 & 0.0835611 & 3.3533133 \\ \mathrm{H} & -2.7573039 & -1.1796445 & -1.4662294 \\ \mathrm{H} & -2.0281143 & -2.3751629 & -3.5042365 \\ \mathrm{H} & 0.4148854 & -2.7840306 & -3.9500732 \\ \mathrm{H} & 2.1298661 & -1.9425256 & -2.3217434 \\ \mathrm{O} & -3.0938721 & -0.4102833 & 1.8623902\end{array}$




$\begin{array}{rrrr}\mathrm{O} & -3.1784705 & 0.7926388 & -0.0239262 \\ \mathrm{H} & 4.1991022 & 1.4473882 & -1.0021032 \\ \mathrm{O} & -1.8272978 & 2.5551318 & -1.5666083 \\ \mathrm{H} & -2.3288792 & 1.8684029 & -1.0503841 \\ \mathrm{H} & -4.0277960 & -0.0928562 & 1.8829195 \\ \mathrm{H} & -0.4571437 & 1.1383857 & -2.3202798 \\ \mathrm{H} & 0.0425627 & 2.8358027 & -2.3346299 \\ \mathrm{H} & 2.4328762 & 2.5003506 & -2.0778428 \\ \mathrm{H} & 1.8755729 & 0.8661627 & -2.5353965\end{array}$

\section{Product $1+\mathbf{A}$}

Imaginary Frequencies $=0$

Energy (BP86/def-TZVP, level of optimization) $=-1036.1966634781 \mathrm{H}$

Energy (M0 6-2X/def2-TZVP) $=-1035.747695374 \mathrm{H}$

$\begin{array}{lrrr}\text { C } & 1.4460485 & -0.3036962 & -0.5002383 \\ \text { C } & 2.2208240 & -0.6558893 & 0.7843843 \\ \text { C } & 3.5489256 & -1.0450462 & 0.5369211 \\ \text { C } & 3.9292347 & -0.9914802 & -0.9223409 \\ \text { C } & 0.0117753 & 0.0524541 & -0.1119501 \\ \text { H } & 4.9643183 & -1.3169957 & -1.0901080 \\ \text { C } & 2.2193592 & 0.8902113 & -1.2147592 \\ \mathrm{C} & 3.7415821 & 0.5095838 & -1.3546974 \\ \mathrm{C} & 1.4939634 & 1.2468886 & -2.5180000 \\ \mathrm{H} & 2.1357367 & 1.7648784 & -0.5566701 \\ \mathrm{H} & 4.3053480 & 1.1151561 & -0.6287741 \\ \mathrm{C} & 4.4054946 & 0.7584447 & -2.7175855 \\ \mathrm{O} & 4.5748266 & 2.1518542 & -3.0251004 \\ \mathrm{C} & 1.5845750 & -1.5015712 & -1.4525800 \\ \mathrm{C} & 2.9347205 & -1.8392029 & -1.6859859 \\ \mathrm{C} & 4.3888277 & -1.4004198 & 1.5942094 \\ \mathrm{C} & 3.8972433 & -1.3761087 & 2.9067290 \\ \mathrm{C} & 2.5756279 & -0.9898241 & 3.1537994 \\ \mathrm{C} & 1.7325499 & -0.6265349 & 2.0933374 \\ \mathrm{C} & 0.5717383 & -2.1952997 & -2.1223445 \\ \mathrm{C} & 0.9080941 & -3.2341775 & -3.0028733 \\ \mathrm{C} & 2.2464810 & -3.5756727 & -3.2237799 \\ \mathrm{C} & 3.2642929 & -2.8724482 & -2.5661210 \\ \mathrm{H} & 5.4210354 & -1.6981189 & 1.3953002 \\ \mathrm{H} & 4.5472294 & -1.6607353 & 3.7361576 \\ \mathrm{H} & 2.1927063 & -0.9721931 & 4.1756178 \\ \mathrm{H} & 0.7040437 & -0.3293869 & 2.3075980 \\ \mathrm{H} & -0.4745813 & -1.9431724 & -1.9577811 \\ \mathrm{H} & 0.1140220 & -3.7787688 & -3.5171429 \\ \mathrm{H} & 2.4992217 & -4.3879767 & -3.9078581 \\ \mathrm{H} & 4.3125571 & -3.1283500 & -2.7370407 \\ \mathrm{O} & -0.1380409 & 1.3680007 & 0.1705561 \\ \mathrm{O} & -0.8995180 & -0.7506997 & 0.0166218 \\ \mathrm{H} & 3.6567024 & 2.5215585 & -3.0821902 \\ \mathrm{O} & 1.8885536 & 2.5866612 & -2.9131386 \\ \mathrm{H} & 1.4565684 & 2.7829308 & -3.7652742 \\ \mathrm{H} & -1.0653120 & 1.5080891 & 0.4642815 \\ \mathrm{H} & 0.4044164 & 1.2350330 & -2.3583174 \\ \mathrm{H} & 1.7251896 & 0.5321171 & -3.3217985 \\ & & & \end{array}$




$\begin{array}{llll}\mathrm{H} & 3.8596210 & 0.2427977 & -3.5284199 \\ \mathrm{H} & 5.4179909 & 0.3302069 & -2.6856333\end{array}$

\section{TS $2+A$}

Imaginary Frequencies $=1(-414.67 \mathrm{~cm}-1)$

Energy (BP86/def-TZVP, level of optimization) $=-847.4729040211 \mathrm{H}$

Energy (M06-2X/def2-TZVP) $=-847.0886188084 \mathrm{H}$

$\begin{array}{llll}\mathrm{C} & -0.4952006 & 1.3325206 & 0.3434517\end{array}$

C $\quad-0.5291734 \quad 0.6834959 \quad 1.6260864$

C $\quad-0.4607381 \quad-0.7421450 \quad 1.6279263$

$\begin{array}{llll}\text { C } & -0.3701101 & -1.3896743 & 0.3484031\end{array}$

$\mathrm{H} \quad-0.4596135 \quad 2.4241431 \quad 0.3213588$

$\mathrm{H} \quad-0.2284575 \quad-2.4724753 \quad 0.3280183$

$\begin{array}{llll}\text { C } & 1.6437638 & 0.7893735 & -0.1087598\end{array}$

C $\quad 1.7219417 \quad-0.6180231 \quad-0.1161595$

C $\quad 1.8840902 \quad 1.5923151 \quad-1.3641176$

$\mathrm{H} \quad 1.9497282 \quad 1.2941431 \quad 0.8108896$

$\mathrm{H} \quad 2.0810072 \quad-1.1075960 \quad 0.7912112$

$\begin{array}{llll}\text { C } & 2.0181201 & -1.3724956 & -1.3786280\end{array}$

$\begin{array}{llll}0 & 1.9979873 & -2.7854103 & -1.1054337\end{array}$

C $\quad-1.0595539 \quad 0.6567844 \quad-0.7884290$

$\begin{array}{llll}\text { C } & -0.9907127 & -0.7727563 & -0.7865384\end{array}$

$\begin{array}{llll}\text { C } & -0.3911713 & -1.4312544 & 2.8626134\end{array}$

C $\quad-0.4193615 \quad-0.7297070 \quad 4.0607763$

$\begin{array}{llll}\text { C } & -0.4866375 & 0.6826027 & 4.0587133\end{array}$

$\begin{array}{llll}\mathrm{C} & -0.5254325 & 1.3799065 & 2.8581963\end{array}$

C $\quad-1.5781003 \quad 1.3234420 \quad-1.9253205$

$\begin{array}{llll}\mathrm{C} & -2.0514839 & 0.5972441 & -3.0105485\end{array}$

C $\quad-1.9813153 \quad-0.8143465 \quad-3.0096698$

C $\quad-1.4407979 \quad-1.4889117 \quad-1.9227574$

$\mathrm{H} \quad-0.3274073 \quad-2.5221217 \quad 2.8627561$

$\mathrm{H} \quad-0.3868744 \quad-1.2690292 \quad 5.0095526$

$\mathrm{H} \quad-0.5055790 \quad 1.2254463 \quad 5.0058313$

$\mathrm{H} \quad-0.5653001 \quad 2.4719023 \quad 2.8549714$

$\mathrm{H} \quad-1.6197372 \quad 2.4150139 \quad-1.9301464$

$\mathrm{H} \quad-2.4739298 \quad 1.1177590 \quad-3.8723329$

$\mathrm{H} \quad-2.3498246 \quad-1.3751318 \quad-3.8709413$

$\mathrm{H}-1.3742043 \quad-2.5792559 \quad-1.9257830$

$\mathrm{H} \quad 2.2224685 \quad-3.2420617 \quad-1.9359120$

$\begin{array}{llll}0 & 1.7201121 & 3.0098697 & -1.1886322\end{array}$

$\begin{array}{llll}\mathrm{H} & 2.3829619 & 3.3023674 & -0.5367004\end{array}$

$\mathrm{H} \quad 1.1781166 \quad 1.3051441 \quad-2.1582791$

$\mathrm{H} \quad 2.9033916 \quad 1.3797564 \quad-1.7450304$

$\mathrm{H} \quad 3.0191823 \quad-1.0800917 \quad-1.7563143$

$\mathrm{H} \quad 1.2855014 \quad-1.1170609 \quad-2.1670350$

\section{Product $2+\mathrm{A}$}

Imaginary Frequencies $=0$

Energy (BP86/def-TZVP, level of optimization) $=-847.5370140881 \mathrm{H}$

Energy (M06-2X/def2-TZVP) $=-847.1631900948 \mathrm{H}$

$\begin{array}{llrl}\mathrm{C} & -3.3645821 & 0.2862871 & 1.8572994 \\ \mathrm{C} & -3.7092887 & -0.3161011 & 3.2053899 \\ \mathrm{C} & -3.6728716 & -1.7237309 & 3.2055206\end{array}$




$\begin{array}{rrrr}\mathrm{C} & -3.3023811 & -2.3080901 & 1.8562468 \\ \mathrm{H} & -3.4223028 & 1.3828379 & 1.8689813 \\ \mathrm{H} & -3.3071600 & -3.4059665 & 1.8678905 \\ \mathrm{C} & -1.8969632 & -0.1850571 & 1.5171668 \\ \mathrm{C} & -1.8623857 & -1.7636986 & 1.5064536 \\ \mathrm{C} & -1.3981601 & 0.5377514 & 0.2553719 \\ \mathrm{H} & -1.2566237 & 0.1643989 & 2.3417178 \\ \mathrm{H} & -1.1946376 & -2.1018786 & 2.3135623 \\ \mathrm{C} & -1.3724984 & -2.4455242 & 0.2293536 \\ \mathrm{O} & 0.0589561 & -2.2325459 & 0.0914895 \\ \mathrm{C} & -4.2968440 & -0.3286141 & 0.8309711 \\ \mathrm{C} & -4.2654195 & -1.7378093 & 0.8318154 \\ \mathrm{C} & -3.9458641 & -2.4363330 & 4.3746114 \\ \mathrm{C} & -4.2652963 & -1.7361508 & 5.5487500 \\ \mathrm{C} & -4.3012170 & -0.3361283 & 5.5490234 \\ \mathrm{C} & -4.0182332 & 0.3804375 & 4.3754892 \\ \mathrm{C} & -5.1292810 & 0.3550079 & -0.0581276 \\ \mathrm{C} & -5.9375496 & -0.3723013 & -0.9455684 \\ \mathrm{C} & -5.9059177 & -1.7722167 & -0.9446783 \\ \mathrm{C} & -5.0655284 & -2.4605595 & -0.0558863 \\ \mathrm{H} & -3.9146317 & -3.5285200 & 4.3751703 \\ \mathrm{H} & -4.4878147 & -2.2866750 & 6.4649479 \\ \mathrm{H} & -4.5519052 & 0.2016418 & 6.4655464 \\ \mathrm{H} & -4.0433074 & 1.4727790 & 4.3770036 \\ \mathrm{H} & -5.1543963 & 1.4474990 & -0.0594443 \\ \mathrm{H} & -6.5949615 & 0.1570938 & -1.6379959 \\ \mathrm{H} & -6.5387395 & -2.3318003 & -1.6362600 \\ \mathrm{H} & -5.0408321 & -3.5531124 & -0.0553187 \\ \mathrm{H} & 0.3446723 & -2.6634837 & -0.7353258 \\ \mathrm{O} & 0.0291034 & 0.4862918 & 0.0907695 \\ \mathrm{H} & 0.2516346 & -0.4779635 & 0.0203118 \\ \mathrm{H} & -1.6530650 & 1.6044337 & 0.3442919 \\ \mathrm{H} & -1.9081444 & 0.1554454 & -0.6475665 \\ \mathrm{H} & -1.8969300 & -2.0687350 & -0.6635882 \\ \mathrm{H} & -1.5651599 & -3.5278883 & 0.3072776 \\ & & & \end{array}$

TS $3+\mathrm{A}$

Imaginary Frequencies $=1(-381.07 \mathrm{~cm}-1)$

Energy (BP86/def-TZVP, level of optimization) $=-962.0587559440 \mathrm{H}$

Energy (M06-2X/def2-TZVP) $=-961.6197949891 \mathrm{H}$

$\begin{array}{rrrr}\text { C } & -0.8232343 & 0.6473563 & 0.7445392 \\ \text { C } & 0.0260638 & 0.0910199 & 1.7779694 \\ \text { C } & 0.7816427 & -1.0737394 & 1.4532158 \\ \text { C } & 0.6601817 & -1.5760914 & 0.1085314 \\ \text { C } & -1.5288353 & 1.9593319 & 1.0101613 \\ \text { H } & 1.2665148 & -2.4471543 & -0.1550242 \\ \mathrm{C} & 1.0770203 & 1.2093544 & -0.6224427 \\ \mathrm{C} & 1.7506093 & -0.0111115 & -0.8566941 \\ \mathrm{H} & 1.4230859 & 1.8219633 & 0.2124346 \\ \mathrm{H} & 2.6210147 & -0.2034056 & -0.2248089 \\ \mathrm{C} & 1.8714680 & -0.5905942 & -2.2544751 \\ \mathrm{C} & -1.3743956 & -0.2667314 & -0.2303133 \\ \mathrm{C} & -0.6128237 & -1.4349961 & -0.5446023\end{array}$




$\begin{array}{rrrr}\mathrm{C} & 1.6860518 & -1.6154340 & 2.3945613 \\ \mathrm{C} & 1.8212827 & -1.0385018 & 3.6494854 \\ \mathrm{C} & 1.0742584 & 0.1135377 & 3.9788535 \\ \mathrm{C} & 0.2020626 & 0.6769640 & 3.0572656 \\ \mathrm{C} & -2.5886284 & -0.0450428 & -0.9327049 \\ \mathrm{C} & -3.0441338 & -0.9613478 & -1.8698571 \\ \mathrm{C} & -2.2895376 & -2.1165507 & -2.1694007 \\ \mathrm{C} & -1.0799496 & -2.3384328 & -1.5277445 \\ \mathrm{H} & 2.2683961 & -2.4997642 & 2.1253492 \\ \mathrm{H} & 2.5036711 & -1.4731175 & 4.3822604 \\ \mathrm{H} & 1.1805773 & 0.5633526 & 4.9679603 \\ \mathrm{H} & -0.3757705 & 1.5591851 & 3.3333658 \\ \mathrm{H} & -3.1899036 & 0.8426347 & -0.7275487 \\ \mathrm{H} & -3.9938211 & -0.7869520 & -2.3789794 \\ \mathrm{H} & -2.6575389 & -2.8308146 & -2.9080896 \\ \mathrm{H} & -0.4829372 & -3.2227250 & -1.7619398 \\ \mathrm{O} & -2.6000988 & 1.8462648 & 1.9825881 \\ \mathrm{H} & -3.2496932 & 1.2073616 & 1.6352905 \\ \mathrm{H} & -1.9189382 & 2.3852567 & 0.0734945 \\ \mathrm{H} & -0.8302606 & 2.6899425 & 1.4382491 \\ \mathrm{H} & 2.2773401 & -1.6100211 & -2.2096950 \\ \mathrm{O} & 2.7914291 & 0.1639888 & -3.0773992 \\ \mathrm{H} & 0.8826379 & -0.6454488 & -2.7409955 \\ \mathrm{C} & 0.3663385 & 1.9539866 & -1.7185662 \\ \mathrm{O} & 1.2924178 & 2.4819386 & -2.7327188 \\ \mathrm{H} & -0.3134504 & 1.3015295 & -2.2827150 \\ \mathrm{H} & -0.2171395 & 2.7932188 & -1.3162076 \\ \mathrm{H} & 2.4117937 & 1.0789746 & -3.1076729 \\ \mathrm{H} & 1.8305520 & 3.1719607 & -2.3002993\end{array}$

\section{Product $3+\mathrm{A}$}

Imaginary Frequencies $=0$

Energy (BP86/def-TZVP, level of optimization) $=-962.1116414665 \mathrm{H}$ Energy (M06-2X/def2-TZVP) $=-961.6942656990 \mathrm{H}$

$\begin{array}{lrrr}\text { C } & 1.5363244 & -0.1510664 & 0.3160887 \\ \text { C } & 2.3194690 & -0.8160994 & -0.8201293 \\ \text { C } & 3.6700009 & -1.0646639 & -0.5065749 \\ \text { C } & 4.0564288 & -0.6265226 & 0.8883209 \\ \text { C } & 0.0823495 & 0.1846139 & -0.0586159 \\ \text { H } & 5.1158107 & -0.8058148 & 1.1042757 \\ \mathrm{C} & 1.6388018 & -1.1681850 & 1.5437684 \\ \mathrm{C} & 3.1531595 & -1.4444785 & 1.8803039 \\ \mathrm{H} & 1.2127516 & -2.1184239 & 1.1884193 \\ \mathrm{H} & 3.3551763 & -2.5091605 & 1.6864794 \\ \mathrm{C} & 3.5595287 & -1.1841346 & 3.3321116 \\ \mathrm{C} & 2.3299091 & 1.0962792 & 0.7185423 \\ \mathrm{C} & 3.6799658 & 0.8301868 & 1.0281903 \\ \mathrm{C} & 4.5173057 & -1.6706927 & -1.4369345 \\ \mathrm{C} & 4.0175206 & -2.0275787 & -2.6980321 \\ \mathrm{C} & 2.6760830 & -1.7828367 & -3.0136135 \\ \mathrm{C} & 1.8227455 & -1.1812942 & -2.0755974 \\ \mathrm{C} & 1.8518734 & 2.4096228 & 0.7998791 \\ \mathrm{C} & 2.7165116 & 3.4453303 & 1.1867955\end{array}$




$\begin{array}{rrrr}\mathrm{C} & 4.0522270 & 3.1739277 & 1.5001616 \\ \mathrm{C} & 4.5360067 & 1.8599087 & 1.4236735 \\ \mathrm{H} & 5.5636604 & -1.8586657 & -1.1839279 \\ \mathrm{H} & 4.6760989 & -2.4948327 & -3.4328818 \\ \mathrm{H} & 2.2876971 & -2.0616737 & -3.9952109 \\ \mathrm{H} & 0.7755855 & -1.0132619 & -2.3289283 \\ \mathrm{H} & 0.8147332 & 2.6477358 & 0.5603298 \\ \mathrm{H} & 2.3400193 & 4.4686524 & 1.2413653 \\ \mathrm{H} & 4.7193857 & 3.9840479 & 1.8011491 \\ \mathrm{H} & 5.5787565 & 1.6405075 & 1.6657923 \\ \mathrm{O} & -0.7559414 & -0.9638006 & -0.2430275 \\ \mathrm{H} & -0.9315613 & -1.2736983 & 0.6788358 \\ \mathrm{H} & 0.0720231 & 0.7473393 & -1.0037631 \\ \mathrm{H} & -0.3529267 & 0.8394652 & 0.7142247 \\ \mathrm{C} & 0.7617893 & -0.7437245 & 2.7293417 \\ \mathrm{H} & 1.1096022 & -1.2021850 & 3.6654576 \\ \mathrm{H} & 0.7628019 & 0.3506570 & 2.8601418 \\ \mathrm{O} & -0.5924377 & -1.2131468 & 2.4769154 \\ \mathrm{H} & 3.3759731 & -0.1300625 & 3.6077782 \\ \mathrm{O} & 4.9606748 & -1.5003453 & 3.4657850 \\ \mathrm{H} & 2.9739142 & -1.8268799 & 4.0096352 \\ \mathrm{H} & -1.1959678 & -0.7369697 & 3.0753613 \\ \mathrm{H} & 5.2046775 & -1.3331009 & 4.3935180\end{array}$

TS $4+\mathrm{A}$ (endo)

Imaginary Frequencies $=1(-386.39 \mathrm{~cm}-1)$

Energy (BP86/def-TZVP, level of optimization) $=-691.8254476344 \mathrm{H}$ Energy (M06-2X/def2-TZVP) $=-691.4790312935 \mathrm{H}$

$\begin{array}{lrrr}\mathrm{C} & 1.4403042 & 0.1805351 & 0.8996331 \\ \mathrm{C} & 0.8151138 & -0.9222925 & 1.4870077 \\ \mathrm{C} & -0.4648254 & -0.8357304 & 2.0342741 \\ \mathrm{C} & -1.2071070 & 0.3588261 & 1.9931966 \\ \mathrm{H} & 1.2847489 & -1.9030206 & 1.3755623 \\ \mathrm{H} & -0.9652303 & -1.7560743 & 2.3487619 \\ \mathrm{C} & -0.1959505 & 0.7892980 & -0.7346951 \\ \mathrm{C} & -1.4505575 & 0.6972464 & -0.0987156 \\ \mathrm{C} & 0.2678458 & -0.2143975 & -1.7568773 \\ \mathrm{C} & -2.4255457 & -0.4088131 & -0.4622834 \\ \mathrm{O} & -3.0298049 & -0.1973677 & -1.7594107 \\ \mathrm{O} & -0.5389416 & -0.1663416 & -2.9838126 \\ \mathrm{C} & 2.7318105 & 0.0268687 & 0.2210085 \\ \mathrm{H} & 1.2091525 & 1.1908102 & 1.2303242 \\ \mathrm{H} & -0.6415066 & 1.2923382 & 2.0264961 \\ \mathrm{C} & -2.5709756 & 0.4519447 & 2.6227690 \\ \mathrm{H} & -3.1895170 & 1.2286666 & 2.1504919 \\ \mathrm{H} & -3.1115914 & -0.5035629 & 2.5867435 \\ \mathrm{H} & -2.4611262 & 0.7353405 & 3.6830373 \\ \mathrm{O} & 3.2831816 & -1.0343918 & -0.0726897 \\ \mathrm{O} & 3.2930802 & 1.2367614 & -0.0834674 \\ \mathrm{H} & 4.1398582 & 1.0609170 & -0.5471541 \\ \mathrm{H} & 0.2599678 & 1.7796592 & -0.8023802 \\ \mathrm{H} & -1.9204918 & 1.6549490 & 0.1462792 \\ \mathrm{H} & 0.1590296 & -1.2454141 & -1.3969463\end{array}$




$\begin{array}{lrrr}\mathrm{H} & 1.3194737 & -0.0500486 & -2.0279426 \\ \mathrm{H} & -3.2594601 & -0.4366164 & 0.2494097 \\ \mathrm{H} & -1.9232930 & -1.3909920 & -0.4297540 \\ \mathrm{H} & -2.2697229 & -0.1719685 & -2.3944558 \\ \mathrm{H} & -0.3641064 & 0.6920388 & -3.4145479\end{array}$

TS $4+A$ (exo)

Imaginary Frequencies $=1(-431.33 \mathrm{~cm}-1)$

Energy (BP86/def-TZVP, level of optimization) $=-691.8219487779 \mathrm{H}$ Energy (M06-2X/def2-TZVP) $=-691.4831496906 \mathrm{H}$

C $\quad-0.4326326-0.0489847 \quad-2.0607832$

C $\quad 0.9412915 \quad-0.0532453 \quad-2.2862249$

C $\quad 1.8782219 \quad 0.3524673 \quad-1.3178026$

$\mathrm{H} \quad-1.0905043 \quad-0.5123903 \quad-2.7988991$

$\mathrm{H} \quad 1.3139419 \quad-0.5399185 \quad-3.1925780$

C $\quad 0.1649806 \quad-1.2204766 \quad 0.5654172$

C $\quad \begin{array}{lll}1.5272983 & -0.9050484 & 0.4179684\end{array}$

$\begin{array}{llll}\mathrm{H} & -0.2201107 & -2.0280497 & -0.0605998\end{array}$

$\begin{array}{llll}\text { C } & -0.6144750 & -1.0408126 & 1.8582638\end{array}$

2.2599638

$-1.6453094$

$-0.1083985$

1.7420162

$-0.1372656$

1.5052464

$-0.7697734$

1.1783241

1.7683481

$-2.3807727$

0.3202666

2.3350565

$-0.4215337$

0.2576538

$-0.4887436$

1.5974649

1.0364458

$-0.1952469$

3. 3489542

1.1598741

$-0.6347534$

0.2593277

$-1.6263180$

3. 9644508

0.1787728

$-0.7202656$

3.5729167

$-0.5960530$

$-2.2791480$

3. 6767747

1.1757741

$-2.1457148$

$-2.8606919$

0.6990004

0.5712852

$-3.1661357$

$-0.3586515$

$-1.4062617$

$-4.0825683$

$-0.3670994$

$-1.0557381$

$-0.0889770$

$-1.5768344$

2. 6670073

$-1.6065066$

$-1.5026975$

1.7515703

3.3159100

$-0.0082497$

1.2340803

2. 2441571

$-0.7437655$

2. 4341561

0.7960173

1.0497683

2.0487756

$-1.5156229$

0.7017392

1. 8010862

\section{Product $4+A$ (endo)}

Imaginary Frequencies $=0$

Energy (BP86/def-TZVP, level of optimization) $=-691.8880669589 \mathrm{H}$

Energy (M06-2X/def2-TZVP) $=-691.5646471295 \mathrm{H}$

$\begin{array}{lrrr}\text { C } & 1.4599828 & 0.0135665 & 0.3479037 \\ \text { C } & 2.1884604 & 0.4178883 & -0.9104194 \\ \text { C } & 3.4626498 & 0.0792961 & -1.1511816 \\ \text { C } & 4.3292233 & -0.7479466 & -0.2324946 \\ \text { H } & 1.6379209 & 1.0285489 & -1.6297237 \\ \text { H } & 3.9345608 & 0.4170605 & -2.0800062 \\ \text { C } & 2.0686107 & -1.2810943 & 0.9753709 \\ \text { C } & 3.5981790 & -1.0170922 & 1.1159350\end{array}$




$\begin{array}{rrrr}\mathrm{C} & 1.6317286 & -2.5302750 & 0.1827248 \\ \mathrm{C} & 4.3423295 & -1.9321348 & 2.0890489 \\ \mathrm{O} & 4.3853961 & -3.3139229 & 1.6438493 \\ \mathrm{O} & 1.8680708 & -3.7653598 & 0.8692820 \\ \mathrm{C} & -0.0434040 & -0.0861172 & 0.1424516 \\ \mathrm{H} & 1.5813909 & 0.8116922 & 1.1045753 \\ \mathrm{H} & 5.2015575 & -0.1168868 & 0.0243972 \\ \mathrm{C} & 4.9124837 & -1.9619153 & -0.9915450 \\ \mathrm{H} & 5.6499896 & -2.4956086 & -0.3797845 \\ \mathrm{H} & 4.1382025 & -2.6815337 & -1.2892703 \\ \mathrm{H} & 5.4139974 & -1.6109424 & -1.9066323 \\ \mathrm{O} & -0.6346035 & 0.0033854 & -0.9243723 \\ \mathrm{O} & -0.6917394 & -0.2750011 & 1.3206657 \\ \mathrm{H} & -1.6530254 & -0.3397777 & 1.1306686 \\ \mathrm{H} & 1.6533273 & -1.3876098 & 1.9910399 \\ \mathrm{H} & 3.6428036 & -0.0497340 & 1.6499497 \\ \mathrm{H} & 2.1003961 & -2.5360458 & -0.8161005 \\ \mathrm{H} & 0.5429694 & -2.4904381 & 0.0236633 \\ \mathrm{H} & 3.8487754 & -1.8809779 & 3.0738782 \\ \mathrm{H} & 5.3756201 & -1.5628042 & 2.2040632 \\ \mathrm{H} & 4.7538703 & -3.8488189 & 2.3706070 \\ \mathrm{H} & 2.8216831 & -3.7541680 & 1.1582740\end{array}$

\section{Product $4+A$ (exo)}

Imaginary Frequencies $=0$

Energy (BP86/def-TZVP, level of optimization) $=-691.8964066156 \mathrm{H}$ Energy (M06-2X/def2-TZVP) $=-691.5745413629 \mathrm{H}$

$\begin{array}{lrrr}\text { C } & 1.1592939 & 0.2607554 & 0.3613624 \\ \text { C } & 2.1123046 & 0.8843011 & -0.6426410 \\ \text { C } & 3.4422898 & 0.8887308 & -0.4754891 \\ \text { C } & 4.1733877 & 0.2333668 & 0.6696594 \\ \text { H } & 1.6644031 & 1.3621741 & -1.5167009 \\ \text { H } & 4.0636030 & 1.3965350 & -1.2214688 \\ \text { C } & 1.8455721 & -0.0151238 & 1.7170238 \\ \text { C } & 3.2282173 & -0.6834995 & 1.4750126 \\ \text { H } & 1.2163427 & -0.7309868 & 2.2729628 \\ \text { C } & 1.8605050 & 1.2990215 & 2.5209191 \\ \text { H } & 3.0141714 & -1.5634092 & 0.8394025 \\ \text { C } & 3.8790363 & -1.2787682 & 2.7375021 \\ \text { O } & 4.4848291 & -0.3259240 & 3.6251432 \\ \text { O } & 2.2283933 & 1.1357609 & 3.9138847 \\ \text { C } & 0.4829913 & -0.9788379 & -0.2246818 \\ \text { H } & 0.3278357 & 0.9734568 & 0.5151800 \\ \text { H } & 4.5386416 & 1.0344339 & 1.3410347 \\ \text { C } & 5.4178763 & -0.5122151 & 0.1495661 \\ \text { H } & 6.0387416 & -0.8838680 & 0.9763347 \\ \text { H } & 5.1275690 & -1.3702601 & -0.4767117 \\ \text { H } & 6.0418210 & 0.1574483 & -0.4608060 \\ \text { O } & 0.4904038 & -2.1005789 & 0.2586364 \\ \text { O } & -0.1670173 & -0.6861053 & -1.3789315 \\ \text { H } & -0.5826527 & -1.5114921 & -1.7113624 \\ \text { H } & 0.8631582 & 1.7644409 & 2.4709025 \\ \text { H } & 2.5864284 & 2.0150674 & 2.1130531\end{array}$




$\begin{array}{rrrr}\mathrm{H} & 4.6829721 & -1.9671902 & 2.4415721 \\ \mathrm{H} & 3.1193396 & -1.8796565 & 3.2737913 \\ \mathrm{H} & 3.7466936 & 0.2733530 & 3.9095926 \\ \mathrm{H} & 1.5254484 & 0.6199274 & 4.3516975\end{array}$

TS $5+A$ (endo)

Imaginary Frequencies $=1(-410.41 \mathrm{~cm}-1)$

Energy (BP86/def-TZVP, level of optimization) $=-1032.7747000926 \mathrm{H}$ Energy (M0 6-2X/def2-TZVP) $=-1032.269236219 \mathrm{H}$

$\begin{array}{llll}\text { C } & -1.0877678 & -0.0196961 & 0.2391720\end{array}$

C $\quad-0.6822662 \quad 0.0315857 \quad-1.1288219$

$\begin{array}{llll}\text { C } & 0.6973023 & 0.0258969 & -1.1304242\end{array}$

C $\quad 1.1049241 \quad-0.0210465 \quad 0.2347424$

$\begin{array}{lrrr}0 & 0.0130168 & 0.3453121 & 1.0111747\end{array}$

$\mathrm{H} \quad-1.3491060 \quad-0.0446777 \quad-1.9836579$

$\mathrm{H} \quad 1.3619771 \quad-0.0568788-1.9863908$

$\begin{array}{llll}\text { C } & -2.4269886 & 0.3395797 & 0.8212221\end{array}$

$\begin{array}{llll}\mathrm{H} & -2.4600811 & 0.0492985 & 1.8808054\end{array}$

$\begin{array}{llll}\mathrm{H} & -3.2124138 & -0.2099292 & 0.2867849\end{array}$

$\begin{array}{llll}\text { C } & 2.4521448 & 0.3005597 & 0.8176185\end{array}$

$\mathrm{H} \quad 2.4802202 \quad 0.0014542 \quad 1.8747269$

$\mathrm{H} \quad 3.2237059 \quad-0.2623660 \quad 0.2771097$

$\begin{array}{llll}\mathrm{N} & -2.7488427 & 1.7611402 & 0.7063677\end{array}$

$\begin{array}{lrrr}\mathrm{N} & 2.8055872 & 1.7159334 & 0.7137015\end{array}$

$\begin{array}{llll}\text { C } & -3.6715128 & 2.2530891 & -0.1646949\end{array}$

$\begin{array}{llll}\text { C } & 3.7307806 & 2.1946336 & -0.1618351\end{array}$

$\begin{array}{llll}\text { C } & 3.9508861 & 3.6931467 & -0.1311913\end{array}$

$\begin{array}{llll}\mathrm{H} & 3.7405954 & 4.1019412 & -1.1294809\end{array}$

$\begin{array}{llll}\mathrm{H} & 3.3237884 & 4.2097177 & 0.6061808\end{array}$

$\begin{array}{llll}\mathrm{H} & 5.0078710 & 3.8930190 & 0.0932822\end{array}$

$\begin{array}{llll}0 & 4.3650774 & 1.4542970 & -0.9363828\end{array}$

$\begin{array}{llll}\mathrm{H} & 2.3174860 & 2.3750100 & 1.3145374\end{array}$

$\begin{array}{llll}\text { C } & -3.8575990 & 3.7564027 & -0.1444752\end{array}$

$\begin{array}{llll}\mathrm{H} & -3.2135103 & 4.2644753 & 0.5841386\end{array}$

$\mathrm{H} \quad-3.6456128 \quad 4.1526146 \quad-1.1474692$

$\begin{array}{llll}\mathrm{H} & -4.9080868 & 3.9817208 & 0.0862309\end{array}$

$\begin{array}{llll}0 & -4.3289058 & 1.5209702 & -0.9275358\end{array}$

$\mathrm{H} \quad-2.2415308 \quad 2.4143174 \quad 1.2977755$

$\begin{array}{llll}\text { C } & 0.6792593 & -2.1195568 & 0.6471884\end{array}$

$\begin{array}{llll}\text { C } & -0.7396043 & -2.0841961 & 0.6483368\end{array}$

$\mathrm{H} \quad 1.1689942 \quad-2.0520270 \quad 1.6236322$

$\begin{array}{llll}\text { C } & 1.4518365 & -2.8991580 & -0.3837281\end{array}$

$\mathrm{H} \quad-1.2147948 \quad-2.0122035 \quad 1.6319277$

C $\quad-1.5484623$

C -1.5484623

$-2.8544954-0.3718685$

$\begin{array}{lll}-1.5304594 & -4.2837553 & -0.1205077\end{array}$

$\begin{array}{lll}1.2901680 & -4.3523978 & -0.2316852\end{array}$

$\begin{array}{lll}2.5250790 & -2.6593679 & -0.3406168\end{array}$

$\begin{array}{lll}1.0944342 & -2.6936286 & -1.4004542\end{array}$

$\begin{array}{lll}-1.1841316 & -2.6499490 & -1.3934226\end{array}$

$\begin{array}{lll}-2.6067532 & -2.5614386 & -0.3317547\end{array}$

$\begin{array}{lll}-0.5715915 & -4.5304103 & -0.1260376\end{array}$

$\begin{array}{llll}\mathrm{H} & 1.6666182 & -4.5966636 & 0.6350798\end{array}$ 


\section{TS $5+\mathrm{A}$ (exo)}

Imaginary Frequencies $=1(-409.99 \mathrm{~cm}-1)$

Energy (BP86/def-TZVP, level of optimization) $=-1032.7706085244 \mathrm{H}$ Energy (M06-2X/def2-TZVP) $=-1032.265407465 \mathrm{H}$$$
\text { C } \quad-0.0626618
$$$$
\text { C } \quad 0.4592286
$$$$
\text { C } \quad 0.4256970
$$$$
\text { C } \quad-0.1078463
$$$$
\text { o } \quad-0.0205289
$$$$
\mathrm{H} \quad 0.6898642
$$$$
\mathrm{H} \quad 0.6247633
$$$$
\text { C } \quad 0.1663715
$$$$
\mathrm{H} \quad-0.4382878
$$$$
\mathrm{H} \quad-0.1297252
$$$$
\text { C } \quad 0.0331865
$$$$
\mathrm{H} \quad-0.5656357
$$$$
\mathrm{H} \quad-0.3250974
$$$$
\mathrm{N} \quad 1.5679857
$$$$
\mathrm{N} \quad 1.4201846
$$$$
\text { C } \quad 2.3928565
$$$$
\text { C } \quad 2.1618399
$$$$
\text { C } \quad 3.5830011
$$$$
\mathrm{H} \quad 4.2749536
$$$$
\mathrm{H} \quad 3.8328520
$$$$
\mathrm{H} \quad 3.7278277
$$$$
\text { o } \quad 1.7082641
$$$$
\mathrm{H} \quad 1.8554840
$$$$
\text { C } \quad 3.8147624
$$$$
\mathrm{H} \quad 3.9946360
$$$$
\mathrm{H} \quad 4.5018586
$$$$
\mathrm{H} \quad 4.0406898
$$$$
\text { o } \quad 2.0103801
$$$$
\mathrm{H} \quad 1.9467292
$$$$
\text { C } \quad-2.1412393
$$$$
\text { C } \quad-2.1999536
$$$$
\mathrm{H} \quad-2.1525261
$$$$
\text { C } \quad-2.8275000
$$$$
\mathrm{H} \quad-2.2336691
$$$$
\text { C } \quad-2.9149029
$$$$
\text { O } \quad-4.3722972
$$$$
\text { o } \quad-4.2720258
$$$$
\mathrm{H} \quad-2.5482180
$$$$
\mathrm{H} \quad-2.5364658
$$$$
\mathrm{H} \quad-2.6143241
$$$$
\mathrm{H} \quad-2.7234133
$$$$
\mathrm{H} \quad-4.7015414
$$$$
\mathrm{H} \quad-4.5198043
$$$$
-1.1033143
$$$$
-0.6781996
$$$$
0.6956132
$$$$
1.0889928
$$$$
-0.0055636
$$$$
-1.3405000
$$$$
1.3718946
$$$$
-2.4376653
$$$$
-2.5216914
$$$$
-3.2354869
$$$$
2.4339673
$$$$
2.4730399
$$$$
3.2122484
$$$$
-2.6691737
$$$$
2.7624384
$$$$
-3.4908906
$$$$
3.6732215
$$$$
3.8649449
$$$$
3.6313330
$$$$
\text { 3. } 2395487
$$$$
\text { 4. } 9213222
$$$$
4.3158636
$$$$
2.2644002
$$$$
-3.5905712
$$$$
-2.9898612
$$$$
-3.2616425
$$$$
-4.6434254
$$$$
-4.1284027
$$$$
-2.1697573
$$$$
-0.7970433
$$$$
0.6257669
$$$$
-1.2851143
$$$$
-1.6055031
$$$$
1.1153971
$$$$
1.3872283
$$$$
1.1836870
$$$$
-1.5866338
$$$$
-2.6646893
$$$$
-1.2467432
$$$$
1.0535923
$$$$
2.4666570
$$$$
1.5573230
$$$$
0.2183310
$$$$
\text { 1. } 4824107
$$$$
1.4797647
$$$$
0.2124824
$$$$
-0.6364803
$$$$
\text { 2. } 3128991
$$$$
\text { 2. } 3072650
$$$$
-0.4392453
$$$$
-1.3513495
$$$$
0.2535225
$$$$
-0.4462981
$$$$
-1.3655534
$$$$
0.2393703
$$$$
-0.7905402
$$$$
-0.7794514
$$$$
-0.0865848
$$$$
-0.0921853
$$$$
-0.5816442
$$$$
0.2396704
$$$$
-1.4480534
$$$$
-0.8474986
$$$$
0.8727213
$$$$
-1.5512419
$$$$
-0.6007228
$$$$
-1.5012557
$$$$
0.1915819
$$$$
-0.8199625
$$$$
0.9119681
$$$$
-1.5907680
$$$$
0.6656159
$$$$
0.6698565
$$$$
1.6429646
$$$$
-0.4171232
$$$$
\text { 1. } 6459103
$$$$
-0.4173579
$$$$
-0.3918921
$$$$
-0.2858326
$$$$
-0.3457219
$$$$
-1.4206583
$$$$
-1.4190007
$$$$
-0.3374146
$$$$
0.4475941
$$$$
-0.6268112-0.3113479
$$

\section{Product $5+\mathrm{A}$ (endo)}

Imaginary Frequencies $=0$

Energy(BP86/def-TZVP, level of optimization) $=-1032.8147040112 \mathrm{H}$ Energy (M0 6-2X/def2-TZVP) $=-1032.327238731 \mathrm{H}$
C
0.5086976
3.8272342
0.3376270 


$\begin{array}{rrrr}\text { C } & 0.9530705 & 4.1503766 & -1.0863111 \\ \mathrm{C} & 2.2924435 & 4.0948275 & -1.0761288 \\ \mathrm{C} & 2.6852499 & 3.7355949 & 0.3547425 \\ \mathrm{O} & 1.6173862 & 4.3729308 & 1.1160420 \\ \mathrm{H} & 0.2864837 & 4.2894317 & -1.9357219 \\ \mathrm{H} & 2.9816278 & 4.1776862 & -1.9149713 \\ \mathrm{C} & -0.8172509 & 4.3751478 & 0.8326569 \\ \mathrm{H} & -0.9459133 & 4.1039231 & 1.8927086 \\ \mathrm{H} & -1.6441185 & 3.9290624 & 0.2642763 \\ \mathrm{C} & 4.0469772 & 4.1676406 & 0.8701839 \\ \mathrm{H} & 4.1287090 & 3.9047922 & 1.9366881 \\ \mathrm{H} & 4.8398685 & 3.6376200 & 0.3258499 \\ \mathrm{~N} & -0.9143160 & 5.8189350 & 0.6723794 \\ \mathrm{~N} & 4.2842760 & 5.5926016 & 0.6916048 \\ \mathrm{C} & -1.8783728 & 6.4383856 & -0.0603838 \\ \mathrm{C} & 5.2678497 & 6.1028706 & -0.0979467 \\ \mathrm{C} & 5.3387153 & 7.6146696 & -0.1634492 \\ \mathrm{H} & 5.2266808 & 7.9297211 & -1.2103029 \\ \mathrm{H} & 4.5722882 & 8.1123436 & 0.4439115 \\ \mathrm{H} & 6.3322365 & 7.9391817 & 0.1758744 \\ \mathrm{O} & 6.0663563 & 5.3820228 & -0.7255948 \\ \mathrm{H} & 3.6609092 & 6.2382582 & 1.1692200 \\ \mathrm{C} & -1.7968211 & 7.9504429 & -0.1077698 \\ \mathrm{H} & -0.9552269 & 8.3586735 & 0.4659737 \\ \mathrm{H} & -1.7036310 & 8.2677143 & -1.1557957 \\ \mathrm{H} & -2.7344941 & 8.3693009 & 0.2832853 \\ \mathrm{O} & -2.7767140 & 5.8127927 & -0.6545572 \\ \mathrm{H} & -0.2106148 & 6.3909778 & 1.1318619 \\ \mathrm{C} & 2.3154293 & 2.2160798 & 0.5778460 \\ \mathrm{C} & 0.7407180 & 2.2825052 & 0.5671047 \\ \mathrm{H} & 2.6542207 & 1.9475632 & 1.5901214 \\ \mathrm{C} & 3.0013161 & 1.2742430 & -0.4018198 \\ \mathrm{H} & 0.3732394 & 2.0482946 & 1.5783000 \\ \mathrm{C} & -0.0127645 & 1.3806269 & -0.4146927 \\ \mathrm{O} & 0.0043873 & -0.0041277 & -0.0261797 \\ \mathrm{O} & 2.7582698 & -0.0920893 & 0.0292779 \\ \mathrm{H} & 4.0878105 & 1.4626399 & -0.4017527 \\ \mathrm{H} & 2.6304970 & 1.4154394 & -1.4296440 \\ \mathrm{H} & 0.3796744 & 1.5008472 & -1.4404551 \\ \mathrm{H} & -1.0729716 & 1.6741983 & -0.4369707 \\ \mathrm{H} & 0.9624560 & -0.2559169 & 0.0115347 \\ \mathrm{H} & 3.1877480 & -0.6868170 & -0.6133057 \\ & & & \end{array}$

\section{Product $5+$ A (exo)}

Imaginary Frequencies $=0$

Energy (BP86/def-TZVP, level of optimization) $=-1032.8108890958 \mathrm{H}$

Energy (M0 6-2X/def2-TZVP) $=-1032.323149839 \mathrm{H}$

$\begin{array}{llrr}\text { C } & 4.0359784 & -2.1277427 & 0.2759053 \\ \text { C } & 4.7886663 & -1.6537290 & 1.5180643 \\ \text { C } & 4.6918799 & -0.3193969 & 1.5182874 \\ \text { C } & 3.8779562 & 0.0436894 & 0.2780257 \\ \text { O } & 4.2550879 & -1.0205609 & -0.6462624 \\ \text { H } & 5.1954923 & -2.3119310 & 2.2839464\end{array}$




$\begin{array}{rrrr}\mathrm{H} & 4.9996946 & 0.3892379 & 2.2854563 \\ \mathrm{C} & 4.4843228 & -3.4416450 & -0.3440070 \\ \mathrm{H} & 3.9629909 & -3.6079821 & -1.2986316 \\ \mathrm{H} & 4.2436193 & -4.2739313 & 0.3305355 \\ \mathrm{C} & 4.1289241 & 1.4084027 & -0.3413410 \\ \mathrm{H} & 3.5822014 & 1.4984750 & -1.2913452 \\ \mathrm{H} & 3.7748247 & 2.1962963 & 0.3367534 \\ \mathrm{~N} & 5.9230084 & -3.4707231 & -0.5692761 \\ \mathrm{~N} & 5.5469697 & 1.6463329 & -0.5770411 \\ \mathrm{C} & 6.7638947 & -4.3649349 & 0.0166453 \\ \mathrm{C} & 6.2553341 & 2.6507141 & 0.0050568 \\ \mathrm{C} & 7.7217968 & 2.7215735 & -0.3694199 \\ \mathrm{H} & 8.3259465 & 2.6158464 & 0.5425124 \\ \mathrm{H} & 8.0240913 & 1.9514837 & -1.0902262 \\ \mathrm{H} & 7.9327878 & 3.7132274 & -0.7932264 \\ \mathrm{O} & 5.7416507 & 3.4664107 & 0.7939659 \\ \mathrm{H} & 6.0313857 & 1.0140770 & -1.2086806 \\ \mathrm{C} & 8.2288341 & -4.2203112 & -0.3420538 \\ \mathrm{H} & 8.4248991 & -3.4039071 & -1.0485289 \\ \mathrm{H} & 8.8033495 & -4.0437436 & 0.5780956 \\ \mathrm{H} & 8.5830722 & -5.1649655 & -0.7774147 \\ \mathrm{O} & 6.3652470 & -5.2509270 & 0.7961133 \\ \mathrm{H} & 6.3158718 & -2.7689631 & -1.1911717 \\ \mathrm{C} & 2.4995392 & -1.9406567 & 0.6136236 \\ \mathrm{C} & 2.3847354 & -0.3607857 & 0.6197469 \\ \mathrm{H} & 2.2999868 & -2.3406900 & 1.6185752 \\ \mathrm{C} & 1.6044639 & -2.7042683 & -0.3578415 \\ \mathrm{H} & 2.1394729 & -0.0086753 & 1.6324133 \\ \mathrm{C} & 1.3566701 & 0.2755744 & -0.3275931 \\ \mathrm{O} & 0.0001157 & 0.0884975 & 0.1078087 \\ \mathrm{O} & 0.2272739 & -2.6065149 & 0.0962809 \\ \mathrm{H} & 1.8909847 & -3.7669608 & -0.3613824 \\ \mathrm{H} & 1.6936496 & -2.3183287 & -1.3863646 \\ \mathrm{H} & 1.4916282 & -0.0886234 & -1.3625339 \\ \mathrm{H} & 1.5104201 & 1.3635312 & -0.3409316 \\ \mathrm{H} & -0.1380892 & -0.8943944 & 0.1269671 \\ \mathrm{H} & -0.3281672 & -3.1008584 & -0.5347832 \\ & & & \end{array}$

\section{TS $1+B$}

Imaginary Frequencies $=1(-373.70 \mathrm{~cm}-1)$

Energy (BP86/def-TZVP, level of optimization) $=-1319.0877684878 \mathrm{H}$ Energy (M06-2X/def2-TZVP) $=-1318.515412320 \mathrm{H}$

$\begin{array}{lrrr}\text { C } & -0.9739532 & 0.1614706 & -1.7850458 \\ \mathrm{C} & -0.0586722 & 0.0084124 & -2.9037767 \\ \mathrm{C} & 1.3018434 & 0.3747546 & -2.6820154 \\ \mathrm{C} & 1.6529103 & 0.8233475 & -1.3585878 \\ \mathrm{C} & -2.3595755 & -0.4083203 & -1.9152026 \\ \mathrm{H} & 2.7022781 & 1.0601642 & -1.1675743 \\ \mathrm{C} & 0.2145315 & -1.5570232 & -0.5280819 \\ \mathrm{C} & 1.5265920 & -1.0659018 & -0.4157062 \\ \mathrm{H} & -0.1530984 & -2.2576209 & -1.2699406 \\ \mathrm{C} & -0.4419142 & -1.4603689 & 0.7887340 \\ \mathrm{H} & 2.3710573 & -1.4526286 & -0.9820705\end{array}$




\begin{tabular}{lrrr}
$\mathrm{C}$ & 1.7492975 & -0.7047498 & 1.0201322 \\
$\mathrm{O}$ & 2.7849717 & -0.3247278 & 1.5508144 \\
$\mathrm{O}$ & -1.5643568 & -1.8212651 & 1.1251545 \\
$\mathrm{~N}$ & 0.5129955 & -0.8801310 & 1.6781277 \\
$\mathrm{C}$ & 0.2822526 & -0.6427446 & 3.0670532 \\
$\mathrm{C}$ & 1.1454715 & -1.2010183 & 4.0211254 \\
$\mathrm{C}$ & 0.9102251 & -0.9717390 & 5.3798741 \\
$\mathrm{C}$ & -0.1875675 & -0.2046598 & 5.7879305 \\
$\mathrm{C}$ & -1.0503141 & 0.3407765 & 4.8300580 \\
$\mathrm{C}$ & -0.8156939 & 0.1299480 & 3.4681932 \\
$\mathrm{H}$ & 1.9883465 & -1.8135136 & 3.7022230 \\
$\mathrm{H}$ & 1.5834183 & -1.4041560 & 6.1218685 \\
$\mathrm{H}$ & -1.9072839 & 0.9401231 & 5.1418130 \\
$\mathrm{H}$ & -1.4733493 & 0.5670312 & 2.7177348 \\
$\mathrm{H}$ & -0.3708063 & -0.0336375 & 6.8500080 \\
$\mathrm{C}$ & -0.7105912 & 1.2148284 & -0.8218817 \\
$\mathrm{C}$ & 0.6635436 & 1.5608995 & -0.6185553 \\
$\mathrm{C}$ & 2.2583669 & 0.2108546 & -3.7067075 \\
$\mathrm{C}$ & 1.8699682 & -0.2578788 & -4.9534104 \\
$\mathrm{C}$ & 0.5214852 & -0.5996272 & -5.1882332 \\
$\mathrm{C}$ & -0.4255493 & -0.4859660 & -4.1804456 \\
$\mathrm{C}$ & -1.6907572 & 1.8783861 & -0.0378415 \\
$\mathrm{C}$ & -1.3193482 & 2.8692962 & 0.8589992 \\
$\mathrm{C}$ & 0.0368065 & 3.2088629 & 1.0491144 \\
$\mathrm{C}$ & 1.0188373 & 2.5435770 & 0.3333687 \\
$\mathrm{H}$ & 3.2985877 & 0.4794129 & -3.5119481 \\
$\mathrm{H}$ & 2.6042363 & -0.3578209 & -5.7542357 \\
$\mathrm{H}$ & 0.2172431 & -0.9567203 & -6.1734046 \\
$\mathrm{H}$ & -1.4590625 & -0.7622630 & -4.3843449 \\
$\mathrm{H}$ & -2.7412064 & 1.6336590 & -0.1731040 \\
$\mathrm{H}$ & -2.0911797 & 3.3940780 & 1.4248017 \\
$\mathrm{H}$ & 0.3095293 & 3.9916129 & 1.7585923 \\
$\mathrm{H}$ & 2.0736715 & 2.7859563 & 0.4752399 \\
$\mathrm{O}$ & -2.3472853 & -1.7087970 & -2.2907101 \\
$\mathrm{H}$ & -3.2803857 & -2.0010083 & -2.3885341 \\
$\mathrm{O}$ & -3.4012049 & 0.2052234 & -1.7199994 \\
& & & \\
\hline
\end{tabular}

\section{Product $1+B$}

Imaginary Frequencies $=0$

Energy(BP86/def-TZVP, level of optimization) $=-1319.1422829457 \mathrm{H}$

Energy (M0 6-2X/def2-TZVP) $=-1318.587489730 \mathrm{H}$

$\begin{array}{lllr}\text { C } & 1.4752147 & -0.3237204 & 0.2397664 \\ \text { C } & 2.1626419 & -0.9657944 & -0.9837543 \\ \text { C } & 3.5229088 & -1.2549027 & -0.7682702 \\ \text { C } & 4.0166053 & -0.8830530 & 0.6137507 \\ \text { C } & 0.0049687 & -0.0537408 & -0.0804219 \\ \text { H } & 5.0829798 & -1.0947220 & 0.7547588 \\ \text { C } & 1.6571805 & -1.3569859 & 1.4228016 \\ \text { C } & 3.1418061 & -1.7269688 & 1.6053477 \\ \text { H } & 1.0375807 & -2.2413680 & 1.2286588 \\ \text { C } & 1.2280552 & -0.7627266 & 2.7618705 \\ \text { H } & 3.3329714 & -2.7940947 & 1.4309297 \\ \text { C } & 3.4564029 & -1.4261813 & 3.0625859\end{array}$




$\begin{array}{rrrr}\mathrm{O} & 4.5136883 & -1.6166194 & 3.6425759 \\ \mathrm{O} & 0.1506609 & -0.2592975 & 3.0357592 \\ \mathrm{~N} & 2.3048758 & -0.8792601 & 3.6600640 \\ \mathrm{C} & 2.2399619 & -0.4566019 & 5.0311970 \\ \mathrm{C} & 3.0689737 & 0.5794839 & 5.4747362 \\ \mathrm{C} & 3.0005377 & 0.9853913 & 6.8107794 \\ \mathrm{C} & 2.1045251 & 0.3660952 & 7.6899660 \\ \mathrm{C} & 1.2772524 & -0.6670204 & 7.2343729 \\ \mathrm{C} & 1.3445578 & -1.0868064 & 5.9025368 \\ \mathrm{H} & 3.7566115 & 1.0647156 & 4.7813782 \\ \mathrm{H} & 3.6460607 & 1.7917622 & 7.1621953 \\ \mathrm{H} & 0.5791035 & -1.1534451 & 7.9173302 \\ \mathrm{H} & 0.7112988 & -1.8982491 & 5.5421312 \\ \mathrm{H} & 2.0508095 & 0.6888874 & 8.7309039 \\ \mathrm{C} & 2.3167544 & 0.9022161 & 0.6316217 \\ \mathrm{C} & 3.6751508 & 0.5763249 & 0.8413713 \\ \mathrm{C} & 4.2903218 & -1.8324788 & -1.7804316 \\ \mathrm{C} & 3.6988389 & -2.1127904 & -3.0201524 \\ \mathrm{C} & 2.3482688 & -1.8210436 & -3.2361506 \\ \mathrm{C} & 1.5728747 & -1.2484180 & -2.2172229 \\ \mathrm{C} & 1.8812142 & 2.2143091 & 0.8436209 \\ \mathrm{C} & 2.8027868 & 3.1939382 & 1.2406606 \\ \mathrm{C} & 4.1488071 & 2.8705937 & 1.4376333 \\ \mathrm{C} & 4.5868724 & 1.5542943 & 1.2437160 \\ \mathrm{H} & 5.3448388 & -2.0571320 & -1.6060577 \\ \mathrm{H} & 4.2958606 & -2.5573306 & -3.8182394 \\ \mathrm{H} & 1.8900927 & -2.0374186 & -4.2026251 \\ \mathrm{H} & 0.5201637 & -1.0289011 & -2.4043499 \\ \mathrm{H} & 0.8374326 & 2.4805248 & 0.6904202 \\ \mathrm{H} & 2.4602277 & 4.2184960 & 1.3960627 \\ \mathrm{H} & 4.8587691 & 3.6410941 & 1.7435344 \\ \mathrm{H} & 5.6345504 & 1.2900416 & 1.4024476 \\ \mathrm{O} & -0.7628802 & -1.1503958 & 0.1049659 \\ \mathrm{H} & -1.6794614 & -0.9285411 & -0.1706899 \\ \mathrm{O} & -0.4338574 & 0.9904702 & -0.5348051 \\ & & & \end{array}$

\section{TS $2+B$}

Imaginary Frequencies $=1(-375.68 \mathrm{~cm}-1)$

Energy (BP86/def-TZVP, level of optimization) $=-1130.4270668211 \mathrm{H}$

Energy (M0 6-2X/def2-TZVP) $=-1129.932445497 \mathrm{H}$

$\begin{array}{lrrr}\text { C } & -1.2029978 & 0.7971430 & -1.8332295 \\ \mathrm{C} & -0.6653466 & 0.2501793 & -3.0493178 \\ \mathrm{C} & 0.7094658 & -0.1307057 & -3.0349518 \\ \mathrm{C} & 1.4236501 & 0.0562439 & -1.7987852 \\ \mathrm{H} & -2.2736904 & 1.0124467 & -1.7946006 \\ \mathrm{H} & 2.4531860 & -0.3056517 & -1.7417636 \\ \mathrm{C} & -1.0607330 & -1.2168577 & -0.7515854 \\ \mathrm{C} & 0.2994134 & -1.5786018 & -0.7611678 \\ \mathrm{H} & -1.8249817 & -1.6145686 & -1.4129515 \\ \mathrm{C} & -1.4312755 & -0.8530801 & 0.6345947 \\ \mathrm{H} & 0.7480337 & -2.3131592 & -1.4240705 \\ \mathrm{C} & 0.8177576 & -1.4558516 & 0.6246343 \\ \mathrm{O} & 1.9322396 & -1.7367662 & 1.0520516\end{array}$




$\begin{array}{rrrr}\mathrm{O} & -2.5341544 & -0.5614446 & 1.0853345 \\ \mathrm{~N} & -0.2429569 & -0.9394497 & 1.4123820 \\ \mathrm{C} & -0.1744659 & -0.6879950 & 2.8154401 \\ \mathrm{C} & 0.3377351 & -1.6709801 & 3.6754744 \\ \mathrm{C} & 0.3944814 & -1.4231671 & 5.0500878 \\ \mathrm{C} & -0.0722889 & -0.2101348 & 5.5703467 \\ \mathrm{C} & -0.5921237 & 0.7618174 & 4.7075961 \\ \mathrm{C} & -0.6384337 & 0.5303368 & 3.3297288 \\ \mathrm{H} & 0.6840023 & -2.6217074 & 3.2716261 \\ \mathrm{H} & 0.7963115 & -2.1876264 & 5.7172214 \\ \mathrm{H} & -0.9573606 & 1.7097603 & 5.1062098 \\ \mathrm{H} & -1.0247022 & 1.2908629 & 2.6522239 \\ \mathrm{H} & -0.0320170 & -0.0236104 & 6.6448109 \\ \mathrm{C} & -0.3346922 & 1.5247356 & -0.9536269 \\ \mathrm{C} & 1.0408834 & 1.1380013 & -0.9351416 \\ \mathrm{C} & 1.2713768 & -0.7533610 & -4.1727122 \\ \mathrm{C} & 0.4969092 & -0.9573640 & -5.3071278 \\ \mathrm{C} & -0.8652849 & -0.5808959 & -5.3213649 \\ \mathrm{C} & -1.4461692 & -0.0018181 & -4.2013331 \\ \mathrm{C} & -0.7810403 & 2.5534247 & -0.0888485 \\ \mathrm{C} & 0.1221940 & 3.2033813 & 0.7386222 \\ \mathrm{C} & 1.4813978 & 2.8141748 & 0.7637275 \\ \mathrm{C} & 1.9330863 & 1.7821500 & -0.0449956 \\ \mathrm{H} & 2.3198020 & -1.0581078 & -4.1549095 \\ \mathrm{H} & 0.9397945 & -1.4156036 & -6.1931041 \\ \mathrm{H} & -1.4635710 & -0.7513704 & -6.2180203 \\ \mathrm{H} & -2.5018648 & 0.2770778 & -4.2047918 \\ \mathrm{H} & -1.8326673 & 2.8464154 & -0.1030746 \\ \mathrm{H} & -0.2167676 & 4.0191808 & 1.3795508 \\ \mathrm{H} & 2.1783713 & 3.3322893 & 1.4246364 \\ \mathrm{H} & 2.9815654 & 1.4784522 & -0.0245448 \\ & & & \\ & & & \end{array}$

\section{Product $2+B$}

Imaginary Frequencies $=0$

Energy(BP86/def-TZVP, level of optimization) $=-1130.4834199420 \mathrm{H}$ Energy (M06-2X/def2-TZVP) $=-1130.003756450 \mathrm{H}$

$\begin{array}{lrrr}\text { C } & -1.2404797 & 0.3611875 & -1.5578714 \\ \mathrm{C} & -0.6988014 & 0.0050829 & -2.9329068 \\ \mathrm{C} & 0.6671233 & -0.3417189 & -2.9223381 \\ \mathrm{C} & 1.2924432 & -0.2825345 & -1.5382845 \\ \mathrm{H} & -2.2989860 & 0.6457048 & -1.5772393 \\ \mathrm{H} & 2.3584975 & -0.5381125 & -1.5411139 \\ \mathrm{C} & -1.0238639 & -0.9301417 & -0.6837778 \\ \mathrm{C} & 0.4729832 & -1.3071882 & -0.6693002 \\ \mathrm{H} & -1.6558565 & -1.7370222 & -1.0791124 \\ \mathrm{C} & -1.3985597 & -0.7179907 & 0.7746959 \\ \mathrm{H} & 0.6516457 & -2.3221098 & -1.0506446 \\ \mathrm{C} & 0.8789651 & -1.2798919 & 0.7959113 \\ \mathrm{O} & 1.9854850 & -1.5055385 & 1.2615908 \\ \mathrm{O} & -2.4957172 & -0.4177892 & 1.2204529 \\ \mathrm{~N} & -0.2521962 & -0.9383332 & 1.5629770 \\ \mathrm{C} & -0.2363193 & -0.8253745 & 2.9939925 \\ \mathrm{C} & 0.0592017 & -1.9510815 & 3.7713315\end{array}$




$\begin{array}{rrrr}\text { C } & 0.0808523 & -1.8339037 & 5.1643729 \\ \mathrm{C} & -0.1997437 & -0.6046160 & 5.7724606 \\ \mathrm{C} & -0.4998012 & 0.5136996 & 4.9855897 \\ \mathrm{C} & -0.5144528 & 0.4091291 & 3.5914701 \\ \mathrm{H} & 0.2647406 & -2.9079773 & 3.2904969 \\ \mathrm{H} & 0.3127160 & -2.7080364 & 5.7746729 \\ \mathrm{H} & -0.7182306 & 1.4736000 & 5.4559697 \\ \mathrm{H} & -0.7364543 & 1.2779816 & 2.9713486 \\ \mathrm{H} & -0.1850286 & -0.5180762 & 6.8601169 \\ \mathrm{C} & -0.3482869 & 1.4453662 & -0.9790875 \\ \mathrm{C} & 1.0181155 & 1.0986212 & -0.9693271 \\ \mathrm{C} & 1.3112476 & -0.7056104 & -4.1054338 \\ \mathrm{C} & 0.5860257 & -0.7150716 & -5.3072872 \\ \mathrm{C} & -0.7711279 & -0.3717041 & -5.3176498 \\ \mathrm{C} & -1.4215882 & -0.0133881 & -4.1263266 \\ \mathrm{C} & -0.7645596 & 2.6814990 & -0.4809854 \\ \mathrm{C} & 0.1912219 & 3.5780873 & 0.0200275 \\ \mathrm{C} & 1.5482411 & 3.2342722 & 0.0282775 \\ \mathrm{C} & 1.9671063 & 1.9887203 & -0.4629704 \\ \mathrm{H} & 2.3690145 & -0.9776227 & -4.0966790 \\ \mathrm{H} & 1.0844335 & -0.9920516 & -6.2381120 \\ \mathrm{H} & -1.3280911 & -0.3820207 & -6.2564211 \\ \mathrm{H} & -2.4811802 & 0.2515544 & -4.1338112 \\ \mathrm{H} & -1.8233701 & 2.9498413 & -0.4866513 \\ \mathrm{H} & -0.1265736 & 4.5491659 & 0.4041055 \\ \mathrm{H} & 2.2857467 & 3.9381889 & 0.4182544 \\ \mathrm{H} & 3.0257481 & 1.7198276 & -0.4550184\end{array}$

TS $3+B$

Imaginary Frequencies $=1(-337.28 \mathrm{~cm}-1)$

Energy (BP86/def-TZVP, level of optimization) $=-1245.0083196431 \mathrm{H}$ Energy (M06-2X/def2-TZVP) $=-1244.467140694 \mathrm{H}$

C $\quad-1.1823707-0.1823695-1.8886247$

C $\quad-0.2608171 \quad-0.1377031 \quad-3.0043434$

$\begin{array}{llll}\text { C } & 0.9773760 & 0.5521427 & -2.8275819\end{array}$

C $\quad 1.2429638 \quad 1.1082698 \quad-1.5251050$

C $\quad-2.4015304 \quad-1.0851031 \quad-2.0183131$

$\mathrm{H} \quad 2.2025681 \quad 1.6089912 \quad-1.3736156$

C $\quad 0.4757642-1.5468538 \quad-0.6166606$

C $\quad 1.6427392 \quad-0.7640852 \quad-0.5847525$

$\mathrm{H} \quad 0.2410652 \quad-2.3301451 \quad-1.3300292$

C $\quad-0.1118504 \quad-1.5591884 \quad 0.7239414$

$\mathrm{H} \quad 2.5192235 \quad-0.9411674 \quad-1.2034171$

$\begin{array}{llll}\text { C } & 1.8569560 & -0.3362385 & 0.8313804\end{array}$

$\begin{array}{rrrr}1.8569560 & -0.3362385 & 0.8313804 \\ 0 & 2.7979948 & 0.2852017 & 1.3064017\end{array}$

o $\quad-1.1357996-2.1299360 \quad 1.1206896$

$\mathrm{N} \quad 0.7296327 \quad-0.7846788 \quad 1.5615208$

C $\quad 0.5075331 \quad-0.5487089 \quad 2.9525394$

C $\quad 1.5066760 \quad-0.8803930 \quad 3.8786545$

C $\quad 1.2885334 \quad-0.6471946 \quad 5.2396252$

C $\quad 0.0756584 \quad-0.1021759 \quad 5.6775303$

$\begin{array}{llll}\text { C } & -0.9206053 & 0.2174859 & 4.7474255\end{array}$

$\begin{array}{llll}\text { C } & -0.7063761 & 0.0030143 & 3.3828698\end{array}$ 


$\begin{array}{rrrr}\mathrm{H} & 2.4426083 & -1.3208072 & 3.5358326 \\ \mathrm{H} & 2.0674503 & -0.9021166 & 5.9600958 \\ \mathrm{H} & -1.8673084 & 0.6439255 & 5.0830880 \\ \mathrm{H} & -1.4704889 & 0.2679292 & 2.6523924 \\ \mathrm{H} & -0.0928070 & 0.0727869 & 6.7414406 \\ \mathrm{C} & -1.1314892 & 0.9016453 & -0.9373088 \\ \mathrm{C} & 0.1196788 & 1.5764266 & -0.7605186 \\ \mathrm{C} & 1.9280116 & 0.5807548 & -3.8706239 \\ \mathrm{C} & 1.6483567 & -0.0218841 & -5.0888183 \\ \mathrm{C} & 0.4226657 & -0.6949308 & -5.2777481 \\ \mathrm{C} & -0.5088183 & -0.7682688 & -4.2520266 \\ \mathrm{C} & -2.2511452 & 1.3441903 & -0.1802254 \\ \mathrm{C} & -2.1314846 & 2.4183033 & 0.6880251 \\ \mathrm{C} & -0.8883654 & 3.0627771 & 0.8745022 \\ \mathrm{C} & 0.2267873 & 2.6345863 & 0.1714218 \\ \mathrm{H} & 2.8770564 & 1.0974975 & -3.7121266 \\ \mathrm{H} & 2.3744218 & 0.0246821 & -5.9021432 \\ \mathrm{H} & 0.2038105 & -1.1619960 & -6.2393852 \\ \mathrm{H} & -1.4476698 & -1.2923952 & -4.4289159 \\ \mathrm{H} & -3.2084636 & 0.8410563 & -0.2995028 \\ \mathrm{H} & -3.0086573 & 2.7691268 & 1.2347145 \\ \mathrm{H} & -0.8090268 & 3.9023280 & 1.5669914 \\ \mathrm{H} & 1.1905793 & 3.1307096 & 0.3018817 \\ \mathrm{O} & -3.0619902 & -1.4363921 & -0.8026519 \\ \mathrm{H} & -2.4001442 & -1.8386471 & -0.1887821 \\ \mathrm{H} & -2.1038360 & -1.9965621 & -2.5633015 \\ \mathrm{H} & -3.1560434 & -0.5749812 & -2.6419386\end{array}$

Product $3+B$

Imaginary Frequencies $=0$

Energy (BP86/def-TZVP, level of optimization) $=-1245.0606999641 \mathrm{H}$ Energy (M0 6-2X/def2-TZVP) $=-1244.535418494 \mathrm{H}$

$\begin{array}{lrrr}\text { C } & 1.4778969 & -0.3249156 & 0.2412167 \\ \text { C } & 2.1719112 & -0.9753251 & -0.9663127 \\ \text { C } & 3.5320554 & -1.2729102 & -0.7530798 \\ \text { C } & 4.0239236 & -0.9003835 & 0.6306728 \\ \text { C } & 0.0141935 & 0.0351516 & -0.0026781 \\ \text { H } & 5.0875667 & -1.1217182 & 0.7794916 \\ \text { C } & 1.6494376 & -1.3655602 & 1.4278084 \\ \text { C } & 3.1361018 & -1.7325647 & 1.6207866 \\ \text { H } & 1.0272951 & -2.2416171 & 1.1999817 \\ \mathrm{C} & 1.2021615 & -0.8229561 & 2.7806942 \\ \text { H } & 3.3295164 & -2.8016955 & 1.4592389 \\ \mathrm{C} & 3.4530838 & -1.4140885 & 3.0722125 \\ \mathrm{O} & 4.5179348 & -1.5807002 & 3.6487455 \\ \mathrm{O} & 0.0975916 & -0.4066343 & 3.0938622 \\ \mathrm{~N} & 2.2966625 & -0.8856998 & 3.6693602 \\ \mathrm{C} & 2.2285796 & -0.4674376 & 5.0412330 \\ \mathrm{C} & 3.0201994 & 0.6017758 & 5.4748818 \\ \mathrm{C} & 2.9550683 & 1.0039218 & 6.8122332 \\ \mathrm{C} & 2.0984268 & 0.3474632 & 7.7037020 \\ \mathrm{C} & 1.3077991 & -0.7183922 & 7.2585694 \\ \mathrm{C} & 1.3727928 & -1.1342252 & 5.9252104\end{array}$




$\begin{array}{rrrr}\mathrm{H} & 3.6773864 & 1.1145605 & 4.7717237 \\ \mathrm{H} & 3.5719606 & 1.8359716 & 7.1552496 \\ \mathrm{H} & 0.6404377 & -1.2336611 & 7.9511390 \\ \mathrm{H} & 0.7683843 & -1.9708347 & 5.5733286 \\ \mathrm{H} & 2.0469890 & 0.6666682 & 8.7459015 \\ \mathrm{C} & 2.3379066 & 0.8842534 & 0.6247069 \\ \mathrm{C} & 3.6947614 & 0.5646002 & 0.8441722 \\ \mathrm{C} & 4.3042755 & -1.8499516 & -1.7621532 \\ \mathrm{C} & 3.7175333 & -2.1235993 & -3.0059117 \\ \mathrm{C} & 2.3684977 & -1.8244052 & -3.2247111 \\ \mathrm{C} & 1.5902192 & -1.2533994 & -2.2063176 \\ \mathrm{C} & 1.9031693 & 2.2045243 & 0.7754186 \\ \mathrm{C} & 2.8226380 & 3.2001840 & 1.1384560 \\ \mathrm{C} & 4.1657163 & 2.8790295 & 1.3595893 \\ \mathrm{C} & 4.6052991 & 1.5552170 & 1.2159292 \\ \mathrm{H} & 5.3580464 & -2.0770010 & -1.5845765 \\ \mathrm{H} & 4.3157069 & -2.5686998 & -3.8031635 \\ \mathrm{H} & 1.9139127 & -2.0368096 & -4.1942668 \\ \mathrm{H} & 0.5350716 & -1.0436872 & -2.3822789 \\ \mathrm{H} & 0.8592458 & 2.4743642 & 0.6105347 \\ \mathrm{H} & 2.4835177 & 4.2318710 & 1.2475063 \\ \mathrm{H} & 4.8747893 & 3.6594406 & 1.6418322 \\ \mathrm{H} & 5.6536872 & 1.2998397 & 1.3855765 \\ \mathrm{O} & -0.7441547 & -1.1684009 & -0.2072140 \\ \mathrm{H} & -1.6814408 & -0.9103881 & -0.2630184 \\ \mathrm{H} & -0.0508219 & 0.6913685 & -0.8892935 \\ \mathrm{H} & -0.3779289 & 0.5870548 & 0.8646737\end{array}$

TS $4+B$ (endo)

Imaginary Frequencies $=1(-357.19 \mathrm{~cm}-1)$

Energy (BP86/def-TZVP, level of optimization) $=-974.7722555135 \mathrm{H}$ Energy (M0 6-2X/def2-TZVP) $=-974.3197608342 \mathrm{H}$

$\begin{array}{lrrr}\text { C } & 0.4710167 & 2.6359046 & 0.3695130 \\ \text { C } & 0.0096994 & 1.8980493 & 1.4603443 \\ \text { C } & -1.3416460 & 1.5851455 & 1.6266959 \\ \text { C } & -2.3248498 & 1.9925856 & 0.7144006 \\ \text { H } & 0.7489289 & 1.4297497 & 2.1142969 \\ \text { H } & -1.6181315 & 0.8760876 & 2.4115109 \\ \mathrm{C} & -0.2763273 & 1.3405020 & -1.4050284 \\ \mathrm{C} & -1.6058169 & 0.9979101 & -1.1305130 \\ \mathrm{C} & 0.5822258 & 0.1665137 & -1.1194443 \\ \mathrm{C} & -1.6271614 & -0.4157529 & -0.6669749 \\ \mathrm{O} & -2.5900623 & -1.1340696 & -0.4297133 \\ \mathrm{O} & 1.7762174 & 0.0092386 & -1.3452424 \\ \mathrm{~N} & -0.2703176 & -0.8189130 & -0.5540932 \\ \mathrm{C} & 0.1663170 & -2.1114631 & -0.1302075 \\ \mathrm{C} & -0.3676463 & -3.2633268 & -0.7243508 \\ \mathrm{C} & 0.0667778 & -4.5221182 & -0.2980656 \\ \mathrm{C} & 1.0390132 & -4.6331671 & 0.7027405 \\ \mathrm{C} & 1.5763351 & -3.4781256 & 1.2833780 \\ \mathrm{C} & 1.1375748 & -2.2157152 & 0.8745542 \\ \mathrm{H} & -1.1122949 & -3.1736653 & -1.5147069 \\ \mathrm{H} & -0.3519823 & -5.4188123 & -0.7578872\end{array}$




$\begin{array}{rrrr}\mathrm{H} & 2.3344508 & -3.5578601 & 2.0640695 \\ \mathrm{H} & 1.5430848 & -1.3138646 & 1.3338931 \\ \mathrm{H} & 1.3781094 & -5.6176811 & 1.0290586 \\ \mathrm{C} & 1.9215500 & 2.8324589 & 0.1690414 \\ \mathrm{H} & -0.1696843 & 3.3707049 & -0.1155611 \\ \mathrm{H} & -2.1437813 & 2.9111604 & 0.1516864 \\ \mathrm{C} & -3.7666733 & 1.6247706 & 0.8872189 \\ \mathrm{H} & -4.3174527 & 1.6714525 & -0.0612861 \\ \mathrm{H} & -3.8876841 & 0.6249780 & 1.3236935 \\ \mathrm{H} & -4.2386613 & 2.3541566 & 1.5681429 \\ \mathrm{O} & 2.8144088 & 2.2822846 & 0.8049212 \\ \mathrm{O} & 2.1703634 & 3.7185824 & -0.8342645 \\ \mathrm{H} & 3.1438946 & 3.8002956 & -0.9292820 \\ \mathrm{H} & 0.0502580 & 2.1001862 & -2.1098843 \\ \mathrm{H} & -2.4674406 & 1.4114106 & -1.6485048\end{array}$

TS $4+B$ (exo)

Imaginary Frequencies $=1(-367.99 \mathrm{~cm}-1)$

Energy(BP86/def-TZVP, level of optimization) $=-974.7679015017 \mathrm{H}$

Energy (M0 6-2X/def2-TZVP) $=-974.3155225294 \mathrm{H}$

$0.4553989-2.4541807$

C $\quad 0.2591720$

$0.4210546-3.4804088$

1.6187306

0.6400296

2.1426016

0.9131465

$-0.0569671$

0.0508727

2.3178444

0.1101242

0.4347423

$-1.2552811$

1.4665712

$-1.0040646$

$-3.2508738$

$-1.9836176$

$-4.4587006$

$-4.0663979$

$-1.1901816$

$-0.2719754$

$-1.9811363$

$-0.9416853$

$-0.6460508$

$-1.0054730$

$-1.9024501$

0.0688712

2.2943559

$-1.5180660$

$-1.4208290$

$\begin{array}{ll}-0.5562982 & 0.4706317\end{array}$

2. 6154861

$-0.3075077$

1.1050851

$-1.8223531$

$-1.2404111$

0.3116616

0.2811021

$-0.4706124$

0.9972049

$-0.0511984$

$-0.9980622$

$-0.0555909$

2.3232146

$0.9601655 \quad 2.5109193$

$-1.3400236$

1.3540536

3.8077805

$-0.7288107$

0.7485898

4.9122697

$-0.2606183$

4.7171558

0.5582825

$-0.6727233$

3.4241156

$-1.4603303$

1.4394312

1.6474069

$-2.0805892$

2.1419672

3.9537198

$-0.7384665$

5.5747426

0.6979268

$-1.4700731$

3.2683957

1.2845170
-0.9932422

1.0622656

5.9234197

$-2.0970625$

0.1156272

$-2.7566562$

$-0.5429954$

1.1172529

$-1.6001162$

1. 4978095

1.4229197

$-1.2624402$

3. 6125761

1.0518682

$-1.7433782$

3. 8855137

0.7145150

$-0.7334987$

4.2033700

0.4979529

$-2.4850890$

$\mathrm{H} \quad 3.8900275$

2.1181903

$-1.8053341$ 


$\begin{array}{llrr}\mathrm{O} & -2.4698663 & -0.6388154 & -3.6506431 \\ \mathrm{O} & -2.9568739 & 0.7442373 & -1.9151687 \\ \mathrm{H} & -3.8683632 & 0.4643479 & -2.1494313\end{array}$

\section{Product $4+B$ (endo)}

Imaginary Frequencies = 0

Energy (BP86/def-TZVP, level of optimization) $=-974.8327116455 \mathrm{H}$

Energy (M06-2X/def2-TZVP) $=-974.4018324357 \mathrm{H}$

C $\quad 1.4889103 \quad 0.2601669 \quad 0.2151841$

C $\quad 2.1108372-0.1221660 \quad-1.1012512$

$\begin{array}{llll}\text { C } & 3.4379161 & -0.3172516 & -1.1290117\end{array}$

C $\quad 4.2478980 \quad-0.1078681 \quad 0.1295930$

$\mathrm{H} \quad 1.4730505 \quad-0.2465921 \quad-1.9773401$

$\mathrm{H} \quad 3.9499528 \quad-0.6114150 \quad-2.0488125$

$\begin{array}{llll}\text { C } & 2.0168099 & -0.6936775 & 1.3283420\end{array}$

$\begin{array}{llll}\text { C } & 3.5580277 & -0.8355354 & 1.3407435\end{array}$

C $\quad 1.4872465 \quad-2.1104102 \quad 1.1228642$

$\begin{array}{llll}\text { C } & 3.8016841 & -2.3403850 & 1.3655737\end{array}$

$\begin{array}{llll}0 & 4.8641906 & -2.9226797 & 1.5166961\end{array}$

$\begin{array}{llll}0 & 0.3234115 & -2.4395715 & 0.9455804\end{array}$

N $\quad 2.5665642 \quad-2.9994846 \quad 1.1940676$

C $\quad 2.4279697 \quad-4.4283486 \quad 1.1391140$

$\begin{array}{llll}\text { C } & 2.7338250 & -5.1934255 & 2.2702729\end{array}$

C $\quad 2.6018497 \quad-6.5841942 \quad 2.2110868$

C $\quad 2.1597541 \quad-7.2003729 \quad 1.0342467$

$\begin{array}{llll}\text { C } & 1.8518557 & -6.4247892 & -0.0896619\end{array}$

C $\quad 1.9894262-5.0338576 \quad-0.0436325$

$\mathrm{H} \quad 3.0666007 \quad-4.7044171 \quad 3.1863658$

$\mathrm{H} \quad 2.8408069 \quad-7.1857749 \quad 3.0893856$

$\mathrm{H} \quad 1.5080162 \quad-6.9018063 \quad-1.0086971$

$\mathrm{H} \quad 1.7624041 \quad-4.4232955 \quad-0.9179624$

$\mathrm{H} \quad 2.0549923 \quad-8.2857260 \quad 0.9931607$

$\begin{array}{llll}\text { C } & -0.0301484 & 0.3628928 & 0.1769496\end{array}$

$\mathrm{H} \quad 1.8296201 \quad 1.2723417 \quad 0.5063759$

$\begin{array}{llll}\mathrm{H} & 4.1661403 & 0.9627697 & 0.3961192\end{array}$

C $\quad 5.7373917 \quad-0.4208666 \quad-0.0401612$

$\mathrm{H} \quad 6.2880000 \quad-0.2635095 \quad 0.8975959$

$\mathrm{H} \quad 5.9029923 \quad-1.4578911 \quad-0.3610790$

$\mathrm{H} \quad \begin{array}{llll}6.1655056 & 0.2457064 & -0.8029560\end{array}$

$\begin{array}{lllll}0 & -0.7237379 & 0.2299564 & -0.8190220\end{array}$

$\begin{array}{llll}0 & -0.5331879 & 0.7049908 & 1.3872972\end{array}$

$\begin{array}{llll}\mathrm{H} & -1.5076315 & 0.7875968 & 1.3003051\end{array}$

$\mathrm{H} \quad 1.6489060 \quad-0.3537721 \quad 2.3067627$

$\begin{array}{llll}\mathrm{H} & 3.9903724 & -0.4275938 & 2.2653138\end{array}$

\section{Product 4 + B (exo)}

Imaginary Frequencies $=0$

Energy (BP86/def-TZVP, level of optimization) $=-974.8349608909 \mathrm{H}$

Energy (M06-2X/def2-TZVP) $=-974.4020591138 \mathrm{H}$

$\begin{array}{lrrr}\mathrm{C} & 1.4119190 & 0.2462055 & 0.1596214 \\ \mathrm{C} & 2.2413195 & 0.0257244 & -1.0932449 \\ \mathrm{C} & 3.5717704 & -0.0866946 & -1.0108206 \\ \mathrm{C} & 4.3096824 & -0.0313230 & 0.3063597\end{array}$




$\begin{array}{rrrr}\text { H } & 1.7295500 & 0.0099548 & -2.0576106 \\ \mathrm{H} & 4.1666138 & -0.2048416 & -1.9206625 \\ \mathrm{C} & 1.9669935 & -0.5644184 & 1.3547231 \\ \mathrm{C} & 3.4920132 & -0.8143058 & 1.3805402 \\ \mathrm{H} & 1.4390130 & -1.5311050 & 1.3635698 \\ \mathrm{C} & 1.6433006 & 0.1110350 & 2.6787619 \\ \mathrm{H} & 3.7081454 & -1.8835391 & 1.2390193 \\ \mathrm{C} & 3.9124722 & -0.4510671 & 2.8005478 \\ \mathrm{O} & 5.0107954 & -0.5954409 & 3.3154208 \\ \mathrm{O} & 0.5705833 & 0.5889074 & 3.0189382 \\ \mathrm{~N} & 2.8018991 & 0.1037895 & 3.4692756 \\ \mathrm{C} & 2.8510079 & 0.5985754 & 4.8164934 \\ \mathrm{C} & 2.0953519 & -0.0339515 & 5.8099809 \\ \mathrm{C} & 2.1426006 & 0.4536415 & 7.1195513 \\ \mathrm{C} & 2.9446968 & 1.5577330 & 7.4317147 \\ \mathrm{C} & 3.7003328 & 2.1798284 & 6.4307874 \\ \mathrm{C} & 3.6534394 & 1.7048261 & 5.1165725 \\ \mathrm{H} & 1.4820365 & -0.9004664 & 5.5607156 \\ \mathrm{H} & 1.5540533 & -0.0347024 & 7.8976861 \\ \mathrm{H} & 4.3254946 & 3.0414407 & 6.6699824 \\ \mathrm{H} & 4.2301123 & 2.1903960 & 4.3287795 \\ \mathrm{H} & 2.9808869 & 1.9336518 & 8.4555075 \\ \mathrm{C} & -0.0464715 & -0.1045244 & -0.1136700 \\ \mathrm{H} & 1.4411563 & 1.3228965 & 0.3996191 \\ \mathrm{H} & 4.3528088 & 1.0284488 & 0.6229574 \\ \mathrm{C} & 5.7460714 & -0.5544556 & 0.1764381 \\ \mathrm{H} & 6.2850720 & -0.4846123 & 1.1291553 \\ \mathrm{H} & 5.7456789 & -1.6077954 & -0.1438410 \\ \mathrm{H} & 6.2936886 & 0.0311456 & -0.5760770 \\ \mathrm{O} & -0.5720499 & -1.1833972 & 0.1114499 \\ \mathrm{O} & -0.6947546 & 0.9284664 & -0.6979358 \\ \mathrm{H} & -1.6091283 & 0.6386280 & -0.9108900\end{array}$

\section{TS $5+B$ (endo)}

Imaginary Frequencies $=1(-387.48 \mathrm{~cm}-1)$

Energy (BP86/def-TZVP, level of optimization) $=-1315.7235789745 \mathrm{H}$

Energy (M06-2X/def2-TZVP) $=-1315.110804767 \mathrm{H}$

$\begin{array}{lrrr}\text { C } & -1.0406214 & 1.3504901 & 0.6648224 \\ \mathrm{C} & -0.6701817 & 0.7923070 & -0.5996368 \\ \mathrm{C} & 0.7031515 & 0.7452430 & -0.6212977 \\ \mathrm{C} & 1.1498046 & 1.2730363 & 0.6324388 \\ \mathrm{O} & 0.0841775 & 1.9605719 & 1.2017486 \\ \mathrm{H} & -1.3665668 & 0.3936767 & -1.3322667 \\ \mathrm{H} & 1.3468814 & 0.2958781 & -1.3724496 \\ \mathrm{C} & -2.3681792 & 1.9266518 & 1.0720669 \\ \mathrm{H} & -2.3568499 & 2.1639201 & 2.1441015 \\ \mathrm{H} & -3.1494179 & 1.1781221 & 0.8861854 \\ \mathrm{C} & 2.5247859 & 1.7669810 & 0.9900790 \\ \mathrm{H} & 2.5713324 & 1.9930091 & 2.0635220 \\ \mathrm{H} & 3.2507721 & 0.9742600 & 0.7664668 \\ \mathrm{~N} & -2.7206314 & 3.1256432 & 0.3179769 \\ \mathrm{~N} & 2.9172451 & 2.9535469 & 0.2360814 \\ \mathrm{C} & -3.6103110 & 3.1186949 & -0.7147938\end{array}$




$\begin{array}{lrrr}\mathrm{C} & 3.7248146 & 2.9031242 & -0.8606002 \\ \mathrm{C} & 4.0240499 & 4.2341748 & -1.5175105 \\ \mathrm{H} & 3.6541488 & 4.2087304 & -2.5522056 \\ \mathrm{H} & 3.5710896 & 5.0873328 & -0.9971946 \\ \mathrm{H} & 5.1131325 & 4.3735229 & -1.5576515 \\ \mathrm{O} & 4.1905499 & 1.8341697 & -1.2928389 \\ \mathrm{H} & 2.5538612 & 3.8540118 & 0.5368545 \\ \mathrm{C} & -3.8495864 & 4.4558768 & -1.3835136 \\ \mathrm{H} & -3.2816762 & 5.2771535 & -0.9286656 \\ \mathrm{H} & -3.5741574 & 4.3747688 & -2.4444017 \\ \mathrm{H} & -4.9220269 & 4.6900291 & -1.3358721 \\ \mathrm{O} & -4.1927775 & 2.0832925 & -1.0825659 \\ \mathrm{H} & -2.2670274 & 4.0002580 & 0.5684495 \\ \mathrm{C} & 0.7339242 & -0.3933245 & 1.8882199 \\ \mathrm{C} & -0.6819893 & -0.3592954 & 1.9122538 \\ \mathrm{H} & 1.3674321 & -0.0402353 & 2.7000189 \\ \mathrm{C} & 1.1502198 & -1.6070847 & 1.1419208 \\ \mathrm{H} & -1.2718908 & 0.0264320 & 2.7415391 \\ \mathrm{C} & -1.1806906 & -1.5483387 & 1.1808805 \\ \mathrm{O} & -2.3349779 & -1.9374024 & 1.0378311 \\ \mathrm{O} & 2.2814185 & -2.0379853 & 0.9466436 \\ \mathrm{~N} & -0.0386982 & -2.2155887 & 0.6606990 \\ \mathrm{C} & -0.0864266 & -3.3890654 & -0.1509212 \\ \mathrm{C} & 0.5788474 & -4.5511316 & 0.2643589 \\ \mathrm{C} & 0.5293294 & -5.6969328 & -0.5355515 \\ \mathrm{C} & -0.1923995 & -5.6909678 & -1.7349722 \\ \mathrm{C} & -0.8625321 & -4.5300297 & -2.1388864 \\ \mathrm{C} & -0.8065304 & -3.3750708 & -1.3533657 \\ \mathrm{H} & 1.1254543 & -4.5572639 & 1.2071399 \\ \mathrm{H} & 1.0502579 & -6.6000846 & -0.2134055 \\ \mathrm{H} & -1.4265299 & -4.5185280 & -3.0729855 \\ \mathrm{H} & -1.3163700 & -2.4659455 & -1.6721743 \\ \mathrm{H} & -0.2333407 & -6.5892868 & -2.3530673 \\ & & & \end{array}$

\section{TS $5+B$ (exo)}

Imaginary Frequencies $=1(-372.84 \mathrm{~cm}-1)$ Energy (BP86/def-TZVP, level of optimization) $=-1315.7219927474 \mathrm{H}$ Energy (M0 6-2X/def2-TZVP) $=-1315.112512856 \mathrm{H}$

$\begin{array}{lrrr}\text { C } & 1.1274727 & 1.1296366 & 0.5797242 \\ \mathrm{C} & 0.7690440 & 2.1879201 & 1.4761132 \\ \mathrm{C} & -0.6024687 & 2.2386424 & 1.4965652 \\ \mathrm{C} & -1.0645746 & 1.2076891 & 0.6146042 \\ \mathrm{O} & 0.0087765 & 0.8165328 & -0.1736463 \\ \mathrm{H} & 1.4712806 & 2.7376898 & 2.0970487 \\ \mathrm{H} & -1.2434659 & 2.8376193 & 2.1377151 \\ \mathrm{C} & 2.4571338 & 0.8990819 & -0.0826544 \\ \mathrm{H} & 2.4795432 & -0.0856329 & -0.5651156 \\ \mathrm{H} & 3.2422989 & 0.9278684 & 0.6837518 \\ \mathrm{C} & -2.4249639 & 1.0928636 & -0.0161301 \\ \mathrm{H} & -2.5326599 & 0.1260655 & -0.5228691 \\ \mathrm{H} & -3.1853183 & 1.1537099 & 0.7732391 \\ \mathrm{~N} & 2.7684066 & 1.9208006 & -1.0795488 \\ \mathrm{~N} & -2.6910798 & 2.1634088 & -0.9736777\end{array}$




$\begin{array}{lrrr}\mathrm{C} & 3.6006732 & 2.9711804 & -0.8356545 \\ \mathrm{C} & -3.4322108 & 3.2658727 & -0.6712088 \\ \mathrm{C} & -3.6005364 & 4.2706975 & -1.7914478 \\ \mathrm{H} & -3.2011060 & 5.2393522 & -1.4599989 \\ \mathrm{H} & -3.0972999 & 3.9745127 & -2.7203471 \\ \mathrm{H} & -4.6729416 & 4.4041403 & -1.9901949 \\ \mathrm{O} & -3.9451722 & 3.4355481 & 0.4490964 \\ \mathrm{H} & -2.2877294 & 2.0855761 & -1.9034159 \\ \mathrm{C} & 3.8175131 & 3.9192163 & -1.9966634 \\ \mathrm{H} & 3.2525273 & 3.6422535 & -2.8954525 \\ \mathrm{H} & 3.5248213 & 4.9318924 & -1.6862788 \\ \mathrm{H} & 4.8890370 & 3.9425362 & -2.2396029 \\ \mathrm{O} & 4.1554743 & 3.1390721 & 0.2648691 \\ \mathrm{H} & 2.3405635 & 1.8381119 & -1.9978484 \\ \mathrm{C} & 0.6883380 & -0.4603767 & 1.9994335 \\ \mathrm{C} & -0.7306826 & -0.4147482 & 1.9844846 \\ \mathrm{H} & 1.3118361 & -0.1280712 & 2.8252462 \\ \mathrm{C} & 1.1130163 & -1.6626238 & 1.2418320 \\ \mathrm{H} & -1.3475711 & -0.0692336 & 2.8102282 \\ \mathrm{C} & -1.2083256 & -1.5888351 & 1.2069279 \\ \mathrm{O} & -2.3598034 & -1.9756880 & 1.0310155 \\ \mathrm{O} & 2.2434179 & -2.1149924 & 1.0886482 \\ \mathrm{~N} & -0.0607734 & -2.2413359 & 0.6942944 \\ \mathrm{C} & -0.0887578 & -3.3674346 & -0.1831543 \\ \mathrm{C} & 0.5218316 & -4.5689150 & 0.2014264 \\ \mathrm{C} & 0.4943665 & -5.6642820 & -0.6674734 \\ \mathrm{C} & -0.1519639 & -5.5681789 & -1.9053844 \\ \mathrm{C} & -0.7680874 & -4.3677405 & -2.2790358 \\ \mathrm{C} & -0.7317334 & -3.2626153 & -1.4240110 \\ \mathrm{H} & 1.0096504 & -4.6439938 & 1.1730906 \\ \mathrm{H} & 0.9732632 & -6.5986305 & -0.3701214 \\ \mathrm{H} & -1.2724081 & -4.2863643 & -3.2433335 \\ \mathrm{H} & -1.1952102 & -2.3209018 & -1.7191310 \\ \mathrm{H} & -0.1754351 & -6.4272008 & -2.5778915 \\ & & & \\ & & & \\ & & & \end{array}$

\section{Product $5+B$ (endo)}

Imaginary Frequencies $=0$

Energy (BP86/def-TZVP, level of optimization) $=-1315.7552695972 \mathrm{H}$ Energy (M06-2X/def2-TZVP) $=-1315.158559793 \mathrm{H}$

$\begin{array}{lrrr}\mathrm{C} & -1.0381281 & 1.4490824 & 0.7539632 \\ \mathrm{C} & -0.6716124 & 1.1316504 & -0.6927071 \\ \mathrm{C} & 0.6676209 & 1.1103520 & -0.7395932 \\ \mathrm{C} & 1.1439378 & 1.4140464 & 0.6779766 \\ \mathrm{O} & 0.0820732 & 2.2861363 & 1.1644349 \\ \mathrm{H} & -1.3845000 & 0.8862285 & -1.4775849 \\ \mathrm{H} & 1.3154481 & 0.8412589 & -1.5716617 \\ \mathrm{C} & -2.3743302 & 2.1016643 & 1.0521613 \\ \mathrm{H} & -2.4299654 & 2.3385622 & 2.1255938 \\ \mathrm{H} & -3.1811527 & 1.3962734 & 0.8124112 \\ \mathrm{C} & 2.5169638 & 2.0248492 & 0.8823942 \\ \mathrm{H} & 2.6553403 & 2.2554623 & 1.9499317 \\ \mathrm{H} & 3.2839445 & 1.2972551 & 0.5849903 \\ \mathrm{~N} & -2.5968598 & 3.3049241 & 0.2670893\end{array}$




$\begin{array}{rrrr}\mathrm{N} & 2.7184503 & 3.2237801 & 0.0849855 \\ \mathrm{C} & -3.5439800 & 3.4026116 & -0.7072038 \\ \mathrm{C} & 3.6141669 & 3.3045732 & -0.9382658 \\ \mathrm{C} & 3.6835141 & 4.6419712 & -1.6456464 \\ \mathrm{H} & 3.4556004 & 4.4922954 & -2.7101752 \\ \mathrm{H} & 2.9929273 & 5.3883293 & -1.2330493 \\ \mathrm{H} & 4.7107291 & 5.0265713 & -1.5774572 \\ \mathrm{O} & 4.3350091 & 2.3467873 & -1.2724711 \\ \mathrm{H} & 2.1604509 & 4.0435343 & 0.3088800 \\ \mathrm{C} & -3.6261824 & 4.7415232 & -1.4103452 \\ \mathrm{H} & -2.9044085 & 5.4763663 & -1.0318564 \\ \mathrm{H} & -3.4526216 & 4.5890999 & -2.4847232 \\ \mathrm{H} & -4.6425328 & 5.1426097 & -1.2924447 \\ \mathrm{O} & -4.2986903 & 2.4581922 & -1.0022769 \\ \mathrm{H} & -2.0114610 & 4.1135727 & 0.4590821 \\ \mathrm{C} & 0.8310157 & 0.1319192 & 1.5631224 \\ \mathrm{C} & -0.7056496 & 0.1571416 & 1.6182238 \\ \mathrm{H} & 1.3118902 & 0.2327227 & 2.5447019 \\ \mathrm{C} & 1.1943309 & -1.2032095 & 0.9463140 \\ \mathrm{H} & -1.1123677 & 0.2753345 & 2.6308607 \\ \mathrm{C} & -1.1555015 & -1.1680428 & 1.0378311 \\ \mathrm{O} & -2.3008787 & -1.5683626 & 0.8950997 \\ \mathrm{O} & 2.3121160 & -1.6308906 & 0.7010991 \\ \mathrm{~N} & -0.0034334 & -1.8993906 & 0.6795896 \\ \mathrm{C} & -0.0448860 & -3.2049428 & 0.0824563 \\ \mathrm{C} & 0.4921862 & -4.2941483 & 0.7778842 \\ \mathrm{C} & 0.4547700 & -5.5640234 & 0.1942903 \\ \mathrm{C} & -0.1220421 & -5.7418118 & -1.0687657 \\ \mathrm{C} & -0.6606084 & -4.6460836 & -1.7535315 \\ \mathrm{C} & -0.6199307 & -3.3705175 & -1.1823622 \\ \mathrm{H} & 0.9283793 & -4.1487163 & 1.7667669 \\ \mathrm{H} & 0.8733375 & -6.4162530 & 0.7317813 \\ \mathrm{H} & -1.1098099 & -4.7807730 & -2.7386188 \\ \mathrm{H} & -1.0280847 & -2.5108610 & -1.7146755 \\ \mathrm{H} & -0.1526245 & -6.7351646 & -1.5195698\end{array}$

\section{Product $5+B$ (exo)}

Imaginary Frequencies $=0$

Energy (BP86/def-TZVP, level of optimization) $=-1315.7558942626 \mathrm{H}$

Energy (M0 6-2X/def2-TZVP) $=-1315.161813919 \mathrm{H}$

$\begin{array}{lrrr}\text { C } & 1.1255822 & 1.2689123 & 0.4980998 \\ \mathrm{C} & 0.7441692 & 2.5206461 & 1.2865755 \\ \mathrm{C} & -0.5927380 & 2.5381936 & 1.3298085 \\ \mathrm{C} & -1.0574014 & 1.2993010 & 0.5661230 \\ \mathrm{O} & 0.0022314 & 1.1375079 & -0.4197491 \\ \mathrm{H} & 1.4559438 & 3.1732633 & 1.7886696 \\ \mathrm{H} & -1.2533382 & 3.2090840 & 1.8758015 \\ \mathrm{C} & 2.4518827 & 1.2687081 & -0.2415153 \\ \mathrm{H} & 2.5482512 & 0.3544533 & -0.8435097 \\ \mathrm{H} & 3.2760307 & 1.2887645 & 0.4839339 \\ \mathrm{C} & -2.4272438 & 1.3379265 & -0.0883771 \\ \mathrm{H} & -2.5884245 & 0.4274259 & -0.6819913 \\ \mathrm{H} & -3.2022324 & 1.3812850 & 0.6884833\end{array}$




\begin{tabular}{|c|c|c|c|}
\hline $\mathrm{N}$ & 2.5924634 & 2.4373245 & -1.0980333 \\
\hline $\mathrm{N}$ & -2.5887021 & 2.5099111 & -0.9364005 \\
\hline $\mathrm{C}$ & 3.5171886 & 3.4156027 & -0.8961389 \\
\hline $\mathrm{C}$ & -3.4509137 & 3.5272888 & -0.6633101 \\
\hline C & -3.4696940 & 4.6580359 & -1.6707833 \\
\hline $\mathrm{H}$ & -3.2054696 & 5.5936839 & -1.1583230 \\
\hline $\mathrm{H}$ & -2.7807910 & 4.5032833 & -2.5106900 \\
\hline $\mathrm{H}$ & -4.4911697 & 4.7703689 & -2.0597024 \\
\hline 0 & -4.1828908 & 3.5337367 & 0.3435982 \\
\hline $\mathrm{H}$ & -2.0164656 & 2.5707768 & -1.7741071 \\
\hline C & 3.5046886 & 4.5459563 & -1.9041002 \\
\hline $\mathrm{H}$ & 2.7372288 & 4.4279056 & -2.6794168 \\
\hline $\mathrm{H}$ & 3.3351526 & 5.4929567 & -1.3729052 \\
\hline $\mathrm{H}$ & 4.4920883 & 4.6064246 & -2.3826132 \\
\hline 0 & 4.3266607 & 3.3884994 & 0.0492814 \\
\hline $\mathrm{H}$ & 1.9619351 & 2.5222931 & -1.8905871 \\
\hline C & 0.8209196 & 0.0648512 & 1.4871600 \\
\hline C & -0.7263929 & 0.0843430 & 1.5326982 \\
\hline $\mathrm{H}$ & 1.3191757 & 0.1971611 & 2.4548589 \\
\hline C & 1.1848547 & -1.2962912 & 0.9179150 \\
\hline $\mathrm{H}$ & -1.1635821 & 0.2258510 & 2.5282531 \\
\hline C & -1.1571985 & -1.2666742 & 0.9867112 \\
\hline 0 & -2.2964589 & -1.6967017 & 0.8872522 \\
\hline 0 & 2.3054465 & -1.7502878 & 0.7430788 \\
\hline $\mathrm{N}$ & -0.0049729 & -1.9835395 & 0.6154621 \\
\hline C & -0.0391987 & -3.2996913 & 0.0398829 \\
\hline C & 0.4508859 & -4.3872238 & 0.7710039 \\
\hline C & 0.4204435 & -5.6632870 & 0.2001882 \\
\hline $\mathrm{C}$ & -0.1037265 & -5.8478116 & -1.0845930 \\
\hline C & -0.5964603 & -4.7530823 & -1.8046167 \\
\hline C & -0.5625915 & -3.4718740 & -1.2461287 \\
\hline $\mathrm{H}$ & 0.8470425 & -4.2358328 & 1.7756402 \\
\hline $\mathrm{H}$ & 0.8038379 & -6.5148404 & 0.7642894 \\
\hline $\mathrm{H}$ & -1.0042166 & -4.8932431 & -2.8068339 \\
\hline $\mathrm{H}$ & -0.9333208 & -2.6117369 & -1.8048145 \\
\hline $\mathrm{H}$ & -0.1276990 & -6.8456881 & -1.5256284 \\
\hline
\end{tabular}

TS $1+C$

Imaginary Frequencies $=1(-366.37 \mathrm{~cm}-1)$

Energy (BP86/def-TZVP, level of optimization) $=-1358.4153538525 \mathrm{H}$

Energy (M0 6-2X/def2-TZVP) $=-1357.827977265 \mathrm{H}$

$\begin{array}{lrrr}\text { C } & -1.1104293 & -0.0041750 & -1.6866015 \\ \text { C } & -0.2320145 & 0.0749594 & -2.8458631 \\ \text { C } & 1.0359158 & 0.6977124 & -2.6465664 \\ \mathrm{C} & 1.3413412 & 1.1460478 & -1.3143853 \\ \mathrm{C} & -2.3620291 & -0.8307521 & -1.7983884 \\ \mathrm{H} & 2.3360188 & 1.5614099 & -1.1331070 \\ \mathrm{C} & 0.3528478 & -1.4582674 & -0.5213488 \\ \mathrm{C} & 1.6121119 & -0.8218014 & -0.4367471 \\ \mathrm{H} & 0.0946341 & -2.2386998 & -1.2307206 \\ \mathrm{C} & -0.2827210 & -1.4543511 & 0.8094300 \\ \mathrm{C} & 1.7759083 & -0.3883469 & 0.9953414 \\ \mathrm{O} & 2.7488630 & 0.1498211 & 1.5070404\end{array}$




$\begin{array}{rrrr}\mathrm{O} & -1.3333891 & -1.9769507 & 1.1661228 \\ \mathrm{~N} & 0.5911476 & -0.7388926 & 1.6779230 \\ \mathrm{C} & 0.3624128 & -0.5432442 & 3.0744938 \\ \mathrm{C} & -0.8360959 & 0.0356829 & 3.5118734 \\ \mathrm{C} & -1.0634784 & 0.2006500 & 4.8812474 \\ \mathrm{C} & -0.0965258 & -0.1985481 & 5.8112359 \\ \mathrm{C} & 1.1003271 & -0.7734519 & 5.3671507 \\ \mathrm{C} & 1.3315049 & -0.9561299 & 4.0008117 \\ \mathrm{H} & -1.5779107 & 0.3603656 & 2.7833698 \\ \mathrm{H} & -1.9984409 & 0.6492519 & 5.2210509 \\ \mathrm{H} & 1.8560144 & -1.0924788 & 6.0868607 \\ \mathrm{H} & 2.2543957 & -1.4202370 & 3.6546557 \\ \mathrm{H} & -0.2752592 & -0.0631815 & 6.8792519 \\ \mathrm{C} & -1.0373075 & 1.0792580 & -0.7168849 \\ \mathrm{C} & 0.2540066 & 1.6654611 & -0.5293334 \\ \mathrm{C} & 1.9564970 & 0.7891297 & -3.7135049 \\ \mathrm{C} & 1.6148387 & 0.3145022 & -4.9722289 \\ \mathrm{C} & 0.3578797 & -0.2889034 & -5.1786474 \\ \mathrm{C} & -0.5461329 & -0.4232143 & -4.1328674 \\ \mathrm{C} & -2.1094611 & 1.5488546 & 0.0839414 \\ \mathrm{C} & -1.9098686 & 2.5838861 & 0.9881542 \\ \mathrm{C} & -0.6336431 & 3.1549295 & 1.1698743 \\ \mathrm{C} & 0.4417562 & 2.6830776 & 0.4335116 \\ \mathrm{H} & 2.9258340 & 1.2608649 & -3.5411339 \\ \mathrm{H} & 2.3162092 & 0.4106846 & -5.8025302 \\ \mathrm{H} & 0.0880302 & -0.6527226 & -6.1713846 \\ \mathrm{H} & -1.5096008 & -0.8962630 & -4.3170167 \\ \mathrm{H} & -3.0999669 & 1.1196286 & -0.0420120 \\ \mathrm{H} & -2.7565108 & 2.9582859 & 1.5663463 \\ \mathrm{H} & -0.4940772 & 3.9650310 & 1.8872077 \\ \mathrm{H} & 1.4380076 & 3.1092896 & 0.5653853 \\ \mathrm{O} & -2.1130980 & -2.0803743 & -2.2580455 \\ \mathrm{H} & -2.9741663 & -2.5472861 & -2.3339049 \\ \mathrm{C} & 2.8416474 & -1.2829505 & -1.1768913 \\ \mathrm{H} & 3.6601616 & -0.5558239 & -1.0945509 \\ \mathrm{H} & 3.1924970 & -2.2351360 & -0.7493374 \\ \mathrm{H} & 2.6226822 & -1.4520639 & -2.2384069 \\ \mathrm{O} & -3.4938198 & -0.4520961 & -1.5242486 \\ & & & \\ & & & \end{array}$

\section{Product $1+C$}

Imaginary Frequencies $=0$

Energy (BP86/def-TZVP, level of optimization) $=-1358.4682658667 \mathrm{H}$ Energy (M06-2X/def2-TZVP) $=-1357.898967570 \mathrm{H}$

$\begin{array}{lllr}\text { C } & 1.4815128 & -0.3511962 & 0.2478946 \\ \text { C } & 2.1687792 & -0.9616172 & -0.9910296 \\ \text { C } & 3.5286383 & -1.2551341 & -0.7812821 \\ \text { C } & 4.0160509 & -0.9248997 & 0.6121394 \\ \text { C } & 0.0104158 & -0.0769943 & -0.0666756 \\ \text { H } & 5.0828112 & -1.1395974 & 0.7511044 \\ \text { C } & 1.6612209 & -1.4050607 & 1.4143285 \\ \text { C } & 3.1485494 & -1.7964909 & 1.6012408 \\ \text { H } & 1.0378402 & -2.2828443 & 1.2010505 \\ \text { C } & 1.2193235 & -0.8184254 & 2.7517071\end{array}$




$\begin{array}{rrrr}\mathrm{C} & 3.4661452 & -1.4065700 & 3.0476171 \\ \mathrm{O} & 4.5359573 & -1.5357725 & 3.6208207 \\ \mathrm{O} & 0.1271529 & -0.3471914 & 3.0260207 \\ \mathrm{~N} & 2.3024885 & -0.8879921 & 3.6442573 \\ \mathrm{C} & 2.2381721 & -0.4332950 & 5.0057684 \\ \mathrm{C} & 3.0371942 & 0.6402582 & 5.4134256 \\ \mathrm{C} & 2.9724550 & 1.0749502 & 6.7405702 \\ \mathrm{C} & 2.1091731 & 0.4469527 & 7.6459520 \\ \mathrm{C} & 1.3109063 & -0.6232560 & 7.2256535 \\ \mathrm{C} & 1.3752552 & -1.0720009 & 5.9031179 \\ \mathrm{H} & 3.6991164 & 1.1310547 & 4.6992577 \\ \mathrm{H} & 3.5951898 & 1.9102556 & 7.0644767 \\ \mathrm{H} & 0.6380477 & -1.1160602 & 7.9290721 \\ \mathrm{H} & 0.7650455 & -1.9125045 & 5.5706309 \\ \mathrm{H} & 2.0584387 & 0.7918612 & 8.6799335 \\ \mathrm{C} & 2.3235276 & 0.8666164 & 0.6635230 \\ \mathrm{C} & 3.6799552 & 0.5315532 & 0.8687924 \\ \mathrm{C} & 4.2999489 & -1.7951976 & -1.8112802 \\ \mathrm{C} & 3.7122210 & -2.0358286 & -3.0609546 \\ \mathrm{C} & 2.3607880 & -1.7431421 & -3.2693704 \\ \mathrm{C} & 1.5817449 & -1.2065789 & -2.2336569 \\ \mathrm{C} & 1.8917250 & 2.1756877 & 0.8986233 \\ \mathrm{C} & 2.8163470 & 3.1448477 & 1.3146256 \\ \mathrm{C} & 4.1610780 & 2.8133111 & 1.5062215 \\ \mathrm{C} & 4.5949440 & 1.4990495 & 1.2885580 \\ \mathrm{H} & 5.3547201 & -2.0226176 & -1.6422244 \\ \mathrm{H} & 4.3123079 & -2.4515964 & -3.8721190 \\ \mathrm{H} & 1.9049646 & -1.9291239 & -4.2432639 \\ \mathrm{H} & 0.5287567 & -0.9840369 & -2.4157203 \\ \mathrm{H} & 0.8489363 & 2.4477108 & 0.7492882 \\ \mathrm{H} & 2.4771899 & 4.1676029 & 1.4881979 \\ \mathrm{H} & 4.8734581 & 3.5755167 & 1.8269375 \\ \mathrm{H} & 5.6416155 & 1.2285572 & 1.4431983 \\ \mathrm{O} & -0.7522030 & -1.1832078 & 0.0786699 \\ \mathrm{H} & -1.6693371 & -0.9567033 & -0.1912467 \\ \mathrm{C} & 3.4180710 & -3.2993127 & 1.4246667 \\ \mathrm{H} & 4.4762615 & -3.5232907 & 1.6166020 \\ \mathrm{H} & 2.8053674 & -3.8912463 & 2.1195901 \\ \mathrm{H} & 3.1716835 & -3.6084883 & 0.4004448 \\ \mathrm{O} & -0.4323523 & 0.9797482 & -0.4868849 \\ & & & \end{array}$

TS $2+C$

Imaginary Frequencies $=1(-373.06 \mathrm{~cm}-1)$

Energy (BP86/def-TZVP, level of optimization) $=-1169.7550524933 \mathrm{H}$

Energy (M06-2X/def2-TZVP) $=-1169.244336998 \mathrm{H}$

$\begin{array}{lrrr}\text { C } & -1.0003650 & -1.1429545 & -1.6914450 \\ \mathrm{C} & -0.4983275 & -0.6462833 & -2.9499998 \\ \mathrm{C} & -0.0124233 & 0.6964047 & -2.9590571 \\ \mathrm{C} & -0.0804669 & 1.4232471 & -1.7252705 \\ \mathrm{H} & -1.3256841 & -2.1854453 & -1.6509447 \\ \mathrm{H} & 0.3880669 & 2.4093754 & -1.6769131 \\ \mathrm{C} & 0.9310267 & -1.2685340 & -0.7167686 \\ \mathrm{C} & 1.5845774 & -0.0135661 & -0.7511799\end{array}$




$\begin{array}{lrrr}\text { H } & 1.2266407 & -2.1152344 & -1.3339322 \\ \mathrm{C} & 0.5371547 & -1.5457722 & 0.6896972 \\ \mathrm{C} & 1.5178825 & 0.5607011 & 0.6176736 \\ \mathrm{O} & 1.9835134 & 1.6202972 & 1.0242612 \\ \mathrm{O} & 0.0752391 & -2.5784873 & 1.1627267 \\ \mathrm{~N} & 0.8310136 & -0.3789345 & 1.4362241 \\ \mathrm{C} & 0.6286202 & -0.2416282 & 2.8430066 \\ \mathrm{C} & 1.7016290 & 0.1298197 & 3.6673031 \\ \mathrm{C} & 1.5020469 & 0.2566886 & 5.0449678 \\ \mathrm{C} & 0.2453355 & -0.0019311 & 5.6049488 \\ \mathrm{C} & -0.8171863 & -0.3850302 & 4.7782984 \\ \mathrm{C} & -0.6320205 & -0.4996949 & 3.3973700 \\ \mathrm{H} & 2.6838350 & 0.3139832 & 3.2337348 \\ \mathrm{H} & 2.3372868 & 0.5494235 & 5.6834662 \\ \mathrm{H} & -1.7994388 & -0.5895336 & 5.2077423 \\ \mathrm{H} & -1.4605060 & -0.7796112 & 2.7484126 \\ \mathrm{H} & 0.0953309 & 0.0930199 & 6.6817433 \\ \mathrm{C} & -1.6463096 & -0.2071382 & -0.8063905 \\ \mathrm{C} & -1.1340903 & 1.1263047 & -0.8019402 \\ \mathrm{C} & 0.5701707 & 1.2149747 & -4.1407708 \\ \mathrm{C} & 0.6320513 & 0.4335720 & -5.2873399 \\ \mathrm{C} & 0.1546061 & -0.8954419 & -5.2739590 \\ \mathrm{C} & -0.3862732 & -1.4372159 & -4.1135082 \\ \mathrm{C} & -2.6939798 & -0.5561333 & 0.0760054 \\ \mathrm{C} & -3.2429928 & 0.4042623 & 0.9148037 \\ \mathrm{C} & -2.7227460 & 1.7184880 & 0.9363927 \\ \mathrm{C} & -1.6690833 & 2.0722970 & 0.1069839 \\ \mathrm{H} & 0.9504241 & 2.2383678 & -4.1459168 \\ \mathrm{H} & 1.0578187 & 0.8456153 & -6.2038865 \\ \mathrm{H} & 0.2135848 & -1.5003067 & -6.1803908 \\ \mathrm{H} & -0.7442336 & -2.4687054 & -4.0978951 \\ \mathrm{H} & -3.0840712 & -1.5759097 & 0.0693806 \\ \mathrm{H} & -4.0776571 & 0.1435619 & 1.5678907 \\ \mathrm{H} & -3.1575157 & 2.4595929 & 1.6092595 \\ \mathrm{H} & -1.2689836 & 3.0881100 & 0.1192227 \\ \mathrm{C} & 2.7354784 & 0.3490199 & -1.6427472 \\ \mathrm{H} & 2.9508379 & 1.4248850 & -1.6036060 \\ \mathrm{H} & 3.6430868 & -0.1877838 & -1.3194457 \\ \mathrm{H} & 2.5386691 & 0.0667277 & -2.6849138 \\ & & & \\ & & & \\ & -1.0630 & \end{array}$

\section{Product $2+\mathrm{C}$}

Imaginary Frequencies $=0$

Energy (BP86/def-TZVP, level of optimization) $=-1169.787288372 \mathrm{H}$

Energy (M06-2X/def2-TZVP) $=-1169.316719114 \mathrm{H}$

$\begin{array}{lrrr}\mathrm{C} & -0.6650809 & -1.2080575 & -1.4893678 \\ \mathrm{C} & -0.3366831 & -0.7233998 & -2.8908985 \\ \mathrm{C} & 0.1815643 & 0.5869091 & -2.9171937 \\ \mathrm{C} & 0.3042399 & 1.2095756 & -1.5371599 \\ \mathrm{H} & -1.0818977 & -2.2226810 & -1.4701792 \\ \mathrm{H} & 0.6966050 & 2.2341828 & -1.5596059 \\ \mathrm{C} & 0.6906660 & -1.1573650 & -0.6931500 \\ \mathrm{C} & 1.2826147 & 0.2727209 & -0.7186369 \\ \mathrm{H} & 1.3780801 & -1.8986858 & -1.1265938\end{array}$




$\begin{array}{lrrr}\mathrm{C} & 0.4922079 & -1.5025758 & 0.7779839 \\ \mathrm{C} & 1.3023903 & 0.7053696 & 0.7536274 \\ \mathrm{O} & 1.6589794 & 1.7878131 & 1.1798064 \\ \mathrm{O} & 0.0828752 & -2.5572117 & 1.2259033 \\ \mathrm{~N} & 0.8632651 & -0.3772534 & 1.5500167 \\ \mathrm{C} & 0.7801862 & -0.3294183 & 2.9796347 \\ \mathrm{C} & 1.8683967 & 0.1435865 & 3.7229672 \\ \mathrm{C} & 1.7798963 & 0.1933424 & 5.1155362 \\ \mathrm{C} & 0.6179311 & -0.2336032 & 5.7653690 \\ \mathrm{C} & -0.4627995 & -0.7071517 & 5.0153415 \\ \mathrm{C} & -0.3894884 & -0.7531250 & 3.6217670 \\ \mathrm{H} & 2.7705284 & 0.4788192 & 3.2134082 \\ \mathrm{H} & 2.6263731 & 0.5666386 & 5.6939833 \\ \mathrm{H} & -1.3732476 & -1.0409002 & 5.5155556 \\ \mathrm{H} & -1.2290079 & -1.1190052 & 3.0330481 \\ \mathrm{H} & 0.5544609 & -0.1961589 & 6.8540153 \\ \mathrm{C} & -1.5891466 & -0.1818345 & -0.8583749 \\ \mathrm{C} & -1.0629730 & 1.1241105 & -0.8812198 \\ \mathrm{C} & 0.5317921 & 1.1777945 & -4.1308598 \\ \mathrm{C} & 0.3591406 & 0.4596679 & -5.3230467 \\ \mathrm{C} & -0.1512725 & -0.8415894 & -5.2955535 \\ \mathrm{C} & -0.4986665 & -1.4399980 & -4.0758274 \\ \mathrm{C} & -2.8373675 & -0.4285725 & -0.2875888 \\ \mathrm{C} & -3.5667907 & 0.6380803 & 0.2552540 \\ \mathrm{C} & -3.0432302 & 1.9345483 & 0.2357658 \\ \mathrm{C} & -1.7849488 & 2.1820545 & -0.3289964 \\ \mathrm{H} & 0.9327256 & 2.1936115 & -4.1521867 \\ \mathrm{H} & 0.6240344 & 0.9200698 & -6.2763757 \\ \mathrm{H} & -0.2840091 & -1.3937784 & -6.2274259 \\ \mathrm{H} & -0.9002270 & -2.4555925 & -4.0544046 \\ \mathrm{H} & -3.2416988 & -1.4429347 & -0.2670240 \\ \mathrm{H} & -4.5480825 & 0.4545058 & 0.6963094 \\ \mathrm{H} & -3.6161597 & 2.7587023 & 0.6639639 \\ \mathrm{H} & -1.3704903 & 3.1922130 & -0.3353548 \\ \mathrm{C} & 2.7151853 & 0.3416069 & -1.2694073 \\ \mathrm{H} & 3.1128941 & 1.3614159 & -1.1700599 \\ \mathrm{H} & 3.3820464 & -0.3394865 & -0.7200763 \\ \mathrm{H} & 2.7283260 & 0.0554883 & -2.3298635 \\ & & & \end{array}$

TS $3+C$

Imaginary Frequencies $=1(-337.72 \mathrm{~cm}-1)$

Energy (BP86/def-TZVP, level of optimization) $=-1284.3361709707 \mathrm{H}$

Energy $($ M0 6-2X/def2-TZVP) $=-1283.779832590 \mathrm{H}$

$\begin{array}{lrrr}\text { C } & -1.2643110 & -0.3344201 & -1.7747761 \\ \text { C } & -0.4133854 & -0.1178840 & -2.9308380 \\ \text { C } & 0.7159484 & 0.7415476 & -2.7752865 \\ \text { C } & 0.9582332 & 1.2737843 & -1.4602314 \\ \text { C } & -2.3574725 & -1.3860255 & -1.9091056 \\ \text { H } & 1.8505433 & 1.8883439 & -1.3155997 \\ \mathrm{C} & 0.5130255 & -1.4587255 & -0.5999015 \\ \text { C } & 1.6432139 & -0.6061202 & -0.5959312 \\ \text { H } & 0.3670748 & -2.2950957 & -1.2784926 \\ \text { C } & -0.0335660 & -1.5240156 & 0.7570041\end{array}$




\begin{tabular}{|c|c|c|c|}
\hline C & 1.8066652 & -0.1183993 & 0.8164844 \\
\hline O & 2.6863922 & 0.6003643 & 1.2708868 \\
\hline 0 & -0.9798406 & -2.1986326 & 1.1839544 \\
\hline $\mathrm{N}$ & 0.7410506 & -0.6652927 & 1.5704361 \\
\hline C & 0.5301228 & -0.4505818 & 2.9675214 \\
\hline C & 1.5826291 & -0.6714590 & 3.8672544 \\
\hline $\mathrm{C}$ & 1.3748236 & -0.4641825 & 5.2339992 \\
\hline $\mathrm{C}$ & 0.1212676 & -0.0549104 & 5.7040756 \\
\hline C & -0.9269111 & 0.1543524 & 4.8001479 \\
\hline C & -0.7253318 & -0.0352019 & 3.4299421 \\
\hline $\mathrm{H}$ & 2.5521770 & -1.0069190 & 3.5001206 \\
\hline $\mathrm{H}$ & 2.1950147 & -0.6330410 & 5.9336602 \\
\hline $\mathrm{H}$ & -1.9059748 & 0.4739956 & 5.1607277 \\
\hline $\mathrm{H}$ & -1.5322766 & 0.1437578 & 2.7200010 \\
\hline $\mathrm{H}$ & -0.0384181 & 0.0999034 & 6.7724671 \\
\hline C & -1.3504526 & 0.7478906 & -0.8175627 \\
\hline C & -0.1943122 & 1.5753722 & -0.6543766 \\
\hline $\mathrm{C}$ & 1.5870911 & 0.9672293 & -3.8634154 \\
\hline $\mathrm{C}$ & 1.3332474 & 0.3830257 & -5.0973818 \\
\hline $\mathrm{C}$ & 0.2208896 & -0.4676455 & -5.2580745 \\
\hline $\mathrm{C}$ & -0.6296296 & -0.7292000 & -4.1912253 \\
\hline C & -2.5050520 & 1.0357450 & -0.0429021 \\
\hline C & -2.5123120 & 2.1120963 & 0.8328567 \\
\hline $\mathrm{C}$ & -1.3598576 & 2.9077098 & 1.0081616 \\
\hline C & -0.2088878 & 2.6305228 & 0.2857861 \\
\hline $\mathrm{H}$ & 2.4502952 & 1.6218411 & -3.7268804 \\
\hline $\mathrm{H}$ & 1.9942045 & 0.5812881 & -5.9427949 \\
\hline $\mathrm{H}$ & 0.0218264 & -0.9224986 & -6.2298557 \\
\hline $\mathrm{H}$ & -1.4851973 & -1.3857728 & -4.3480265 \\
\hline $\mathrm{H}$ & -3.3913881 & 0.4139063 & -0.1538565 \\
\hline $\mathrm{H}$ & -3.4193514 & 2.3437497 & 1.3940535 \\
\hline $\mathrm{H}$ & -1.3779084 & 3.7458433 & 1.7068880 \\
\hline $\mathrm{H}$ & 0.6844398 & 3.2469440 & 0.4060952 \\
\hline 0 & -2.9786273 & -1.8223888 & -0.7010917 \\
\hline $\mathrm{H}$ & -2.2721570 & -2.1172660 & -0.0740240 \\
\hline $\mathrm{H}$ & -1.9407588 & -2.2495619 & -2.4547020 \\
\hline $\mathrm{H}$ & -3.1648129 & -0.9737153 & -2.5391807 \\
\hline C & 2.8935644 & -0.8557400 & -1.3967293 \\
\hline $\mathrm{H}$ & 3.5625529 & 0.0147183 & -1.3840786 \\
\hline $\mathrm{H}$ & 3.4420166 & -1.7088085 & -0.9662300 \\
\hline $\mathrm{H}$ & 2.6538698 & -1.1025362 & -2.4380558 \\
\hline
\end{tabular}

\section{Product $3+\mathrm{C}$}

Imaginary Frequencies $=0$

Energy (BP86/def-TZVP, level of optimization) $=-1284.3867080331 \mathrm{H}$

Energy (M0 6-2X/def2-TZVP) $=-1283.846902987 \mathrm{H}$

$\begin{array}{lrrr}\text { C } & 1.4825138 & -0.3464421 & 0.2501840 \\ \text { C } & 2.1714413 & -0.9669115 & -0.9751743 \\ \text { C } & 3.5319734 & -1.2683583 & -0.7728567 \\ \text { C } & 4.0235547 & -0.9349508 & 0.6197228 \\ \text { C } & 0.0180251 & 0.0183796 & 0.0180113 \\ \text { H } & 5.0883171 & -1.1588198 & 0.7617381 \\ \text { C } & 1.6565007 & -1.4105982 & 1.4167662\end{array}$




$\begin{array}{rrrr}\mathrm{C} & 3.1475287 & -1.7932949 & 1.6124245 \\ \mathrm{H} & 1.0348705 & -2.2814436 & 1.1664798 \\ \mathrm{C} & 1.1956344 & -0.8836107 & 2.7695431 \\ \mathrm{C} & 3.4667312 & -1.3827622 & 3.0513514 \\ \mathrm{O} & 4.5442824 & -1.4825562 & 3.6187923 \\ \mathrm{O} & 0.0759730 & -0.5118130 & 3.0865025 \\ \mathrm{~N} & 2.2963819 & -0.8902139 & 3.6501204 \\ \mathrm{C} & 2.2250885 & -0.4453953 & 5.0137834 \\ \mathrm{C} & 2.9932551 & 0.6505648 & 5.4218671 \\ \mathrm{C} & 2.9259797 & 1.0766229 & 6.7517259 \\ \mathrm{C} & 2.0896623 & 0.4179219 & 7.6606448 \\ \mathrm{C} & 1.3218618 & -0.6745213 & 7.2406878 \\ \mathrm{C} & 1.3900025 & -1.1148525 & 5.9154351 \\ \mathrm{H} & 3.6340576 & 1.1650999 & 4.7050656 \\ \mathrm{H} & 3.5254541 & 1.9289883 & 7.0751865 \\ \mathrm{H} & 0.6700423 & -1.1914458 & 7.9466780 \\ \mathrm{H} & 0.8037086 & -1.9724344 & 5.5840585 \\ \mathrm{H} & 2.0362677 & 0.7560074 & 8.6967719 \\ \mathrm{C} & 2.3440798 & 0.8549656 & 0.6531816 \\ \mathrm{C} & 3.7001617 & 0.5274107 & 0.8621334 \\ \mathrm{C} & 4.3039330 & -1.8094205 & -1.8021232 \\ \mathrm{C} & 3.7159941 & -2.0446731 & -3.0529994 \\ \mathrm{C} & 2.3652545 & -1.7446116 & -3.2590334 \\ \mathrm{C} & 1.5873860 & -1.2087557 & -2.2213963 \\ \mathrm{C} & 1.9126402 & 2.1730745 & 0.8277057 \\ \mathrm{C} & 2.8362241 & 3.1602306 & 1.2040872 \\ \mathrm{C} & 4.1793369 & 2.8317656 & 1.4138320 \\ \mathrm{C} & 4.6150391 & 1.5093182 & 1.2458797 \\ \mathrm{H} & 5.3586868 & -2.0390166 & -1.6338766 \\ \mathrm{H} & 4.3141784 & -2.4614908 & -3.8653913 \\ \mathrm{H} & 1.9090651 & -1.9279985 & -4.2337583 \\ \mathrm{H} & 0.5314721 & -0.9958885 & -2.3887541 \\ \mathrm{H} & 0.8687168 & 2.4480464 & 0.6712496 \\ \mathrm{H} & 2.5002732 & 4.1907339 & 1.3323306 \\ \mathrm{H} & 4.8916984 & 3.6054433 & 1.7061898 \\ \mathrm{H} & 5.6633948 & 1.2485613 & 1.4070144 \\ \mathrm{O} & -0.7398057 & -1.1800986 & -0.2149356 \\ \mathrm{H} & -1.6777299 & -0.9221514 & -0.2586982 \\ \mathrm{H} & -0.0477918 & 0.6956860 & -0.8526668 \\ \mathrm{H} & -0.3739128 & 0.5496625 & 0.8980917 \\ \mathrm{C} & 3.4226205 & -3.2977101 & 1.4570943 \\ \mathrm{H} & 4.4791115 & -3.5181462 & 1.6634227 \\ \mathrm{H} & 2.8027723 & -3.8838721 & 2.1508533 \\ & 3.1886870 & -3.6178473 & 0.4331207\end{array}$

\section{TS $4+C$ (endo)}

Imaginary Frequencies $=1(-360.32 \mathrm{~cm}-1)$

Energy (BP86/def-TZVP, level of optimization) $=-1014.1024511739 \mathrm{H}$

Energy (M0 6-2X/def2-TZVP) $=-1013.632800577 \mathrm{H}$

$\begin{array}{rrrr}\text { C } & 0.8032858 & 2.2349304 & 0.7884467 \\ \text { C } & 0.1302519 & 1.4892577 & 1.7492881 \\ \text { C } & -1.2629742 & 1.4514393 & 1.8405741 \\ \text { C } & -2.1027915 & 2.1172350 & 0.9231496\end{array}$




$\begin{array}{rrrr}\mathrm{H} & 0.7164972 & 0.8122036 & 2.3753362 \\ \mathrm{H} & -1.7172132 & 0.7481338 & 2.5435973 \\ \mathrm{C} & -0.3681271 & 1.4047861 & -1.3283710 \\ \mathrm{C} & -1.6695276 & 1.1502457 & -0.8565854 \\ \mathrm{C} & 0.4260167 & 0.1659249 & -1.1867652 \\ \mathrm{C} & -1.7387666 & -0.2937352 & -0.4638600 \\ \mathrm{O} & -2.7215131 & -0.9447310 & -0.1322262 \\ \mathrm{O} & 1.5586947 & -0.0763106 & -1.5899984 \\ \mathrm{~N} & -0.4222808 & -0.7921736 & -0.5498147 \\ \mathrm{C} & -0.0124391 & -2.1150848 & -0.2039209 \\ \mathrm{C} & -0.7384748 & -3.2236194 & -0.6621025 \\ \mathrm{C} & -0.3252388 & -4.5113319 & -0.3062151 \\ \mathrm{C} & 0.8137513 & -4.6959554 & 0.4859976 \\ \mathrm{C} & 1.5415243 & -3.5844318 & 0.9280547 \\ \mathrm{C} & 1.1291175 & -2.2924106 & 0.5912581 \\ \mathrm{H} & -1.6155299 & -3.0793007 & -1.2916564 \\ \mathrm{H} & -0.8937156 & -5.3736006 & -0.6587400 \\ \mathrm{H} & 2.4312287 & -3.7211525 & 1.5449496 \\ \mathrm{H} & 1.6865096 & -1.4229181 & 0.9417959 \\ \mathrm{H} & 1.1344300 & -5.7031264 & 0.7571647 \\ \mathrm{C} & 2.2566079 & 2.0923768 & 0.6287369 \\ \mathrm{H} & 0.3511909 & 3.1130659 & 0.3323452 \\ \mathrm{H} & -1.7263355 & 3.0473939 & 0.4884859 \\ \mathrm{C} & -3.5977221 & 2.0641246 & 1.0864634 \\ \mathrm{H} & -4.1213885 & 2.3891220 & 0.1778754 \\ \mathrm{H} & -3.9414395 & 1.0567639 & 1.3563230 \\ \mathrm{H} & -3.8906643 & 2.7521909 & 1.8966310 \\ \mathrm{O} & 2.9542182 & 1.2221411 & 1.1466366 \\ \mathrm{O} & 2.7740688 & 3.0526436 & -0.1890191 \\ \mathrm{H} & 3.7382253 & 2.8877841 & -0.2699516 \\ \mathrm{H} & -2.5463493 & 1.6051597 & -1.3165447 \\ \mathrm{C} & 0.0392489 & 2.5082554 & -2.2479555 \\ \mathrm{H} & 1.1242684 & 2.6692890 & -2.2311235 \\ \mathrm{H} & -0.2476916 & 2.2578135 & -3.2832126 \\ \mathrm{H} & -0.4677012 & 3.4484489 & -1.9895954 \\ & & & \end{array}$

\section{TS $4+C$ (exo)}

Imaginary Frequencies $=1(-380.18 \mathrm{~cm}-1)$

Energy (BP86/def-TZVP, level of optimization) $=-1014.0981328167 \mathrm{H}$ Energy (M0 6-2X/def2-TZVP) $=-1013.629656179 \mathrm{H}$

$\begin{array}{lrrr}\text { C } & -0.8375464 & 0.7801997 & -2.0980318 \\ \text { C } & -0.0755223 & 0.7840978 & -3.2639664 \\ \text { C } & 1.3166596 & 0.8836772 & -3.2420429 \\ \mathrm{C} & 2.0447477 & 1.0060631 & -2.0426798 \\ \mathrm{H} & -0.5770009 & 0.5613907 & -4.2087911 \\ \mathrm{H} & 1.8661720 & 0.7091606 & -4.1711595 \\ \mathrm{C} & 0.2579672 & -1.2481052 & -1.1942328 \\ \mathrm{C} & 1.5749659 & -0.7814083 & -1.0094033 \\ \mathrm{C} & -0.4697812 & -1.1065505 & 0.0907900 \\ \mathrm{H} & 2.4303101 & -1.2706493 & -1.4726027 \\ \mathrm{C} & 1.6991992 & -0.3415701 & 0.4190764 \\ \mathrm{O} & 2.7004378 & 0.0470598 & 1.0078537 \\ \mathrm{O} & -1.5845440 & -1.5179678 & 0.3862912\end{array}$




$\begin{array}{rrrr}\text { N } & 0.4149012 & -0.4515602 & 0.9963549 \\ \mathrm{C} & 0.0675583 & -0.0761661 & 2.3294659 \\ \mathrm{C} & -1.0657959 & 0.7208829 & 2.5447738 \\ \mathrm{C} & -1.4200323 & 1.0787881 & 3.8484377 \\ \mathrm{C} & -0.6405422 & 0.6577613 & 4.9325436 \\ \mathrm{C} & 0.4923692 & -0.1334895 & 4.7095672 \\ \mathrm{C} & 0.8464053 & -0.5117000 & 3.4106910 \\ \mathrm{H} & -1.6635465 & 1.0561725 & 1.6966102 \\ \mathrm{H} & -2.3048564 & 1.6952184 & 4.0153265 \\ \mathrm{H} & 1.1014190 & -0.4686648 & 5.5507441 \\ \mathrm{H} & 1.7175224 & -1.1423077 & 3.2366436 \\ \mathrm{H} & -0.9162607 & 0.9445233 & 5.9486461 \\ \mathrm{C} & -2.2886504 & 0.5525162 & -2.1523320 \\ \mathrm{H} & -0.4708567 & 1.2616518 & -1.1923107 \\ \mathrm{H} & 1.5667430 & 1.5762402 & -1.2398874 \\ \mathrm{C} & 3.5458167 & 1.0823286 & -2.0565298 \\ \mathrm{H} & 3.9701323 & 0.8103675 & -1.0811848 \\ \mathrm{H} & 3.9781951 & 0.4369502 & -2.8335050 \\ \mathrm{H} & 3.8519111 & 2.1206754 & -2.2659936 \\ \mathrm{O} & -2.9003556 & 0.0216151 & -3.0777336 \\ \mathrm{O} & -2.9088231 & 1.0013985 & -1.0259354 \\ \mathrm{H} & -3.8652567 & 0.7969413 & -1.1080083 \\ \mathrm{C} & -0.1670428 & -2.2258798 & -2.2361374 \\ \mathrm{H} & -1.2587353 & -2.3209493 & -2.2873617 \\ \mathrm{H} & 0.2188913 & -1.9296608 & -3.2225878 \\ \mathrm{H} & 0.2538647 & -3.2206925 & -2.0122244 \\ & & & \end{array}$

\section{Product $4+\mathrm{C}$ (endo)}

Imaginary Frequencies $=0$

Energy(BP86/def-TZVP, level of optimization) $=-1014.1557802551 \mathrm{H}$ Energy (M06-2X/def2-TZVP) $=-1013.709804084 \mathrm{H}$

$\begin{array}{llll}\text { C } & -2.0423088 & -0.3155627 & 1.3955357\end{array}$

C $\quad-3.3661066 \quad-0.4293542 \quad 1.5690994$

C $\quad-4.2670650 \quad-0.2976017 \quad 0.3658625$

$\mathrm{H} \quad-1.3280108 \quad-0.4336145 \quad 2.2110319$

$\mathrm{H} \quad-3.7983064 \quad-0.6448269 \quad 2.5493511$

C $\quad-2.1877568 \quad-0.8763417 \quad-1.1126029$

C $\quad-3.6728651-1.1910353 \quad-0.7632017$

$\begin{array}{llll}\text { C } & -1.5061467 & -2.2494000 & -1.1292868\end{array}$

$\begin{array}{llll}\text { C } & -3.6992185 & -2.6818219 & -0.4386601\end{array}$

$\begin{array}{llll}0 & -4.6455473 & -3.3532564 & -0.0582293\end{array}$

$\begin{array}{llll}0 & -0.3599641 & -2.4878225 & -1.4796292\end{array}$

$\begin{array}{llll}\mathrm{N} & -2.4198171 & -3.2161565 & -0.6927588\end{array}$

$\begin{array}{llll}\mathrm{N} & -2.4198171 & -3.2161565 & -0.6927588 \\ \mathrm{C} & -2.1030790 & -4.6123733 & -0.5724692\end{array}$

C $\quad-2.7516592 \quad-5.5402834 \quad-1.3950324$

C $\quad-2.4415491 \quad-6.8979297 \quad-1.2709222$

$\begin{array}{llll}\text { C } & -1.4861156 & -7.3202470 & -0.3391015\end{array}$

$\begin{array}{llll}\text { C } & -0.8399767 & -6.3828408 & 0.4752385\end{array}$

$\begin{array}{llll}\text { C } & -1.1492101 & -5.0237279 & 0.3647621\end{array}$

$\mathrm{H} \quad-3.4866574 \quad-5.2025800 \quad-2.1263408$

$\mathrm{H} \quad-2.9456480 \quad-7.6257719 \quad-1.9085494$

$\mathrm{H} \quad-0.0954757 \quad-6.7081800 \quad 1.2034804$ 


$\begin{array}{lrrr}\mathrm{H} & -0.6575890 & -4.2883262 & 1.0023863 \\ \mathrm{H} & -1.2449551 & -8.3804981 & -0.2476037 \\ \mathrm{C} & 0.0017928 & 0.0307195 & -0.0156311 \\ \mathrm{H} & -1.8444399 & 1.0502783 & -0.2391336 \\ \mathrm{H} & -4.1738128 & 0.7352493 & -0.0217652 \\ \mathrm{C} & -5.7507452 & -0.5391909 & 0.6607413 \\ \mathrm{H} & -6.3534205 & -0.4478523 & -0.2541344 \\ \mathrm{H} & -5.9222854 & -1.5365934 & 1.0845767 \\ \mathrm{H} & -6.1100375 & 0.2110183 & 1.3801883 \\ \mathrm{O} & 0.7222705 & -0.5821146 & 0.7563977 \\ \mathrm{O} & 0.4865731 & 0.8460367 & -0.9828089 \\ \mathrm{H} & 1.4675388 & 0.7985115 & -0.9556429 \\ \mathrm{H} & -4.2895909 & -1.0641031 & -1.6675993 \\ \mathrm{C} & -2.0508760 & -0.2201558 & -2.4961869 \\ \mathrm{H} & -0.9986975 & -0.0617985 & -2.7597279 \\ \mathrm{H} & -2.5136778 & -0.8529139 & -3.2666104 \\ \mathrm{H} & -2.5631105 & 0.7523750 & -2.4970043\end{array}$

\section{Product $4+C$ (exo)}

Imaginary Frequencies $=0$

Energy (BP86/def-TZVP, level of optimization) $=-1014.1565415575 \mathrm{H}$

Energy (M06-2X/def2-TZVP) $=-1013.710018237 \mathrm{H}$

$\begin{array}{lrrr}\text { C } & 1.4654984 & 0.3069843 & 0.2589146 \\ \mathrm{C} & 2.2312390 & 0.5400672 & -1.0235906 \\ \mathrm{C} & 3.5535499 & 0.7316836 & -0.9528580 \\ \mathrm{C} & 4.2723380 & 0.7295988 & 0.3747631 \\ \mathrm{H} & 1.6912825 & 0.5398360 & -1.9717516 \\ \mathrm{H} & 4.1415821 & 0.9156490 & -1.8558207 \\ \mathrm{C} & 2.2120520 & -0.8194350 & 1.0819130 \\ \mathrm{C} & 3.6740354 & -0.3516897 & 1.3320881 \\ \mathrm{C} & 1.6013833 & -0.8885367 & 2.4866010 \\ \mathrm{H} & 4.3231836 & -1.2415348 & 1.2740191 \\ \mathrm{C} & 3.7058122 & 0.0846288 & 2.7909166 \\ \mathrm{O} & 4.6080333 & 0.6626199 & 3.3767282 \\ \mathrm{O} & 0.5197685 & -1.3685187 & 2.7891989 \\ \mathrm{~N} & 2.4934283 & -0.3000834 & 3.3961450 \\ \mathrm{C} & 2.2111124 & -0.1350106 & 4.7947026 \\ \mathrm{C} & 1.1447188 & 0.6794465 & 5.1915196 \\ \mathrm{C} & 0.8686329 & 0.8301899 & 6.5538050 \\ \mathrm{C} & 1.6584208 & 0.1787693 & 7.5085183 \\ \mathrm{C} & 2.7259473 & -0.6297534 & 7.1006241 \\ \mathrm{C} & 3.0044301 & -0.7944849 & 5.7404715 \\ \mathrm{H} & 0.5408594 & 1.1928038 & 4.4425826 \\ \mathrm{H} & 0.0368976 & 1.4626075 & 6.8679070 \\ \mathrm{H} & 3.3422526 & -1.1405052 & 7.8420048 \\ \mathrm{H} & 3.8263822 & -1.4326248 & 5.4145242 \\ \mathrm{H} & 1.4421344 & 0.3014693 & 8.5709835 \\ \mathrm{C} & -0.0001587 & -0.0027551 & 0.0002606 \\ \mathrm{H} & 1.5001358 & 1.2222330 & 0.8737614 \\ \mathrm{H} & 4.0903014 & 1.7148525 & 0.8446481 \\ \mathrm{C} & 5.7902214 & 0.5620844 & 0.2184104 \\ \mathrm{H} & 6.2916000 & 0.5992797 & 1.1942746 \\ \mathrm{H} & 6.0280313 & -0.4020936 & -0.2569075\end{array}$




$\begin{array}{rrrr}\mathrm{H} & 6.2012917 & 1.3646176 & -0.4109711 \\ \mathrm{O} & -0.4167618 & -0.7882613 & -0.8377952 \\ \mathrm{O} & -0.8145411 & 0.7310886 & 0.7943140 \\ \mathrm{H} & -1.7438000 & 0.4873535 & 0.5876557 \\ \mathrm{C} & 2.1326539 & -2.1990882 & 0.4136399 \\ \mathrm{H} & 1.0965522 & -2.5530578 & 0.3512300 \\ \mathrm{H} & 2.5505769 & -2.1519170 & -0.6001600 \\ \mathrm{H} & 2.7153615 & -2.9289169 & 0.9933308\end{array}$

\section{TS $5+C$ (endo)}

Imaginary Frequencies $=1(-365.09 \mathrm{~cm}-1)$

Energy (BP86/def-TZVP, level of optimization) $=-1355.0502523713 \mathrm{H}$

Energy (M06-2X/def2-TZVP) $=-1354.419826559 \mathrm{H}$

$\begin{array}{lrrr}\mathrm{C} & -1.0398211 & 1.3882125 & 0.5081672 \\ \mathrm{C} & -0.6404754 & 0.7611024 & -0.7060653 \\ \mathrm{C} & 0.7291622 & 0.6670614 & -0.6745122 \\ \mathrm{C} & 1.1413180 & 1.1654398 & 0.6165551 \\ \mathrm{O} & 0.0737360 & 1.9386726 & 1.1043669 \\ \mathrm{H} & -1.3226512 & 0.3850539 & -1.4635308 \\ \mathrm{H} & 1.3833362 & 0.1792184 & -1.3918320 \\ \mathrm{C} & -2.3659054 & 2.0038564 & 0.8427158 \\ \mathrm{H} & -2.4127864 & 2.2487852 & 1.9104691 \\ \mathrm{H} & -3.1602627 & 1.2836204 & 0.6087702 \\ \mathrm{C} & 2.5140186 & 1.6808216 & 0.9718949 \\ \mathrm{H} & 2.5417099 & 1.9503683 & 2.0367880 \\ \mathrm{H} & 3.2436500 & 0.8790471 & 0.7956113 \\ \mathrm{~N} & -2.6301046 & 3.2180113 & 0.0717764 \\ \mathrm{~N} & 2.9196808 & 2.8286413 & 0.1713986 \\ \mathrm{C} & -3.3960262 & 3.2270909 & -1.0549201 \\ \mathrm{C} & 3.8281624 & 2.7475992 & -0.8416389 \\ \mathrm{C} & 4.1274412 & 4.0471453 & -1.5589590 \\ \mathrm{H} & 3.8989589 & 3.9229733 & -2.6266559 \\ \mathrm{H} & 3.5591983 & 4.9003752 & -1.1678182 \\ \mathrm{H} & 5.2018308 & 4.2610409 & -1.4721373 \\ \mathrm{O} & 4.3821128 & 1.6790602 & -1.1549438 \\ \mathrm{H} & 2.4987971 & 3.7303908 & 0.3787925 \\ \mathrm{C} & -3.5735834 & 4.5770576 & -1.7173781 \\ \mathrm{H} & -3.0416706 & 5.3869704 & -1.2026701 \\ \mathrm{H} & -3.2140551 & 4.5131012 & -2.7537501 \\ \mathrm{H} & -4.6455498 & 4.8166057 & -1.7515274 \\ \mathrm{O} & -3.9247427 & 2.1954496 & -1.5057530 \\ \mathrm{H} & -2.2118654 & 4.0882055 & 0.3899721 \\ \mathrm{C} & 0.7724700 & -0.3745885 & 1.7824905 \\ \mathrm{C} & -0.6447121 & -0.4251581 & 1.9497680 \\ \mathrm{H} & 1.4277381 & -0.0923623 & 2.6090824 \\ \mathrm{C} & 1.1794044 & -1.5831843 & 0.9972070 \\ \mathrm{C} & -1.1425146 & -1.5889637 & 1.1940486 \\ \mathrm{O} & -2.2864767 & -2.0275659 & 1.1112104 \\ \mathrm{O} & 2.3141955 & -1.9684047 & 0.7382147 \\ \mathrm{~N} & -0.0091853 & -2.2095119 & 0.5753674 \\ \mathrm{C} & -0.0808724 & -3.3520581 & -0.2761428 \\ \mathrm{C} & 0.6816636 & -4.4921352 & 0.0153076 \\ \mathrm{C} & 0.6068626 & -5.6056617 & -0.8268286\end{array}$




$\begin{array}{lrrr}\text { C } & -0.2358543 & -5.5920989 & -1.9442475 \\ \text { C } & -1.0031715 & -4.4548674 & -2.2231302 \\ \text { C } & -0.9241488 & -3.3309763 & -1.3961277 \\ \text { H } & 1.3239663 & -4.5066066 & 0.8953601 \\ \text { H } & 1.2042178 & -6.4905977 & -0.6010532 \\ \text { H } & -1.6625857 & -4.4374789 & -3.0925017 \\ \text { H } & -1.5120768 & -2.4407443 & -1.6190010 \\ \text { H } & -0.2955478 & -6.4657341 & -2.5954198 \\ \mathrm{C} & -1.3794801 & 0.0431926 & 3.1750314 \\ \text { H } & -2.4657676 & 0.0601804 & 3.0155269 \\ \text { H } & -1.1829971 & -0.6375193 & 4.0202162 \\ \text { H } & -1.0470033 & 1.0445174 & 3.4794027\end{array}$

TS $5+C$ (exo)

Imaginary Frequencies $=1(-367.73 \mathrm{~cm}-1)$

Energy (BP86/def-TZVP, level of optimization) $=-1355.0495527046 \mathrm{H}$

Energy (M06-2X/def2-TZVP) $=-1354.422799540 \mathrm{H}$

C

C $\quad 0.8063260$

1.0983325

0.3726463

2.1887696

1. 2551755

C $\quad-0.5577535$

2. 2767785

$-1.1065870$

1. 2107829

$-0.0919166$

0.7837420

1.3699663

1.5605231

2.7564753

$-1.1375911$

2. 3579277

2. 2765954

2.9175494

0.7687328

$-0.2054713$

0.7139748

1. 1827631

0.2242479

1. 2943055

1. 7811409

2.2674647

2. 7056589

3. 4024907

4.4158323

0.5712174

$-0.2916987$

1. 7930122

2.0285507

$-0.3339645$

$-0.8327159$

0.4013555

$-0.0047047$

$-0.5012053$

0.8157861

$-1.3204180$

$-0.9524712$

3.7091443

$-3.4183869$

$-3.5653172$

5.3629068

$-1.1057018$

$-0.6325869$

$-1.7482046$

$-1.4277765$

$-3.1021392$

4.0956692

$-2.6898990$

$-4.6344013$

4.6035577

$-1.9188993$

3. 5951890

0.4995070

$-3.8966749$

2. 1710544

$-1.8930844$

$-2.3634489$

3. 6723739

$-2.2444874$

3. 3111596

3. 4979541

$-3.1128683$

3.8009023

4.6968800

$-1.8794668$

5.0089600

3.5891023

$-2.5565903$

4. 3615412

2.7517367

$-0.0474711$

2. 2279210

1.7977138

$-2.2032201$

1.9799237

$-0.8363905$

$-0.4663663$

1.8665273

0.9835536

$-0.3361490$

1.1971582

$-1.4646128$

$-1.6577610$

2.6960035

$-1.3281009$

$-1.5078212$

1.0767127

$-1.8569652$

0.8747812

2.0994065

$-2.1557360$

1. 0748212 


$\begin{array}{lrrr}\text { N } & -0.1933280 & -2.1901778 & 0.5976511 \\ \mathrm{C} & -0.2295559 & -3.3058594 & -0.2927863 \\ \mathrm{C} & 0.3364296 & -4.5284366 & 0.0937588 \\ \mathrm{C} & 0.3005270 & -5.6133182 & -0.7879041 \\ \mathrm{C} & -0.3097662 & -5.4856048 & -2.0411105 \\ \mathrm{C} & -0.8804649 & -4.2636977 & -2.4174446 \\ \mathrm{C} & -0.8353981 & -3.1692417 & -1.5492491 \\ \mathrm{H} & 0.7964144 & -4.6276748 & 1.0766818 \\ \mathrm{H} & 0.7443091 & -6.5642467 & -0.4886140 \\ \mathrm{H} & -1.3559352 & -4.1574408 & -3.3939130 \\ \mathrm{H} & -1.2630997 & -2.2111205 & -1.8456544 \\ \mathrm{H} & -0.3405310 & -6.3366296 & -2.7234626 \\ \mathrm{C} & 1.3895843 & -0.0887745 & 3.1919711 \\ \mathrm{H} & 2.4613290 & -0.0197187 & 2.9609357 \\ \mathrm{H} & 1.0548195 & 0.8714016 & 3.6031877 \\ \mathrm{H} & 1.2709452 & -0.8484332 & 3.9826585\end{array}$

\section{Product $5+\mathrm{C}$ (endo)}

Imaginary Frequencies $=0$

Energy (BP86/def-TZVP, level of optimization) $=-1355.0803295156 \mathrm{H}$ Energy (M06-2X/def2-TZVP) $=-1354.469057918 \mathrm{H}$

$\begin{array}{lrrr}\mathrm{C} & -1.0113157 & 1.4767601 & 0.6713672 \\ \mathrm{C} & -0.6412134 & 1.1133627 & -0.7652551 \\ \mathrm{C} & 0.6967269 & 1.0728848 & -0.8082084 \\ \mathrm{C} & 1.1690598 & 1.3991875 & 0.6060814 \\ \mathrm{O} & 0.1222291 & 2.3018529 & 1.0663443 \\ \mathrm{H} & -1.3561629 & 0.8642849 & -1.5470310 \\ \mathrm{H} & 1.3447627 & 0.7762208 & -1.6307187 \\ \mathrm{C} & -2.3326707 & 2.1901993 & 0.8970964 \\ \mathrm{H} & -2.4147784 & 2.5068970 & 1.9458351 \\ \mathrm{H} & -3.1587858 & 1.5017350 & 0.6753566 \\ \mathrm{C} & 2.5524866 & 1.9886041 & 0.8067573 \\ \mathrm{H} & 2.6881212 & 2.2397067 & 1.8700754 \\ \mathrm{H} & 3.3079405 & 1.2409930 & 0.5301367 \\ \mathrm{~N} & -2.4842716 & 3.3470079 & 0.0270714 \\ \mathrm{~N} & 2.7808559 & 3.1664743 & -0.0143482 \\ \mathrm{C} & -3.4027012 & 3.4187729 & -0.9757477 \\ \mathrm{C} & 3.6841006 & 3.2095120 & -1.0332385 \\ \mathrm{C} & 3.7837375 & 4.5309288 & -1.7665061 \\ \mathrm{H} & 3.5674651 & 4.3633931 & -2.8307839 \\ \mathrm{H} & 3.1001522 & 5.2963466 & -1.3780030 \\ \mathrm{H} & 4.8160679 & 4.9005652 & -1.6923851 \\ \mathrm{O} & 4.3887680 & 2.2318394 & -1.3431881 \\ \mathrm{H} & 2.2374491 & 4.0011428 & 0.1894107 \\ \mathrm{C} & -3.4068215 & 4.7083390 & -1.7704726 \\ \mathrm{H} & -2.6640189 & 5.4365270 & -1.4210258 \\ \mathrm{H} & -3.2101725 & 4.4746962 & -2.8262165 \\ \mathrm{H} & -4.4073739 & 5.1588503 & -1.7114522 \\ \mathrm{O} & -4.1929942 & 2.4903234 & -1.2262372 \\ \mathrm{H} & -1.8660684 & 4.1397134 & 0.1787819 \\ \mathrm{C} & 0.8293022 & 0.1429403 & 1.5124936 \\ \mathrm{C} & -0.7155266 & 0.1885925 & 1.5813700 \\ \mathrm{H} & 1.3101566 & 0.2581311 & 2.4933380\end{array}$




$\begin{array}{rrrr}\text { C } & 1.1839055 & -1.2038071 & 0.9207056 \\ \mathrm{C} & -1.1633117 & -1.1182978 & 0.9344026 \\ \mathrm{O} & -2.3109005 & -1.4858429 & 0.7339362 \\ \mathrm{O} & 2.3002649 & -1.6558603 & 0.7149225 \\ \mathrm{~N} & -0.0176844 & -1.8772506 & 0.6192928 \\ \mathrm{C} & -0.0670777 & -3.1777139 & 0.0109720 \\ \mathrm{C} & 0.4412894 & -4.2797049 & 0.7078273 \\ \mathrm{C} & 0.3979270 & -5.5443109 & 0.1133599 \\ \mathrm{C} & -0.1576696 & -5.7044628 & -1.1615205 \\ \mathrm{C} & -0.6680819 & -4.5960623 & -1.8474167 \\ \mathrm{C} & -0.6205052 & -3.3255212 & -1.2656221 \\ \mathrm{H} & 0.8611524 & -4.1483822 & 1.7056531 \\ \mathrm{H} & 0.7946873 & -6.4063225 & 0.6517921 \\ \mathrm{H} & -1.1008101 & -4.7169024 & -2.8416420 \\ \mathrm{H} & -1.0062764 & -2.4563908 & -1.7990727 \\ \mathrm{H} & -0.1936392 & -6.6937851 & -1.6205824 \\ \mathrm{C} & -1.2790231 & 0.3007969 & 3.0036085 \\ \mathrm{H} & -2.3761328 & 0.2547964 & 2.9996040 \\ \mathrm{H} & -0.9061510 & -0.5227838 & 3.6292364 \\ \mathrm{H} & -0.9605589 & 1.2467834 & 3.4617557\end{array}$

\section{Product $5+C$ (exo)}

Imaginary Frequencies $=0$

Energy (BP86/def-TZVP, level of optimization) $=-1355.0817102293 \mathrm{H}$ Energy (M0 6-2X/def2-TZVP) $=-1354.472634196 \mathrm{H}$

$\begin{array}{lrrr}\mathrm{C} & 1.1530675 & 1.2617185 & 0.3895817 \\ \mathrm{C} & 0.7897737 & 2.5515336 & 1.1235209 \\ \mathrm{C} & -0.5465773 & 2.5887847 & 1.1677171 \\ \mathrm{C} & -1.0281428 & 1.3236864 & 0.4619162 \\ \mathrm{O} & 0.0242267 & 1.1077732 & -0.5197615 \\ \mathrm{H} & 1.5061579 & 3.2299857 & 1.5820434 \\ \mathrm{H} & -1.1974774 & 3.2960890 & 1.6783126 \\ \mathrm{C} & 2.4741061 & 1.2335359 & -0.3614663 \\ \mathrm{H} & 2.5524561 & 0.3131803 & -0.9553413 \\ \mathrm{H} & 3.3076927 & 1.2507012 & 0.3535550 \\ \mathrm{C} & -2.4007596 & 1.3517883 & -0.1879471 \\ \mathrm{H} & -2.5727767 & 0.4222117 & -0.7486083 \\ \mathrm{H} & -3.1723453 & 1.4297523 & 0.5895591 \\ \mathrm{~N} & 2.6240487 & 2.3925588 & -1.2301718 \\ \mathrm{~N} & -2.5555574 & 2.4940421 & -1.0762920 \\ \mathrm{C} & 3.5575615 & 3.3643642 & -1.0384934 \\ \mathrm{C} & -3.4180003 & 3.5209726 & -0.8418815 \\ \mathrm{C} & -3.4319416 & 4.6158961 & -1.8880230 \\ \mathrm{H} & -3.1669716 & 5.5683064 & -1.4078656 \\ \mathrm{H} & -2.7411655 & 4.4303565 & -2.7200302 \\ \mathrm{H} & -4.4522127 & 4.7167694 & -2.2832449 \\ \mathrm{O} & -4.1528470 & 3.5629743 & 0.1619505 \\ \mathrm{H} & -1.9830770 & 2.5231039 & -1.9155821 \\ \mathrm{C} & 3.5541096 & 4.4856375 & -2.0566599 \\ \mathrm{H} & 2.7823247 & 4.3691743 & -2.8279303 \\ \mathrm{H} & 3.3975870 & 5.4394025 & -1.5336558 \\ \mathrm{H} & 4.5402372 & 4.5303512 & -2.5395550 \\ \mathrm{O} & 4.3677222 & 3.3380767 & -0.0936126\end{array}$




$\begin{array}{rrrr}\mathrm{H} & 1.9924992 & 2.4755917 & -2.0221453 \\ \mathrm{C} & 0.8414477 & 0.0757787 & 1.4150160 \\ \mathrm{C} & -0.7122069 & 0.1473729 & 1.4808239 \\ \mathrm{C} & 1.1177212 & -1.2852416 & 0.7673669 \\ \mathrm{H} & -1.1240530 & 0.3573123 & 2.4753605 \\ \mathrm{C} & -1.2089405 & -1.2042624 & 1.0057003 \\ \mathrm{O} & -2.3611841 & -1.6120444 & 1.0016502 \\ \mathrm{O} & 2.2099302 & -1.7595507 & 0.4940075 \\ \mathrm{~N} & -0.1032632 & -1.9500327 & 0.5614676 \\ \mathrm{C} & -0.2069389 & -3.2671064 & -0.0047777 \\ \mathrm{C} & 0.3125991 & -4.3622896 & 0.6936838 \\ \mathrm{C} & 0.2127583 & -5.6395068 & 0.1335676 \\ \mathrm{C} & -0.4074459 & -5.8171092 & -1.1087759 \\ \mathrm{C} & -0.9276339 & -4.7144512 & -1.7966946 \\ \mathrm{C} & -0.8254402 & -3.4322437 & -1.2487998 \\ \mathrm{H} & 0.7844415 & -4.2169503 & 1.6660913 \\ \mathrm{H} & 0.6174857 & -6.4972320 & 0.6728645 \\ \mathrm{H} & -1.4101810 & -4.8491125 & -2.7658544 \\ \mathrm{H} & -1.2172717 & -2.5663389 & -1.7835106 \\ \mathrm{H} & -0.4856035 & -6.8158483 & -1.5413235 \\ \mathrm{C} & 1.5814074 & 0.1700692 & 2.7531076 \\ \mathrm{H} & 2.6669371 & 0.0953017 & 2.6028411 \\ \mathrm{H} & 1.3559953 & 1.1250391 & 3.2457916 \\ \mathrm{H} & 1.2751407 & -0.6418818 & 3.4277673\end{array}$

TS $1+\mathrm{D}$

Imaginary Frequencies $=1(-388.93 \mathrm{~cm}-1)$

Energy (BP86/def-TZVP, level of optimization) $=-1475.1826705577 \mathrm{H}$

Energy (M0 6-2X/def2-TZVP) $=-1474.539550097 \mathrm{H}$

$\begin{array}{llll}\text { C } & 0.2835064 & -3.8736978 & 0.2676415\end{array}$

$\begin{array}{llll}\text { C } & 0.7175044 & -3.6974434 & -1.0806714\end{array}$

C $\quad 0.3132787 \quad-2.4826118 \quad-1.7470954$

C $\quad-0.7067020 \quad-2.6274999 \quad 2.2830139$

$\mathrm{H} \quad 0.6732441 \quad-2.3320303 \quad-2.7686301$

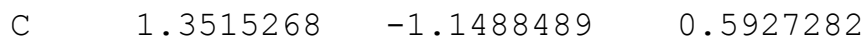

C $\quad 1.5875695-1.1085168 \quad-0.7896723$

$\begin{array}{llll}\text { C } & 0.9070485 & 0.0882092 & 1.3185175\end{array}$

$\mathrm{H} \quad 1.8439453 \quad-1.9103928 \quad 1.1954823$

$\mathrm{H} \quad 2.4190229 \quad-1.7148639 \quad-1.1538689$

$\begin{array}{llll}\text { C } & 1.4516560 & 0.2266204 & -1.4992535\end{array}$

$\begin{array}{llll}\mathrm{H} & 2.3933320 & 0.7758542 & -1.3217561\end{array}$

$\begin{array}{llll}\text { C } & -1.4656239 & -2.1560115 & -0.0934221\end{array}$

$\begin{array}{llll}\text { C } & -1.0094378 & -2.0038053 & -1.4406198\end{array}$

C $\quad \begin{array}{rrr}-1.0094378 & -2.0038053 & -1.4406198\end{array}$

C $\quad 1.9450486-5.7916065-1.0065838$

C $\quad 1.4930399 \quad-5.9931541 \quad 0.3133541$

$\begin{array}{llll}\text { C } & 0.6922044 & -5.0500671 & 0.9453561\end{array}$

C $\quad-2.7493595 \quad-1.6444571 \quad 0.2322337$

$\begin{array}{llll}\text { C } & -3.5384792 & -1.0373858 & -0.7367944\end{array}$

$\begin{array}{llll}\text { C } & -3.0785853 & -0.8897886 & -2.0626399\end{array}$

$\begin{array}{llll}\text { C } & -1.8196461 & -1.3599816 & -2.4047577\end{array}$

$\mathrm{H} \quad 1.8983752 \quad-4.4769453 \quad-2.7186226$ 


$\begin{array}{rrrr}\mathrm{H} & 2.5795458 & -6.5372824 & -1.4886897 \\ \mathrm{H} & 1.7703409 & -6.9039306 & 0.8477865 \\ \mathrm{H} & 0.3594474 & -5.2305894 & 1.9652287 \\ \mathrm{H} & -3.1191927 & -1.7454073 & 1.2492699 \\ \mathrm{H} & -4.5343876 & -0.6800955 & -0.4673567 \\ \mathrm{H} & -3.7121442 & -0.4151166 & -2.8136697 \\ \mathrm{H} & -1.4465151 & -1.2579576 & -3.4261776 \\ \mathrm{O} & 0.3097442 & -3.1745293 & 3.0179412 \\ \mathrm{O} & -1.5896089 & -1.9736925 & 2.8433933 \\ \mathrm{H} & 0.4661713 & -0.1430207 & 2.2982674 \\ \mathrm{H} & 0.1217637 & -2.9571672 & 3.9562212 \\ \mathrm{H} & 1.8225512 & 0.6739870 & 1.5373438 \\ \mathrm{H} & 1.3807933 & 0.0934087 & -2.5870006 \\ \mathrm{C} & 0.2631693 & 1.0789589 & -1.0029874 \\ \mathrm{H} & -0.6277979 & 0.8152228 & -1.5921457 \\ \mathrm{C} & 0.4940592 & 2.5627059 & -1.2380466 \\ \mathrm{C} & -0.0742956 & 0.9552204 & 0.4991721 \\ \mathrm{C} & -0.1126846 & 2.3835862 & 1.0189436 \\ \mathrm{H} & -1.0847174 & 0.5419822 & 0.6269677 \\ \mathrm{O} & 0.8495908 & 3.1033370 & -2.2749518 \\ \mathrm{O} & -0.3847847 & 2.7515731 & 2.1525451 \\ \mathrm{~N} & 0.2291606 & 3.2494467 & -0.0367301 \\ \mathrm{C} & 0.3020601 & 4.6770392 & 0.0942694 \\ \mathrm{C} & 1.2316929 & 5.2444013 & 0.9730797 \\ \mathrm{C} & 1.2943939 & 6.6355695 & 1.0984898 \\ \mathrm{C} & 0.4416820 & 7.4502770 & 0.3443334 \\ \mathrm{C} & -0.4813540 & 6.8725871 & -0.5354646 \\ \mathrm{C} & -0.5585474 & 5.4822359 & -0.6607088 \\ \mathrm{H} & 1.9016666 & 4.6044065 & 1.5480525 \\ \mathrm{H} & 2.0162078 & 7.0824509 & 1.7838061 \\ \mathrm{H} & 0.4965287 & 8.5356985 & 0.4421747 \\ \mathrm{H} & -1.1488451 & 7.5039894 & -1.1239143 \\ \mathrm{H} & -1.2830287 & 5.0241946 & -1.3348341 \\ & & & \\ & & & \end{array}$

\section{Product $1+D$}

Imaginary Frequencies $=0$

Energy (BP86/def-TZVP, level of optimization) $=-1475.2387539299 \mathrm{H}$

Energy (M06-2X/def2-TZVP) $=-1474.615942398 \mathrm{H}$

$\begin{array}{lrrr}\text { C } & 0.3736804 & 0.2793585 & -1.4529259 \\ \text { C } & 0.9619665 & -1.0100362 & -2.0683338 \\ \text { C } & 1.2976643 & -0.8668821 & -3.4274151 \\ \text { C } & 1.0298840 & 0.5190243 & -3.9742706 \\ \text { C } & 0.0083348 & -0.0024092 & 0.0032143 \\ \text { H } & 1.2766031 & 0.5977481 & -5.0412727 \\ \text { C } & 1.5054280 & 1.3857209 & -1.6154979 \\ \text { C } & 1.9406292 & 1.4718646 & -3.1194556 \\ \text { C } & 1.1512534 & 2.7679135 & -1.0200338 \\ \text { H } & 2.3594602 & 1.0143906 & -1.0357328 \\ \text { H } & 2.9554946 & 1.0566838 & -3.2029030 \\ \text { C } & 2.0373077 & 2.9173065 & -3.6430966 \\ \text { H } & 2.9070075 & 3.3922142 & -3.1617926 \\ \mathrm{C} & -0.8068576 & 0.6788019 & -2.3486372 \\ \text { C } & -0.4280488 & 0.8171541 & -3.7010518\end{array}$




$\begin{array}{rrrr}\mathrm{C} & 1.8531649 & -1.9359824 & -4.1321897 \\ \mathrm{C} & 2.0676782 & -3.1587218 & -3.4805614 \\ \mathrm{C} & 1.7338837 & -3.3016063 & -2.1299171 \\ \mathrm{C} & 1.1822505 & -2.2261213 & -1.4175378 \\ \mathrm{C} & -2.1315285 & 0.9301541 & -1.9729864 \\ \mathrm{C} & -3.0707277 & 1.3032289 & -2.9448918 \\ \mathrm{C} & -2.6960795 & 1.4252620 & -4.2877800 \\ \mathrm{C} & -1.3699110 & 1.1818621 & -4.6668002 \\ \mathrm{H} & 2.1152838 & -1.8176282 & -5.1861144 \\ \mathrm{H} & 2.4946476 & -3.9999177 & -4.0297036 \\ \mathrm{H} & 1.8992798 & -4.2540616 & -1.6231910 \\ \mathrm{H} & 0.9280579 & -2.3578691 & -0.3638832 \\ \mathrm{H} & -2.4398300 & 0.8305227 & -0.9338052 \\ \mathrm{H} & -4.1034461 & 1.4937585 & -2.6471762 \\ \mathrm{H} & -3.4352775 & 1.7071939 & -5.0399920 \\ \mathrm{H} & -1.0675690 & 1.2777596 & -5.7122361 \\ \mathrm{O} & 1.0202500 & 0.2746684 & 0.8587206 \\ \mathrm{O} & -1.0450272 & -0.4935595 & 0.3803231 \\ \mathrm{H} & 0.6750935 & 2.6579751 & -0.0367315 \\ \mathrm{H} & 0.7276368 & 0.0160478 & 1.7605690 \\ \mathrm{H} & 2.1032023 & 3.2912601 & -0.8347006 \\ \mathrm{H} & 2.2498008 & 2.9070384 & -4.7216668 \\ \mathrm{C} & 0.7828543 & 3.7692731 & -3.3703815 \\ \mathrm{H} & -0.0095822 & 3.4922356 & -4.0806012 \\ \mathrm{C} & 1.0540494 & 5.2497773 & -3.5828759 \\ \mathrm{C} & 0.2656353 & 3.6547389 & -1.9202073 \\ \mathrm{C} & 0.2220799 & 5.0814729 & -1.3979033 \\ \mathrm{H} & -0.7671854 & 3.2804847 & -1.9035180 \\ \mathrm{O} & 1.5088485 & 5.7867394 & -4.5829430 \\ \mathrm{O} & -0.1528587 & 5.4580432 & -0.2962370 \\ \mathrm{~N} & 0.6996705 & 5.9396919 & -2.4066843 \\ \mathrm{C} & 0.8040187 & 7.3633578 & -2.2545927 \\ \mathrm{C} & 1.6897222 & 7.8925819 & -1.3092055 \\ \mathrm{C} & 1.7821072 & 9.2795483 & -1.1585512 \\ \mathrm{C} & 1.0017594 & 10.1277803 & -1.9529798 \\ \mathrm{C} & 0.1223786 & 9.5882017 & -2.8990863 \\ \mathrm{C} & 0.0163433 & 8.2022583 & -3.0509610 \\ \mathrm{H} & 2.3023277 & 7.2257404 & -0.7015067 \\ \mathrm{H} & 2.4696327 & 9.6966752 & -0.4211275 \\ \mathrm{H} & 1.0788754 & 11.2097937 & -1.8345838 \\ \mathrm{H} & -0.4885837 & 10.2461885 & -3.5190163 \\ \mathrm{H} & -0.6749349 & 7.7733539 & -3.7772832 \\ & & & \end{array}$

\section{TS $2+D$}

Imaginary Frequencies $=1(-438.72 \mathrm{~cm}-1)$

Energy (BP86/def-TZVP, level of optimization) $=-1286.5162035109 \mathrm{H}$

Energy (M0 6-2X/def2-TZVP) $=-1285.953375482 \mathrm{H}$

$\begin{array}{lrrr}\text { C } & -1.3816672 & -2.9451663 & -0.0254115 \\ \text { C } & -0.6421197 & -4.0585825 & 0.5090418 \\ \text { C } & 0.7674688 & -4.0600054 & 0.2813798 \\ \text { C } & 1.3029210 & -2.9477560 & -0.4586472 \\ \text { H } & -2.4489353 & -2.8841236 & 0.2059704 \\ \text { H } & 2.3888405 & -2.8867806 & -0.5737656\end{array}$




\begin{tabular}{lrrr}
$\mathrm{C}$ & -0.4863899 & -1.4578467 & 1.4257656 \\
$\mathrm{C}$ & 0.9047487 & -1.4630866 & 1.2106494 \\
$\mathrm{C}$ & -1.2460545 & -0.1578472 & 1.2675881 \\
$\mathrm{H}$ & -0.8820008 & -2.1101894 & 2.2040748 \\
$\mathrm{H}$ & 1.5111495 & -2.1248388 & 1.8284205 \\
$\mathrm{C}$ & 1.5945455 & -0.1685025 & 0.8376598 \\
$\mathrm{H}$ & 1.7629377 & 0.3882562 & 1.7793672 \\
$\mathrm{C}$ & -0.9275199 & -2.3686242 & -1.2591747 \\
$\mathrm{C}$ & 0.4844668 & -2.3685183 & -1.4859820 \\
$\mathrm{C}$ & 1.5641210 & -5.0709662 & 0.8689177 \\
$\mathrm{C}$ & 0.9763281 & -6.0710518 & 1.6329444 \\
$\mathrm{C}$ & -0.4189703 & -6.0691256 & 1.8591439 \\
$\mathrm{C}$ & -1.2152573 & -5.0673207 & 1.3188763 \\
$\mathrm{C}$ & -1.7843015 & -1.7697651 & -2.2160354 \\
$\mathrm{C}$ & -1.2604361 & -1.2093157 & -3.3725124 \\
$\mathrm{C}$ & 0.1371993 & -1.2069996 & -3.5961923 \\
$\mathrm{C}$ & 0.9976177 & -1.7667814 & -2.6621910 \\
$\mathrm{H}$ & 2.6442095 & -5.0642505 & 0.7039977 \\
$\mathrm{H}$ & 1.5934851 & -6.8617025 & 2.0641179 \\
$\mathrm{H}$ & -0.8704459 & -6.8579842 & 2.4639408 \\
$\mathrm{H}$ & -2.2920956 & -5.0573394 & 1.5038100 \\
$\mathrm{H}$ & -2.8629124 & -1.7702347 & -2.0414651 \\
$\mathrm{H}$ & -1.9281127 & -0.7710358 & -4.1166063 \\
$\mathrm{H}$ & 0.5377990 & -0.7666789 & -4.5112139 \\
$\mathrm{H}$ & 2.0767477 & -1.7648991 & -2.8339526 \\
$\mathrm{H}$ & -2.3259982 & -0.3303346 & 1.1640644 \\
$\mathrm{H}$ & -1.1197013 & 0.4054978 & 2.2118461 \\
$\mathrm{H}$ & 2.5922459 & -0.3500560 & 0.4152723 \\
$\mathrm{C}$ & 0.7789057 & 0.7149835 & -0.1316209 \\
$\mathrm{H}$ & 0.7812754 & 7.1709578 & -1.4850962 \\
$\mathrm{H}$ & 0.7399524 & 4.6881810 & -1.7079343 \\
$\mathrm{C}$ & 1.1994082 & 0.3962518 & -1.1608850 \\
$\mathrm{C}$ & -0.7505175 & 0.7106859 & 0.0898029 \\
$\mathrm{C}$ & -1.1309224 & 2.1694782 & 0.2779091 \\
$\mathrm{H}$ & -1.2539308 & 0.3725340 & -0.8267700 \\
$\mathrm{O}$ & 2.3209853 & 2.6344111 & -0.1285304 \\
$\mathrm{O}$ & -2.2488435 & 2.6268085 & 0.4707132 \\
$\mathrm{H}$ & 0.0384114 & 2.9490788 & 0.1922545 \\
$\mathrm{C}$ & 0.0532955 & 4.3787086 & 0.3186553 \\
$\mathrm{C}$ & -0.3303780 & 4.9685925 & 1.5282654 \\
$\mathrm{C}$ & -0.3178081 & 6.3619098 & 1.6452677 \\
$\mathrm{C}$ & 0.0834858 & 7.1557469 & 0.5643113 \\
\hline & 0.4695415 & 6.5552329 & -0.6400101 \\
$\mathrm{H}$ & -0.6306252 & 4.3430544 & 2.3693629 \\
\hline
\end{tabular}

Product $2+\mathrm{D}$

Imaginary Frequencies $=0$

Energy (BP86/def-TZVP, level of optimization) $=-1286.5782704379 \mathrm{H}$

Energy $($ M06-2X/def2-TZVP) $=-1286.031038735 \mathrm{H}$ 


\begin{tabular}{|c|c|c|c|}
\hline C & -1.2518476 & -2.5514720 & 0.3369405 \\
\hline $\mathrm{C}$ & -0.5999833 & -3.8870448 & 0.6596791 \\
\hline $\mathrm{C}$ & 0.7962914 & -3.8813757 & 0.4643350 \\
\hline $\mathrm{C}$ & 1.3230597 & -2.5420414 & -0.0267125 \\
\hline $\mathrm{H}$ & -2.3415978 & -2.5783875 & 0.4712385 \\
\hline $\mathrm{H}$ & 2.4073795 & -2.5606493 & -0.2004435 \\
\hline C & -0.5945544 & -1.5201127 & 1.3312673 \\
\hline C & 0.9592531 & -1.5091991 & 1.1065522 \\
\hline C & -1.2196347 & -0.1113563 & 1.3056411 \\
\hline $\mathrm{H}$ & -0.7946268 & -1.9184041 & 2.3369451 \\
\hline $\mathrm{H}$ & 1.4418054 & -1.8935011 & 2.0169504 \\
\hline C & 1.5304631 & -0.0930753 & 0.8929399 \\
\hline $\mathrm{H}$ & 1.5229827 & 0.4237567 & 1.8660189 \\
\hline C & -0.8531395 & -2.2124520 & -1.0861988 \\
\hline C & 0.5428364 & -2.2096522 & -1.2837591 \\
\hline C & 1.5499384 & -5.0231900 & 0.7412140 \\
\hline C & 0.9040160 & -6.1801493 & 1.2062921 \\
\hline $\mathrm{C}$ & -0.4825946 & -6.1857691 & 1.3999807 \\
\hline $\mathrm{C}$ & -1.2401423 & -5.0345321 & 1.1307044 \\
\hline C & -1.7149385 & -1.9558133 & -2.1558831 \\
\hline $\mathrm{C}$ & -1.1805303 & -1.6925171 & -3.4260852 \\
\hline C & 0.2066563 & -1.6906387 & -3.6225687 \\
\hline C & 1.0732362 & -1.9518893 & -2.5506891 \\
\hline $\mathrm{H}$ & 2.6325899 & -5.0182009 & 0.5940597 \\
\hline $\mathrm{H}$ & 1.4869332 & -7.0791147 & 1.4164146 \\
\hline $\mathrm{H}$ & -0.9784217 & -7.0891483 & 1.7607653 \\
\hline $\mathrm{H}$ & -2.3217095 & -5.0380983 & 1.2860673 \\
\hline $\mathrm{H}$ & -2.7971778 & -1.9623047 & -2.0041557 \\
\hline $\mathrm{H}$ & -1.8496180 & -1.4949930 & -4.2658650 \\
\hline $\mathrm{H}$ & 0.6156750 & -1.4917147 & -4.6150207 \\
\hline $\mathrm{H}$ & 2.1551016 & -1.9554234 & -2.7053121 \\
\hline $\mathrm{H}$ & -2.3163511 & -0.1861221 & 1.3217167 \\
\hline $\mathrm{H}$ & -0.9319148 & 0.4007842 & 2.2381129 \\
\hline $\mathrm{H}$ & 2.5843049 & -0.1554100 & 0.5854402 \\
\hline C & 0.7466274 & 0.7566727 & -0.1286944 \\
\hline $\mathrm{H}$ & 0.9796942 & 0.4095712 & -1.1454671 \\
\hline C & 1.1568706 & 2.2176621 & -0.0601134 \\
\hline C & -0.7806940 & 0.7520600 & 0.1050532 \\
\hline C & -1.1592811 & 2.2106967 & 0.3015266 \\
\hline $\mathrm{H}$ & -1.3050292 & 0.4108915 & -0.7990654 \\
\hline 0 & 2.2813298 & 2.6823122 & -0.1860363 \\
\hline O & -2.2731417 & 2.6687445 & 0.5164542 \\
\hline $\mathrm{N}$ & 0.0079746 & 2.9910569 & 0.1956591 \\
\hline C & 0.0254731 & 4.4210926 & 0.3219779 \\
\hline C & -0.2783644 & 5.0082878 & 1.5551799 \\
\hline $\mathrm{C}$ & -0.2591652 & 6.4014501 & 1.6737026 \\
\hline $\mathrm{C}$ & 0.0691181 & 7.1973746 & 0.5698381 \\
\hline $\mathrm{C}$ & 0.3744955 & 6.5995730 & -0.6586813 \\
\hline C & 0.3499981 & 5.2076164 & -0.7889886 \\
\hline $\mathrm{H}$ & -0.5220039 & 4.3805465 & 2.4129751 \\
\hline $\mathrm{H}$ & -0.4964584 & 6.8640201 & 2.6329768 \\
\hline $\mathrm{H}$ & 0.0872285 & 8.2841172 & 0.6671003 \\
\hline $\mathrm{H}$ & 0.6289281 & 7.2171220 & -1.5214286 \\
\hline
\end{tabular}




\section{TS $3+D$}

Imaginary Frequencies $=1(-418.19 \mathrm{~cm}-1)$

Energy (BP86/def-TZVP, level of optimization) $=-1401.0965193326 \mathrm{H}$

Energy (M06-2X/def2-TZVP) $=-1400.483036226 \mathrm{H}$$$
\text { c } \quad-0.9836976
$$$$
\begin{array}{ll}
-2.8202832 & 0.3212326
\end{array}
$$$$
\begin{array}{ll}
-3.8620578 & 0.5507092
\end{array}
$$$$
1.2467441
$$$$
-3.7459759
$$$$
-2.5800314
$$$$
1.4315958
$$$$
-2.2366598
$$$$
2.4065228
$$$$
0.4180903
$$$$
1.6008712
$$$$
-0.3913676
$$$$
0.3683452
$$$$
2.4433622
$$$$
2.0143239
$$$$
2.5198801
$$$$
-0.9786828
$$$$
0.2785473
$$$$
\text { 2. } 2731461
$$$$
2.0601940
$$$$
0.8221359
$$$$
-0.1882347
$$$$
-2.1340669
$$$$
-2.0448999
$$$$
-0.7984493
$$$$
0.3491314
$$$$
3.2319762
$$$$
2.8474768
$$$$
0.6561509
$$$$
-1.1428196
$$$$
-3.1056283
$$$$
-2.9466342
$$$$
-0.7428125
$$$$
\text { 1. } 3165948
$$$$
-3.1649383
$$$$
-1.4031170
$$$$
-3.9557832
$$$$
0.1022128
$$$$
2.7636752
$$$$
0.8344332
$$$$
0.6457141
$$$$
1.1623033
$$$$
-0.4817794
$$$$
-0.8716870
$$$$
-1.2772399
$$$$
\text { 2. } 1481892
$$$$
-1.8684924
$$$$
0.1237634
$$$$
0.0845662
$$$$
-2.7898806
$$$$
-2.4527062
$$$$
-1.2171415
$$$$
-1.1608399
$$$$
0.0420902
$$$$
-1.9258448
$$$$
-1.7586305
$$$$
0.1743580
$$$$
0.7364516
$$$$
-2.1934826
$$$$
-2.0815670
$$$$
-4.6850403
$$$$
-5.7426581
$$$$
-5.8663623
$$$$
-4.9360327
$$$$
-1.6586095
$$$$
-1.0570748
$$$$
-0.9482250
$$$$
-1.4443073
$$$$
-4.5751472
$$$$
-6.4791549
$$$$
-6.7024113
$$$$
-5.0503874
$$$$
-1.7511449
$$$$
-0.6741862
$$$$
-0.4791305
$$$$
-1.3632750
$$$$
-3.8024739
$$$$
-0.1696908
$$$$
-0.1268140
$$$$
-0.9540065
$$$$
1.1567138
$$$$
-1.4334626
$$$$
1.4307836
$$$$
0.6690552
$$$$
\text { 1. } 6203348
$$$$
\text { 2. } 2568847
$$$$
1.0185330
$$$$
0.0790547
$$$$
0.8854546
$$$$
-0.9802517
$$$$
-1.6517029
$$$$
0.1231779
$$$$
0.9974049
$$$$
\text { 1. } 6650368
$$$$
\text { 1. } 4574388
$$$$
-1.6088206
$$$$
-2.8563575
$$$$
-3.5156192
$$$$
-2.9141210
$$$$
-0.3894067
$$$$
1.1690273
$$$$
\text { 2. } 3473871
$$$$
\text { 1. } 9723489
$$$$
-1.1216252
$$$$
-3.3382696
$$$$
-4.4995476
$$$$
-3.4153011
$$$$
0.6899887
$$$$
\text { 1. } 9924320
$$$$
-3.7444356 \quad 1.2580321
$$$$
0.6278423 \quad 2.4207576
$$$$
0.0460878-0.7139257
$$$$
1.0143941-0.4578562
$$$$
0.7258974-1.5021322
$$$$
2.4977883-0.4828397
$$$$
0.9048112 \quad 0.3435006
$$$$
2.3358893 \quad 0.6707373
$$$$
0.4968794-0.2961958
$$$$
3.0330438 \quad-0.9694601
$$$$
2.7145253 \quad 1.2697354
$$$$
3.1940902 \quad 0.1665054
$$$$
4.6228102 \quad 0.2981393
$$

5.2001477

1.5722805 


$\begin{array}{rrrr}\text { C } & 0.0779190 & 6.5921916 & 1.6948975 \\ \text { C } & 0.0051571 & 7.3980316 & 0.5524513 \\ \text { C } & -0.0277440 & 6.8105228 & -0.7177145 \\ \text { C } & 0.0069428 & 5.4191121 & -0.8503032 \\ \text { H } & 0.1927428 & 4.5666300 & 2.4570929 \\ \text { H } & 0.1055010 & 7.0468334 & 2.6864429 \\ \text { H } & -0.0261751 & 8.4842493 & 0.6522391 \\ \text { H } & -0.0861778 & 7.4351936 & -1.6103004 \\ \text { H } & -0.0303887 & 4.9530750 & -1.8354436 \\ \text { H } & -2.7080147 & -1.7955505 & 1.0909835 \\ \text { H } & -1.9845481 & -2.9698804 & 2.2156319\end{array}$

\section{Product $3+D$}

Imaginary Frequencies $=0$

Energy(BP86/def-TZVP, level of optimization) $=-1401.1556385629 \mathrm{H}$ Energy (M06-2X/def2-TZVP) $=-1400.562311703 \mathrm{H}$

$\begin{array}{lrrr}\text { C } & 1.1788009 & 0.3811423 & -2.0556819 \\ \mathrm{C} & 2.0085387 & -0.9122051 & -2.0294411 \\ \mathrm{C} & 3.2421628 & -0.8046000 & -2.7031829 \\ \mathrm{C} & 3.4820597 & 0.5657069 & -3.3074306 \\ \mathrm{C} & -0.1843590 & 0.2530534 & -1.3781819 \\ \mathrm{H} & 4.4340610 & 0.6172756 & -3.8530398 \\ \mathrm{C} & 2.1064153 & 1.4610873 & -1.3443332 \\ \mathrm{C} & 3.4794397 & 1.5664026 & -2.0970245 \\ \mathrm{C} & 1.4607263 & 2.8418998 & -1.1116118 \\ \mathrm{H} & 2.2929933 & 1.0480312 & -0.3442810 \\ \mathrm{H} & 4.2700284 & 1.2111516 & -1.4202066 \\ \mathrm{C} & 3.8636615 & 3.0134554 & -2.4687962 \\ \mathrm{H} & 4.1736558 & 3.5296804 & -1.5460589 \\ \mathrm{C} & 1.0526056 & 0.7440813 & -3.5380714 \\ \mathrm{C} & 2.2933600 & 0.8451288 & -4.2009615 \\ \mathrm{C} & 4.1159914 & -1.8915492 & -2.7662914 \\ \mathrm{C} & 3.7548715 & -3.1047518 & -2.1618523 \\ \mathrm{C} & 2.5297021 & -3.2171159 & -1.4953881 \\ \mathrm{C} & 1.6552161 & -2.1214411 & -1.4234827 \\ \mathrm{C} & -0.1326372 & 0.9372054 & -4.2579669 \\ \mathrm{C} & -0.0764442 & 1.2331812 & -5.6284197 \\ \mathrm{C} & 1.1571355 & 1.3334926 & -6.2815584 \\ \mathrm{C} & 2.3467003 & 1.1365876 & -5.5662698 \\ \mathrm{H} & 5.0705779 & -1.7980058 & -3.2900345 \\ \mathrm{H} & 4.4300829 & -3.9611650 & -2.2136633 \\ \mathrm{H} & 2.2493672 & -4.1623154 & -1.0262194 \\ \mathrm{H} & 0.7124585 & -2.2142937 & -0.8834798 \\ \mathrm{H} & -1.1054909 & 0.8612604 & -3.7697715 \\ \mathrm{H} & -1.0032053 & 1.3793925 & -6.1864801 \\ \mathrm{H} & 1.1941406 & 1.5579668 & -7.3492048 \\ \mathrm{H} & 3.3127551 & 1.2067729 & -6.0723294 \\ \mathrm{O} & -0.0109487 & 0.0590405 & 0.0358547 \\ \mathrm{H} & 0.4599583 & 2.7351165 & -0.6723416 \\ \mathrm{H} & -0.9001069 & -0.0304208 & 0.4231095 \\ \mathrm{H} & 2.0672630 & 3.3638721 & -0.3540504 \\ \mathrm{H} & 4.7436999 & 3.0086224 & -3.1280112 \\ \mathrm{C} & 2.7263968 & 3.8164344 & -3.1333700\end{array}$




$\begin{array}{rrrr}\mathrm{H} & 2.6066316 & 3.4853389 & -4.1748291 \\ \mathrm{C} & 3.0480572 & 5.3004006 & -3.1829541 \\ \mathrm{C} & 1.3828895 & 3.7170864 & -2.3778179 \\ \mathrm{C} & 1.0055432 & 5.1497216 & -2.0417706 \\ \mathrm{H} & 0.5948017 & 3.3314743 & -3.0410270 \\ \mathrm{O} & 4.0312781 & 5.8327812 & -3.6791360 \\ \mathrm{O} & 0.0094716 & 5.5367324 & -1.4465572 \\ \mathrm{~N} & 2.0122343 & 6.0021543 & -2.5356357 \\ \mathrm{C} & 1.9799561 & 7.4313348 & -2.4046216 \\ \mathrm{C} & 2.0475393 & 8.0091644 & -1.1319815 \\ \mathrm{C} & 2.0100968 & 9.4014711 & -1.0090872 \\ \mathrm{C} & 1.9155380 & 10.2069167 & -2.1502443 \\ \mathrm{C} & 1.8532124 & 9.6189172 & -3.4190889 \\ \mathrm{C} & 1.8807078 & 8.2272607 & -3.5515691 \\ \mathrm{H} & 2.1335357 & 7.3755612 & -0.2485854 \\ \mathrm{H} & 2.0606116 & 9.8565941 & -0.0186598 \\ \mathrm{H} & 1.8902251 & 11.2933044 & -2.0504544 \\ \mathrm{H} & 1.7778396 & 10.2434513 & -4.3105217 \\ \mathrm{H} & 1.8213274 & 7.7605103 & -4.5353038 \\ \mathrm{H} & -0.7346104 & -0.5966237 & -1.8209928 \\ \mathrm{H} & -0.7791292 & 1.1632942 & -1.5648913 \\ & & & \end{array}$

TS $4+D$ (endo)

Imaginary Frequencies $=1(-419.72 \mathrm{~cm}-1)$

Energy (BP86/def-TZVP, level of optimization) $=-1130.8667427925 \mathrm{H}$

Energy (M0 6-2X/def2-TZVP) $=-1130.346836348 \mathrm{H}$

$\begin{array}{lrrr}\mathrm{C} & 0.7132090 & -0.2494683 & 3.7962449 \\ \mathrm{C} & 0.0700260 & -1.3955360 & 3.3216351 \\ \mathrm{C} & -1.3172297 & -1.4588223 & 3.1856207 \\ \mathrm{C} & -2.1427596 & -0.3591218 & 3.4815990 \\ \mathrm{H} & 0.6840662 & -2.1947065 & 2.8973463 \\ \mathrm{H} & -1.7538540 & -2.3196239 & 2.6703977 \\ \mathrm{C} & -0.0929340 & 1.4529298 & 2.3126450 \\ \mathrm{C} & -1.4673449 & 1.2133326 & 2.1625815 \\ \mathrm{C} & 0.8072869 & 1.2988535 & 1.1123790 \\ \mathrm{C} & -1.9549397 & 0.8374577 & 0.7767872 \\ \mathrm{C} & -1.0469390 & -0.2171701 & 0.1081804 \\ \mathrm{C} & 0.4653553 & 0.0418920 & 0.2829117 \\ \mathrm{C} & 2.1683616 & -0.1293725 & 3.7366713 \\ \mathrm{H} & 0.2175915 & 0.4038386 & 4.5113951 \\ \mathrm{H} & -1.8231591 & 0.2921083 & 4.2983524 \\ \mathrm{C} & -3.6338237 & -0.4387998 & 3.2992393 \\ \mathrm{H} & -4.0856766 & 0.5547834 & 3.1657200 \\ \mathrm{H} & -3.9105190 & -1.0702824 & 2.4430396 \\ \mathrm{H} & -4.0937773 & -0.8788347 & 4.2000251 \\ \mathrm{O} & 2.9365591 & -0.8415575 & 3.0866952 \\ \mathrm{O} & 2.6267461 & 0.9268282 & 4.4784293 \\ \mathrm{H} & 3.6005026 & 0.9647807 & 4.3646555 \\ \mathrm{H} & 0.2382250 & 2.1537408 & 3.0791593 \\ \mathrm{H} & -2.1404589 & 1.8571424 & 2.7323649 \\ \mathrm{H} & 1.8700423 & 1.2845498 & 1.3871653 \\ \mathrm{H} & 0.6695462 & 2.1977742 & 0.4801476 \\ \mathrm{H} & -1.9604740 & 1.7560331 & 0.1609670\end{array}$




$\begin{array}{rrrr}\mathrm{H} & -2.9911830 & 0.4752894 & 0.7927214 \\ \mathrm{C} & -1.2709244 & -0.2838590 & -1.3933497 \\ \mathrm{H} & -1.3096667 & -1.2057873 & 0.5122018 \\ \mathrm{H} & 0.9454817 & -0.8250492 & 0.7609223 \\ \mathrm{C} & 1.0223024 & 0.1284312 & -1.1290123 \\ \mathrm{O} & -2.3260917 & -0.4813969 & -1.9785162 \\ \mathrm{O} & 2.1785193 & 0.3475881 & -1.4593205 \\ \mathrm{~N} & -0.0355574 & -0.0754147 & -2.0363907 \\ \mathrm{C} & 0.1289099 & -0.0793436 & -3.4626774 \\ \mathrm{C} & -0.4987440 & 0.9065039 & -4.2323087 \\ \mathrm{C} & -0.3406511 & 0.8945805 & -5.6215671 \\ \mathrm{C} & 0.4457618 & -0.0893934 & -6.2323552 \\ \mathrm{C} & 1.0730951 & -1.0683081 & -5.4525494 \\ \mathrm{C} & 0.9136863 & -1.0701516 & -4.0634178 \\ \mathrm{H} & -1.1000028 & 1.6766294 & -3.7481105 \\ \mathrm{H} & -0.8297710 & 1.6600906 & -6.2259080 \\ \mathrm{H} & 0.5699709 & -0.0933030 & -7.3164765 \\ \mathrm{H} & 1.6852800 & -1.8377744 & -5.9254448 \\ \mathrm{H} & 1.3892914 & -1.8360309 & -3.4498337\end{array}$

\section{TS $4+D$ (exo)}

Imaginary Frequencies $=1(-428.71 \mathrm{~cm}-1)$

Energy (BP86/def-TZVP, level of optimization) $=-1130.8652746823 \mathrm{H}$

Energy (M0 6-2X/def2-TZVP) $=-1130.343365319 \mathrm{H}$

\begin{tabular}{|c|c|c|c|}
\hline C & -0.7162261 & 0.5783711 & -3.6191513 \\
\hline C & 0.1190245 & 0.2899406 & -4.7026057 \\
\hline $\mathrm{C}$ & 1.4990833 & 0.1735587 & -4.5674698 \\
\hline $\mathrm{C}$ & 2.1604888 & 0.3187144 & -3.3342284 \\
\hline $\mathrm{H}$ & -0.3496767 & -0.0166043 & -5.6415981 \\
\hline $\mathrm{H}$ & 2.0692499 & -0.2232281 & -5.4129716 \\
\hline $\mathrm{C}$ & -0.1251809 & -1.2665671 & -2.2280971 \\
\hline $\mathrm{C}$ & 1.2720434 & -1.2162432 & -2.0801484 \\
\hline $\mathrm{H}$ & -0.5484472 & -1.8802014 & -3.0220540 \\
\hline $\mathrm{C}$ & -0.9915929 & -1.0646970 & -1.0097040 \\
\hline $\mathrm{H}$ & 1.8597719 & -1.9337297 & -2.6538318 \\
\hline $\mathrm{C}$ & 1.7921199 & -0.9233304 & -0.6852551 \\
\hline $\mathrm{C}$ & 1.0209344 & 0.2273231 & -0.0031875 \\
\hline $\mathrm{C}$ & -0.5136592 & 0.1166791 & -0.1384053 \\
\hline C & -2.1689228 & 0.5181640 & -3.7718832 \\
\hline $\mathrm{H}$ & -0.3549534 & 1.1823355 & -2.7883611 \\
\hline $\mathrm{H}$ & 1.7539973 & 1.0487243 & -2.6289801 \\
\hline C & 3.6509651 & 0.1232823 & -3.2606071 \\
\hline $\mathrm{H}$ & 3.9883845 & -0.1813042 & -2.2603875 \\
\hline $\mathrm{H}$ & 3.9943653 & -0.6259774 & -3.9876055 \\
\hline $\mathrm{H}$ & 4.1628681 & 1.0742165 & -3.4849617 \\
\hline O & -2.7876010 & -0.0261379 & -4.6889997 \\
\hline O & -2.8206722 & 1.1322917 & -2.7355571 \\
\hline $\mathrm{H}$ & -3.7838593 & 1.0239511 & -2.8885447 \\
\hline $\mathrm{H}$ & -0.9446295 & -1.9979679 & -0.4152972 \\
\hline $\mathrm{H}$ & -2.0470600 & -0.9236286 & -1.2731661 \\
\hline $\mathrm{H}$ & 2.8658048 & -0.6947396 & -0.6839351 \\
\hline $\mathrm{H}$ & 1.6801953 & -1.8435750 & -0.0824925 \\
\hline H & 1.3777594 & 1.1866676 & -0.4067420 \\
\hline
\end{tabular}




$\begin{array}{rrrr}\mathrm{C} & 1.2891431 & 0.2565503 & 1.4938499 \\ \mathrm{C} & -1.0351218 & 0.0221619 & 1.2875383 \\ \mathrm{H} & -0.9289883 & 1.0462049 & -0.5560230 \\ \mathrm{O} & -2.1957777 & -0.1111844 & 1.6457173 \\ \mathrm{O} & 2.3721381 & 0.3596887 & 2.0507094 \\ \mathrm{~N} & 0.0605864 & 0.1195030 & 2.1674131 \\ \mathrm{C} & -0.0643838 & 0.0919655 & 3.5976984 \\ \mathrm{C} & 0.2931738 & 1.2210594 & 4.3431321 \\ \mathrm{C} & 0.1720062 & 1.1876865 & 5.7357753 \\ \mathrm{C} & -0.3106364 & 0.0388308 & 6.3738802 \\ \mathrm{C} & -0.6693190 & -1.0835002 & 5.6181180 \\ \mathrm{C} & -0.5433313 & -1.0633122 & 4.2256447 \\ \mathrm{H} & 0.6570277 & 2.1161928 & 3.8376687 \\ \mathrm{H} & 0.4509366 & 2.0647561 & 6.3217428 \\ \mathrm{H} & -0.4070188 & 0.0181747 & 7.4605771 \\ \mathrm{H} & -1.0442916 & -1.9813323 & 6.1118285 \\ \mathrm{H} & -0.8101308 & -1.9375431 & 3.6310696\end{array}$

\section{Product $4+\mathrm{D}$ (endo)}

Imaginary Frequencies $=0$

Energy (BP86/def-TZVP, level of optimization) $=-1130.9284213086 \mathrm{H}$

Energy (M06-2X/def2-TZVP) $=-1130.431323492 \mathrm{H}$

$\begin{array}{lrrr}\text { C } & -1.5090206 & -0.0295453 & -0.0655018 \\ \mathrm{C} & -2.0414851 & -1.4141765 & -0.3078362 \\ \mathrm{C} & -3.3683917 & -1.5403231 & -0.4583279 \\ \mathrm{C} & -4.2246435 & -0.3001686 & -0.3883234 \\ \mathrm{H} & -1.3523446 & -2.2594182 & -0.3508774 \\ \mathrm{H} & -3.8356686 & -2.5145511 & -0.6276841 \\ \mathrm{C} & -2.0780118 & 1.0099374 & -1.1105953 \\ \mathrm{C} & -3.6344576 & 0.8408155 & -1.2912210 \\ \mathrm{C} & -1.3293111 & 0.9750239 & -2.4611837 \\ \mathrm{C} & -4.0145034 & 0.7091621 & -2.7821754 \\ \mathrm{C} & -3.2255905 & -0.4034209 & -3.4973148 \\ \mathrm{C} & -1.7000709 & -0.2515505 & -3.3155520 \\ \mathrm{C} & 0.0027339 & 0.0346015 & 0.0072150 \\ \mathrm{H} & -1.8747810 & 0.3264899 & 0.9154859 \\ \mathrm{H} & -4.1353308 & 0.0926053 & 0.6438317 \\ \mathrm{C} & -5.7131277 & -0.5750331 & -0.6297381 \\ \mathrm{H} & -6.2995493 & 0.3552496 & -0.6060193 \\ \mathrm{H} & -5.8902723 & -1.0646181 & -1.5989875 \\ \mathrm{H} & -6.1064706 & -1.2415845 & 0.1517862 \\ \mathrm{O} & 0.7780906 & -0.8313995 & -0.3725974 \\ \mathrm{O} & 0.4267829 & 1.2045793 & 0.5504973 \\ \mathrm{H} & 1.4089402 & 1.2018502 & 0.5490562 \\ \mathrm{H} & -1.8900679 & 2.0024127 & -0.6779172 \\ \mathrm{H} & -4.1071801 & 1.7706441 & -0.9395081 \\ \mathrm{H} & -0.2410649 & 1.0041690 & -2.3097044 \\ \mathrm{H} & -1.5792138 & 1.8925539 & -3.0158128 \\ \mathrm{H} & -3.8165763 & 1.6712427 & -3.2806859 \\ \mathrm{H} & -5.0918980 & 0.5295632 & -2.8912711 \\ \mathrm{C} & -3.4534435 & -0.3883652 & -4.9992960 \\ \mathrm{H} & -3.5657697 & -1.3842608 & -3.1313599 \\ \mathrm{H} & -1.2691016 & -1.1563289 & -2.8602233\end{array}$




$\begin{array}{lrrr}\mathrm{C} & -1.1363039 & -0.1497807 & -4.7231126 \\ \mathrm{O} & -4.5185524 & -0.4856871 & -5.5924121 \\ \mathrm{O} & 0.0333545 & -0.0029190 & -5.0480326 \\ \mathrm{~N} & -2.2056338 & -0.2370596 & -5.6360881 \\ \mathrm{C} & -2.0393154 & -0.1916964 & -7.0615664 \\ \mathrm{C} & -2.5660830 & 0.8845238 & -7.7842928 \\ \mathrm{C} & -2.4038887 & 0.9220577 & -9.1726933 \\ \mathrm{C} & -1.7140511 & -0.1040216 & -9.8290320 \\ \mathrm{C} & -1.1876824 & -1.1743130 & -9.0960840 \\ \mathrm{C} & -1.3518279 & -1.2248119 & -7.7083842 \\ \mathrm{H} & -3.0926777 & 1.6855475 & -7.2643306 \\ \mathrm{H} & -2.8140302 & 1.7586017 & -9.7405597 \\ \mathrm{H} & -1.5861786 & -0.0695238 & -10.9121698 \\ \mathrm{H} & -0.6510752 & -1.9768167 & -9.6045909 \\ \mathrm{H} & -0.9546091 & -2.0603829 & -7.1309518\end{array}$

\section{Product $4+\mathrm{D}$ (exo)}

Imaginary Frequencies $=0$

Energy (BP86/def-TZVP, level of optimization) $=-1130.9322410011 \mathrm{H}$

Energy (M06-2X/def2-TZVP) $=-1130.432717956 \mathrm{H}$

$\begin{array}{lrrr}\mathrm{C} & 1.4997557 & 0.1387240 & 0.3217253 \\ \mathrm{C} & 2.3265252 & 0.1921234 & -0.9413307 \\ \mathrm{C} & 3.6510473 & 0.0218505 & -0.8496647 \\ \mathrm{C} & 4.3146929 & -0.2067421 & 0.4850205 \\ \mathrm{H} & 1.8289930 & 0.3629433 & -1.8980451 \\ \mathrm{H} & 4.2798365 & 0.0655434 & -1.7437501 \\ \mathrm{C} & 1.9273020 & -1.1154563 & 1.1613417 \\ \mathrm{C} & 3.4798349 & -1.1662677 & 1.3956107 \\ \mathrm{H} & 1.6514472 & -1.9824200 & 0.5437421 \\ \mathrm{C} & 1.1540527 & -1.2300498 & 2.4894066 \\ \mathrm{H} & 3.7966504 & -2.1863010 & 1.1274887 \\ \mathrm{C} & 3.8448895 & -0.9694203 & 2.8871092 \\ \mathrm{C} & 3.0365162 & 0.1694069 & 3.5422271 \\ \mathrm{C} & 1.5147531 & -0.0760852 & 3.4416712 \\ \mathrm{C} & 0.0184689 & 0.0668746 & 0.0083833 \\ \mathrm{H} & 1.6772904 & 1.0484927 & 0.9187111 \\ \mathrm{H} & 4.3580553 & 0.7747488 & 0.9979817 \\ \mathrm{C} & 5.7569064 & -0.7088508 & 0.3146385 \\ \mathrm{H} & 6.2569264 & -0.8565729 & 1.2822545 \\ \mathrm{H} & 5.7694791 & -1.6687088 & -0.2247326 \\ \mathrm{H} & 6.3539623 & 0.0132566 & -0.2614781 \\ \mathrm{O} & -0.4909950 & -0.7189218 & -0.7785226 \\ \mathrm{O} & -0.7059809 & 0.9709120 & 0.7136702 \\ \mathrm{H} & -1.6535029 & 0.8512489 & 0.4810720 \\ \mathrm{H} & 1.4001587 & -2.1956460 & 2.9569234 \\ \mathrm{H} & 0.0691086 & -1.2436584 & 2.3152637 \\ \mathrm{H} & 4.9183400 & -0.7599156 & 2.9914568 \\ \mathrm{H} & 3.6585611 & -1.9038480 & 3.4391540 \\ \mathrm{H} & 3.3262834 & 1.1331552 & 3.0974173 \\ \mathrm{C} & 3.3259324 & 0.2486438 & 5.0321954 \\ \mathrm{C} & 1.0544591 & -0.3134182 & 4.8706139 \\ \mathrm{H} & 1.0010643 & 0.8415599 & 3.1128380 \\ \mathrm{O} & -0.0610733 & -0.6392629 & 5.2498878\end{array}$




$\begin{array}{lrrr}\mathrm{O} & 4.3914685 & 0.5118479 & 5.5708635 \\ \mathrm{~N} & 2.1455669 & -0.0754867 & 5.7291344 \\ \mathrm{C} & 2.0601628 & -0.1452426 & 7.1610500 \\ \mathrm{C} & 2.2167925 & 1.0212902 & 7.9182031 \\ \mathrm{C} & 2.1361033 & 0.9480347 & 9.3122858 \\ \mathrm{C} & 1.8924128 & -0.2793554 & 9.9399538 \\ \mathrm{C} & 1.7324552 & -1.4393144 & 9.1726103 \\ \mathrm{C} & 1.8200365 & -1.3777587 & 7.7783508 \\ \mathrm{H} & 2.3947639 & 1.9749104 & 7.4198552 \\ \mathrm{H} & 2.2593231 & 1.8541182 & 9.9074764 \\ \mathrm{H} & 1.8270416 & -0.3320241 & 11.0279030 \\ \mathrm{H} & 1.5432674 & -2.3975579 & 9.6588590 \\ \mathrm{H} & 1.7067679 & -2.2783469 & 7.1738280\end{array}$

TS $5+\mathrm{D}$ (endo)

Imaginary Frequencies $=1(-455.02 \mathrm{~cm}-1)$

Energy(BP86/def-TZVP, level of optimization) $=-1471.8126661809 \mathrm{H}$

Energy (M06-2X/def2-TZVP) $=-1471.131629264 \mathrm{H}$

$-1.0898767$

2.3652519

0.5522056

C $\quad-0.7107611$

1.8946827

$\begin{array}{ll}\text { C } & 0.6702266 \\ \text { C } & 1.1028473\end{array}$

1.8938135

$-0.7385899$

2. 3640249

$-0.7670130$

0.0187297

3.0102496

$-1.3947574$

1.5124875

1. 3221837

1.5109387

0.5068929

1.0997946

$-1.4919962$

$-2.4265662$

2.8993311

$-1.5479505$

0.9823972

$-2.4294135$

3.0576157

$-3.2047237$

2.1648795

2.0703537

0.7380481

2. 4566457

2.8957191

0.8819531

2. 5057992

3.0506490

1.9694719

3.2234008

2.1616655

0.6031105

$-2.7980389$

4.1494715

0.3186746

2.7998417

4.1472937

0.2056863

$-3.7709546$

4.2368611

$-0.6283377$

3.7547895

4.2444017

$-0.7584021$

3. 9555084

5.6258919

$-1.3472398$

3. 7893044

5.5759919

$-2.4324485$

3.2876654

6.3832669

$-0.9175255$

4.9980218

5.9333377

$-1.1850941$

4.4300300

3.2679371

$-1.1347508$

2.2820979

4.9839452

0.4618139

$-3.9952944$

5.6158319

$-1.2145711$

6.3844582

$-0.7805971$

$-3.3432818$

5.5715131

$-2.2991979$

-3.8228391
-5.0440614

5.9031600

$-1.0568861$

$-4.4421254$

3.2531848

$-0.9924458$

4.9903844

0.5639653

0.7364622

0.6384259

1.7544441

$-0.6767051$

0.6400959

1.7834253

1.2525592

1.1514470

2.5693469

1.4517053

$-0.6037861$

1. 2638646

1.1546256

2. 6187437

$-1.1575551$

$-0.6007986$

1. 3246728 


$\begin{array}{lrrr}\mathrm{H} & -2.4747592 & -0.3912111 & 1.1215021 \\ \mathrm{H} & -1.4071646 & -1.3249311 & 2.1612342 \\ \mathrm{H} & 1.4745496 & -1.3305255 & 2.0980023 \\ \mathrm{H} & 2.5031276 & -0.3974717 & 1.0182137 \\ \mathrm{C} & -0.7799845 & -1.2629474 & 0.0818538 \\ \mathrm{H} & -1.1697903 & -0.7614963 & -0.8159999 \\ \mathrm{C} & -1.1774841 & -2.7251729 & -0.0375125 \\ \mathrm{C} & 0.7646052 & -1.2579167 & 0.0446512 \\ \mathrm{C} & 1.1652359 & -2.7158926 & -0.1080283 \\ \mathrm{H} & 1.1105645 & -0.7444996 & -0.8638675 \\ \mathrm{O} & 2.2976804 & -3.1710632 & -0.1885925 \\ \mathrm{O} & -2.3081972 & -3.1910375 & -0.0334860 \\ \mathrm{~N} & -0.0050090 & -3.4978528 & -0.1457385 \\ \mathrm{C} & -0.0030452 & -4.9261078 & -0.2893813 \\ \mathrm{C} & -0.5561127 & -5.5051329 & -1.4371913 \\ \mathrm{C} & -0.5551550 & -6.8970647 & -1.5703779 \\ \mathrm{C} & 0.0021186 & -7.7000810 & -0.5682186 \\ \mathrm{C} & 0.5573032 & -7.1104194 & 0.5736608 \\ \mathrm{C} & 0.5533363 & -5.7198493 & 0.7199123 \\ \mathrm{H} & -0.9780073 & -4.8715900 & -2.2180603 \\ \mathrm{H} & -0.9869446 & -7.3530572 & -2.4626465 \\ \mathrm{H} & 0.0038048 & -8.7858614 & -0.6769583 \\ \mathrm{H} & 0.9912432 & -7.7333226 & 1.3573551 \\ \mathrm{H} & 0.9736477 & -5.2535064 & 1.6115350\end{array}$

TS $5+\mathrm{D}$ (exo)

Imaginary Frequencies $=1(-439.12 \mathrm{~cm}-1)$

Energy (BP86/def-TZVP, level of optimization) $=-1471.8133126848 \mathrm{H}$

Energy (M06-2X/def2-TZVP) $=-1471.136541172 \mathrm{H}$

C $\quad 0.4595783 \quad 2.5700060 \quad 1.4077112$

C $\quad-0.9081317 \quad 2.4656994 \quad 1.2749951$

C $\quad-1.1652052 \quad 2.2634667 \quad-0.1128086$

$\begin{array}{llll}0 & -0.0243807 & 2.6220732 & -0.8138637\end{array}$

$\mathrm{H} \quad 1.0391214 \quad 2.6056089 \quad 2.3267315$

$\mathrm{H} \quad-1.6508367 \quad 2.3976546 \quad 2.0654875$

C $\quad 2.3762336 \quad 2.8466235 \quad-0.3614200$

$\mathrm{H} \quad 2.5807843 \quad 2.4546098 \quad-1.3664485$

$\begin{array}{llll}\mathrm{H} & 3.1277672 & 2.4427557 & 0.3289725\end{array}$

C $\quad \begin{array}{llll}-2.4598701 & 2.4911464 & -0.8500138\end{array}$

$\mathrm{H} \quad-2.6378004 \quad 3.5712259 \quad-0.9641710$

$\mathrm{H} \quad-2.4076725 \quad 2.0536688 \quad-1.8577037$

$\begin{array}{llll}\mathrm{N} & 2.5501844 & 4.3001501 & -0.3887427\end{array}$

$\mathrm{N} \quad-3.5998111 \quad 1.9481324 \quad-0.1291326$

$\begin{array}{lrrr}\mathrm{N} & -3.5998111 & 1.9481324 & -0.1291326\end{array}$

C $\quad-4.5495704 \quad 2.7222625 \quad 0.4680152$

C $\quad-5.6745989 \quad 1.9700518 \quad 1.1476115$

$\mathrm{H} \quad-6.6282499 \quad 2.2717446 \quad 0.6924235$

$\mathrm{H} \quad-5.5730225 \quad 0.8797864 \quad 1.0784835$

$\mathrm{H} \quad-5.7065822 \quad 2.2610391 \quad 2.2067041$

$\begin{array}{llll}0 & -4.5032696 & 3.9652104 & 0.4570513\end{array}$

$\mathrm{H} \quad-3.7131779 \quad 0.9385726 \quad-0.0955751$

$\begin{array}{llll}\text { C } & 3.2923501 & 6.4933321 & 0.3683411\end{array}$ 


$\begin{array}{rrrr}\mathrm{H} & 2.7655261 & 6.8369357 & -0.5308849 \\ \mathrm{H} & 2.8460701 & 6.9746110 & 1.2500081 \\ \mathrm{H} & 4.3427943 & 6.8109068 & 0.3128966 \\ \mathrm{O} & 3.8117388 & 4.4310344 & 1.5125644 \\ \mathrm{H} & 2.0982918 & 4.8196065 & -1.1365854 \\ \mathrm{C} & 0.8230552 & 0.2794994 & -0.0497332 \\ \mathrm{C} & -0.5848691 & 0.1479342 & -0.1789986 \\ \mathrm{H} & 1.2767356 & 0.0434953 & 0.9138051 \\ \mathrm{C} & 1.6394108 & -0.0943373 & -1.2721552 \\ \mathrm{H} & -1.1560439 & -0.1743904 & 0.6923745 \\ \mathrm{C} & -1.0884591 & -0.3283581 & -1.5254493 \\ \mathrm{H} & 1.4863774 & 0.6315091 & -2.0865813 \\ \mathrm{H} & 2.7155175 & -0.1160599 & -1.0543960 \\ \mathrm{H} & -0.9193272 & 0.4343435 & -2.3028046 \\ \mathrm{H} & -2.1658866 & -0.5439931 & -1.5137787 \\ \mathrm{C} & -0.3268614 & -1.6044256 & -1.9850954 \\ \mathrm{H} & -0.6089750 & -1.8310619 & -3.0243129 \\ \mathrm{C} & 1.2016745 & -1.4772359 & -1.8297802 \\ \mathrm{C} & 1.6052582 & -2.6153193 & -0.9032564 \\ \mathrm{H} & 1.7177518 & -1.6523048 & -2.7861807 \\ \mathrm{C} & -0.7146797 & -2.8144270 & -1.1484326 \\ \mathrm{O} & -1.8362009 & -3.2731730 & -0.9825710 \\ \mathrm{O} & 2.7300640 & -2.8877022 & -0.5080511 \\ \mathrm{~N} & 0.4500433 & -3.3441988 & -0.5624150 \\ \mathrm{C} & 0.4579604 & -4.5049608 & 0.2821015 \\ \mathrm{C} & 0.8264045 & -4.3797867 & 1.6264593 \\ \mathrm{C} & 0.8341837 & -5.5147363 & 2.4433534 \\ \mathrm{C} & 0.4673757 & -6.7608261 & 1.9212223 \\ \mathrm{C} & 0.0962011 & -6.8745184 & 0.5760524 \\ \mathrm{C} & 0.0937260 & -5.7468946 & -0.2507921 \\ \mathrm{H} & 1.0998495 & -3.4042005 & 2.0293755 \\ \mathrm{H} & 1.1224150 & -5.4219379 & 3.4915441 \\ \mathrm{H} & 0.4706674 & -7.6436682 & 2.5624699 \\ \mathrm{H} & -0.1885801 & -7.8443839 & 0.1652998 \\ \mathrm{H} & -0.1841393 & -5.8284147 & -1.3021018 \\ & & & \end{array}$

\section{Product $5+\mathrm{D}$ (endo)}

Imaginary Frequencies $=0$

Energy (BP86/def-TZVP, level of optimization) $=-1471.8553164001 \mathrm{H}$ Energy (M06-2X/def2-TZVP) $=-1471.190473411 \mathrm{H}$

$\begin{array}{lrrr}\text { C } & -1.0800411 & 2.1214392 & 0.7879666 \\ \mathrm{C} & -0.6853439 & 1.8705939 & -0.6626504 \\ \mathrm{C} & 0.6556282 & 1.8703384 & -0.6864373 \\ \mathrm{C} & 1.1016829 & 2.1207948 & 0.7494215 \\ \mathrm{O} & 0.0192397 & 2.9825213 & 1.2315452 \\ \mathrm{H} & -1.3788350 & 1.6625618 & -1.4754858 \\ \mathrm{H} & 1.3197952 & 1.6620222 & -1.5233338 \\ \mathrm{C} & -2.4251703 & 2.7644795 & 1.0689636 \\ \mathrm{H} & -2.5103263 & 2.9656838 & 2.1487693 \\ \mathrm{H} & -3.2313660 & 2.0745562 & 0.7863654 \\ \mathrm{C} & 2.4558625 & 2.7636826 & 0.9828162 \\ \mathrm{H} & 2.5780356 & 2.9667441 & 2.0588394 \\ \mathrm{H} & 3.2521825 & 2.0733252 & 0.6743406\end{array}$




\begin{tabular}{|c|c|c|c|}
\hline $\mathrm{N}$ & -2.6283319 & 3.9957053 & 0.3189522 \\
\hline $\mathrm{N}$ & 2.6326717 & 3.9931385 & 0.2232551 \\
\hline C & -3.6194673 & 4.1720134 & -0.5954991 \\
\hline C & 3.6038673 & 4.1740970 & -0.7114561 \\
\hline C & 3.6120550 & 5.5251573 & -1.3975695 \\
\hline $\mathrm{H}$ & 3.4856967 & 5.3747817 & -2.4788223 \\
\hline $\mathrm{H}$ & 2.8265717 & 6.2011670 & -1.0362746 \\
\hline $\mathrm{H}$ & 4.5919139 & 5.9974462 & -1.2408988 \\
\hline O & 4.4403122 & 3.2939334 & -0.9907402 \\
\hline $\mathrm{H}$ & 1.9796887 & 4.7525878 & 0.3986203 \\
\hline C & -3.6511556 & 5.5247487 & -1.2775637 \\
\hline $\mathrm{H}$ & -2.8708533 & 6.2095747 & -0.9217211 \\
\hline $\mathrm{H}$ & -3.5325319 & 5.3788746 & -2.3603909 \\
\hline $\mathrm{H}$ & -4.6354451 & 5.9841412 & -1.1109417 \\
\hline 0 & -4.4545737 & 3.2863118 & -0.8607054 \\
\hline $\mathrm{H}$ & -1.9757450 & 4.7584802 & 0.4808050 \\
\hline C & 0.8085027 & 0.8372173 & 1.6198755 \\
\hline C & -0.7568387 & 0.8376063 & 1.6476917 \\
\hline $\mathrm{H}$ & 1.1819309 & 1.0724072 & 2.6273525 \\
\hline $\mathrm{C}$ & 1.4324640 & -0.5045847 & 1.2091291 \\
\hline $\mathrm{H}$ & -1.0939644 & 1.0733801 & 2.6676645 \\
\hline $\mathrm{C}$ & -1.3959724 & -0.5037837 & 1.2598464 \\
\hline $\mathrm{H}$ & -2.4798067 & -0.3915104 & 1.1121639 \\
\hline $\mathrm{H}$ & -1.2771663 & -1.1837074 & 2.1188213 \\
\hline $\mathrm{H}$ & 1.3427703 & -1.1845945 & 2.0716820 \\
\hline $\mathrm{H}$ & 2.5106647 & -0.3937877 & 1.0236001 \\
\hline C & -0.7759425 & -1.1650289 & 0.0104056 \\
\hline $\mathrm{H}$ & -1.1680398 & -0.6724047 & -0.8913730 \\
\hline C & -1.1756514 & -2.6286980 & -0.0925025 \\
\hline C & 0.7683501 & -1.1632950 & -0.0190243 \\
\hline C & 1.1671458 & -2.6253494 & -0.1441757 \\
\hline $\mathrm{H}$ & 1.1265883 & -0.6663658 & -0.9321849 \\
\hline O & 2.2993583 & -3.0836146 & -0.2056232 \\
\hline 0 & -2.3074931 & -3.0914968 & -0.0903104 \\
\hline $\mathrm{N}$ & -0.0043587 & -3.4054624 & -0.1813035 \\
\hline C & -0.0047297 & -4.8356423 & -0.3082832 \\
\hline C & -0.5466397 & -5.4259317 & -1.4556588 \\
\hline C & -0.5485404 & -6.8192711 & -1.5731017 \\
\hline C & -0.0051642 & -7.6125208 & -0.5556611 \\
\hline $\mathrm{C}$ & 0.5394661 & -7.0116086 & 0.5854145 \\
\hline $\mathrm{C}$ & 0.5379438 & -5.6194439 & 0.7160691 \\
\hline $\mathrm{H}$ & -0.9575933 & -4.8000340 & -2.2484283 \\
\hline $\mathrm{H}$ & -0.9714984 & -7.2840394 & -2.4650733 \\
\hline $\mathrm{H}$ & -0.0063706 & -8.6994905 & -0.6517549 \\
\hline $\mathrm{H}$ & 0.9633146 & -7.6268685 & 1.3805743 \\
\hline $\mathrm{H}$ & 0.9498824 & -5.1445447 & 1.6071065 \\
\hline
\end{tabular}

\section{Product $5+\mathrm{D}$ (exo)}

Imaginary Frequencies $=0$

Energy (BP86/def-TZVP, level of optimization) $=-1471.8572020717 \mathrm{H}$ Energy (M0 6-2X/def2-TZVP) $=-1471.196674614 \mathrm{H}$

$\begin{array}{llll}\text { C } & 1.0988805 & 2.0704379 & -0.1314024\end{array}$

$\begin{array}{llll}\text { C } & 0.5493757 & 2.3445239 & 1.2659862\end{array}$ 


\begin{tabular}{|c|c|c|c|}
\hline C & -0.7825546 & 2.2542387 & 1.1642531 \\
\hline $\mathrm{C}$ & -1.0697960 & 1.9322689 & -0.3001937 \\
\hline 0 & 0.0322731 & 2.6056578 & -0.9769651 \\
\hline $\mathrm{H}$ & 1.1596537 & 2.4653001 & 2.1596018 \\
\hline $\mathrm{H}$ & -1.5308379 & 2.2822828 & 1.9542522 \\
\hline C & 2.4430383 & 2.6780130 & -0.4913887 \\
\hline $\mathrm{H}$ & 2.6663965 & 2.4950546 & -1.5522315 \\
\hline $\mathrm{H}$ & 3.2307707 & 2.2068570 & 0.1118212 \\
\hline C & -2.4001287 & 2.4060778 & -0.8779237 \\
\hline $\mathrm{H}$ & -2.4803371 & 3.4919876 & -0.7369296 \\
\hline $\mathrm{H}$ & -2.4418531 & 2.1947061 & -1.9558658 \\
\hline $\mathrm{N}$ & 2.4939574 & 4.1096245 & -0.2253727 \\
\hline $\mathrm{N}$ & -3.5456597 & 1.7903141 & -0.2214471 \\
\hline C & 3.3279440 & 4.6814261 & 0.6837628 \\
\hline C & -4.3465380 & 2.4421802 & 0.6684940 \\
\hline C & -5.5228174 & 1.6467378 & 1.1961435 \\
\hline $\mathrm{H}$ & -6.4510543 & 2.1811970 & 0.9501217 \\
\hline $\mathrm{H}$ & -5.5771481 & 0.6286150 & 0.7902102 \\
\hline $\mathrm{H}$ & -5.4518775 & 1.5922602 & 2.2912398 \\
\hline 0 & -4.1315834 & 3.6133497 & 1.0280983 \\
\hline $\mathrm{H}$ & -3.8146364 & 0.8512198 & -0.5018085 \\
\hline C & 3.2236928 & 6.1872899 & 0.8143454 \\
\hline $\mathrm{H}$ & 2.4829011 & 6.6319748 & 0.1379202 \\
\hline $\mathrm{H}$ & 2.9570632 & 6.4361490 & 1.8510553 \\
\hline $\mathrm{H}$ & 4.2082218 & 6.6306713 & 0.6099209 \\
\hline 0 & 4.1324462 & 4.0198994 & 1.3676123 \\
\hline $\mathrm{H}$ & 1.8599309 & 4.7095925 & -0.7464840 \\
\hline $\mathrm{C}$ & 0.9147671 & 0.5209551 & -0.3654597 \\
\hline C & -0.6539903 & 0.4190235 & -0.4911423 \\
\hline $\mathrm{H}$ & 1.2714019 & -0.0230544 & 0.5219444 \\
\hline C & 1.6438626 & -0.0145328 & -1.6055521 \\
\hline $\mathrm{H}$ & -1.0696020 & -0.1835271 & 0.3303212 \\
\hline C & -1.0977319 & -0.1832521 & -1.8303136 \\
\hline $\mathrm{H}$ & 1.4770009 & 0.6503526 & -2.4657006 \\
\hline $\mathrm{H}$ & 2.7287338 & -0.0512906 & -1.4299773 \\
\hline $\mathrm{H}$ & -0.8779315 & 0.5103726 & -2.6551827 \\
\hline $\mathrm{H}$ & -2.1829529 & -0.3615493 & -1.8401492 \\
\hline C & -0.3819677 & -1.5159147 & -2.1246406 \\
\hline $\mathrm{H}$ & -0.6750391 & -1.8599715 & -3.1278119 \\
\hline $\mathrm{C}$ & 1.1540757 & -1.4226969 & -1.9970455 \\
\hline $\mathrm{C}$ & 1.5343519 & -2.4763336 & -0.9645506 \\
\hline $\mathrm{H}$ & 1.6477123 & -1.7189405 & -2.9347573 \\
\hline C & -0.7975730 & -2.6152119 & -1.1549467 \\
\hline 0 & -1.9315610 & -3.0078709 & -0.9219871 \\
\hline 0 & 2.6553696 & -2.7447722 & -0.5577467 \\
\hline $\mathrm{N}$ & 0.3603635 & -3.1285705 & -0.5394496 \\
\hline $\mathrm{C}$ & 0.3457207 & -4.2057520 & 0.4097113 \\
\hline $\mathrm{C}$ & 0.7599053 & -3.9739166 & 1.7261443 \\
\hline C & 0.7482283 & -5.0290882 & 2.6435523 \\
\hline C & 0.3169826 & -6.3008816 & 2.2486503 \\
\hline $\mathrm{C}$ & -0.0998427 & -6.5209646 & 0.9304887 \\
\hline $\mathrm{C}$ & -0.0831321 & -5.4744940 & 0.0034447 \\
\hline $\mathrm{H}$ & 1.0848706 & -2.9786545 & 2.0304433 \\
\hline
\end{tabular}




$\begin{array}{lrrr}\mathrm{H} & 1.0725385 & -4.8536203 & 3.6704451 \\ \mathrm{H} & 0.3057700 & -7.1209895 & 2.9683471 \\ \mathrm{H} & -0.4350827 & -7.5113164 & 0.6187299 \\ \mathrm{H} & -0.3956198 & -5.6398528 & -1.0281686\end{array}$

\section{TS $1+E$}

Imaginary Frequencies $=1(-368.20 \mathrm{~cm}-1)$

Energy (BP86/def-TZVP, level of optimization) $=-1262.9404895094 \mathrm{H}$ Energy (M0 6-2X/def2-TZVP) $=-1262.386466187 \mathrm{H}$

C $\quad-0.2207565$

C $\quad 0.7130937$

C $\quad 0.6392696$

C $\quad-2.0088893$

$\mathrm{H} \quad 1.3728096$

C $\quad 0.6011287$

C $\quad 1.3933723$

$-0.2780265 \quad 0.7203683$

$-1.2517584 \quad 1.2618319$

$-1.8057995 \quad 0.3331449$

$-1.3568385$

$-1.0361190$

$0.5520070 \quad 1.6388444$

$-1.7706582$

$-1.7325002$

$\begin{array}{ll}1.2814008 & 0.1332078\end{array}$

1.1285831

0.5734935

$-0.7969946$

$-0.1512304$

1.4853703

1.4904725

1.9820081

$-0.2241673$

$\begin{array}{ll}\mathrm{H} & -0.1512304 \\ \mathrm{H} & 2.3663996\end{array}$

0.2254980

$-0.4482838$

1.3599956

1.0299621

$-2.2140272$

0.4853827

1.7196198

$-2.7220946$

2.4448298

0.5755764

$-2.8926556$

$-1.6029726$

$-0.4658109$

$-0.6714710$

$-1.0597575$

$-0.6537840$

$-1.5556571$

$-2.7054943$

0.7693982

1.7080489

$-3.0976623$

2.0986989

$-2.5569328$

3.0215744

$-1.6344060$

2. 6225778

$-0.1146563$

$-0.1257739$

$-1.1457532$

$-0.3841859-2.4661898$

$-3.2283652$

$-0.9539545$

$-3.3552768$

$-1.2727318 \quad-2.9064046$

$-1.0208369$

2.4169369

$-3.1056864$

0.0415615

$-3.8204460$

2. 4289888

0.8849885

$-2.8649541$

4.0680351

$-0.8109886$

$-1.2237108$

3. 3488177

$-3.6298461$

0.3138663

$-0.4811672$

$-4.2340181$

$-0.1464624$

$-2.8169001$

$-2.5750628$

$-1.1492427$

$-4.3912322$

$-1.7207999$

$-3.5811623$

$-0.2887961$

1.6950454

1.0495662

$-2.4368091$

0.2730785

2.7957145

$-2.3012612$

0.9789932

$-4.2879973$

2.5333498
3.4530763

0.5197168

$-4.6607925$

1.6639928

0.6129022

$-4.8480466$

2.5897840

2.0722674

$-4.3633728$

1. 9804130

2.0826688
0.4312030

0.8855899

2.1606178

2.4455903

3. 5047257

2.7385639

3. 8817370

0.2207791

3.5105972

4.1342821

0.8478159

1.8415703

3. 4771313 


\section{Product $1+\mathbf{E}$}

Imaginary Frequencies $=0$

Energy (BP86/def-TZVP, level of optimization) $=-1262.9889214664 \mathrm{H}$

Energy (M06-2X/def2-TZVP) $=-1262.453497659 \mathrm{H}$

C $\quad 2.2923568 \quad-0.7295198 \quad-0.9737188$

$\begin{array}{llll}\text { C } & 3.6529404 & -0.9376480 & -0.6693956\end{array}$

$\begin{array}{llll}\text { C } & 4.0354802 & -0.5972132 & 0.7559782\end{array}$

$\begin{array}{llll}\text { C } & 0.0367011 & 0.1409183 & -0.0329847\end{array}$

$\begin{array}{llll}\mathrm{H} & 5.1034218 & -0.7531719 & 0.9477789\end{array}$

C $\quad 1.6633534 \quad-1.2760555 \quad 1.3845116$

C $\quad 3.1672281 \quad-1.5488309 \quad 1.6655560$

C $\quad 0.8821025 \quad-2.5484375 \quad 1.0817187$

$\mathrm{H} \quad 1.2272390 \quad-0.8570345 \quad 2.3042388$

$\mathrm{H} \quad 3.4364440 \quad-2.5785780 \quad 1.3973499$

C $\quad 3.5174965 \quad-1.3664660 \quad 3.1397241$

$\begin{array}{llll}0 & 2.8140890 & -0.8285205 & 3.9816829\end{array}$

$\begin{array}{llll}\text { C } & 2.2329024 & 1.0512744 & 0.7428714\end{array}$

C $\quad 3.5921693 \quad 0.8227815 \quad 1.0234480$

C $\quad 4.5322731-1.4401890 \quad-1.6306038$

C $\quad 4.0553359 \quad-1.7336860 \quad-2.9146654$

C $\quad 2.7042249 \quad-1.5346875 \quad-3.2186215$

C $\quad 1.8177837 \quad-1.0388256 \quad-2.2517998$

C $\quad 1.6830912 \quad 2.3208116 \quad 0.9314346$

C $\quad 2.4980058 \quad 3.3621261 \quad 1.3992663$

C $\quad 3.8485484 \quad 3.1336860 \quad 1.6817306$

$\begin{array}{llll}\text { C } & 4.3998398 & 1.8583976 & 1.4961305\end{array}$

$\mathrm{H} \quad 5.5832453 \quad-1.5991617 \quad-1.3790933$

$\mathrm{H} \quad 4.7378861 \quad-2.1183071 \quad-3.6746993$

$\mathrm{H} \quad 2.3309989 \quad-1.7678207 \quad-4.2175575$

$\mathrm{H} \quad 0.7692064 \quad-0.8905230 \quad-2.5008672$

$\mathrm{H} \quad 0.6311389 \quad 2.5191165 \quad 0.7178970$

$\mathrm{H} \quad 2.0709180 \quad 4.3564058 \quad 1.5404765$

$\mathrm{H} \quad 4.4763143 \quad 3.9496339 \quad 2.0439121$

$\mathrm{H} \quad 5.4551573 \quad 1.6751812 \quad 1.7102394$

$\begin{array}{llll}0 & -0.7164214 & 0.0636597 & 1.0891289\end{array}$

$\begin{array}{llll}\mathrm{H} & -1.6266692 & 0.3545034 & 0.8617371\end{array}$

$\begin{array}{llll}0 & 0.1234167 & -2.7024535 & 0.1366778\end{array}$

$\begin{array}{llll}0 & 4.7419905 & -1.8720668 & 3.3976581\end{array}$

$\begin{array}{llll}0 & 1.1111300 & -3.4881618 & 2.0228980\end{array}$

C $\quad 5.2164741 \quad-1.7362793 \quad 4.7695520$

$\mathrm{H} \quad 6.2115447 \quad-2.1891912 \quad 4.7730255$

$\mathrm{H} \quad 5.2716216 \quad-0.6769321 \quad 5.0485817$

$\mathrm{H} \quad 4.5452927 \quad-2.2693724 \quad 5.4539410$

C $\quad 0.3945014 \quad-4.7477551 \quad 1.8615170$

$\mathrm{H} \quad 0.7102499 \quad-5.3648738 \quad 2.7070069$

$\mathrm{H} \quad-0.6877212 \quad-4.5721674 \quad 1.8918215$

$\mathrm{H} \quad 0.6721790 \quad-5.2205280 \quad 0.9114710$

$\begin{array}{llll}0 & -0.4070962 & 0.5463719 & -1.0953556\end{array}$ 


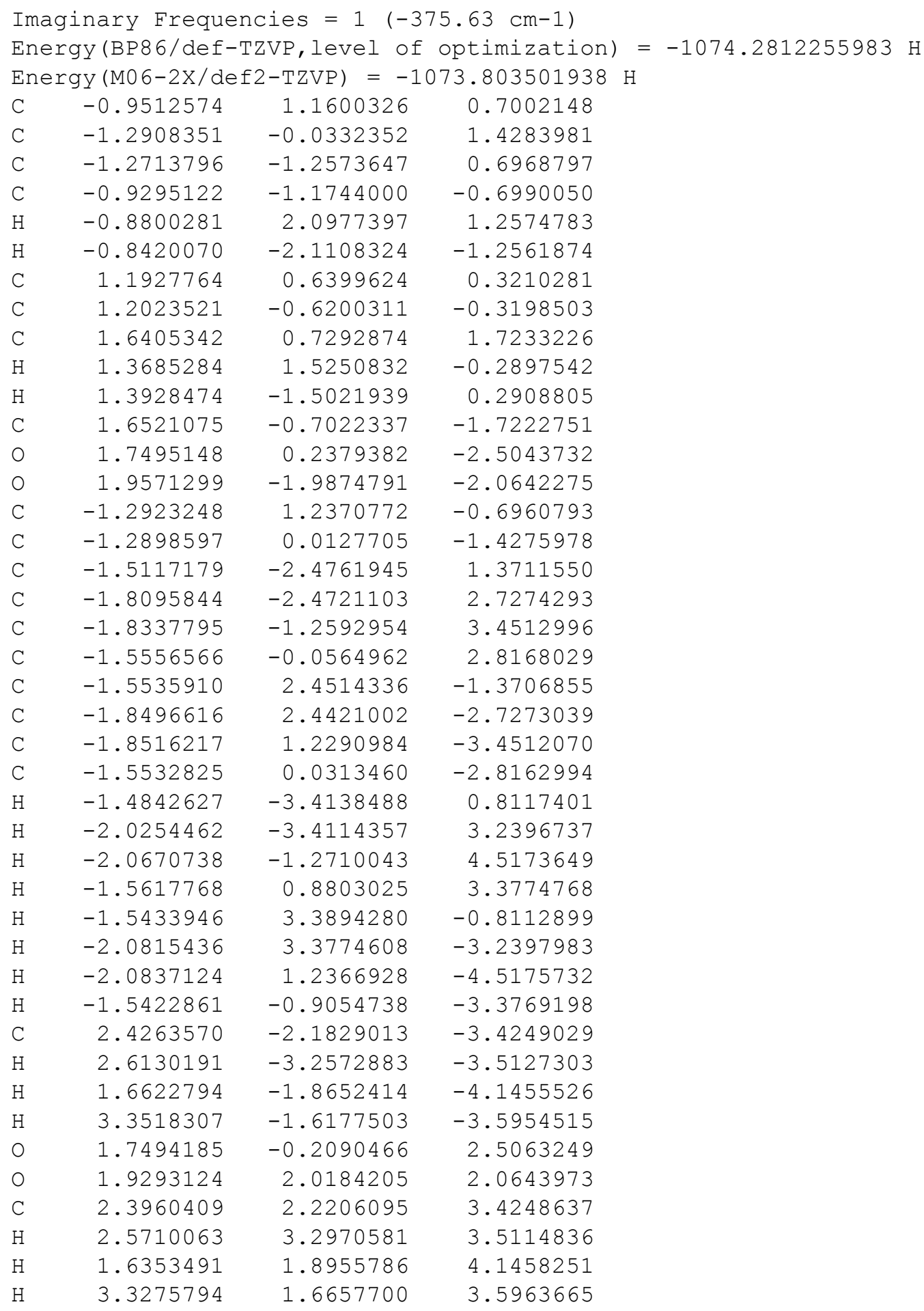

\section{Product $2+\mathbf{E}$}

Imaginary Frequencies $=0$

Energy (BP86/def-TZVP, level of optimization) $=-1074.3301122931 \mathrm{H}$ Energy (M0 6-2X/def2-TZVP) $=-1073.868999888 \mathrm{H}$

$\begin{array}{llll}\text { C } & 0.7584680 & 2.0787421 & -0.8742673 \\ \mathrm{C} & 1.4407252 & 1.4782401 & -2.0892912 \\ \mathrm{C} & 2.8305928 & 1.3251870 & -1.9219645\end{array}$




\begin{tabular}{|c|c|c|c|}
\hline C & 3.3283045 & 1.7953531 & -0.5678214 \\
\hline $\mathrm{H}$ & -0.3198462 & 2.2157032 & -1.0140990 \\
\hline $\mathrm{H}$ & 4.4130641 & 1.6831691 & -0.4581756 \\
\hline C & 1.0423778 & 1.1092108 & 0.3455773 \\
\hline $\mathrm{C}$ & 2.5666722 & 0.9065335 & 0.4989462 \\
\hline C & 0.2969211 & -0.2091801 & 0.1801969 \\
\hline $\mathrm{H}$ & 0.6419081 & 1.5957781 & 1.2469619 \\
\hline $\mathrm{H}$ & 2.8220056 & -0.1420607 & 0.2889328 \\
\hline C & 3.0529282 & 1.2113725 & 1.9100402 \\
\hline O & 2.3964058 & 1.7307218 & 2.8013139 \\
\hline C & 1.4684884 & 3.3768943 & -0.5375540 \\
\hline C & 2.8589561 & 3.2253483 & -0.3724647 \\
\hline C & 3.6075550 & 0.7760328 & -2.9427669 \\
\hline C & 2.9901434 & 0.3865498 & -4.1415488 \\
\hline C & 1.6085914 & 0.5388272 & -4.3082758 \\
\hline C & 0.8259088 & 1.0831957 & -3.2782853 \\
\hline C & 0.8714269 & 4.6280283 & -0.3782730 \\
\hline C & 1.6728216 & 5.7353329 & -0.0597386 \\
\hline C & 3.0548386 & 5.5851011 & 0.1047539 \\
\hline $\mathrm{C}$ & 3.6542064 & 4.3252789 & -0.0474493 \\
\hline $\mathrm{H}$ & 4.6854978 & 0.6554059 & -2.8123919 \\
\hline $\mathrm{H}$ & 3.5924284 & -0.0355528 & -4.9482673 \\
\hline $\mathrm{H}$ & 1.1368034 & 0.2353979 & -5.2447860 \\
\hline $\mathrm{H}$ & -0.2520854 & 1.2030305 & -3.4091477 \\
\hline $\mathrm{H}$ & -0.2073975 & 4.7453197 & -0.5046488 \\
\hline $\mathrm{H}$ & 1.2144471 & 6.7189951 & 0.0589767 \\
\hline $\mathrm{H}$ & 3.6709086 & 6.4518530 & 0.3512768 \\
\hline $\mathrm{H}$ & 4.7327283 & 4.2082348 & 0.0813101 \\
\hline O & 0.8067568 & -1.3064102 & 0.0028158 \\
\hline O & 4.3410162 & 0.8299307 & 2.0608924 \\
\hline 0 & -1.0385023 & -0.0123269 & 0.2501209 \\
\hline C & 4.9349299 & 1.0785424 & 3.3678085 \\
\hline $\mathrm{H}$ & 5.9630280 & 0.7149991 & 3.2877725 \\
\hline $\mathrm{H}$ & 4.9205272 & 2.1517026 & 3.5943636 \\
\hline $\mathrm{H}$ & 4.3881845 & 0.5261807 & 4.1419139 \\
\hline $\mathrm{C}$ & -1.8712275 & -1.1965975 & 0.0850073 \\
\hline $\mathrm{H}$ & -2.9001406 & -0.8348720 & 0.1633786 \\
\hline $\mathrm{H}$ & -1.6950169 & -1.6513096 & -0.8977257 \\
\hline $\mathrm{H}$ & -1.6545815 & -1.9238538 & 0.8768969 \\
\hline
\end{tabular}

\section{TS $3+E$}

Imaginary Frequencies $=1(-348.95 \mathrm{~cm}-1)$

Energy (BP86/def-TZVP, level of optimization) $=-1188.8624105842 \mathrm{H}$

Energy (M06-2X/def2-TZVP) $=-1188.338872701 \mathrm{H}$

$\begin{array}{lrrr}\text { C } & -1.4908769 & -0.0502189 & 0.6178322 \\ \text { C } & -0.7277747 & -1.0359254 & 1.3562616 \\ \text { C } & 0.2784545 & -1.7597154 & 0.6458440 \\ \text { C } & 0.4730697 & -1.4284495 & -0.7451150 \\ \text { C } & -2.4576585 & 0.8618613 & 1.3595949 \\ \text { H } & 1.2473519 & -1.9842586 & -1.2805210 \\ \text { C } & 0.4930006 & 1.3084911 & 0.1358396 \\ \text { C } & 1.4095244 & 0.4137005 & -0.4653747 \\ \text { C } & 0.6249064 & 1.8083108 & 1.4975325\end{array}$




$\begin{array}{rrrr}\mathrm{H} & -0.1021632 & 1.9482192 & -0.5139867 \\ \mathrm{H} & 2.2195352 & 0.0262478 & 0.1521657 \\ \mathrm{C} & 1.7912056 & 0.6782468 & -1.8799655 \\ \mathrm{O} & 1.1576708 & 1.3585998 & -2.6769666 \\ \mathrm{O} & 2.9566814 & 0.0519955 & -2.1923819 \\ \mathrm{C} & -1.6845298 & -0.2771794 & -0.7977118 \\ \mathrm{C} & -0.6907687 & -1.0253469 & -1.4965755 \\ \mathrm{C} & 1.0923880 & -2.6937135 & 1.3219032 \\ \mathrm{C} & 0.8883070 & -2.9526256 & 2.6697257 \\ \mathrm{C} & -0.1126075 & -2.2540757 & 3.3770469 \\ \mathrm{C} & -0.8952977 & -1.3010046 & 2.7408524 \\ \mathrm{C} & -2.8049666 & 0.2051846 & -1.5207303 \\ \mathrm{C} & -2.9443945 & -0.0808724 & -2.8714045 \\ \mathrm{C} & -1.9576344 & -0.8218190 & -3.5557374 \\ \mathrm{C} & -0.8323873 & -1.2703918 & -2.8788988 \\ \mathrm{H} & 1.8674191 & -3.2252801 & 0.7655910 \\ \mathrm{H} & 1.4985468 & -3.6975135 & 3.1831237 \\ \mathrm{H} & -0.2757068 & -2.4657181 & 4.4352021 \\ \mathrm{H} & -1.6641214 & -0.7831604 & 3.3133728 \\ \mathrm{H} & -3.5682151 & 0.7906470 & -1.0101658 \\ \mathrm{H} & -3.8281861 & 0.2680376 & -3.4087410 \\ \mathrm{H} & -2.0804778 & -1.0401917 & -4.6179603 \\ \mathrm{H} & -0.0606728 & -1.8387767 & -3.4021578 \\ \mathrm{O} & -2.5526759 & 2.1860699 & 0.8163364 \\ \mathrm{H} & -1.7284867 & 2.6421371 & 1.1170966 \\ \mathrm{H} & -2.1832600 & 0.9164630 & 2.4220726 \\ \mathrm{H} & -3.4682494 & 0.4237413 & 1.3102111 \\ \mathrm{C} & 3.4346797 & 0.2482016 & -3.5522069 \\ \mathrm{H} & 4.3615506 & -0.3287279 & -3.6158902 \\ \mathrm{H} & 2.6977237 & -0.1239428 & -4.2747604 \\ \mathrm{H} & 3.6296097 & 1.3123085 & -3.7366390 \\ \mathrm{O} & -0.0641121 & 2.7355659 & 1.9584860 \\ \mathrm{O} & 1.5754101 & 1.1847999 & 2.2312770 \\ \mathrm{C} & 1.7318634 & 1.6418455 & 3.6019506 \\ \mathrm{H} & 2.5118401 & 1.0044788 & 4.0279590 \\ \mathrm{H} & 2.0441914 & 2.6936156 & 3.6185364 \\ \mathrm{H} & 0.7924981 & 1.5253511 & 4.1559278\end{array}$

\section{Product $3+\mathbf{E}$}

Imaginary Frequencies $=0$

Energy (BP86/def-TZVP, level of optimization) $=-1188.9079082496 \mathrm{H}$ Energy (M06-2X/def2-TZVP) $=-1188.401615002 \mathrm{H}$

$\begin{array}{lrrr}\text { C } & 1.4672821 & -0.2659376 & 0.1099599 \\ \text { C } & 2.2620995 & -0.8793998 & -1.0463095 \\ \text { C } & 3.6243260 & -1.0819171 & -0.7479987 \\ \text { C } & 4.0131083 & -0.6547003 & 0.6508980 \\ \text { C } & -0.0123843 & -0.0279195 & -0.2365226 \\ \text { H } & 5.0801650 & -0.8068027 & 0.8514246 \\ \text { C } & 1.6387460 & -1.2431972 & 1.3710635 \\ \text { C } & 3.1353043 & -1.5401259 & 1.6214399 \\ \text { C } & 0.7417969 & -2.4653338 & 1.2550014 \\ \text { H } & 1.2430135 & -0.6841318 & 2.2308682 \\ \text { H } & 3.3670790 & -2.5871116 & 1.3909707\end{array}$




$\begin{array}{rrrr}\text { C } & 3.5289939 & -1.2998996 & 3.0746614 \\ \mathrm{O} & 2.8672531 & -0.7000352 & 3.9092976 \\ \mathrm{C} & 2.2184903 & 1.0006734 & 0.5349665 \\ \mathrm{C} & 3.5795121 & 0.7844554 & 0.8268092 \\ \mathrm{C} & 4.4872852 & -1.6371367 & -1.6932464 \\ \mathrm{C} & 3.9922385 & -1.9829774 & -2.9591582 \\ \mathrm{C} & 2.6427074 & -1.7781008 & -3.2633302 \\ \mathrm{C} & 1.7725073 & -1.2298686 & -2.3085719 \\ \mathrm{C} & 1.6803121 & 2.2847716 & 0.6542844 \\ \mathrm{C} & 2.5055655 & 3.3476189 & 1.0516071 \\ \mathrm{C} & 3.8568703 & 3.1288775 & 1.3402201 \\ \mathrm{C} & 4.3982381 & 1.8402293 & 1.2311827 \\ \mathrm{H} & 5.5405671 & -1.7935971 & -1.4494292 \\ \mathrm{H} & 4.6630212 & -2.4089282 & -3.7075718 \\ \mathrm{H} & 2.2596923 & -2.0434437 & -4.2503858 \\ \mathrm{H} & 0.7242585 & -1.0796388 & -2.5693143 \\ \mathrm{H} & 0.6256547 & 2.4691202 & 0.4478639 \\ \mathrm{H} & 2.0860726 & 4.3519015 & 1.1364391 \\ \mathrm{H} & 4.4919522 & 3.9614247 & 1.6484909 \\ \mathrm{H} & 5.4536294 & 1.6635569 & 1.4518918 \\ \mathrm{O} & -0.8244175 & 0.3178670 & 0.8933031 \\ \mathrm{H} & -0.9636259 & -0.5267150 & 1.3789203 \\ \mathrm{H} & -0.4226570 & -0.9217821 & -0.7328881 \\ \mathrm{H} & -0.0867475 & 0.8034240 & -0.9518223 \\ \mathrm{O} & -0.4636684 & -2.4254894 & 1.5057688 \\ \mathrm{O} & 4.7435698 & -1.8346239 & 3.3285659 \\ \mathrm{O} & 1.3652452 & -3.5769143 & 0.8409103 \\ \mathrm{C} & 5.2563469 & -1.6492059 & 4.6800460 \\ \mathrm{H} & 6.2344688 & -2.1377754 & 4.6834335 \\ \mathrm{H} & 5.3564801 & -0.5802561 & 4.9051574 \\ \mathrm{H} & 4.5843498 & -2.1226817 & 5.4063142 \\ \mathrm{C} & 0.5367051 & -4.7697916 & 0.6958500 \\ \mathrm{H} & 1.2205673 & -5.5487476 & 0.3491653 \\ \mathrm{H} & 0.0974237 & -5.0393485 & 1.6637113 \\ \mathrm{H} & -0.2558807 & -4.5895773 & -0.0399046 \\ & & & \end{array}$

\section{TS $4+E$}

Imaginary Frequencies $=1(-383.13 \mathrm{~cm}-1)$

Energy (BP86/def-TZVP, level of optimization) $=-918.6259542323 \mathrm{H}$

Energy (M0 6-2X/def2-TZVP) $=-918.1953886355 \mathrm{H}$

$\begin{array}{lrrr}\text { C } & -1.0197695 & 0.6646214 & -1.2280284 \\ \mathrm{C} & -0.9341767 & -0.5307133 & -1.9561011 \\ \mathrm{C} & 0.2854399 & -1.1637096 & -2.1778076 \\ \mathrm{C} & 1.5117198 & -0.6199648 & -1.7709234 \\ \mathrm{H} & -1.8610853 & -1.0590210 & -2.1899277 \\ \mathrm{H} & 0.2743871 & -2.1888873 & -2.5582833 \\ \mathrm{C} & -0.1584668 & 0.0751019 & 0.6930830 \\ \mathrm{C} & 1.1509700 & -0.3909712 & 0.4533950 \\ \mathrm{C} & -1.1799689 & -0.9158611 & 1.1016073 \\ \mathrm{H} & 1.3622661 & -1.4482890 & 0.6052146 \\ \mathrm{O} & -1.1235487 & -2.1205037 & 0.8760403 \\ \mathrm{C} & -2.3500525 & 1.2550173 & -0.9496430 \\ \mathrm{H} & -0.2035592 & 1.3865253 & -1.2641249\end{array}$




$\begin{array}{rrrr}\mathrm{H} & 1.5985444 & 0.4698142 & -1.7546260 \\ \mathrm{C} & 2.7932574 & -1.3737666 & -1.9655670 \\ \mathrm{H} & 3.5368874 & -1.1305995 & -1.1950344 \\ \mathrm{H} & 2.6281476 & -2.4595069 & -1.9696466 \\ \mathrm{H} & 3.2375972 & -1.0901687 & -2.9350272 \\ \mathrm{O} & -3.4311479 & 0.7050200 & -1.1278137 \\ \mathrm{O} & -2.2391829 & 2.5147807 & -0.4477717 \\ \mathrm{H} & -3.1448316 & 2.8482554 & -0.2681829 \\ \mathrm{H} & -0.2824953 & 1.0877696 & 1.0768475 \\ \mathrm{C} & 2.2494296 & 0.5645908 & 0.6958482 \\ \mathrm{O} & -2.2049397 & -0.3214430 & 1.7622242 \\ \mathrm{C} & -3.2785105 & -1.2038148 & 2.1912176 \\ \mathrm{H} & -3.9996621 & -0.5540499 & 2.6948393 \\ \mathrm{H} & -3.7387844 & -1.6925448 & 1.3237608 \\ \mathrm{H} & -2.8928964 & -1.9619513 & 2.8846575 \\ \mathrm{O} & 2.1565134 & 1.7873564 & 0.6076294 \\ \mathrm{O} & 3.4048149 & -0.0717911 & 1.0249493 \\ \mathrm{C} & 4.5502446 & 0.7892895 & 1.2815788 \\ \mathrm{H} & 5.3719458 & 0.1080625 & 1.5194883 \\ \mathrm{H} & 4.7875397 & 1.3858373 & 0.3917931 \\ \mathrm{H} & 4.3419570 & 1.4541632 & 2.1290096\end{array}$

\section{Product $4+\mathbf{E}$}

Imaginary Frequencies $=0$

Energy (BP86/def-TZVP, level of optimization) $=-918.6823904313 \mathrm{H}$

Energy $(\mathrm{M} 06-2 \mathrm{X} / \mathrm{def2}-\mathrm{TZVP})=-918.2745333291 \mathrm{H}$

$\begin{array}{lrrr}\mathrm{C} & -0.3007833 & -0.0143137 & 0.0154418 \\ \mathrm{C} & 1.0112905 & 0.1299399 & -0.7073638 \\ \mathrm{C} & 1.7139595 & 1.2701290 & -0.7236187 \\ \mathrm{C} & 1.3248368 & 2.5417956 & -0.0142157 \\ \mathrm{H} & 1.3703321 & -0.7465567 & -1.2504083 \\ \mathrm{H} & 2.6592931 & 1.3042450 & -1.2737713 \\ \mathrm{C} & -0.4972115 & 1.0589023 & 1.1067943 \\ \mathrm{C} & -0.1164962 & 2.4659231 & 0.5756773 \\ \mathrm{C} & 0.2551800 & 0.7112570 & 2.3883850 \\ \mathrm{H} & -0.1696245 & 3.1722094 & 1.4193828 \\ \mathrm{O} & 0.9813490 & -0.2596366 & 2.5433070 \\ \mathrm{C} & -0.5546721 & -1.4384866 & 0.4923739 \\ \mathrm{H} & -1.1201644 & 0.1530615 & -0.7116889 \\ \mathrm{H} & 1.3262224 & 3.3524200 & -0.7605593 \\ \mathrm{C} & 2.3805744 & 2.9288020 & 1.0437396 \\ \mathrm{H} & 2.1355704 & 3.8982771 & 1.5009633 \\ \mathrm{H} & 2.4577135 & 2.1759465 & 1.8413779 \\ \mathrm{H} & 3.3714945 & 3.0121243 & 0.5747455 \\ \mathrm{O} & 0.0616814 & -2.4297380 & 0.1329021 \\ \mathrm{O} & -1.6360287 & -1.5048259 & 1.3093848 \\ \mathrm{H} & -1.7783193 & -2.4469306 & 1.5462022 \\ \mathrm{H} & -1.5604940 & 1.0942608 & 1.3851964 \\ \mathrm{C} & -1.1843896 & 2.9570488 & -0.4040291 \\ \mathrm{O} & -0.0203968 & 1.6013320 & 3.3670446 \\ \mathrm{C} & 0.6150100 & 1.3574303 & 4.6550269 \\ \mathrm{H} & 0.2705470 & 2.1691533 & 5.3014848 \\ \mathrm{H} & 0.3003504 & 0.3847159 & 5.0523748\end{array}$




$\begin{array}{lrrr}\mathrm{H} & 1.7068266 & 1.3825353 & 4.5508978 \\ \mathrm{O} & -2.1990231 & 2.3478601 & -0.7158252 \\ \mathrm{O} & -0.8943707 & 4.1931590 & -0.8618796 \\ \mathrm{C} & -1.8633604 & 4.7833012 & -1.7765292 \\ \mathrm{H} & -1.4521377 & 5.7634207 & -2.0330696 \\ \mathrm{H} & -1.9663872 & 4.1597076 & -2.6728071 \\ \mathrm{H} & -2.8355400 & 4.8888094 & -1.2795009\end{array}$

\section{TS $5+E$}

Imaginary Frequencies $=1(-374.47 \mathrm{~cm}-1)$

Energy (BP86/def-TZVP, level of optimization) $=-1259.5777271282 \mathrm{H}$

Energy (M06-2X/def2-TZVP) $=-1258.987461269 \mathrm{H}$

\begin{tabular}{|c|c|c|c|}
\hline $\mathrm{C}$ & -1.0265625 & 0.2660218 & -0.2929149 \\
\hline C & -0.8648292 & -1.1195205 & -0.6392559 \\
\hline $\mathrm{C}$ & 0.4834961 & -1.3697690 & -0.6249750 \\
\hline $\mathrm{C}$ & 1.1278729 & -0.1307452 & -0.3015606 \\
\hline O & 0.2025676 & 0.8846675 & -0.4821769 \\
\hline $\mathrm{H}$ & -1.6754348 & -1.8350513 & -0.7466083 \\
\hline $\mathrm{H}$ & 0.9902480 & -2.3263835 & -0.7183257 \\
\hline $\mathrm{C}$ & -2.2327177 & 1.1392689 & -0.5145136 \\
\hline $\mathrm{H}$ & -2.0603866 & 2.1250995 & -0.06191 \\
\hline $\mathrm{H}$ & -3.1004023 & 0.6785723 & -0.0256344 \\
\hline $\mathrm{C}$ & 2.5658764 & 0.2375688 & -0.5 \\
\hline $\mathrm{H}$ & 2.7779887 & 1.2251692 & -0.11 \\
\hline $\mathrm{H}$ & 3.2123449 & -0.5059364 & -0.06 \\
\hline $\mathrm{N}$ & -2.5625479 & 1.3090095 & -1.92 \\
\hline $\mathrm{N}$ & 2.9073805 & 0.2641157 & -1.96 \\
\hline $\mathrm{C}$ & -3.5842593 & 0.64 & $-2 \cdot 5$ \\
\hline $\mathrm{C}$ & 3.5650386 & -0.7520689 & -2.5 \\
\hline $\mathrm{C}$ & 3.835 & -0.54 & -4.0 \\
\hline $\mathrm{H}$ & 3.330 & -1.34 & -4.6 \\
\hline $\mathrm{H}$ & 3.4937411 & 0.4256166 & -4.4 \\
\hline $\mathrm{H}$ & 4.9146376 & -0.64 & -4.24 \\
\hline O & 3.9241602 & -1.78 & $-1 \cdot 9$ \\
\hline $\mathrm{H}$ & 2.6316596 & 1.077 & -2.5 \\
\hline $\mathrm{C}$ & -3.7788774 & 0.9460227 & -4.00 \\
\hline $\mathrm{H}$ & -3.0825928 & 1.7020814 & -4.3 \\
\hline $\mathrm{H}$ & -3.6492489 & 0.0166832 & -4.5783375 \\
\hline $\mathrm{H}$ & -4.8096 & 1.2920441 & -4.1 \\
\hline O & -4.313 & -0.1578975 & $-1 \cdot 9$ \\
\hline $\mathrm{H}$ & -1.9965259 & 1.9479503 & -2.4775282 \\
\hline $\mathrm{C}$ & 0.6961804 & -0.1755680 & 1.81 \\
\hline $\mathrm{C}$ & -0.6951819 & 0.1267391 & 1.7657848 \\
\hline $\mathrm{C}$ & 1.6176281 & 0.8794453 & 2.2782194 \\
\hline $\mathrm{H}$ & 0.9910157 & -1.1938769 & 2.0 \\
\hline $\mathrm{H}$ & -0.9843238 & 1.1576475 & 1.9 \\
\hline $\mathrm{C}$ & -1.6528657 & -0.9000897 & 2.23 \\
\hline 0 & -1.4168744 & -2.0959127 & 2.3756039 \\
\hline O & -2.8753664 & -0.3520626 & 2.4766335 \\
\hline $\mathrm{C}$ & -3.9031441 & -1.2734470 & 2.9331654 \\
\hline $\mathrm{H}$ & -4.7975966 & -0.6593805 & 3.0 \\
\hline $\mathrm{H}$ & -4.0829218 & -2.0518999 & 2.1 \\
\hline II & -3.6042035 & -1.73 & 3.8 \\
\hline
\end{tabular}




$\begin{array}{llll}\mathrm{O} & 1.3980667 & 2.0883454 & 2.2750175 \\ \mathrm{O} & 2.7899871 & 0.3429977 & 2.7233357 \\ \mathrm{C} & 3.7739795 & 1.2927131 & 3.2149935 \\ \mathrm{H} & 4.6270959 & 0.6845127 & 3.5295530 \\ \mathrm{H} & 4.0702626 & 1.9878329 & 2.4190843 \\ \mathrm{H} & 3.3691452 & 1.8567399 & 4.0648801\end{array}$

\section{Product $5+E$}

Imaginary Frequencies $=0$

Energy (BP86/def-TZVP, level of optimization) $=-1259.6045399387 \mathrm{H}$

Energy (M06-2X/def2-TZVP) $=-1259.030423617 \mathrm{H}$

C $\quad 0.7126847$

C $\quad 2.0298502$

C $\quad 2.6860301$

$1.1075285-2.3068907$

$-0.2938345-2.8827860$

$-0.5344382-2.8779437$

1.7679022

$0.7191536 \quad-2.3112071$

$-0.1024956$

$1.7591409 \quad-2.7367748$

$-0.9717372 \quad-3.1293244$

2.5593024

$-0.6778361$

$-1.4561028$

$-3.1118627$

$-0.5840016$

1.9245029

$-2.6832152$

$-1.5783237$

2.9335120

$-2.2538592$

1.4558466

$-2.2662355$

4.1144027

1.0321827

$-2.7207149$

4.4122587

2.0106740

$-2.3143129$

4.7891529

0.2677971

$-2.3136585$

$-0.8588959$

2.0109657

$-4.1237870$

4.2811803

1.0367104

$-4.1655899$

$-1.9200106$

1.4677029

$-4.7823566$

5.0439128

0.1308685

$-4.8386179$

5.0898634

0.3019465

$-6.3425823$

4.7163822

$-0.6172315$

$-6.8151591$

4.4992664

1.1542863

$-6.7012856$

6.1357531

0.4332542

$-6.6529504$

5.6682704

$-0.7781643$

$-4.2618654$

3.7930763

1.7523928

$-4.6974486$

$-1.9247586$

1.6545359

$-6.2855791$

$-1.0715069$

2.2380094

$-6.6538669$

$-1.9156294$

0.6656277

$-6.7650962$

$-2.8572170$

2.1564461

$-6.5787259$

$-2.8313441$

0.8578883

$-4.1938500$

$-0.1471376$

2.4950964

$-4.6645280$

2.3662975

0.6744516

$-0.7410881$

0.8567821

1.0050745

$-0.7415995$

3.1849555

1.6862121

0.0394017

$-0.3282735$

$-0.3483777$

2.5691617

2.0039382

$-0.3170748$

0.0017149

$-0.0007907$

0.0030597

$-1.1138179$

0.3647914

0.4919448

0.2050516

$-1.2412578$

$-0.3926429$

0.8849673

0.1771245

0.9524393

$-1.3095460$

0.3015061

$-0.6423800$

1.8846814

$-1.8040946$

2.8682909

0.1881238 


$\begin{array}{llll}\mathrm{O} & 4.3012760 & 1.1096464 & 0.5420804 \\ \mathrm{C} & 5.1994816 & 1.9910692 & 1.2777466 \\ \mathrm{H} & 6.0266143 & 1.3517985 & 1.5982443 \\ \mathrm{H} & 5.5615747 & 2.7950577 & 0.6249565 \\ \mathrm{H} & 4.6810281 & 2.4188220 & 2.1445009\end{array}$

